Arc Initiation of High Explosives: Final Report

J. F. McCarrick, C. D. Grant, V. Tang, E. A.

Glascoe, J. M. Zaug, H. Wang

March 22, 2012 
This document was prepared as an account of work sponsored by an agency of the United States government. Neither the United States government nor Lawrence Livermore National Security, LLC, nor any of their employees makes any warranty, expressed or implied, or assumes any legal liability or responsibility for the accuracy, completeness, or usefulness of any information, apparatus, product, or process disclosed, or represents that its use would not infringe privately owned rights. Reference herein to any specific commercial product, process, or service by trade name, trademark, manufacturer, or otherwise does not necessarily constitute or imply its endorsement, recommendation, or favoring by the United States government or Lawrence Livermore National Security, LLC. The views and opinions of authors expressed herein do not necessarily state or reflect those of the United States government or Lawrence Livermore National Security, LLC, and shall not be used for advertising or product endorsement purposes.

This work performed under the auspices of the U.S. Department of Energy by Lawrence Livermore National Laboratory under Contract DE-AC52-07NA27344. 


\title{
Arc Initiation of High Explosives: Final Report
}

\author{
09-ERD-042 \\ November 28, 2011 \\ Sponsoring Directorate: WCI \\ P.I.: James F. McCarrick \\ Team: Christian D. Grant, Vincent Tang, Elizabeth A. Glascoe, Joseph M. Zaug, Han Wang
}

This work performed under the auspices of the U. S. Department of Energy by Lawrence Livermore National Laboratory under Contract DE-AC52-07NA27344 and supported by the Laboratory Directed Research and Development Program (09-ERD-042) at LLNL. 


\section{Table of Contents}

Project Summary _ـ 4

Planned Scope

Executed Scope _ 4

Technical Accomplishments__ 4

Milestones and Publications ___

Project Exit__ 8

1. Introduction 9

1.1. Approach 10

1.2. Layout of this document 11

2. Pulser Design and Operation 12

2.1. Pulser Design__ 12

2.2. Coupon and Holder Design (For Plasma Characterization)__ 15

2.3. Pulser Output Characterization 16

2.4. Revised Holder Designs (For TRIR)__ 18

3. Discharge Characterization with Energetic and Inert Thin Films 22

3.1. Summary of Diagnostics _ 22

3.2. Summary of Results 23

4. Time-Resolved Infrared Spectroscopy of Thin Films 27

4.1. Methodology _ 27

4.2. Results, First Campaign__ 28

4.3. Preliminary Results, Second Campaign: Noise Issues__ 31

4.4. Hardware Failure and Attempted Recovery___ 38

5. Pumped Convective Burn $\quad 40$

5.1. Background 40

5.2. Physical Model 42

5.3. Mathematical Model__ 44

5.4. Various Empirical Choices__ 47

5.4.1. Heat Transfer and Viscous Shear__ 47

5.4.2. External Heating__ 48

5.4.3. Burn Model_ـ 49

5.4.3.1. Hard Threshold Limit_ 50

5.4.3.2. Single-step Arrhenius with Constant Heat Flux _ 51

5.4.3.3. Concluding Remarks on Burn Velocity___ 56

5.5. Some Cylindrical Time-Dependent Threshold Results__ 57

5.6. Burn Rate from Multi-Step Models 61

5.6.1. Eigenvalue Calculations with Existing Multi-Step Models_ 61

5.6.2. Comparison with DAC Data__ 64

5.7. Derivation of a Steady PCB Wave 66

5.7.1. Planar Time-Dependent Simulations___ 67 
5.7.2. Details of the Derivation 72

5.7.1.1. The Heating Region

5.7.1.2. The Burning Region $\quad 76$

5.7.1.3. The Self-Similar Region 77

5.7.3. Discussion of Results__ 80

5.8. Concluding Comments $\quad 81$

5.9. Selected References _ 82

6. Comparative Response of PETN and TATB: A Preliminary Study__ 84

6.1. Description of the Configuration___ 84

6.2. Results 86

6.3. Recommendations for Future Capillary Testing _ 90

Appendix A. Listing of PCB Modeling Tools

Appendix B. MATLAB Code for RLC Fits to Pulser Waveforms 99

Appendix C. MATLAB Code for Fitting Temperature and Density__ 102

Appendix D. Summer Student Report on Numerical Approach to Hydro Modeling. 109

Appendix E. MATLAB Code for Processing TRIR Data__ 133

Appendix F. Drawings of Clamp and Holder Hardware_ 138 


\section{Project Summary}

Arc initiaition of high explosives (HE) can occur when electrical breakdown takes place at an interface or through the bulk of the HE. It can occur in accidental scenarios involving electrostatic discharge (ESD), natural scenarios such as lightning, and engineered scenarios such as spark detonators. The unique combination of short time scale, extremely high temperature, and relatively low pressure results in an initiation regime for which there was no first-principles understanding. The continuing need to understand and improve the safety of HE initiation systems in industrial, conventional munitions, and nuclear explosives environments drives us to acquire such first-principles understanding.

\section{Planned Scope}

This LDRD was funded for three years (FY09-11) at levels of \$340k, \$306k, and \$306k sequentially. At those levels, the planned scope included:

- Design and characterization of a high-power, low-energy "pulser" arc source;

- Thorough characterization of arc discharges across HE surfaces, using thin films for spectroscopic transmission;

- Time-resolved infrared spectroscopy (TRIR) of HE thin films, to study the kinetics at extremely high temperatures. Given the tight resources, only one HE type was planned for study; PETN was chosen because the films are relatively easy to make and it is a common detonator HE.

- Support for modeling was included on a best-effort basis.

\section{Executed Scope}

As ultimately executed, the scope included much less TRIR and more modeling, plus an extremely preliminary comparison of behavior between PETN and TATB. These changes evolved from a) the discovery that using a strong electrical source as the pump in the TRIR pump-probe configuration is extremely difficult to implement in a low-noise fashion, and b) from the unexpected failure of the TRIR femtosecond laser system at the beginning of the main experimental campaign. The repair and re-commissioning of the TRIR system could not be completed within the 36-month bounds of the LDRD and a request to delay the schedule was not granted.

\section{Technical Accomplishments}

Aside from the disheartening failure of the TRIR system, the execution of the remainder of the scope included several substantial technical successes detailed in the remainder of this document (consult the relevant sections for more information on the figures copied below), including:

- Construction and successful operation of a $10 \mathrm{kV}, 400 \mathrm{~mJ}, \sim 4 \mathrm{MW}$ pulser capable of reliable and repeatable operation. The pulser also turned out to be a useful source for the Nuclear Forensics SI, 10-SI-016. 


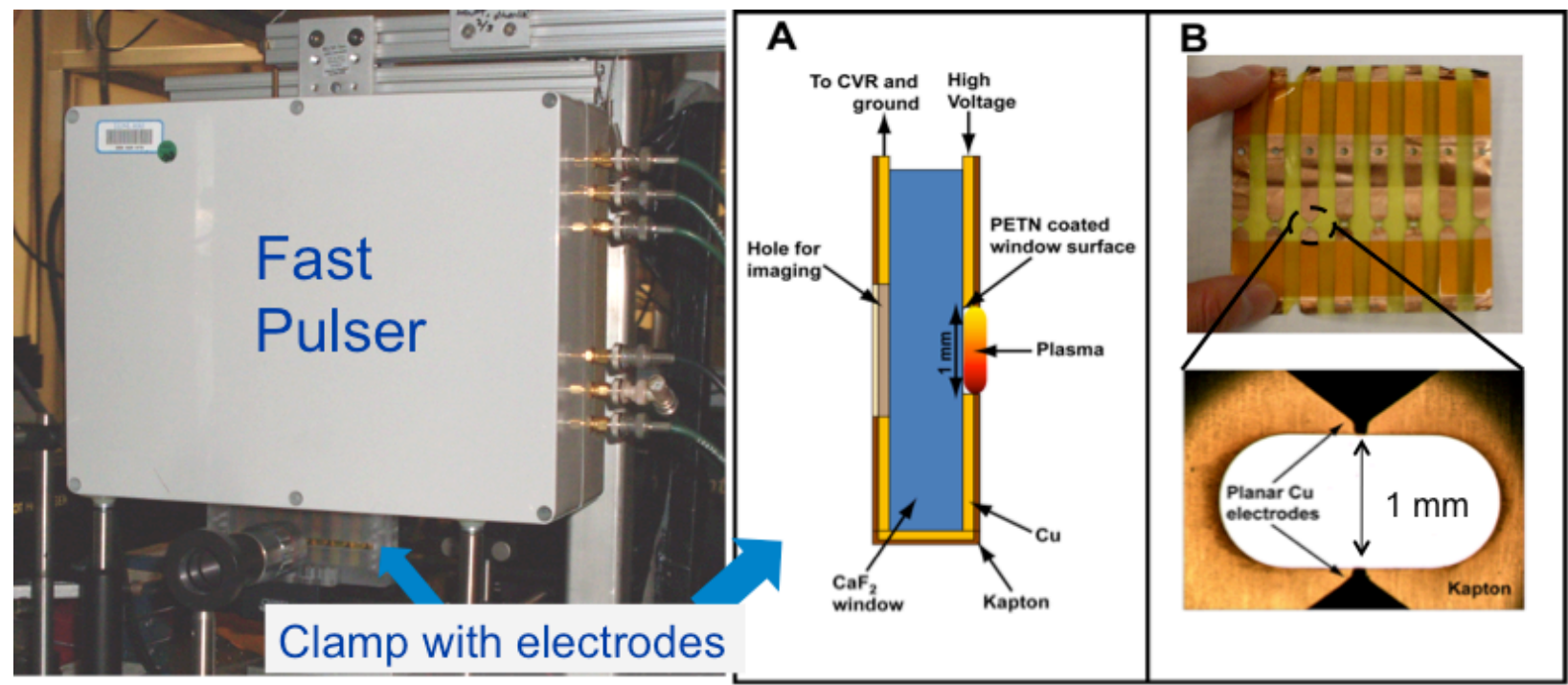

- Thorough spatial and temporal characterization of the temperature and density of typical surface discharges over inert (PMMA) and energetic (PETN) thin films. Temperatures of the discharge are quickly driven to a few $\mathrm{eV}$ during the input pulse, cooling to $1 \mathrm{eV}$ in a few microseconds and persisting at a substantial fraction of an $\mathrm{eV}$ over ten microseconds. Peak electron densities are of order $10^{18} \mathrm{~cm}^{-3}$. In the point-to-point geometry between the pulser electrodes, a discharge is created which undergoes rapid radial expansion at $\sim 2 \mathrm{~mm} / \mu \mathrm{s}$; the properties behind the expansion front are spatially uniform. Even in a confined configuration, the discharge is created in the air above the HE, not through the solid HE itself; convective transport dominates as the top $\sim 300 \mathrm{~nm}$ ablates, and energetic reactions do not propagate to adjacent regions.
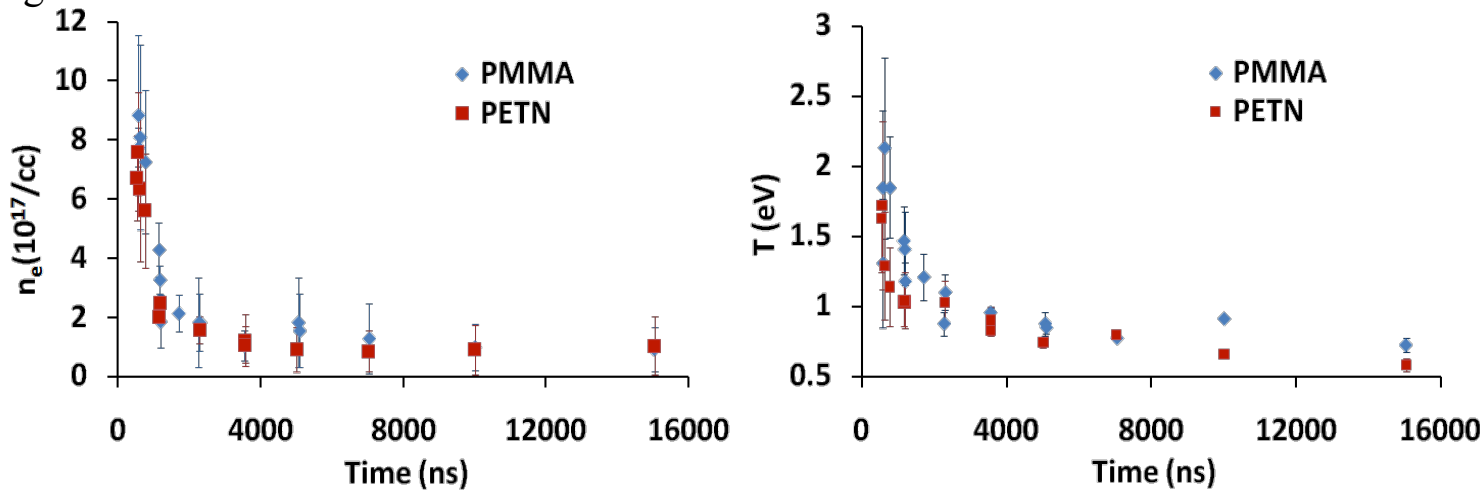

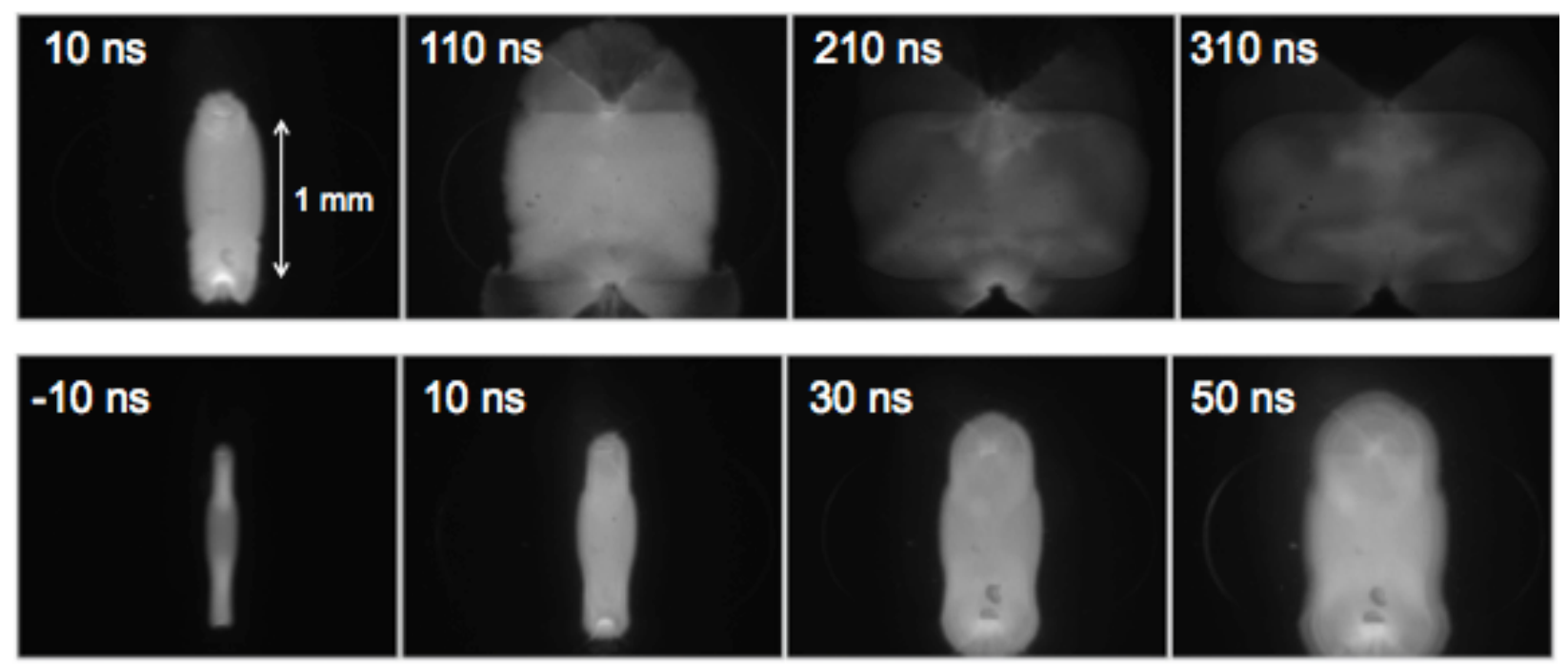

- Development of a model called "pumped convective burn" (PCB), which shows considerable promise at providing first-principles understanding of the arc initiation mechanism in lowdensity HEs. The modeling work includes analytic derivation (using an ideal EOS) of the existence of a quasi-steady PCB wave that can release energy on a fast time scale and short length scale without requiring either shock compression of the solid-phase HE (as in a detonation), or compaction of low-density material (as required in a traditional deflagration-todetonation transition, or DDT). We believe this is the first time such a wave has been identified.

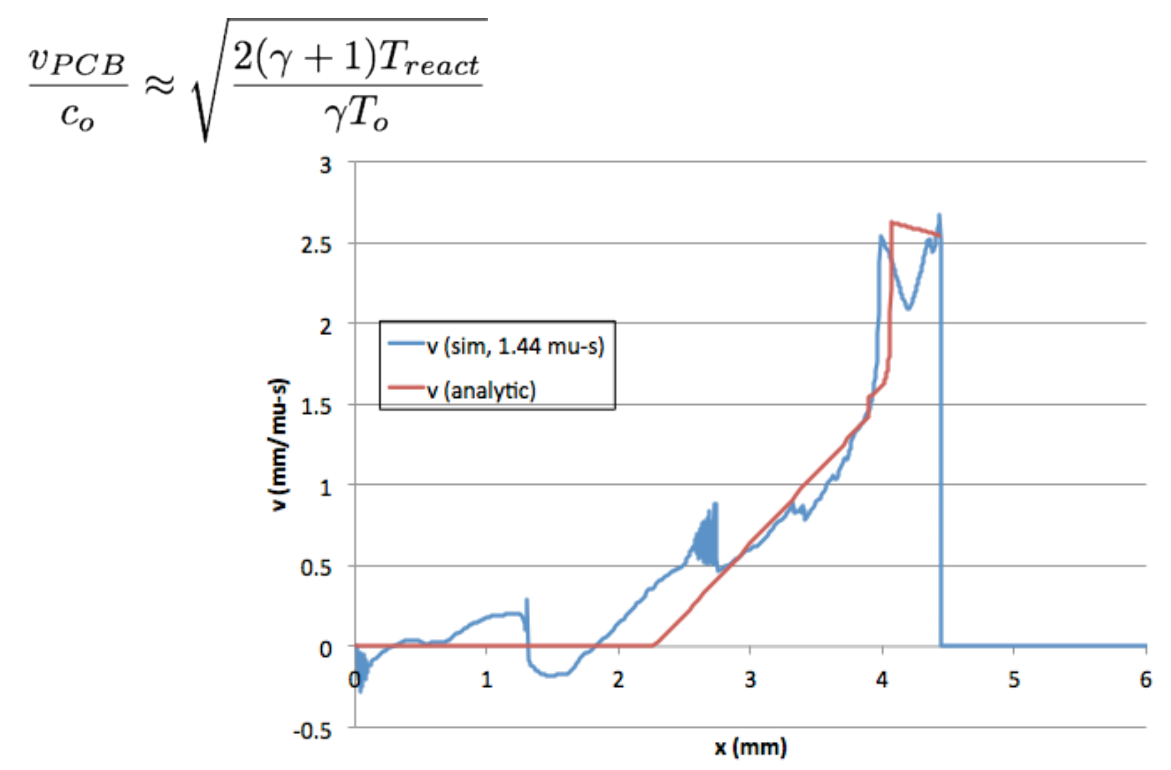

- A first baby-steps study of PCB in a 0-d capillary geometry, which shows that arc-driven PETN can drive expansion of a thin-walled capillary tube at $\sim 1.7 \mathrm{~mm} / \mu$ s in a geometry that precludes any type of run-up to a propagating wave. In the same geometry given the same input arc stimulus, TATB behavior is indistinguishable from that of an inert powder. 

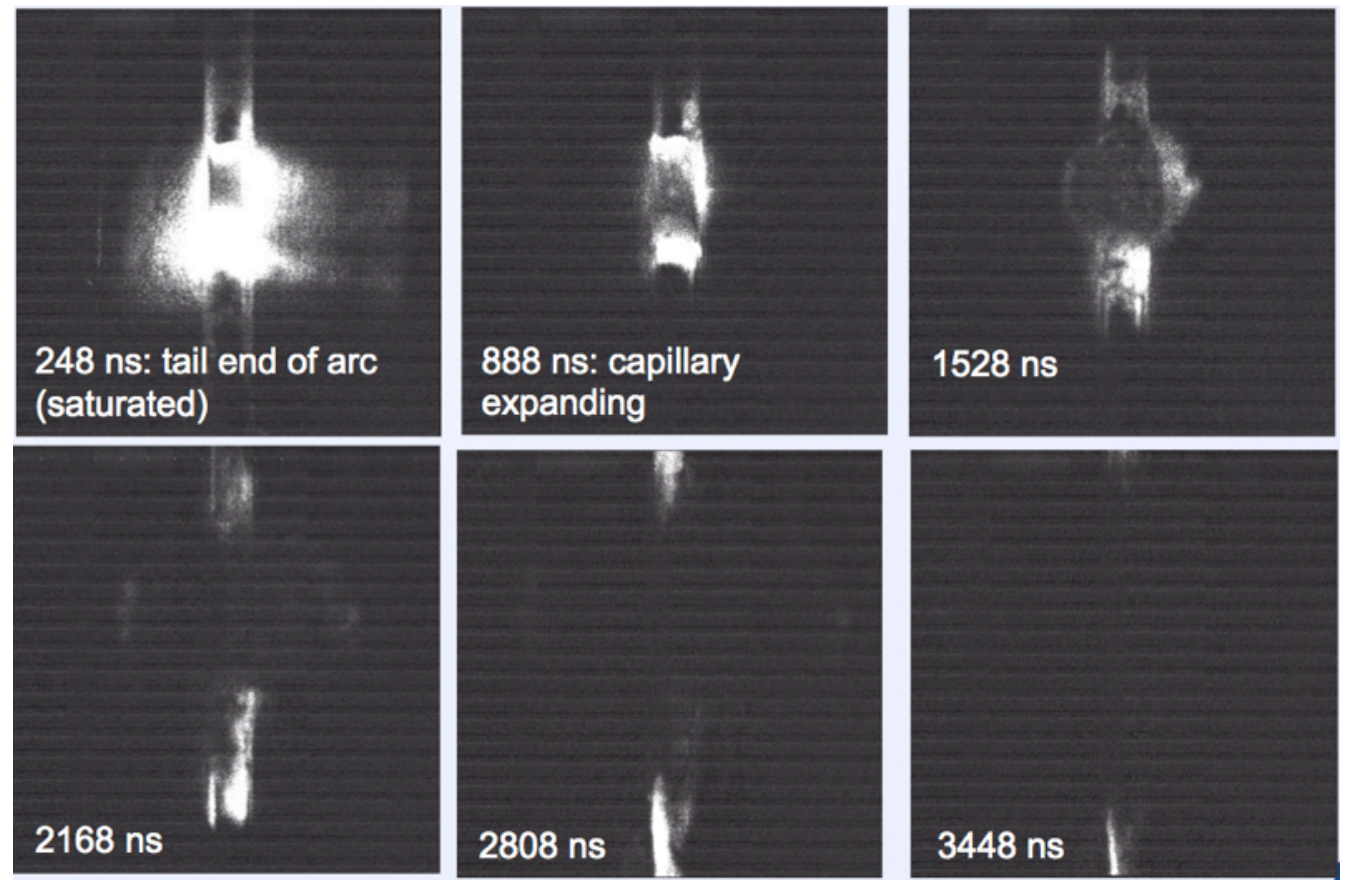

Finally, the preliminary TRIR results that were obtained before the system failure show that an arc-driven pump-probe experiment can be performed, although considerable effort must be made to overcome electrical noise. Simultaneous temperature measurements were also obtained. Some suggestions for how the experiment could be executed successfully in the future are provided in this document.

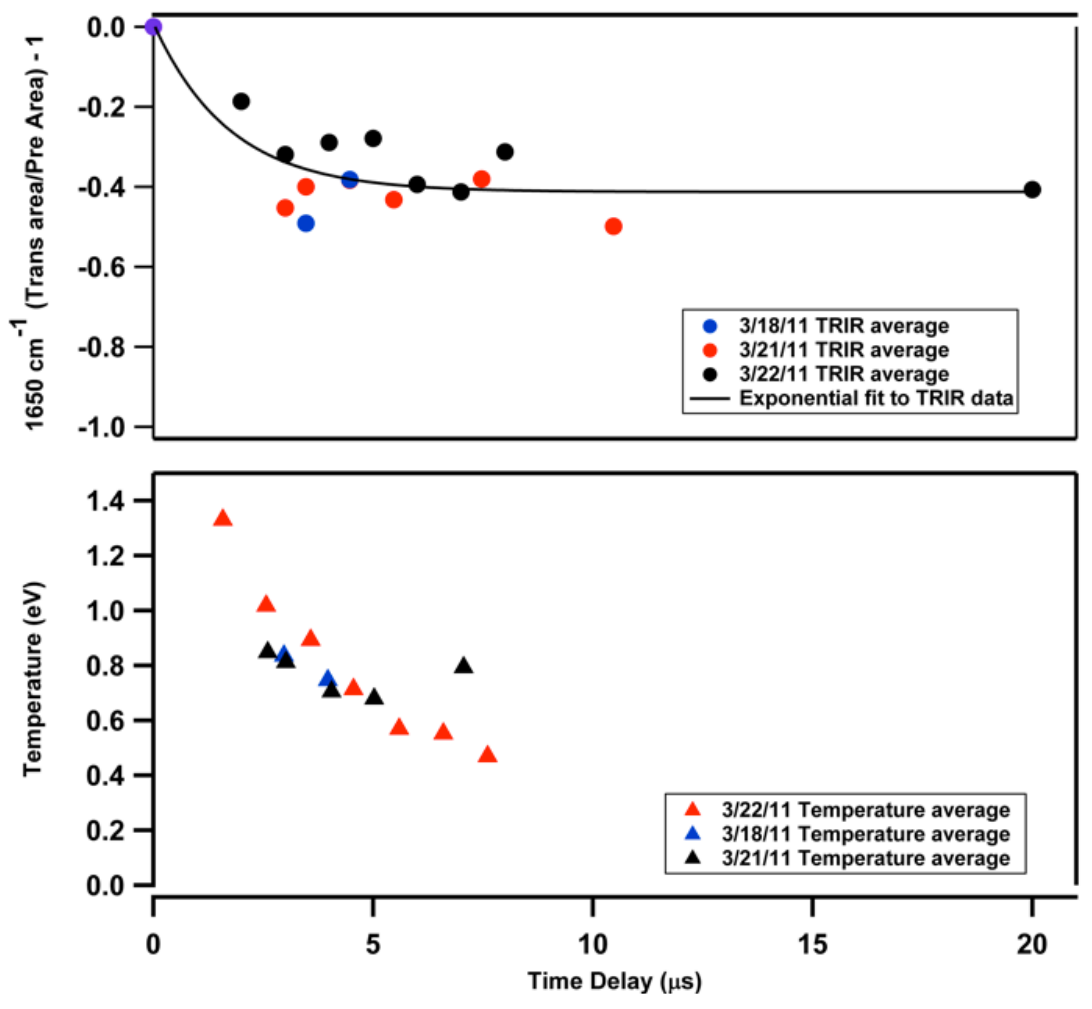


Milestones and Publications

At the beginning of the second year of this LDRD, Chris Grant was successfully converted from post-doctoral appointment to full staff. U.C. Berkeley summer student Han Wang created a modeling tool well above the expectations for a sophomore undergraduate; his work won Best Poster for that year. Mr. Wang returned subsequent years to help with other projects and has applied for a one-year Post College Appointment at LLNL prior to beginning his graduate studies.

The paper "Towards the Understanding of PETN Initiation by a Fast, High-Power Arc Source," by Chris Grant, Vincent Tang, Elizabeth Glascoe, and James McCarrick was published in the peer-reviewed venue of the $14^{\text {th }}$ International Symposium on Detonation, April 2010, pp. 543551. This is also available through IMS as LLNL-CONF-425208.

The paper "Spatiotemporal Temperature and Density Characterization of High Power Atmospheric Flashover Discharges Over Inert PMMA and Energetic PETN Dielectric Surfaces," by Vincent Tang, Chris Grant, James McCarrick, Elizabeth Glascoe, and Han Wang has been published by the Journal of Applied Physics (J. Appl. Phys. 111, 053303 2012). It is also available through IMS as LLNL-JRNL-505792.

Additional papers on the pulser hardware and on the pumped convective burn model are forthcoming; in the meantime, the details are preserved in this report.

\section{Project Exit}

The original exit plan included transfer of the model and techniques developed by this LDRD to both the Nuclear Safety R\&D (NSR\&D) portfolio in WCI/NWE, and the Joint Munitions Program (JMP) TCG-X activities. When the study of TATB was dropped from the original scope, much of the appeal to JMP was lost. However, we have successfully transferred to programmatic NSR\&D funding in FY12, where the follow-on step is generalization from ESDscale arcs to the more general and timely topic of prompt thermal initiation. 


\section{Introduction}

Initiation of high explosives (HE) is the process of transitioning a macroscopic sample of HE from a completely quiescent state to one containing a self-sustained, propagating release of chemical energy. Arc initiation is driven by an electrical discharge across the surface or through the volume of the HE. It can occur in accident scenarios involving electrostatic discharge (ESD) or lightning, and also has had engineered applications such as spark detonators.

Experiments have found that at least one conventional high explosive (pure, low-density pentaerythritol tetranitrate, or PETN) can be arc-initiated with fairly low input energies, as low as a fraction of a $\mathrm{mJ}$ per mil of arc length in a point-to-point discharge geometry. PETN is a common detonator $\mathrm{HE}$ and thus its susceptibility to arc initiation is of interest to the safety of operations in a number of settings, including industry, conventional munitions with the DoD, and plant operations within the NNSA complex.

No predictive capability for arc initiation of HE existed prior to this work. The underlying physics was not known and existing simulation tools could not reproduce the observed behavior. Our ability to understand the thresholds is crucial to the analysis of the safety of existing weapon systems and handling operations. Our ability to predict or engineer them is critical to our capability to design inherently safer initiation systems.

There is presently a significant gap between our understanding of HE initiation at the microscopic level, and the macroscopic level required for real-world applications (note that understanding detonation and understanding initiation are not the same thing!). Predicting the thermal explosion of HE that is heated gradually ("cookoff") is an area that has had success, but the underlying models are often highly empirical with many knobs. By far the largest body of data that exists is for shock initiation, caused by mechanical impacts from supersonic (relative to the HE sound speed) projectiles. The empirical descriptions of shocked HE reaction rates and equations of state are described almost exclusively in terms of pressure; explicit temperature dependence, if present at all, is ad-hoc and calibrated to narrow ranges of data.

In an arc, however, the pressure is too low to drive a significant shock, but the temperature is quite high, corresponding to thermal energies of the same order as the chemical bond strengths $(\sim \mathrm{eV})$. Relative to such levels, existing thermal models such as multi-step Arrhenius kinetics models are calibrated at fairly low temperatures and it is not obvious how well they scale to the arc regime. Also, unlike in a cookoff experiment that can take place over a period of many hours, the arc electrical pulse length is tens of nanoseconds. Thus, in contrast to other modes, arc initiation must rely on fast thermal effects followed by a separate process that leads to propagation in the absence of an initial shock wave.

Thus, the goals of this LDRD are to develop basic understanding of:

- the energy transport mechanism from the arc plasma into the HE;

- the local chemical kinetics driven by very high temperatures; and

- the process by which a locally coupled volume of HE that is driven at a high reaction rate evolves into a propagating reaction with a lower rate. 


\subsection{Approach}

The project plan for this LDRD evolved from the proposal stages according to actual allocated funding and, of course, lessons learned as the work proceeded. Once the actual funding levels were established ( $\$ 340 \mathrm{k}$ in year $1 ; \$ 306 \mathrm{k}$ in subsequent years), the following tasks were planned:

- Design, fabrication, and characterization of a low-energy, high-power pulser to serve as a repeatable arc source for the experiments.

- Thorough spatial and temporal characterization of the temperature, density, and dynamics of discharges over an HE surface, using emission spectroscopy and gated imaging.

- Time-resolved infrared spectroscopy, to infer high-temperature kinetics of arc-driven HE by observing the characteristic decay times of vibrational signatures of unreacted material, and the growth times of the signatures of products. Given PETN as a relevant HE from a historical perspective and TATB (triamino trinitrobenzene) from a contemporary one, resource and schedule constraints forced us to choose just one; we chose PETN because it is far easier to manufacture into the thin films necessary for IR transmission.

- Modeling was to be pursued on a best-effort basis.

The distribution of work as actually executed was somewhat different. When we reached our first serious attempt at TRIR testing, we found that performing a TRIR "pump-probe" experiment with an arc as a probe is not a simple task for a number of reasons, with two especially dominant.

The first major obstacle was throughput. For the arc characterization work, the brightness of the source meant that there was a good correspondence between a single arc pulse and a useful data point. While the holder and coupon setup (described later) had drawbacks, the necessary data was obtainable. However, for TRIR experiments, many more shots are required to obtain a single data point. The signal into the detector is not the strong output from the arc, but the transmitted spectral density from the laser source. Averaging over a number of shots at the same point in time are typically required. On top of that, the precise timing of the probe light relative to the arc cannot be controlled precisely because of the way the pulser and the laser system are triggered; so dialing in the same time at which to take data does not necessarily mean the data is acquired at the desired time, and even more shots are required. The original coupon/holder system was not adequate to achieve anywhere near the required testing throughput, and had to go through a major re-design in the middle of the project, introducing a delay.

The second major obstacle was electrical noise. The pulser is a fast, high-current electrical device and produces considerable electromagnetic radiation. The IR detector array is a sensitive piece of equipment whose electronics, and particularly the grounding scheme, were not accessible to us. The pulser could be modified and wrapped in a number of ways, but there is ultimately no way to deal with the exposed portion of the circuit that is required to drive current along the HE film surface and "pump" the experiment. Considerable time and effort was invested to reduce the noise levels, although we were never successful in getting those levels low enough that data could be taken at "early" times when any substantial arc current was still flowing. 
The time invested in overcoming these obstacles pushed the main TRIR experimental campaign into the third year, at which point a radically different and much more serious obstacle was encountered: failure of the femtosecond laser system that provides the broadband IR probe pulse. This failure ultimately proved fatal to the experiment since it was not possible to extend the LDRD beyond 36 months (we did make a request) while we waited for repairs. More detail on the failure is given in Section 4.

Thus, relative to the planned scope, the executed scope did not include more than the preliminary round of TRIR experiments, where the throughput and noise issues were first identified. However, the down-time during the coupon/holder redesign period through the wait for attempted TRIR repair was used to boost other areas. Significant progress was made in modeling, and at the end of the project, a brief attempt was made to take some data comparing the response of PETN and TATB to arc insults.

\subsection{Layout of this document}

The remainder of this document provides specific details on all the activities performed during the Arc Initiation LDRD, by either direct description or by pointing to other existing documents that were produced along the way.

Section 2 discusses the design and characterization of the pulser, which was the workhorse arc source for the project. Section 3 provides just a brief summary of the work done to study surface discharges over thin films, then points the interested reader to the more extensive descriptions that have been (or soon will be) published in the peer-reviewed literature. Section 4 describes the TRIR campaign to the extent we were able to take it, including a description of the hardware failure that eventually killed it. Section 5 describes the Pumped Convective Burn model, which may prove to be a solid first-principles foundation for the response of HEs to arc initiation and other fast thermal insults; the steady PCB wave derived from the model appears to be a newly identified phenomenon. Finally, a set of appendices provides detail on software tools and hardware design. 


\section{Pulser Design and Operation}

Even under ideal conditions, the signal-to-noise level of TRIR data is not ideal and we were advised from prior efforts that the data from many, many shots would have to be averaged to produce a usable result. In addition, the characterization of the temperature and other properties of the discharges used to pump the HE films was not a simple measurement and, from the beginning, we had planned a separate measurement campaign to characterize the arc plasmas.

Thus, with such a large number of shots required to ultimately obtain our desired data, it was key to have a "workhorse" source of the desired arcs. We had extensive prior experience with the sources used in NSR\&D-sponsored threshold measurements and knew that a highly controlled, repeatable arc is obtainable. However, because the NSR\&D experiments use more significant quantities of HE, resulting in a certain amount of consumables per shot along with shot-rate limitations imposed by operational safety, the arc source could have some limitations that were not rate-limiting in the NSR\&D context but were unacceptable for the LDRD, given the much greater throughput required.

The requirements for the new source were as follows:

- Repeatable arc characteristics, including a well-defined path and only a single branch.

- A triggered spark-gap for the switch, to provide a lifetime of thousands of shots.

- An operating voltage of order $10 \mathrm{kV}$.

- The minimum possible source inductance and resistance.

- High reliability.

These requirements were met with high success by the "pulser" (capacitive discharge unit) described in the following sections. It proved to be an integral component in the characterization shots, the TRIR shots, and the capillary shots carried out by this LDRD, and also ended up being copied for use in the Nuclear Forensics SI, 10-SI-016.

\subsection{Pulser Design}

Figure 2.1 summarizes the arc pulser and the first load geometry (described in more detail in Section 2.2). The arc pulser consists primarily of a high voltage fast capacitor switched by a triggered spark gap. Currently, a fast low inductance $100 \mathrm{kV}$ General Atomics 35436M capacitor with $8 \mathrm{nF}$ is used along with a Perkin-Elmer GP-20B spark gap switch capable of triggered operation up to $11 \mathrm{kV}$. The $100 \mathrm{kV}$ capacitor was used to ensure robustness of the system to voltage overshoots and when operated at $\sim 10-20 \mathrm{kV}$ has an essentially infinite shot-lifetime. Voltages greater than $11 \mathrm{kV}$ can be achieved via installation of a different switch, up to the limit of flashover breakdown inside the pulser. The spark gap is triggered by a MOSFET-switched TR-180B transformer, driven by a $200 \mathrm{~V} 1 \mu \mathrm{F}$ capacitor; the transformer trigger circuit produces up to $18 \mathrm{kV}$ on a $\mu$ s time-scale to trigger the spark gap. A current viewing resistor (CVR) on the ground return with resistance of $140 \mathrm{~m} \Omega$ is used to measure the current. The components sit on a printed circuit board, shown in Figure 2.2, with most of the connections made on the board itself. The circuit board contains wide conducting planes and allows a direct connection to the load in order to minimize system inductance. 
The pulser was designed to trigger as quickly as possible to minimize jitter. Figure 2.3 shows the planned typical control and timing scheme for pulser operation. By using a very fast, high power MOSFET to drive the trigger and by loading the trigger transformer with the highest operating voltage $(200 \mathrm{~V})$, we expected the pulser to commutate its load in approximately $580 \mathrm{~ns}$. This was bored out by experiments as shown by the typical trace in Figure 2.6. The jitter in the system, as discussed in Section 2.3, at start of operations was characterized to be as low as $+/-13$ ns but settled to typically $+/-30$ to $40 \mathrm{~ns}$ for the shots discussed in this report.

a)

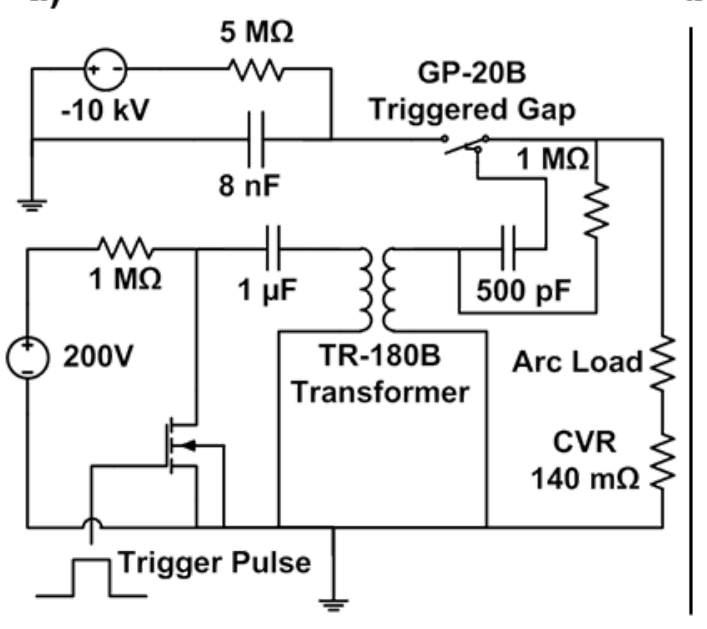

b)

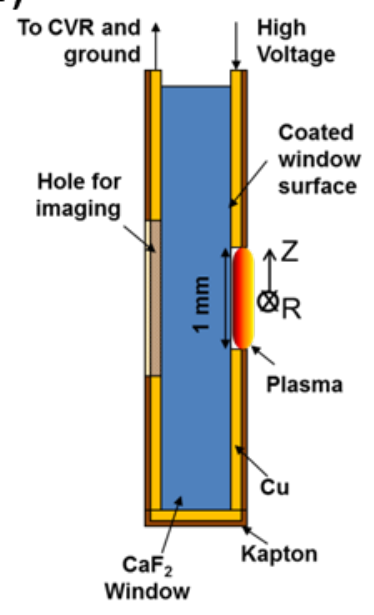

c)

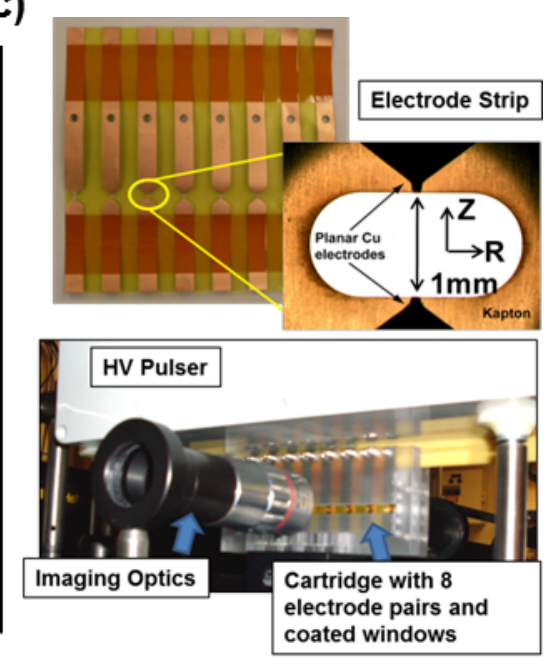

Fig. 2.1: a) Simplified circuit diagram of the high-voltage pulser. b) Diagram of the electrode coupon, which consists of a $17 \mu \mathrm{m}$ thick $\mathrm{Cu}$ strip deposited onto $25 \mu \mathrm{m}$ thick Kapton. The discharge flashes over the $1 \mathrm{~mm}$ long gap and coated film. c-Top) A backlit planar photographic view of the electrodes is shown. The Cu electrodes are $6.3 \mathrm{~mm}$ wide but narrow to $\sim 75 \mu \mathrm{m}$ to form an approximate point-to-point breakdown gap of 1 $\mathrm{mm}$. The white space in the graphic is the transparent $\mathrm{CaF}_{2}$ window material. There is a clear optical path to collect both fast gated images and make spectroscopic measurements. c-bottom) View of the clamp cartridge mated to the $\mathrm{HV}$ pulser. In this setup, there are 8 electrode pairs in the cartridge. After a shot, the cartridge is slid along a so that a fresh pair of electrodes can be mated to the pulser terminals for the next shot. 


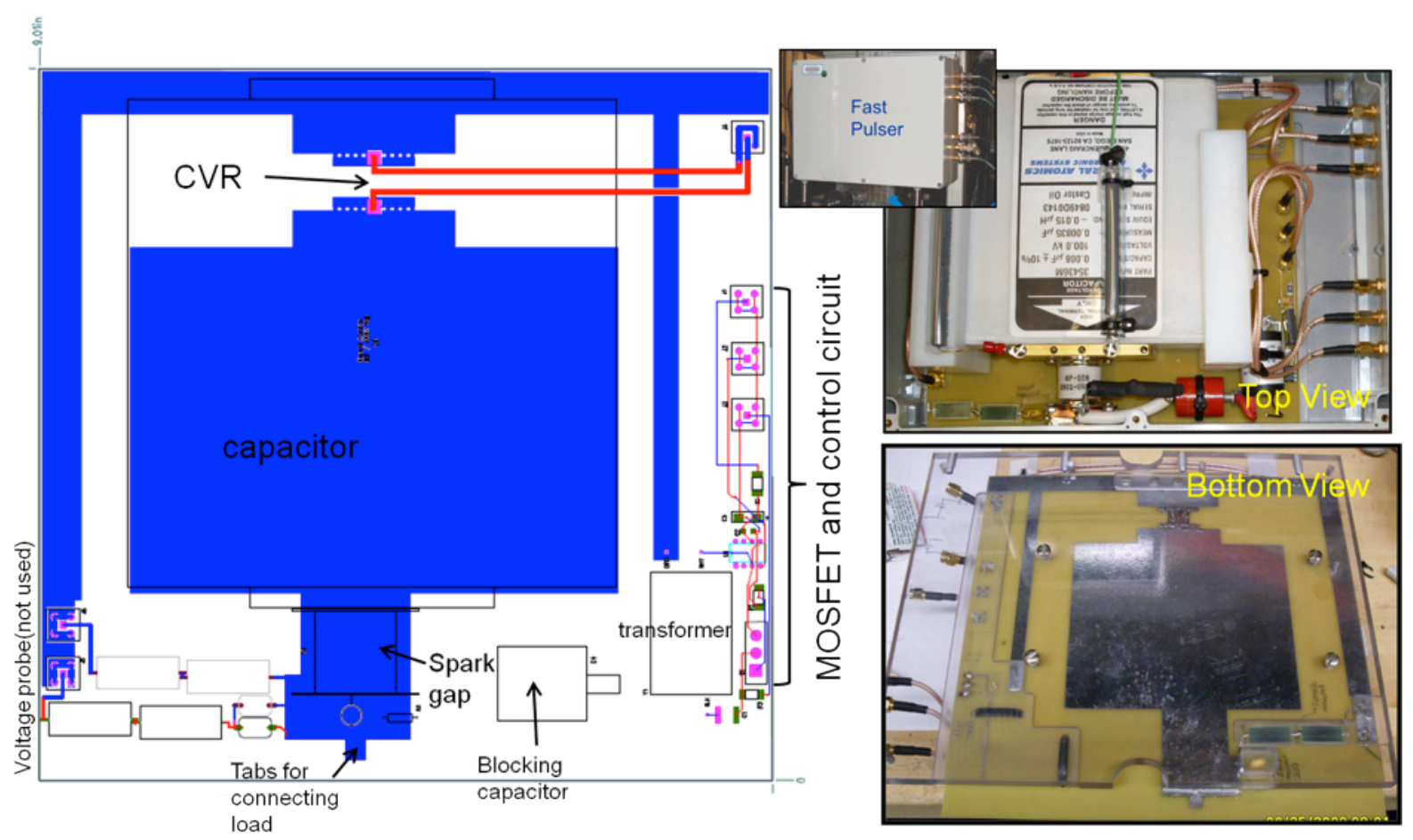

Figure 2.2: Layout of the pulser circuit board along with top and bottom view of the assembled pulser inside its enclosure. The pulser circuit board and its components are supported inside the enclosure by the lexan backplate that is clearly visible in the bottom view.

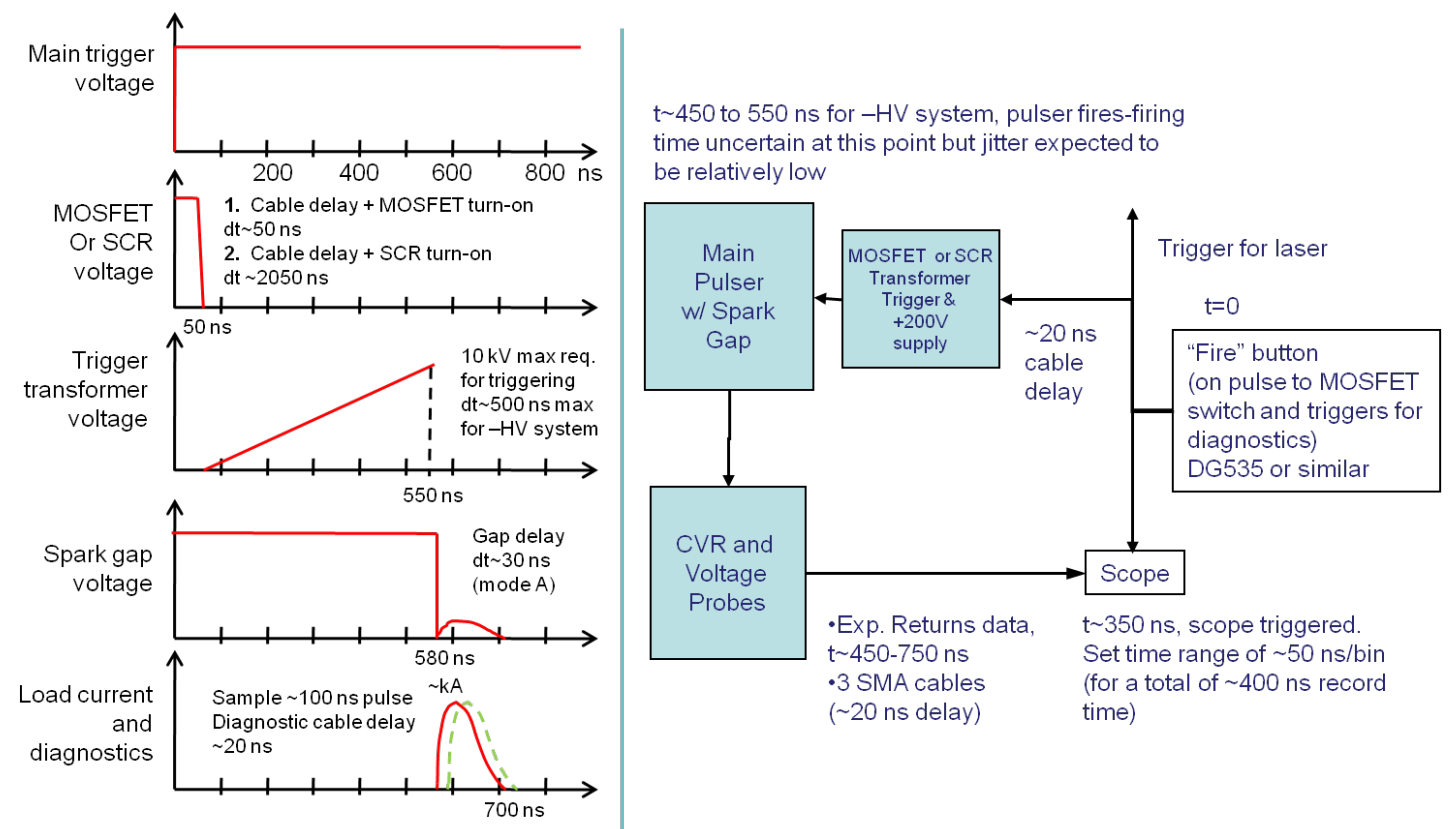

Figure 2.3: Planned timing and control scheme for the arc initiation pulser.

For the majority of the experimental campaign, the pulser was operated at $-10 \mathrm{kV}$ corresponding to a stored energy in the capacitor of $418 \mathrm{~mJ}$. Fitting the CVR waveform data to that of a basic RLC circuit allows us to infer the total time-averaged system inductance, L, and resistance, R, 
for each shot. We note that constant (in time) values of $\mathrm{L}$ and $\mathrm{R}$ are able to match the data well over multiple oscillations. These data along with measurements from baseline shots from a shorted load are used to estimate the power and energy deposited to the load (i.e. electrodes, plasma, and surface). Quantitative specifics are given in Section 2.3.

\subsection{Coupon and Holder Design (For Plasma Characterization)}

The first coupon and holder was designed as a cartridge of 8 electrodes as shown in Figure 2.1(b) and (c). The concept of the design was to allow relatively rapid switching and securing of new electrodes onto the pulser for multiple single-shot experiments. In actual practice a shot rate of 5-6 shots an hour could be achieved, with the majority of the effort going to precise alignment of each new electrode. This allowed us to use this holder for characterizing the plasma, but as discussed, a different design with significantly faster shot rate was ultimately used for the TRIR shots.

The clamp consists of two Lexan pieces with grooves that allow either one or two substrate windows to be clamped to the electrode sheets; this allows the plasma to be either confined or unconfined. Figure 2.1(b) shows the setup for one window. Figure 2.4 provides the schematics. The electrodes on the sheet mated with the pulser tabs using 4-40 set screws along the top of the clamp. For the shots in this report, the substrate window was made from $\mathrm{CaF}_{2}$ and coated with the desired dielectric material for study.

The coupon and electrode for this clamp design consists of a point-to-point setup with a $1 \mathrm{~mm}$ gap, and is made up of $17 \mu \mathrm{m}$ thick $\mathrm{Cu}$ strips with $75 \mu \mathrm{m}$ wide tips deposited onto $25 \mu \mathrm{m}$ thick Kapton backing. The low inductance of the design allows the pulser to deliver multi-MW power to the load. Figure 2.5 shows the drawing for the coupon.

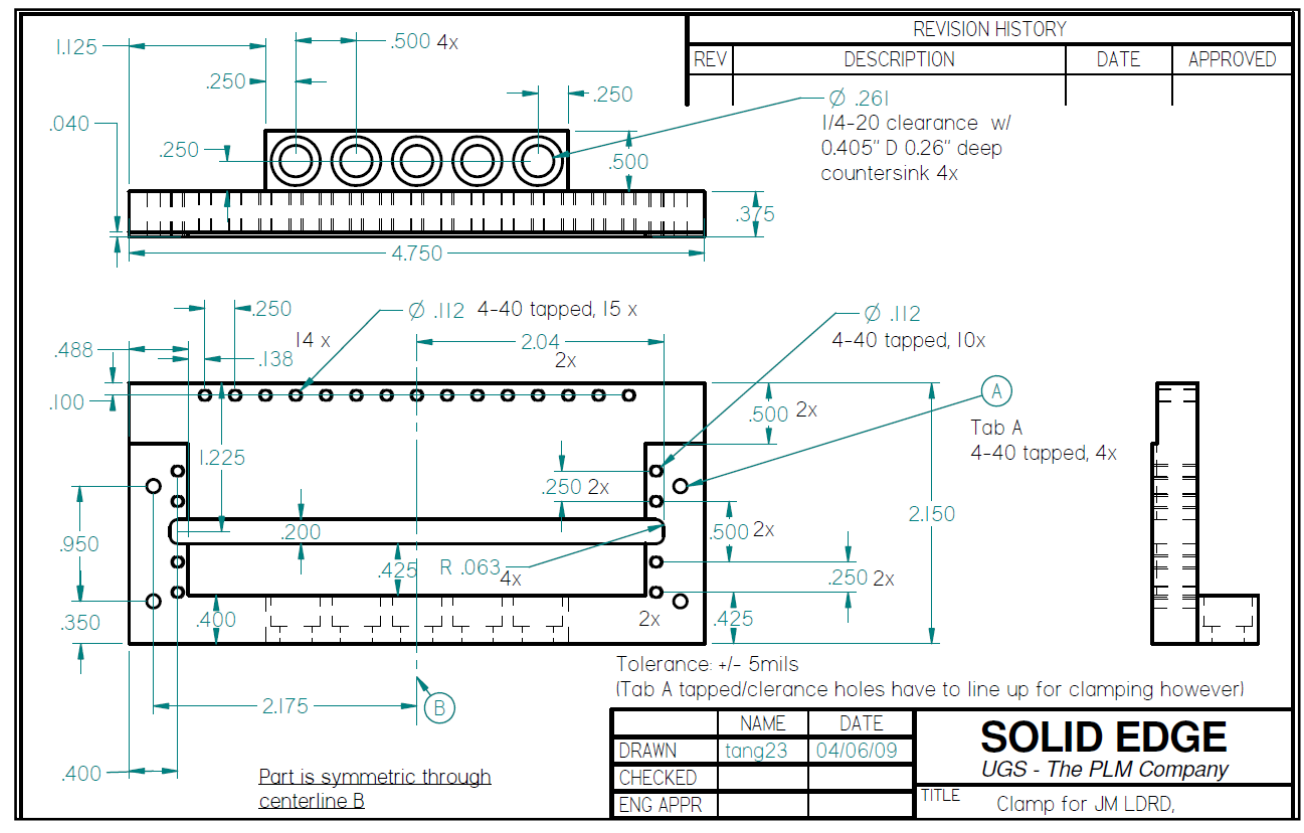

Figure 2.4: Coupon holder design for plasma characterization experiments. 


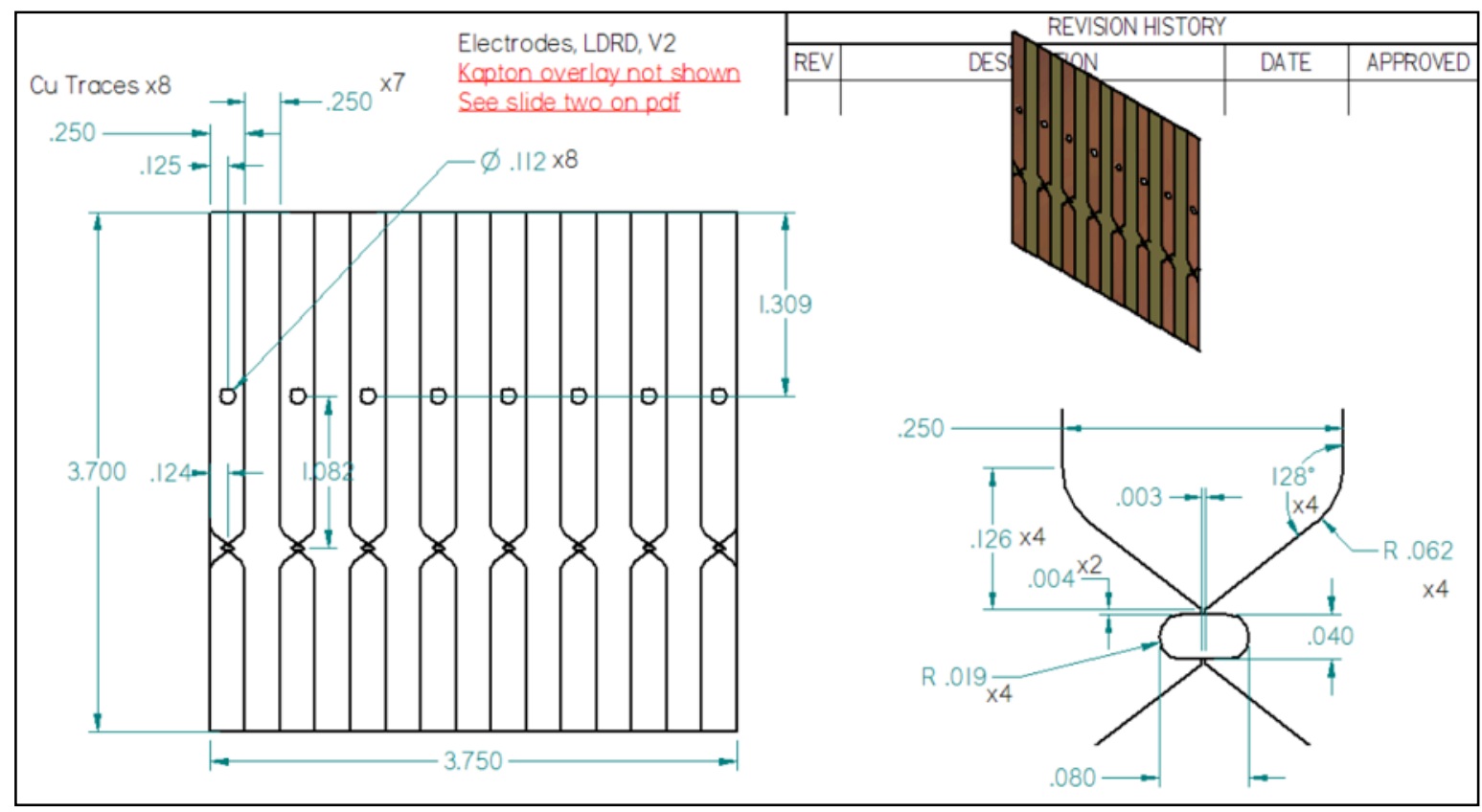

Figure 2.5: 8-electrode coupon design. The kapton overlay seen in Figure 2.1(c) is not shown here but was applied to increase flashover holdoff near the top of the substrate window.

\subsection{Pulser Output Characterization}

Figure 2.6 shows a typical current trace using our pulser along with an RLC fit, assuming constant in time R, L, and C, for a typical unconfined PETN shot using the electrode strip system in Figure 2.1(c). The time delay in current flow is due to switching time inside the pulser. A system inductance of $68.5 \pm 1.8 \mathrm{nH}$ and resistance of $0.74 \pm 0.05 \Omega$ was found for unconfined PMMA experiments over 29 independent experiments. The timing jitter for current flow after trigger is significant at $\pm 44.5 \mathrm{~ns}$ and can make specifying an observation time smaller than this in a single shot difficult; the initial jitter in the system was as low as \pm 13 ns over a series of 30 shots but has grown and settled larger possibly due to erosion in the spark gap. When the pulser terminals were shorted, the resistance was $0.43 \pm 0.03 \Omega$ with inductance of $57.5 \pm 0.1 \mathrm{nH}$ over a sample of 16 experiments. By comparing the plasma measurements with the shorted loads, we estimate the average resistance of the arc load was $0.3 \pm .08 \Omega$ and the average inductance was $11 \pm 1.9 \mathrm{nH}$. We thus estimate the total energy deposited to the plasma, electrodes, and surface typically to be $174 \pm 21 \mathrm{~mJ}$ out of a total available energy of $418 \mathrm{~mJ}$, with peak powers greater than $1 \mathrm{MW}$. For unconfined PETN experiments, a system inductance of $69.1 \pm 0.6 \mathrm{nH}$ and resistance of $0.74 \pm 0.07 \Omega$ was found over 30 replicates. The estimated deposited energy into the PETN loads was thus the same as the energy deposited into the PMMA shots. Figure 2.7 shows a sample series of shorted load data used for the above characterization.

Overall, the electrical shot characteristics was unaffected by the type of film, either PMMA or PETN, for the flashover arc. Approximately $40 \%$ of the pulser energy was delivered to the load for both PMMA and PETN films over a current pulse width of $\sim 500 \mathrm{~ns}$. 


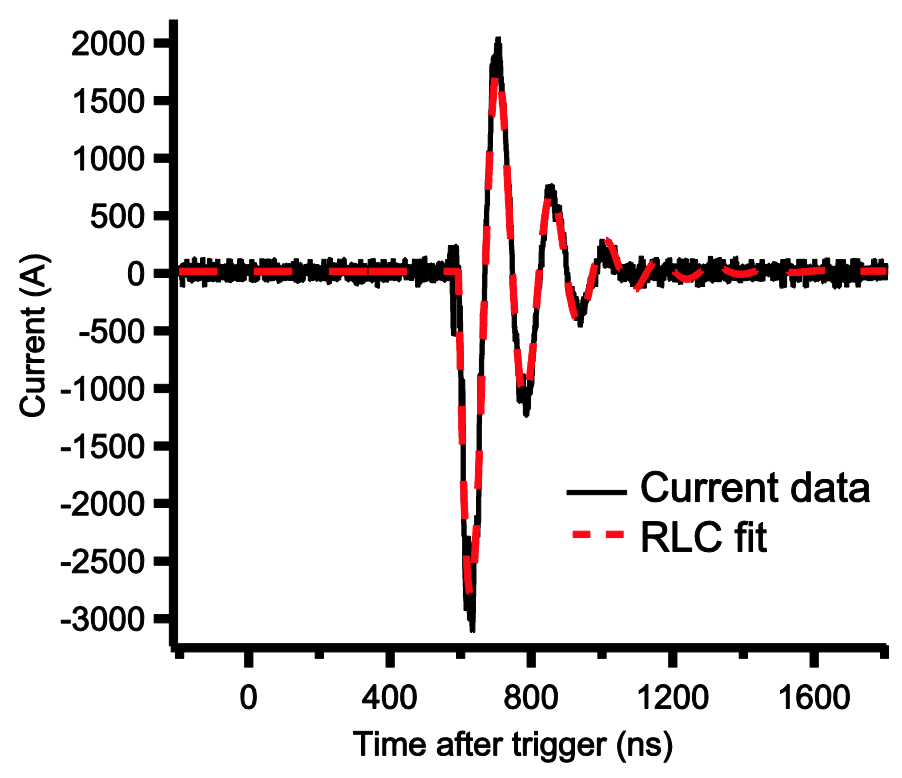

Fig. 2.6: Arc source current measurement (solid black line) with corresponding RLC fit (dashed red line) for an unconfined PETN surface flashover. 

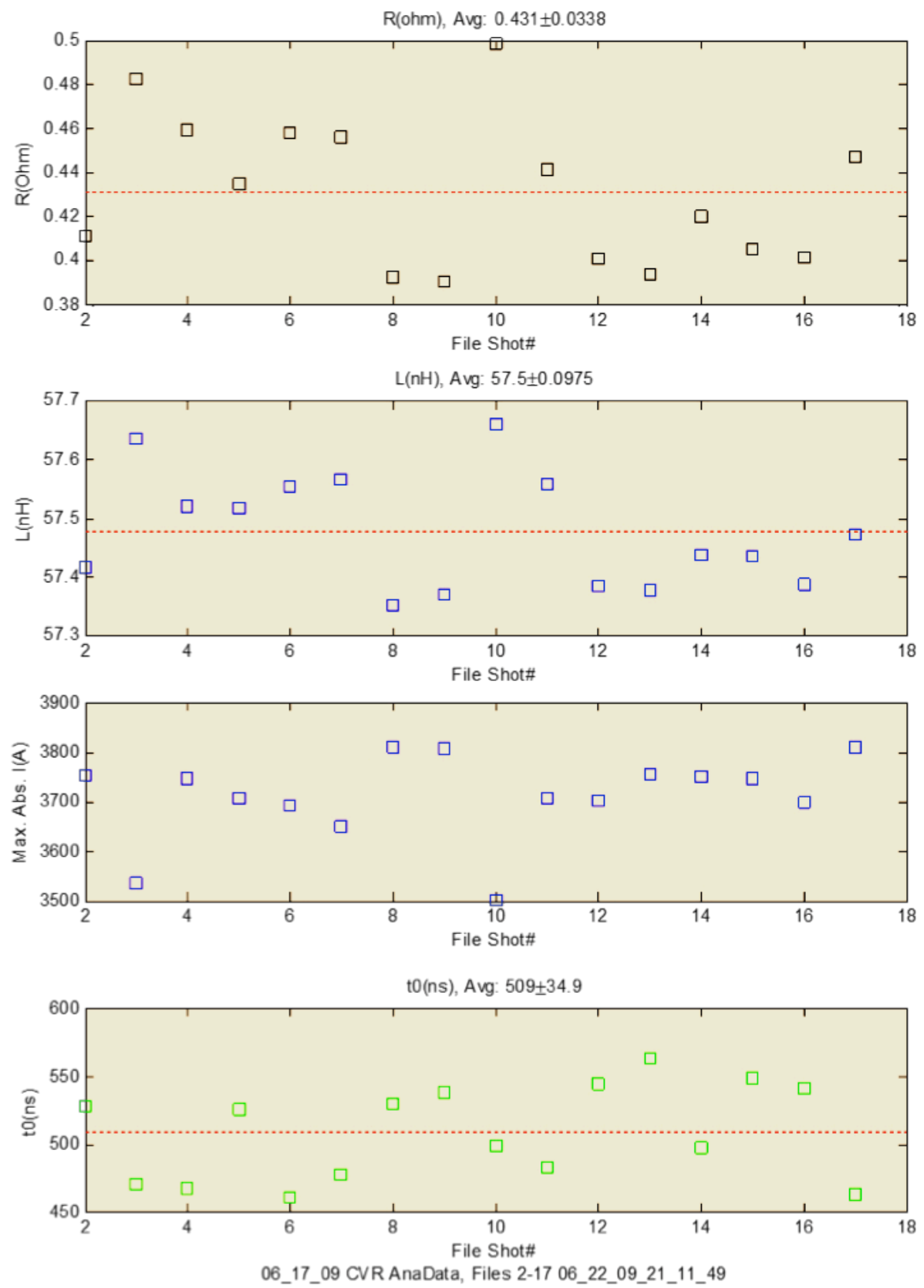

Figure 2.7: RLC fits of a sample series of pulser test shots with a shorted load. The resistance, inductance, maximum current, and firing times are plotted.

\subsection{Revised Holder Design (For TRIR)}

After characterizing the plasmas produced by our fast pulser using the 8-electrode cartridge holder and performing proof-of-principle TRIR experiments, we started work on a more sophisticated load setup that increased the shot rate significantly. The concept behind this "Revised Holder" was to employ a register-able and very long electrode strip, made up of 10's to 100 of single-shot point-to-point electrodes with the same tip geometry as shown in Figure 2.5, in a holder that resembled a film camera. After each shot, the electrode strip is advanced easily 
by rotatable knobs on the holder and registered using the predefined holes in between each pair of electrodes in the strip. Permanent electrodes in the holder are press-fitted to the electrodes on the strip for electrical contact. The substrate window is also press-fitted to the strip on the opposite side and slid down a rail to allow a fresh coating after each shot; the hole in between each pair of electrodes allows the flashover to occur over the substrate window. $\mathrm{A} \mathrm{CaF}_{2}$ reaction chamber, or liner, $2 \mathrm{~mm} \times 2 \mathrm{~mm} \times 1 \mathrm{~mm}$ in dimension was incorporated for confined shots. Figures 2.8 and 2.9 illustrate the concept schematically along with the implementation. Alternatively, the holder could also be operated with just the permanent electrodes without the electrode strip. The complete drawings are available in Appendix F.

During testing with the electrode strip we were able to achieve shot rates of $\sim 30$ shots an hour, with the electrodes automatically achieving alignment to the TRIR system better than $0.15 \mathrm{~mm}$ due to the use of the precise registration holes in between the electrodes tips on the strip. The discharges were point-to-point single filament and reproducible; the main difference in these arcs versus the ones in the clamp load is the liner which provides some confinement. The $\mathrm{CaF}_{2}$ liner showed little to no damage after repeated shots. In this setup, the arc plasma temperatures and densities were measured simultaneously with the TRIR measurements. The arc plasma temperatures and densities were similar as shown and discussed in the following Chapters. The electrical parameters from the RLC fits were similar also. A system inductance of $71 \pm 0.4 \mathrm{nH}$, resistance of $0.728 \pm 0.07 \Omega$, and an averaged peak current of $3.26 \mathrm{kA}$ was found over 22 replicates. The system inductance is approximately $2 \mathrm{nH}$ higher than in the clamp load due likely to the longer transmission line used.

This rapid single-shot load concept is unique for arc discharges and represents a significant engineering advancement in this area.

We additionally tested the holder with just the round permanent electrodes and also a set of "ninja star" electrodes made of tungsten copper with rotatable sharp tips; the purpose of these tests were to see if we could use them for repeatable arcs without the single-shot electrode strip. Figure 2.11 shows typical multiple filament arcs with the round electrodes that were not viable for our experiments (contrast with the point-to-point arcs shown in Figure 2.12). Although the "ninja star" electrodes were better in terms of shot reproducibility and producing single filament arcs, we ultimately could not get reliable $\mathrm{Cu}$ line data needed for temperature and density measurements due likely to both the doubling of the arc length (from $1 \mathrm{~mm}$ to $2 \mathrm{~mm}$ ) and the reduction in electrode ablation from the harder tungsten copper alloy. Because of time constraints we were not able to investigate these issues fully. 

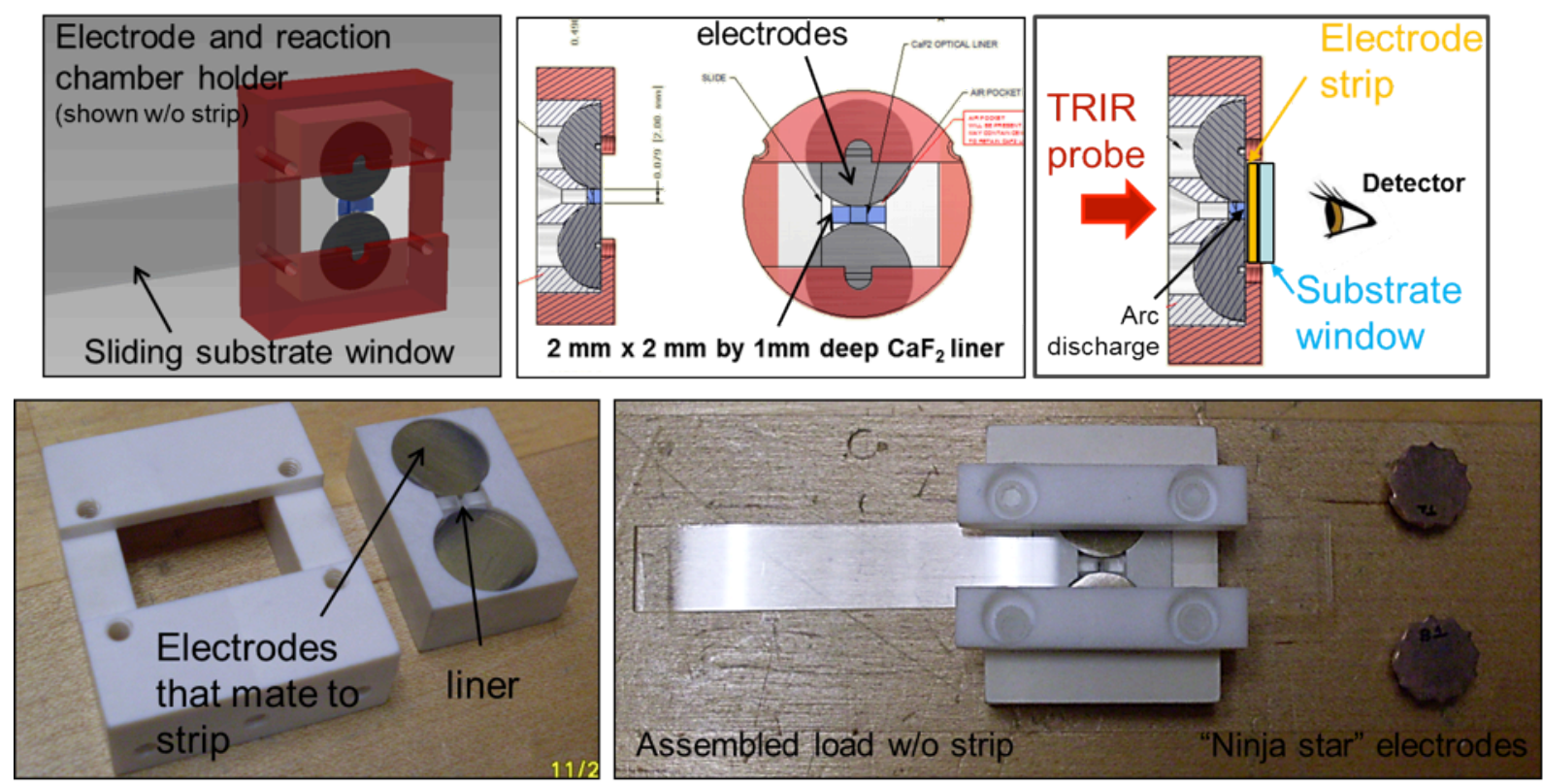

Figure 2.8: Schematic and implementation of the electrode and reaction chamber for the Revised Holder design.
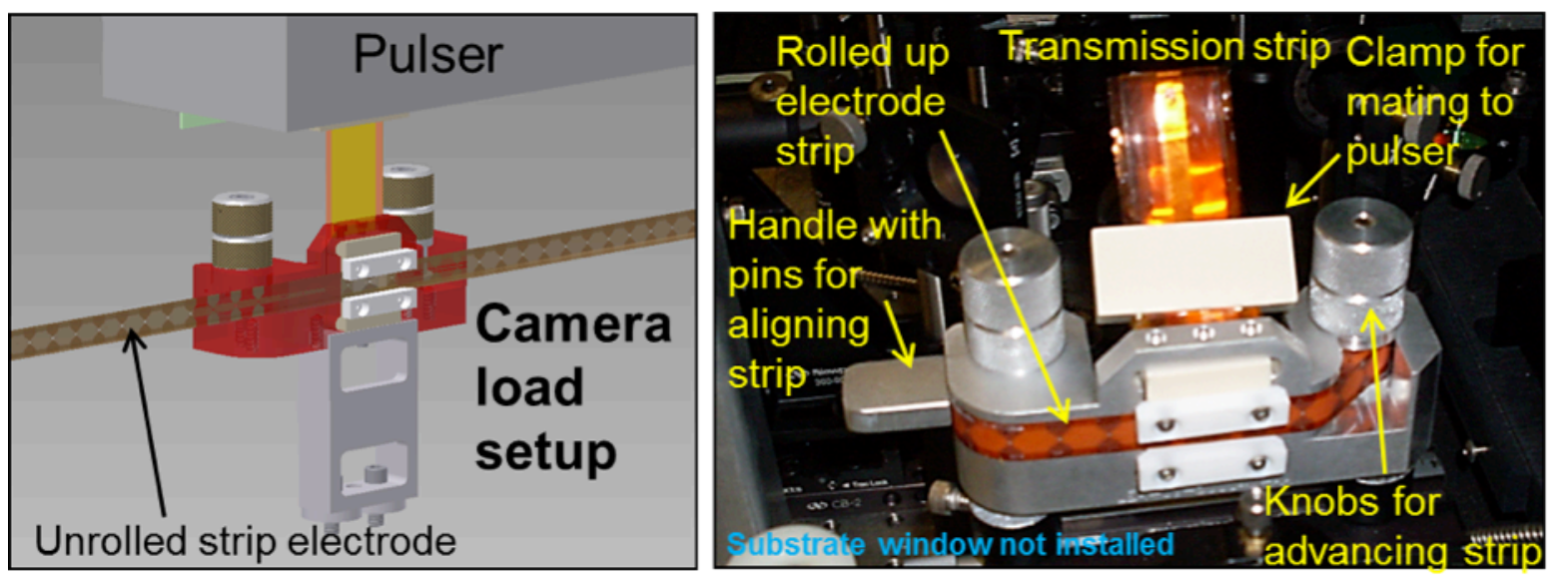

Figure 2-9: "Film Camera" setup for interfacing a long single-shot electrode strip with the reaction chamber in Figure 2-8 and allowing for rapid switching of single-shot electrodes during experiments. 


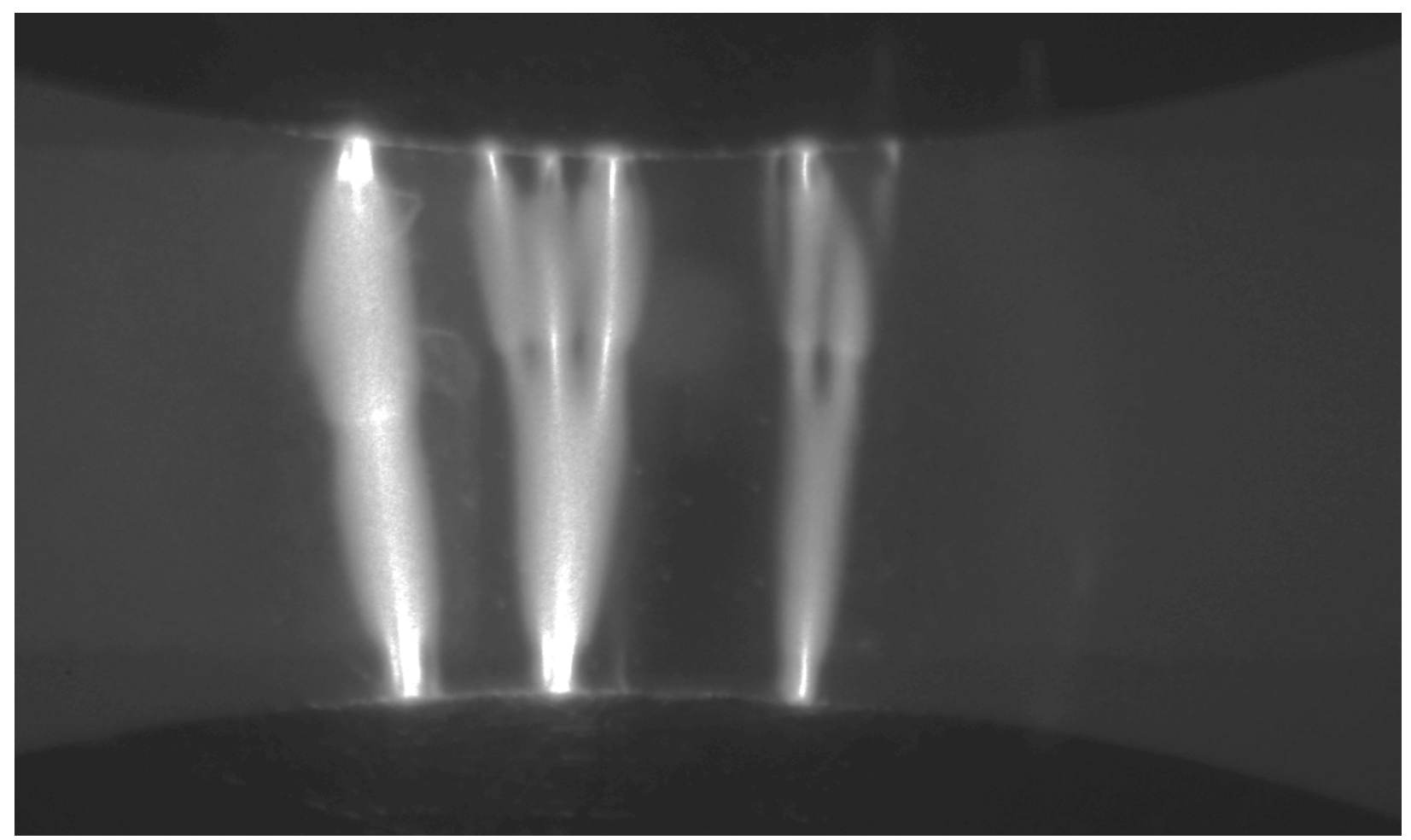

Figure 2.11: Typical multi-filament arc discharge using round electrodes without the single-shot point-topoint electrode strip. The faint outlines of the $\mathrm{CaF}_{2}$ liner can also be seen.
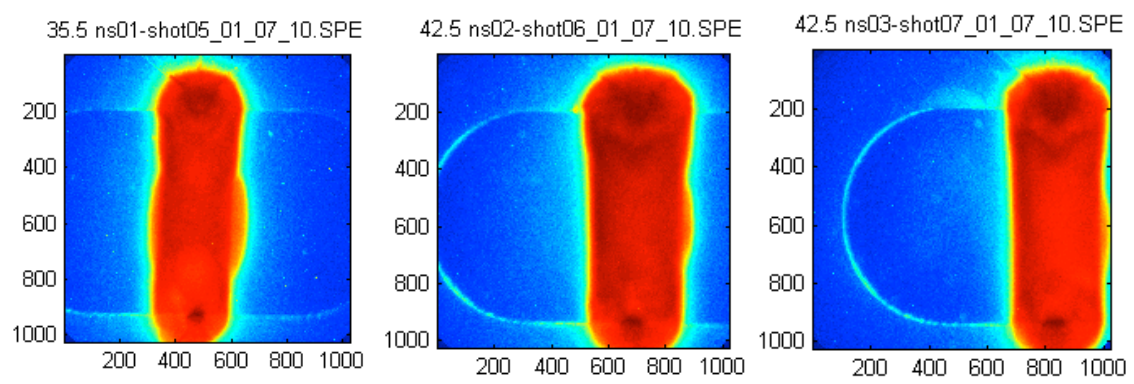

Figure 2.12. Highly repeatable, well-defined arcs produced by the point-to-point single-shot electrodes made from coupons of copper-coated kapton. 


\section{Discharge Characterization with Energetic and Inert Thin Films}

This section is meant to provide a "quick reference" of the characterization work performed on the discharges over thin films of PETN and PMMA. The characterization work is a fundamental ingredient in understanding the stimulus received by the materials of interest and was intended to provide the information necessary to unfold the kinetic data data obtained via TRIR. Even without the eventual coupling to TRIR, however, the characterization work by itself provided some key insights into the arc/HE interaction.

For full details, we refer the reader to the following publications:

- "Towards the Understanding of PETN Initiation by a Fast, High-Power Arc Source," by Chris Grant, Vincent Tang, Elizabeth Glascoe, and James McCarrick was published in the peerreviewed venue of the $14^{\text {th }}$ International Symposium on Detonation, April 2010, pp. 543-551. This is also available through IMS as LLNL-CONF-425208.

- "Spatiotemporal Temperature and Density Characterization of High Power Atmospheric Flashover Discharges Over Inert PMMA and Energetic PETN Dielectric Surfaces," by Vincent Tang, Chris Grant, James McCarrick, Elizabeth Glascoe, and Han Wang, published in the Journal of Applied Physics (J. Appl. Phys. 111, 053303 2012). It is also available through IMS as LLNL-JRNL-505792.

\subsection{Summary of Diagnostics}

Film preparation: The thin films prepared on $\mathrm{CaF}_{2}$ windows were studied using DekTak profilometry to measure thickness and uniformity, and also FTIR to determine the pre-shot absorbance spectra of the films.

During energy input from the pulser: A current-viewing resistor (CVR) was used to measure the output waveform from the pulser. By comparing the shape of this curve to those produced in shorted configurations, we can estimate the power and energy delivered to the discharge.

During and after energy input: A gated spectrometer was used to perform time-resolved emission spectroscopy, in the region of $\mathrm{Cu}$ I lines. Copper enters the discharge via ablation of the electrodes; $\mathrm{Cu}$-doped films were also tried. The relative intensity of the $\mathrm{Cu}$ I series can be used to determine temperature, and the line shape can determine electron density. A gated intensified camera was also used to take visible-light images of the discharge and subsequent plasma motion. The sharp boundaries of the expanding plasma can be used to determine the expansion speed.

Post shot: DekTak profilometry and FTIR were again employed to determine the end-state of the thin films after being subjected to a discharge.

Finally, a one-D hydrodynamic model was used to simulate the behavior of the discharge, taking advantage of the fact that the reactive contribution to the energy is small and therefore not required in the simulation. 


\subsection{Summary of Major Results}

Comparison of pre- and post-shot FTIR spectra shows about a $\sim 300 \mathrm{~nm}$ depletion in all PETN vibrational modes in the portion of the film subjected to the discharge. Visible inspection shows the extent of the region affected; there is no self-sustained propagation. The $\sim 300 \mathrm{~nm}$ value appears to be independent of film thickness, as we measure $\sim 30 \%$ absorbance change in 1 micron films and $\sim 60 \%$ change in $500 \mathrm{~nm}$ films. This implies the current channel does not form in a breakdown of the solid material, but rather is carried in the air above the film.
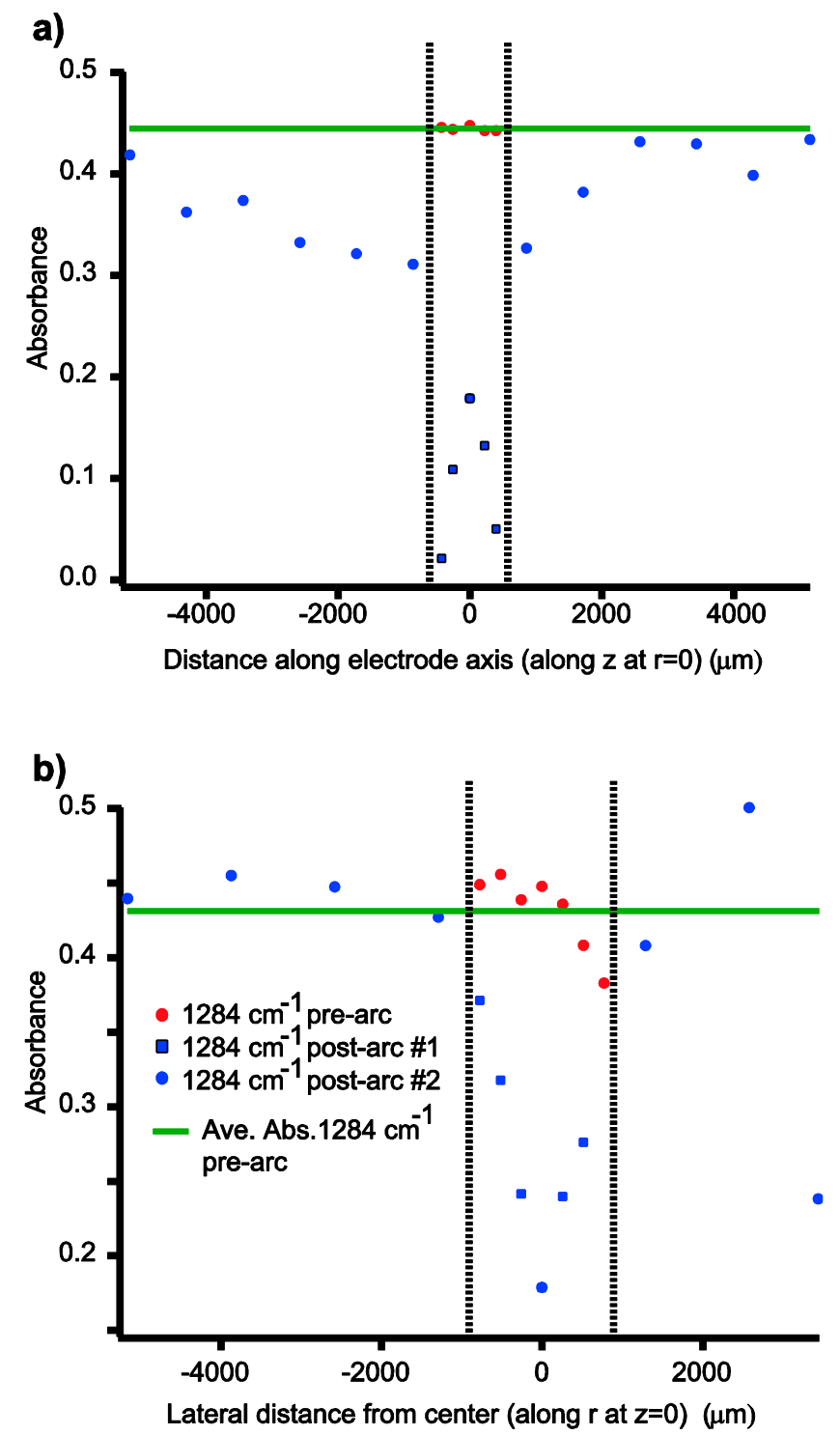

Figure 3.1. Post-shot FTIR analysis of PETN films, both along the $r$ and the z-axis, using the $\mathrm{NO}_{2}$ symmetric stretch $+\mathrm{CH}$ bend $+\mathrm{C} 5$ skeletal combination mode. Approximately $60 \%$ of the material is depleted in the $\sim 1$ $\mathbf{m m} \mathbf{x} \sim 1 \mathrm{~mm}$ plasma region corresponding to $\sim 300 \mathrm{~nm}$ of PETN material. 


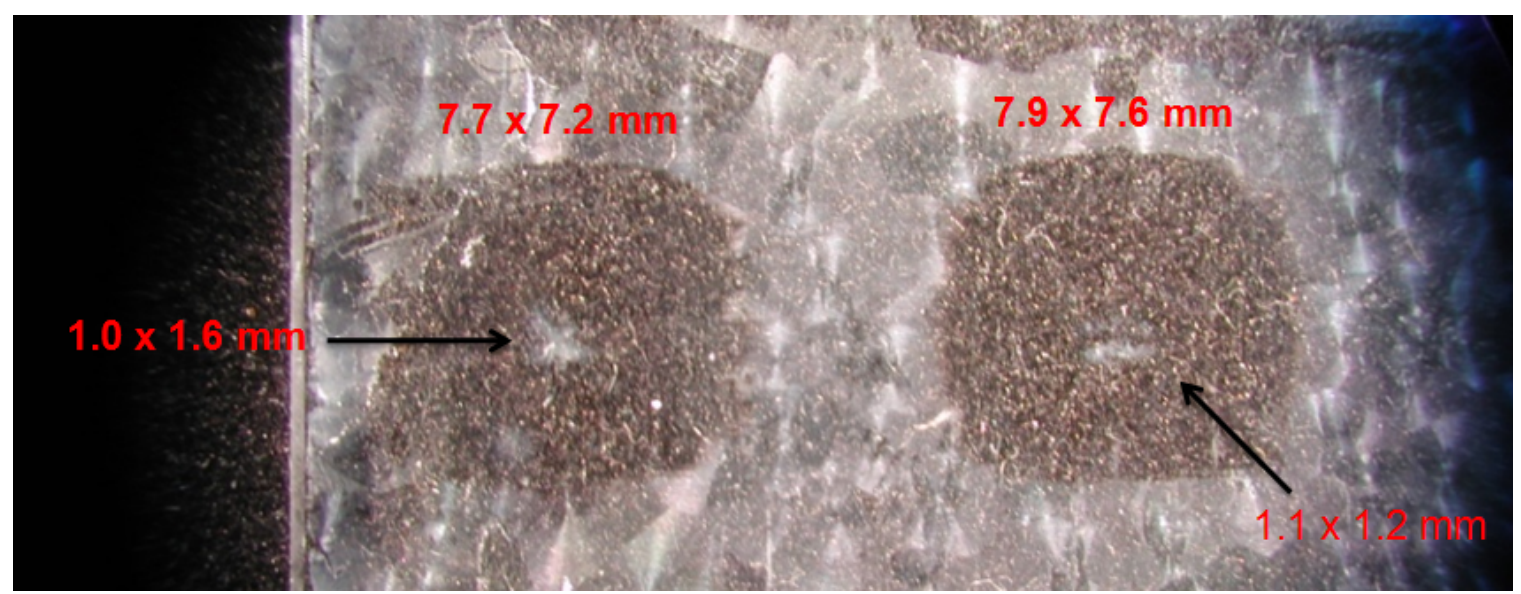

Figure 3.2. Post-shot images of the film coatings.

Determination of the time-resolved temperature finds a quick rise to a temperature of several $\mathrm{eV}$, which decays over several microseconds. An $0.5-1 \mathrm{eV}$ "tail" persists out beyond 10 microseconds. The peak electron densities approach $10^{18} / \mathrm{cc}$, with a temporal decay similar to the temperature. Densities of order $10^{17}$ persist beyond 10 microseconds.
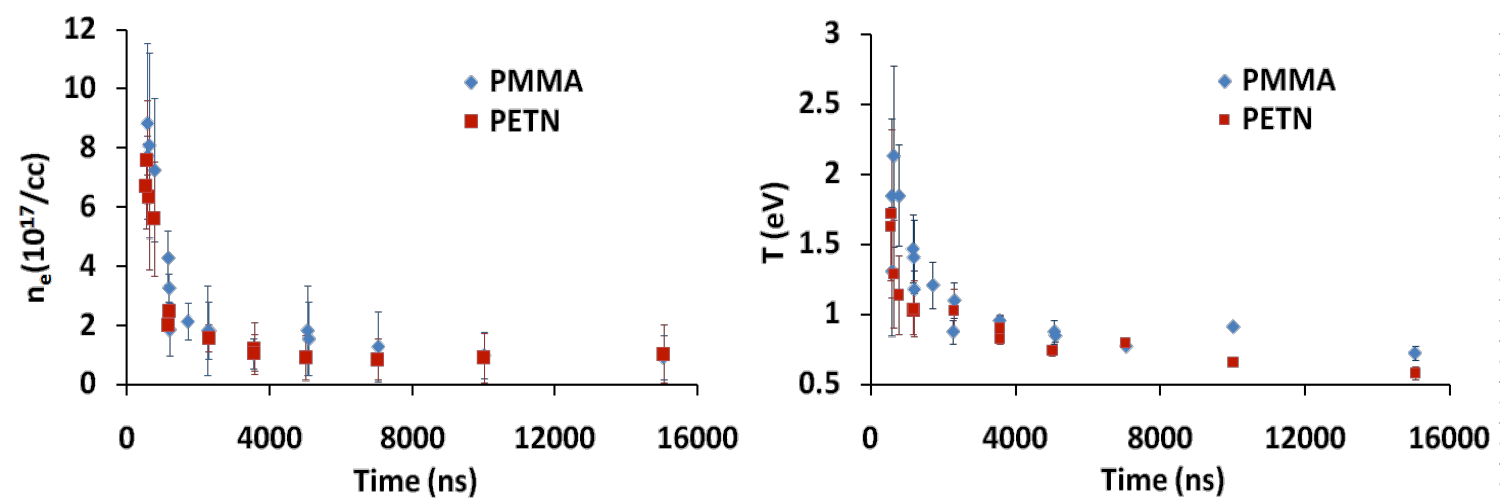

Figure 3.3. Time dependence of the electron density (left) and ion temperature (right), at the center of the discharge.

Gated images of the visible light emission show a sharp expansion front that persists until the plasma radius exceeds the electrode spacing. The initial streamer is straight (no branches or kinks) and of the same order as the electrode tips in size. 

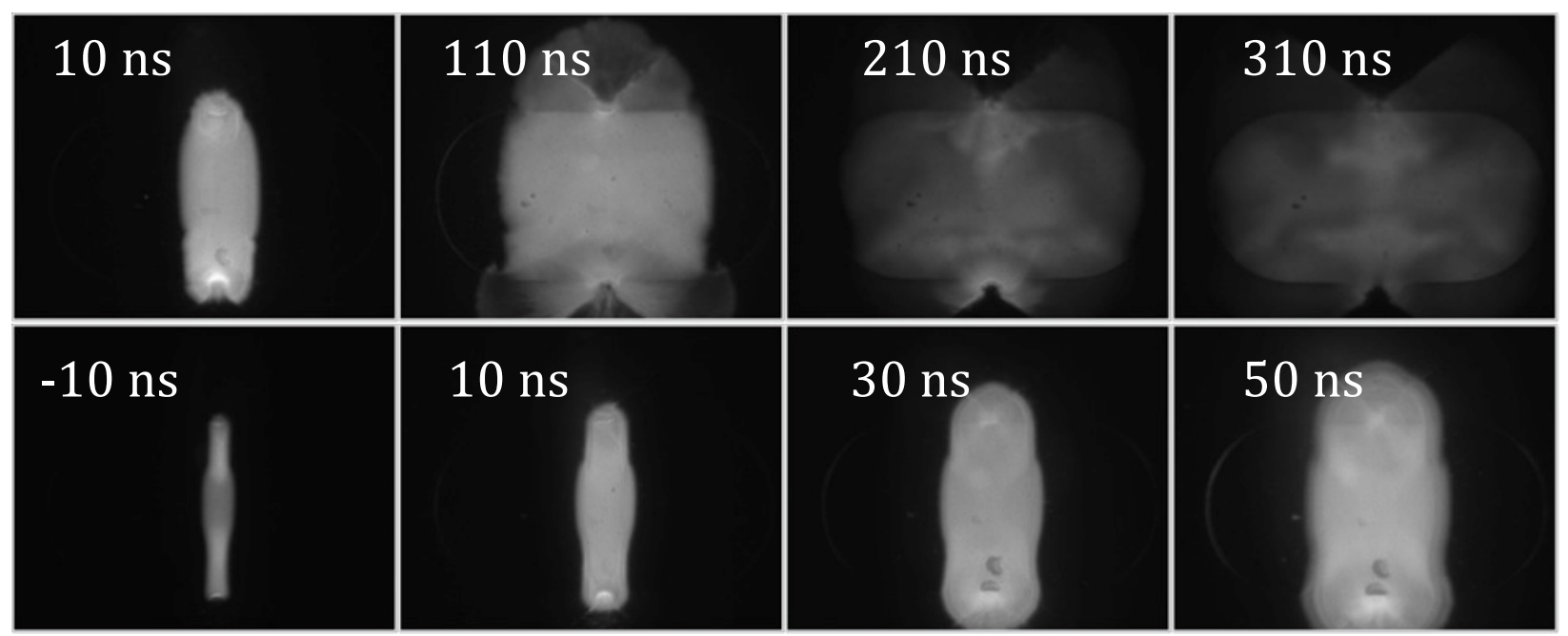

Figure 3.4. Visible light images of the discharge, taken with a 3 ns gate.

Performing measurements at fixed time but at different radii shows that, behind the expansion front, the spatial profile of temperature and density are relative flat.
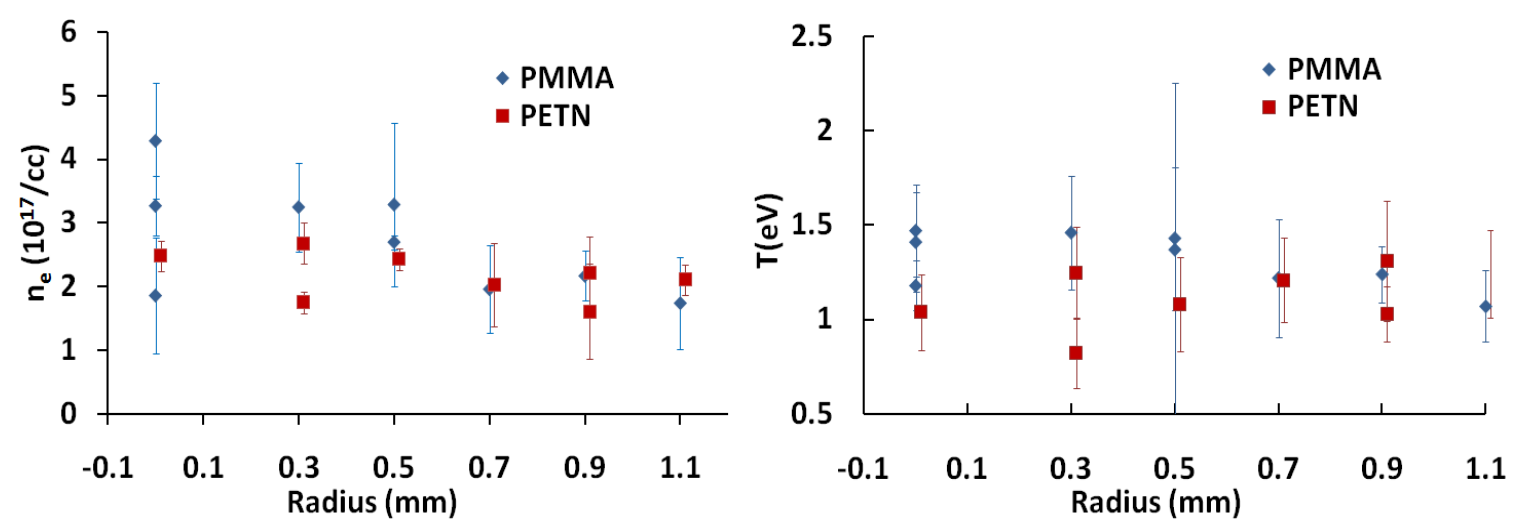

Figure 3.5. Spatial profiles, using data taken at the same times during discharge evolution.

Simulations of a 1-d cylindrical expansion are able to closely reproduce the motion defined by the sharp edge of the visible emission. The EOS is ideal but joule heating, radiation emission and loss are included. 


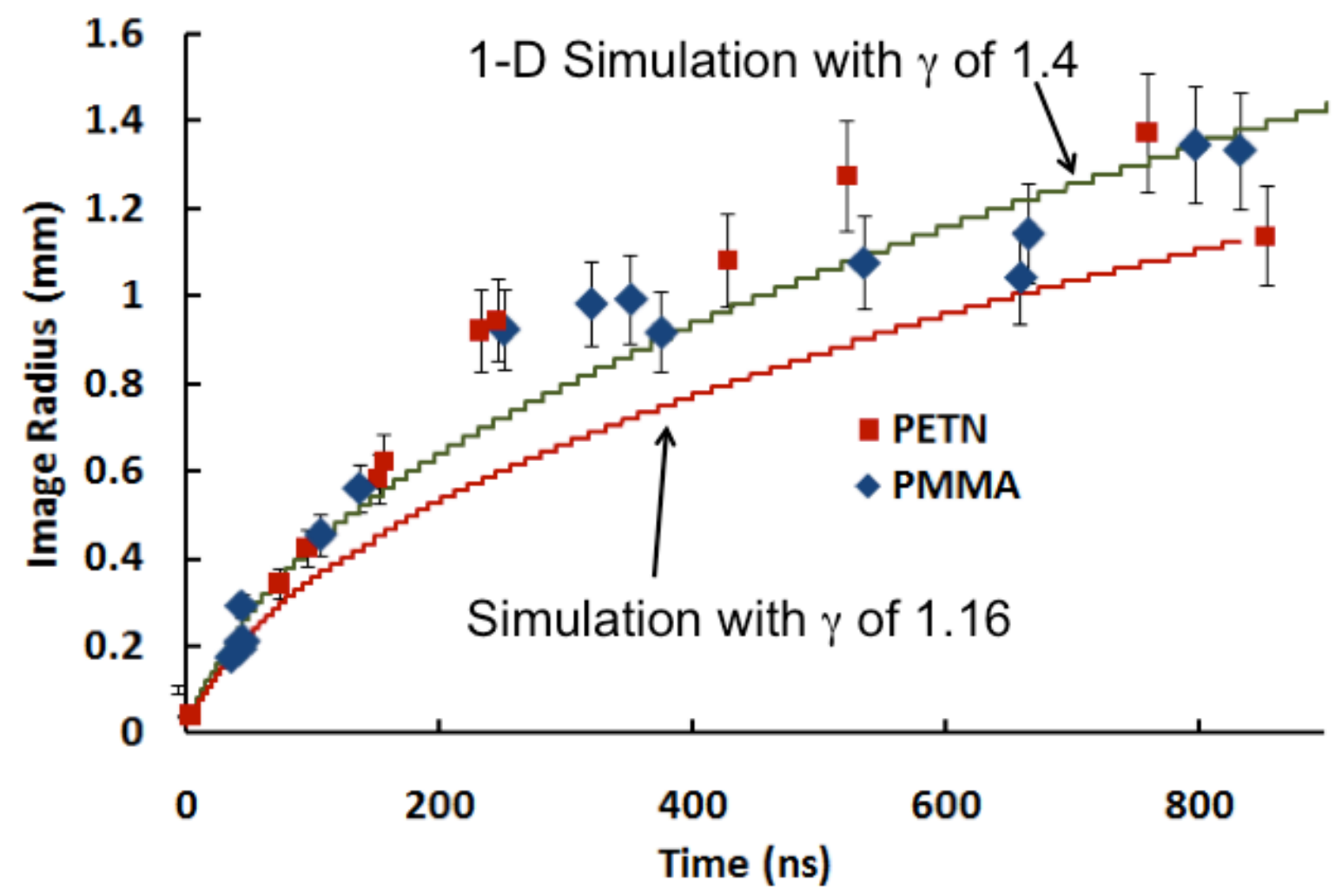

Figure 3.6. Comparison of the edge radius over time with a 1-d cylindrical hydro model. 


\section{Time-Resolved Infrared Spectroscopy of Thin Films}

\subsection{Methodology}

The chosen technique for following high-temperature chemistry of high explosive molecules induced by an arc discharge was time-resolved infrared spectroscopy (TRIR). This technique can monitor specific chemical bonds potentially allowing for the determination of the chemical reaction pathways and their associated time scales for formation/depletion. TRIR has been used to monitor TATB deflagration chemistry and was reported previously [Glascoe, JPCA, 2009]. Here we attempt to apply TRIR to help elucidate surface flashed-PETN chemistry. A schematic is shown in Fig. 4.1 while a photograph of the optical table is shown in Fig. 4.2.

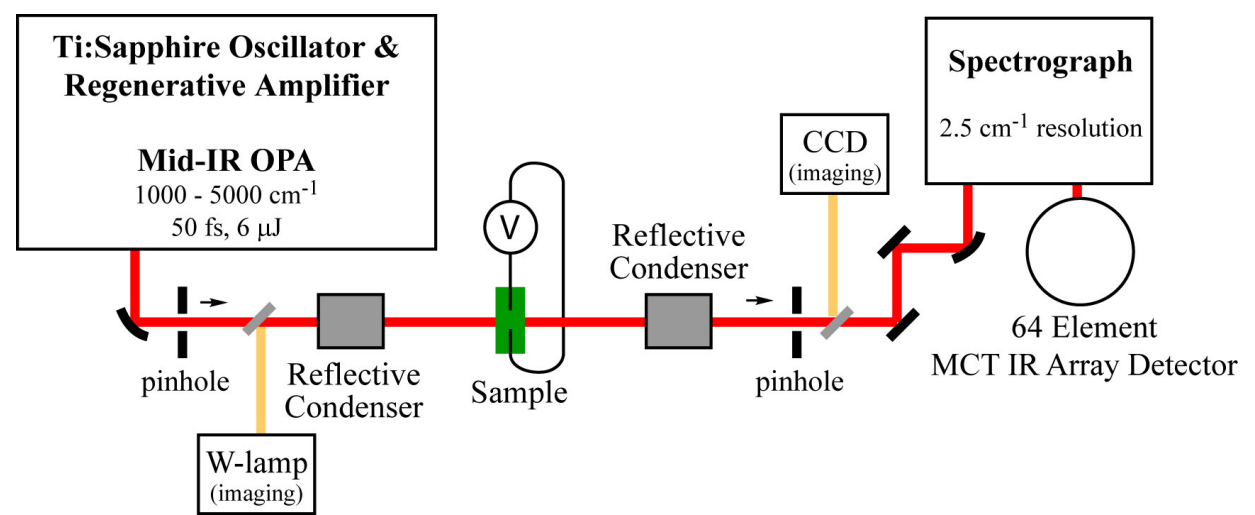

Figure 4.1. Schematic of the TRIR system.

Briefly, 50 fs pulses of mid-IR light $\left(1000-5000 \mathrm{~cm}^{-1}\right)$ are generated by an optical parametric amplifier (Coherent, Opera) pumped by the output of a Ti-Sapphire regenerative amplifier (Coherent, Legend) that is seeded by a Ti-Sapphire oscillator (Coherent, Mira). IR pulses are focused through an alignment iris by a gold-coated off-axis optical parametric mirror and directed into a Cassegrain objective. This objective refocuses the IR beam to a $\sim 20 \mu \mathrm{m}$ beam diameter. Another identical Cassegrain objective collects the transmitted light and focuses it through a second spatial alignment iris. Finally, the beam is focused via a gold-coated off-axis optical parametric mirror into a spectrograph (Jobin Yvon, iHR320) and detected using a 64element liquid nitrogen cooled $\mathrm{HgCdTe}$ (MCT) infrared-array detector (InfraRed Associates, Inc.) coupled to a high-speed signal acquisition system (Infrared Systems Development Corp.). The spatial resolution of the spectrometer, using a 58 grooves $/ \mathrm{mm}$ grating, is $3 \mathrm{~cm}^{-1} / \mathrm{pixel}$ at $3000 \mathrm{~cm}^{-1}$ and $0.5 \mathrm{~cm}-1 /$ pixel at $1400 \mathrm{~cm}^{-1}$. For these inherently single-shot experiments, a digital delay system synchronizes the IR pulse and the arc pulser with the detector electronics. 


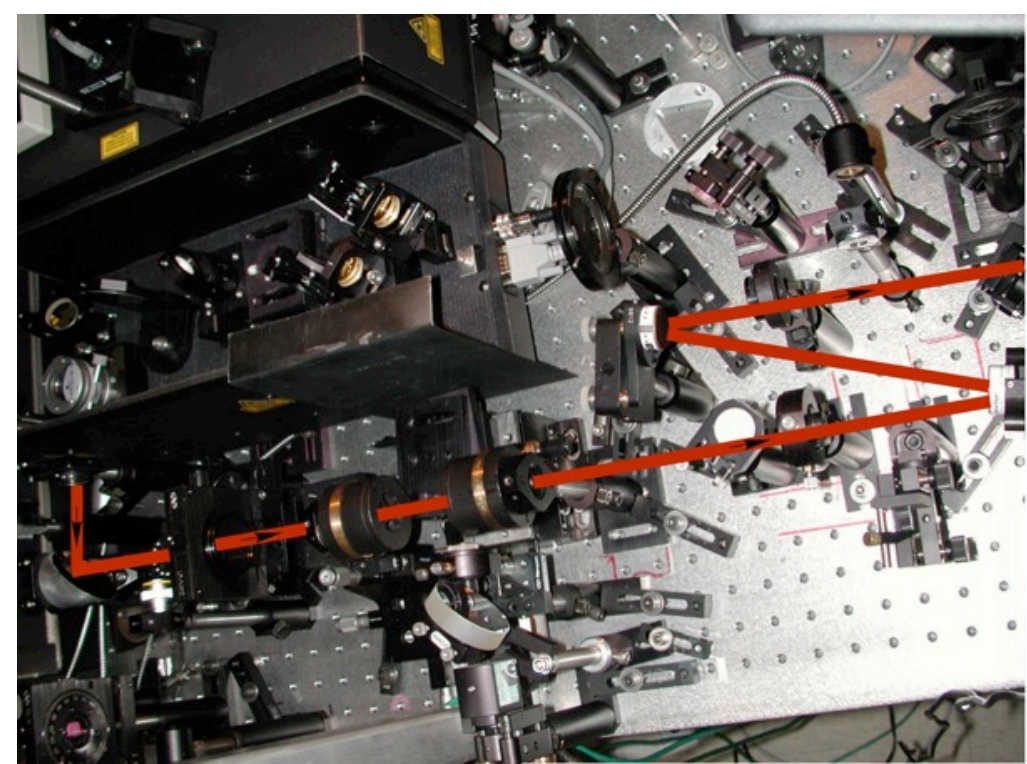

Figure 4.2. Photograph of the optical system showing the direction of the laser light from the mid-IR OPA system (top left) towards the MCT detector (not pictured to the right).

\subsection{Results, First Campaign}

The first TRIR campaign used the initial eight-electrode coupon variant of the clamp system discussed previously. This clamp was attached to a rail which itself was affixed to a X-Y translation stage that allowed for positioning of the HE sample relative to the fixed laser beam. Visualization of the sample/electrode area was accomplished using a CCD based imaging system (see Fig. 4.1). The sample was positioned so that the laser beam was approximately $500 \mu \mathrm{m}$ from either electrode and along the electrode centerline. The strip line would then be clamped to the pulser via set screws just prior to functioning of the pulser. After the sample was arc insulted, the clamp was moved along the rail to the next fresh electrode and the process was repeated till all eight electrodes had been used at which point a new film/electrode coupon assembly would be built and mounted.

The duty cycle to function eight shots varied, but, on average, eight fresh shots could take approximately 4 hours to collect. This was mainly due to the constraints inherent in the design of the coupon/clamp/pulser interface that was driven initially by the need to keep inductance low in order to deliver the necessary amount of energy to the arc and HE surface on the shortest time scale as possible. This ultimately meant the pulser and clamp were very closely coupled spatially. That constraint along with the positioning of various optics made working conditions cramped.

All spectra were collected in the following manner. A background spectrum was collected usually averaging 100-1000 laser shots in the absence of any laser illumination that was then stored in the LabView data collection program. A reference transmission spectrum on an uncoated $\mathrm{CaF}_{2}$ window was collected also averaged over 100-1000 laser shots and saved for post data collection processing. A pre-sparked film spectrum was collected using 100 averaged spectra while the transient was a single spectrum. For comparison between with pre- and transiently sparked films, a spectrum was also collected post-sparked insult and again averaged 
over 100 shots. All spectra were transformed into absorbance before analysis. Pre- and transiently sparked spectra were fit to Gaussian profiles and background subtracted to determine peak areas.

Timing was selected by using a digital delay generator and varied when the arc pulser fired relative to when the laser pulse arrived. Output from a single pixel on the MCT IR detector was routed into an oscilloscope along with the CVR output from the pulser. The difference between the start of current flow and the arrival of the laser pulse yields the actual time delay for any given shots. The type of data is illustrated in Fig 4.3. Also illustrated is the amount of signal induced by the high power arc pulser in the IR MCT detector that lasts out to approximately 3 $\mu s$. This result was typical from shot to shot. It is evident that the relative amount of noise induced by the pulser is much larger than what the detector sees from the actual laser pulse. This amount of noise made data collection at times relative to pulser current flow earlier than $\sim 2 \mu \mathrm{s}$ impossible. However, even at later delay times, pulser induced noise in the IR detector was persistent even though this did not manifest itself in the IR MCT pixel readout. Another interesting observation about the noise inducing or broadcasting nature of the high power arc pulser is the fact that on multiple occasions the IR spectrometer's grating moved immediately after functioning the pulser. These incidents happened with enough frequency that after the spectrometer was moved to the appropriate wavelength for data collection, the spectrometer was powered down to prevent any subsequent grating movement during data collection.

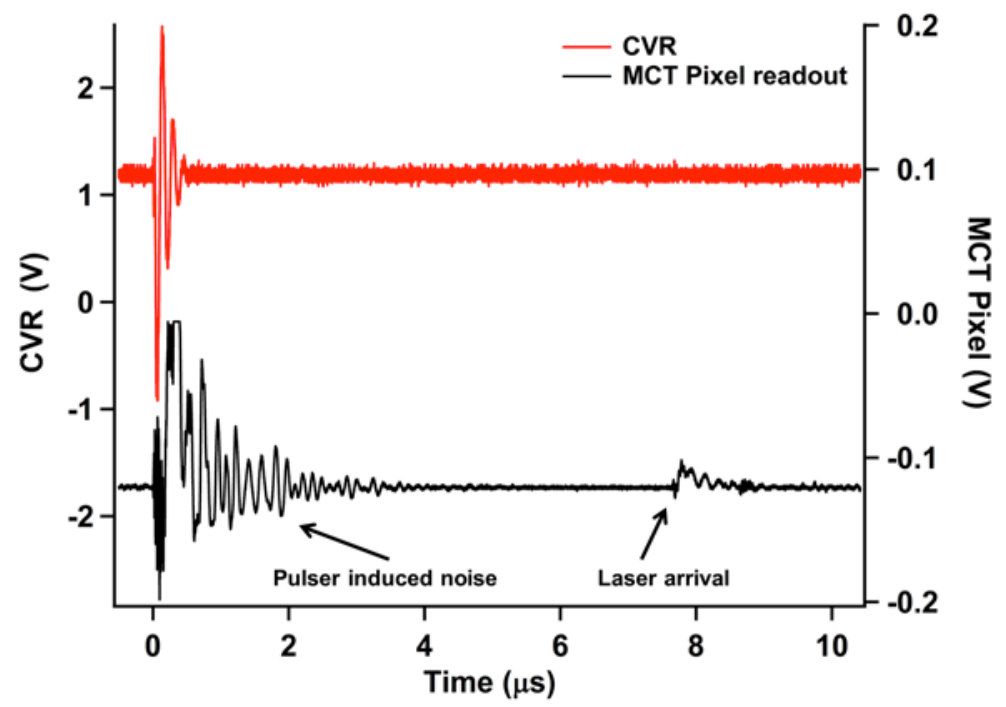

Figure 4.3. Waveform traces from the arc pulser CVR and from one of the IR MCT detector pixels. The timing for the TRIR is determined from the start of current flow from the pulser and the rise in current from the arrival of the IR laser pulse. In this case, the delay is $\sim 8 \mu$. Also of interest is the amount of noise induced in the IR detector by the current flow in the arc pulser. Traces are offset from each other for clarity.

Nevertheless, in spite of these constraints we were still able to collect some usable data from our first campaign by avoiding the first few $\mu \mathrm{s}$, but it took approximately 20 shots to gain only three usable time points. In any case, in Figure 4.4a, we present preliminary time-resolved IR absorption spectra (difference between the pre-sparked film and the transient) of an unconfined PETN film for three different time delays relative to plasma development plus an infinite time delay spectrum using the $\mathrm{NO}_{2}$ asymmetric stretching vibrational modes around $1650 \mathrm{~cm}^{-1}$ 
[Gruzdkov, JPCA, 2004; Folz, $10^{\text {th }}$ Det. Symp., 1996].These particular modes were chosen because they are the most intense IR modes for PETN and also because reactions of nitrate esters typically involve the $\mathrm{NO}_{2}$ moiety - e.g., the dominant reaction under ambient conditions is the homolysis of the $\mathrm{O}-\mathrm{NO}_{2}$ bond while under high pressure or shock loading heterolysis forming the carbocation and the nitrate ion is observed [Davis, JPC, 1996; Dreger, JPCB, 2002; Hiskey, JPC, 1991; Naud, J Org Chem, 1992]. The infinite time delay spectrum was taken at least several seconds after the sample was sparked. At $2 \mu$ s, we observe $\sim 0.2$ O.D. depletion of PETN while the change in absorption at $6 \mu$ s and $8 \mu$ s are nearly identical and almost overlay with the infinite time delay spectrum. If we compare the normalized change in absorption versus time as displayed in Figure 4.4b and make the very simple assumption of first order kinetics, we can estimate a $1 / e$ time constant of $\sim 2 \mu$ s.

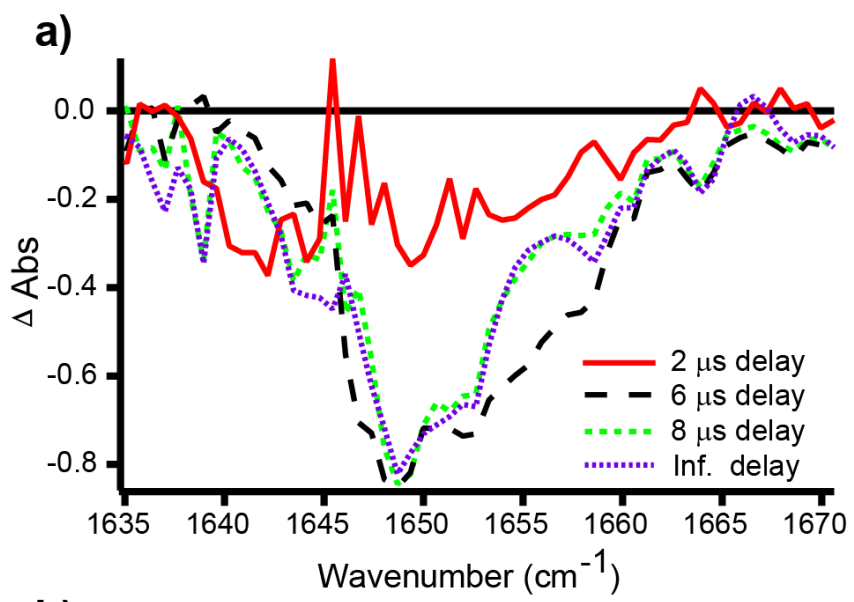

b)

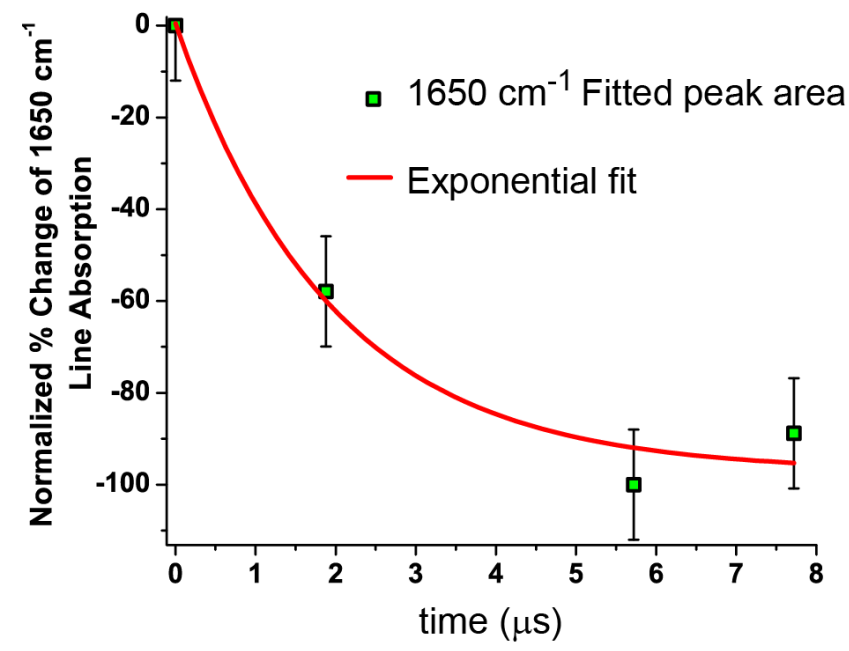

Figure 4.4. a) Time-resolved IR difference spectra between the pre-sparked film and the transient of an unconfined PETN film for three different time delays relative to plasma development plus an infinite time delay spectrum using the asymmetric NO2 stretching mode region. b) Normalized \% change showing the dynamics of PETN depletion. Assuming simplistic first order kinetics an exponential fit yields a decay constant of $\sim 2 \mu$ s which sets a time scale for the reaction/depletion of the energetic material. 


\subsection{Preliminary results, second campaign: noise issues}

Prior to the start of the second campaign, the TRIR system was redesign to improve laser as well as overall shot throughput. Images of the redesigned system are shown in Fig. 4.5. This redesign included removal of the Cassegrain optics and replaced with $\mathrm{CaF}_{2}$ lens to focus the IR light through the sample chamber. This had the effect of increasing the laser spot size by a factor of approximately 10 from $\sim 20-50 \mu \mathrm{m}$ to $\sim 400 \mu \mathrm{m}$. The redesign also utilized the new coupon clamp system termed the "camera clamp", which has been described in detail previously in this document and provided a much higher shot-to-shot throughput than the previous eight electrode coupon clamp design. Further, an optical path was built so as to pick off some of the arc light using a $\mathrm{CaF}_{2}$ window and directed to the Andor spectrometer to collect $\mathrm{Cu}$ atomic emission for temperature determination. The surface of the $\mathrm{CaF}_{2}$ window was sputter coated with $\mathrm{Al}$ making a highly mirrored finish. However, the center of the aluminized window was etched away to allow IR light pass into the TRIR spectrometer. This redesign allowed simultaneous measurement of both atomic emission for temperature determination and time-resolved infrared data to monitor chemical dynamics. 


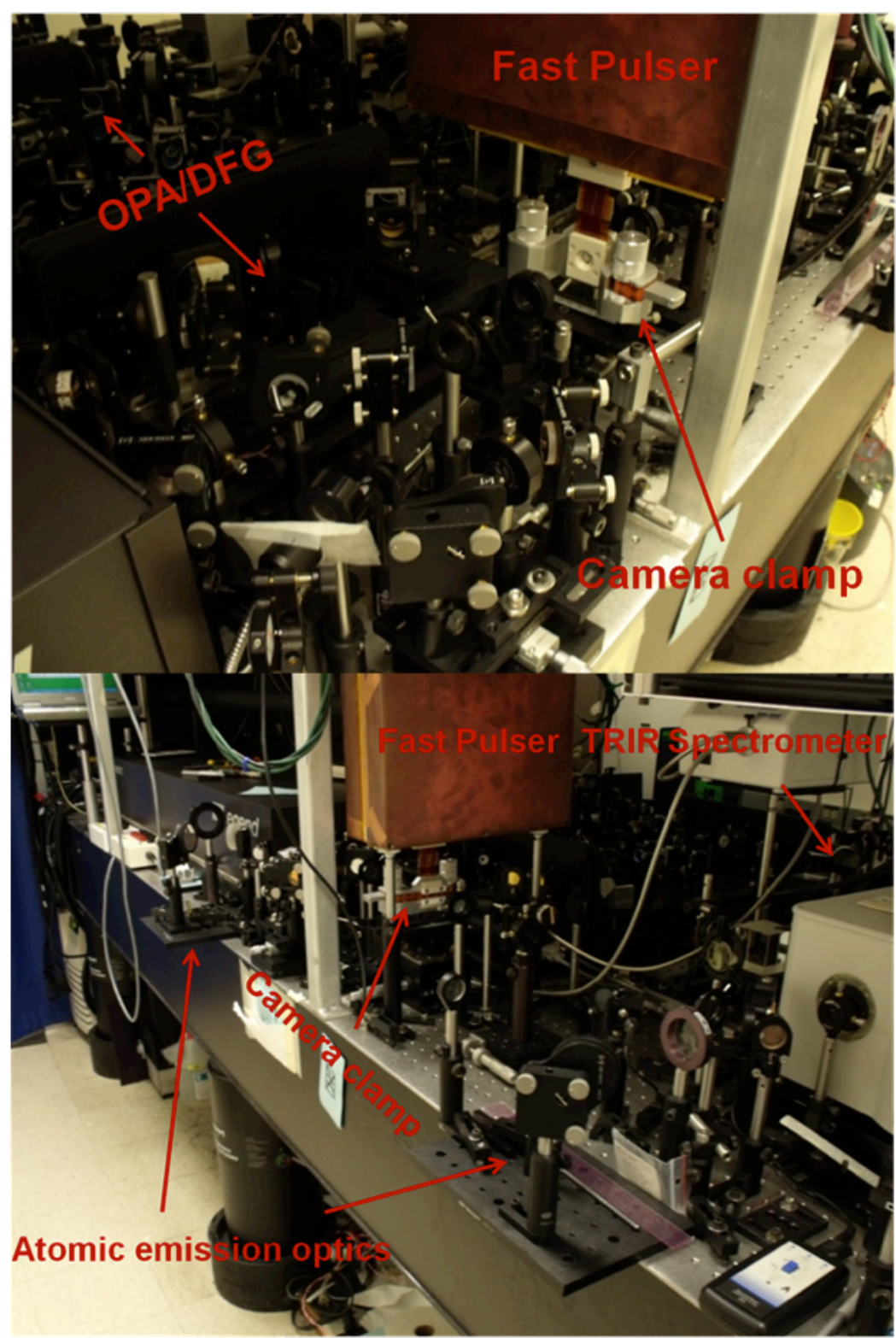

Figure 4.5. The redesigned TRIR system showing the various optical and electrical components. Mid-IR laser pulses are directed from the OPA/DFG system (left to right in both images) through the camera clamp and into the TRIR spectrometer. The atomic emission optics direct part of the arc plasma light down the optical bench to a fast gated detector for collection.

Figure 4.6 shows a close up image of the camera clamp containing a PETN thin film sample. It is clearly evident in the photograph round spots that are the result of HE being ablated or otherwise reacted in response to the arc discharge. This was convenient in that it allowed the operator a visual mark with which to move to an undamaged part of the film for the next experiment. The new clamp design was a success in that it greatly increased the shot throughput by a factor of at least 3 . More than 20 shots could be completed within just a couple hours versus 8-10 shots over $\sim 4$ hours with the previous clamp system. 


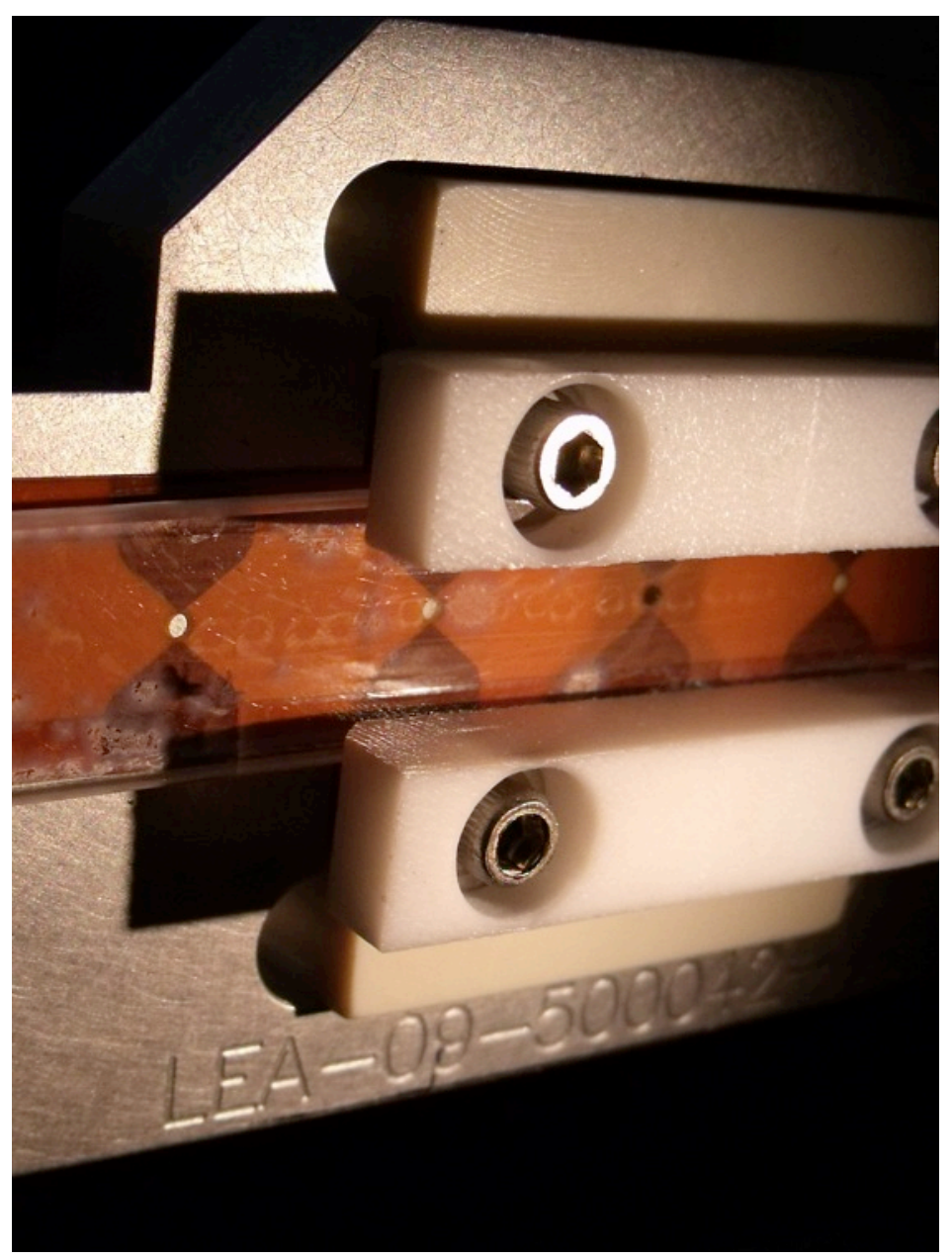

Figure 4.6. Photograph of the camera clamp with a PETN film in place. The round discolored spots are where HE has been reacted/ablated; these spots line up with the circular openings that correspond to the electrodes.

Figure 4.7 shows sample TRIR spectra for two time delays relative to plasma development. It shows both pre- and transient spectra illustrating the time dependent change in area and intensity. Both transient spectra show an overall peak area decrease relative to and are noisier than the prespark spectra. One reason that pre-spark spectra are cleaner is due to averaging while the transients consist of only one spectrum. However, in the $7 \mu$ s delay data there is a clear spike that is likely due to electrical noise introduced by the high power arc pulser. The noise issue will be discussed more below. 

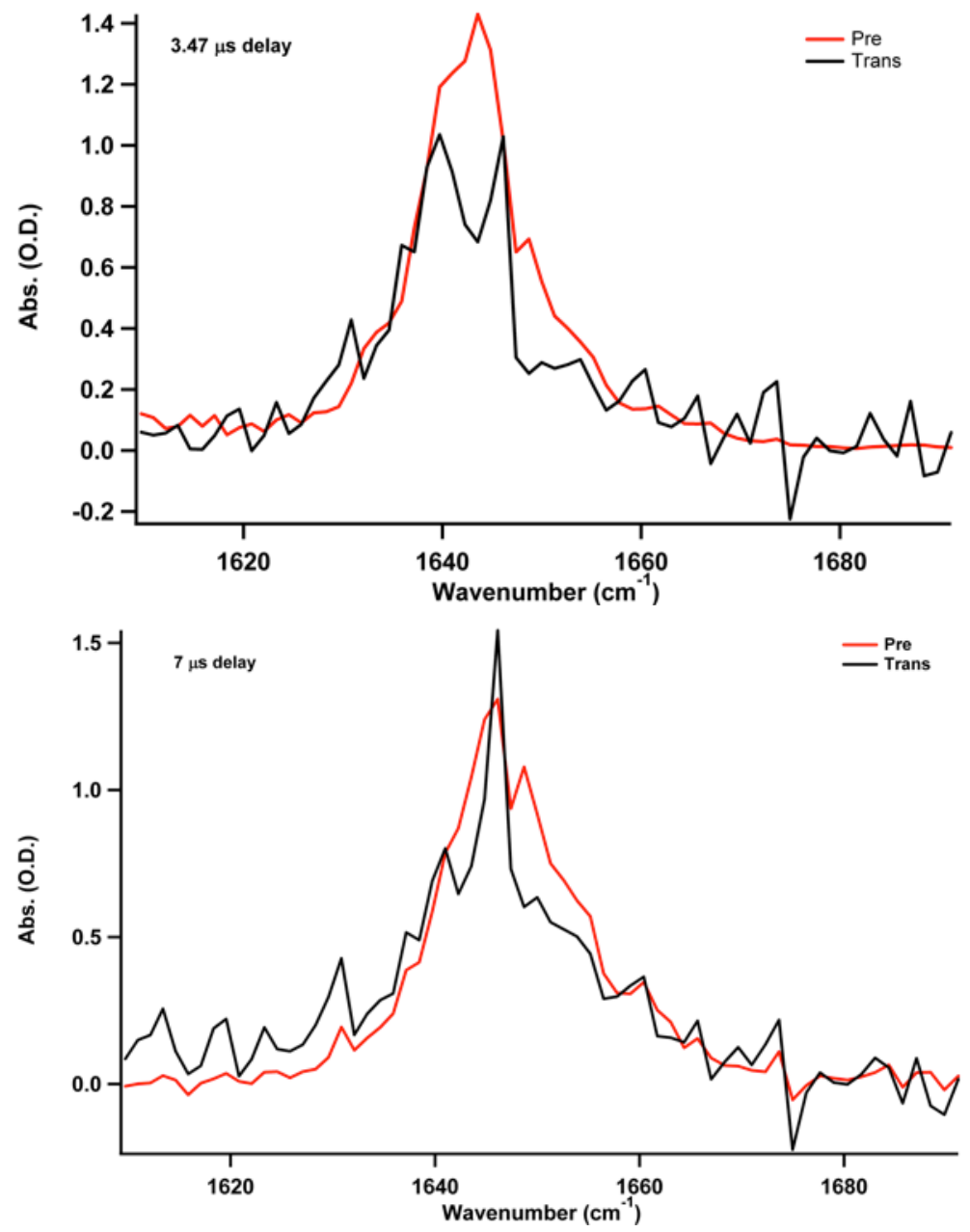

Figure 4.7. Pre- and transiently sparked IR spectra at two different time delays relative to plasma development.

For the second TRIR campaign, 42 data points were taken at different time delays typically with three replicates at each delay. All pre- and transient data were fit to Gaussian lines hapes; the resulting difference in peak areas are averaged and plotted in the top panel of Figure 4.8. There is a fair amount of spread in the data though there is a clear trend as a function of time. If we make the overly simple assumption of first order kinetics like was made previously, an exponential fit yields a time constant of $1.7 \mu \mathrm{s}$, which is in agreement with what was observed for a much smaller number of points from the first TRIR campaign. These data combined with what was obtained in the first campaign sets an approximate time scale for the ablation/reaction of the thin HE film.

In addition, temperature measurements were simultaneously collected during the TRIR shots and the average for each time delay are presented in the bottom panel of Figure 4.8. The temperature ranged from $1.3 \mathrm{eV}$ at $\sim 2 \mu \mathrm{s}$ delay to $0.5 \mathrm{eV}$ at $\sim 8 \mu \mathrm{s}$. At time delays greater than $8 \mu \mathrm{s}$ from plasma development, there was not enough signal intensity from $\mathrm{Cu}$ atomic emission to make a reliable temperature determination. However, we have successfully demonstrated that we are able to monitor chemical decomposition as well as temperature in a time-resolved manner (bearing in mind the limitations of the TRIR system), which in and of itself is an achievement. 
To our knowledge, no simultaneous measurements of this type have been reported in the open literature, and we do plan on publishing our results as soon as possible.
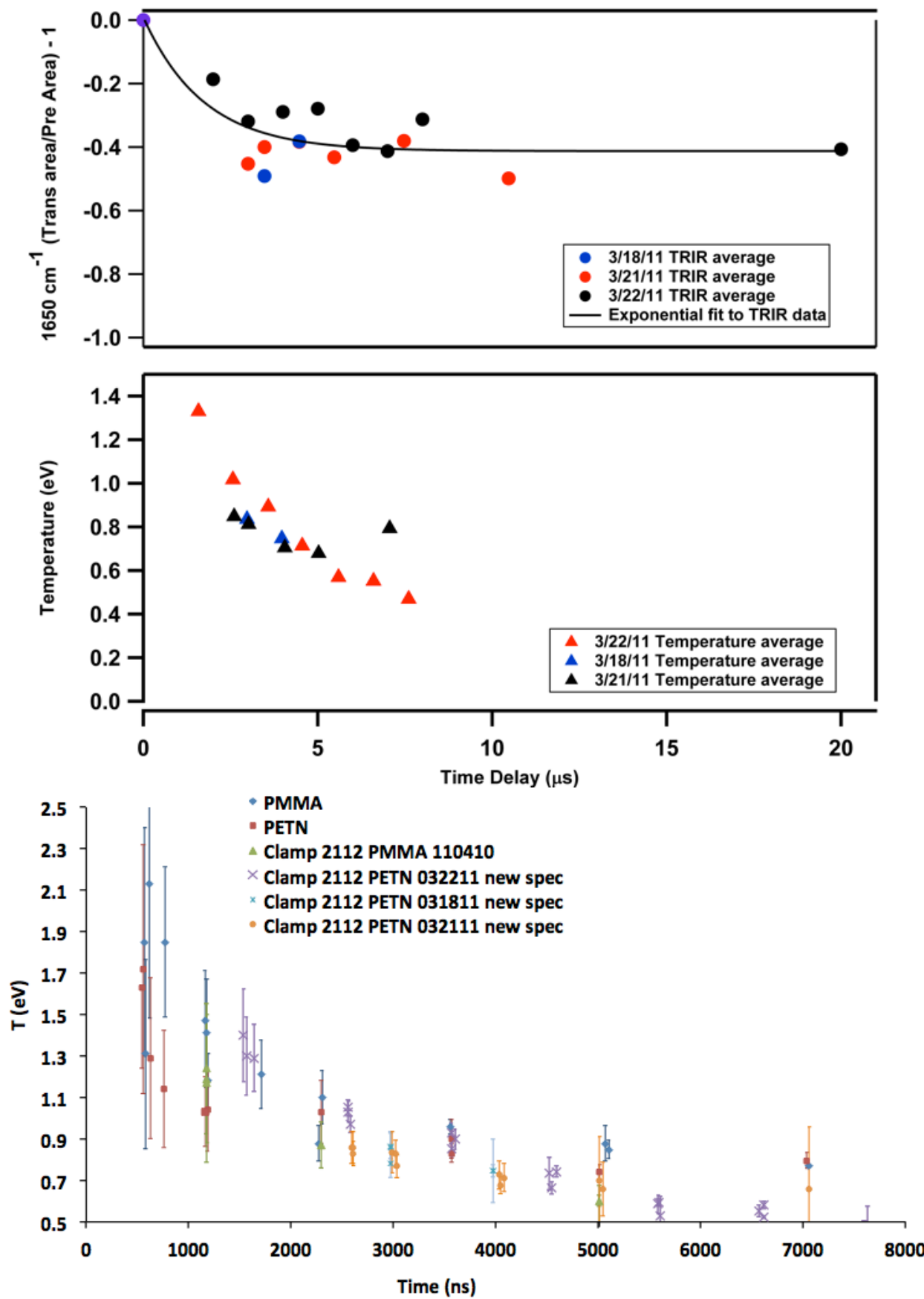

Figure 4.8. Top: Summary of TRIR $2^{\text {nd }}$ campaign data. The changes in peak area between pre- and transiently sparked spectra have been average for each time delay. The number of total shots that make up this figure is 42 . Making the overly simple assumption of first order kinetics an exponential fit to this data yields a time constant of $1.7 \mu \mathrm{s}$ which is consistent with the previous results from the $1^{\text {st }}$ campaign. Middle: Summary of temperature data simultaneously obtained with the TRIR measurements. Temperature data presented are average for each time point. Beyond $8 \mu \mathrm{s}, \mathrm{Cu}$ lines were not observed with enough intensity to make temperature determination. The total number of data points that have been reduced to make the bottom panel is 40. Bottom: Temperature points before time-averaging. 
As was briefly touched upon in the previous section, the arc pulser induces noise in the MCT IR detector (see MCT pixel readout in Fig. 4.3) at early times relative to plasma development $(<3$ $\mu \mathrm{s}$ ) and is larger than the signal from the arrival of the laser pulse. However, even at later delay times noise still plagues the TRIR detector. For example, presented in Figure 4.9 is a comparison of the noise at two different delays ( 2 and $10.5 \mu \mathrm{s})$ relative to plasma development for pre-spark and transient spark. The noise assessment was made in the absence of laser light, i.e. the input to the IR spectrometer was blocked. This allowed us to make certain that the noise issue was independent of plasma development, e.g. absorption of the IR light by the plasma. What is clearly evident is that there is very little noise in the pre-shot data while the transient data contain a significant amount. At $2 \mu \mathrm{s}$, there is more noise in the transient than there is at $10.5 \mu \mathrm{s}$. This is consistent with the MCT readout shown in Fig. 4.3. Of major importance is that the signal is negative for the transients. Under normal experimental conditions, the MCT detector registers a positive voltage from laser irradiation. A smaller voltage usually translates into less transmission. For example, thicker PETN films attenuate the laser light more dramatically than thinner films thus leading to smaller voltages. Normally, a larger positive voltage would be observed in the absence of this induced noise because the film is ablating/reacting and becoming thinner (hence, more transmission or less absorbing) in response to the arc impulse. However, because the pulser is inducing a negative voltage bias into the detector during operation this makes the voltage less positive thereby indicating less transmission or more absorption than what is physically possible given post-shot analysis of the films, i.e. $\sim 300 \mathrm{~nm}$ of material is ablated/reacted. This induced noise is the likely reason that there is so much variation in the TRIR data (Fig. 4.8)
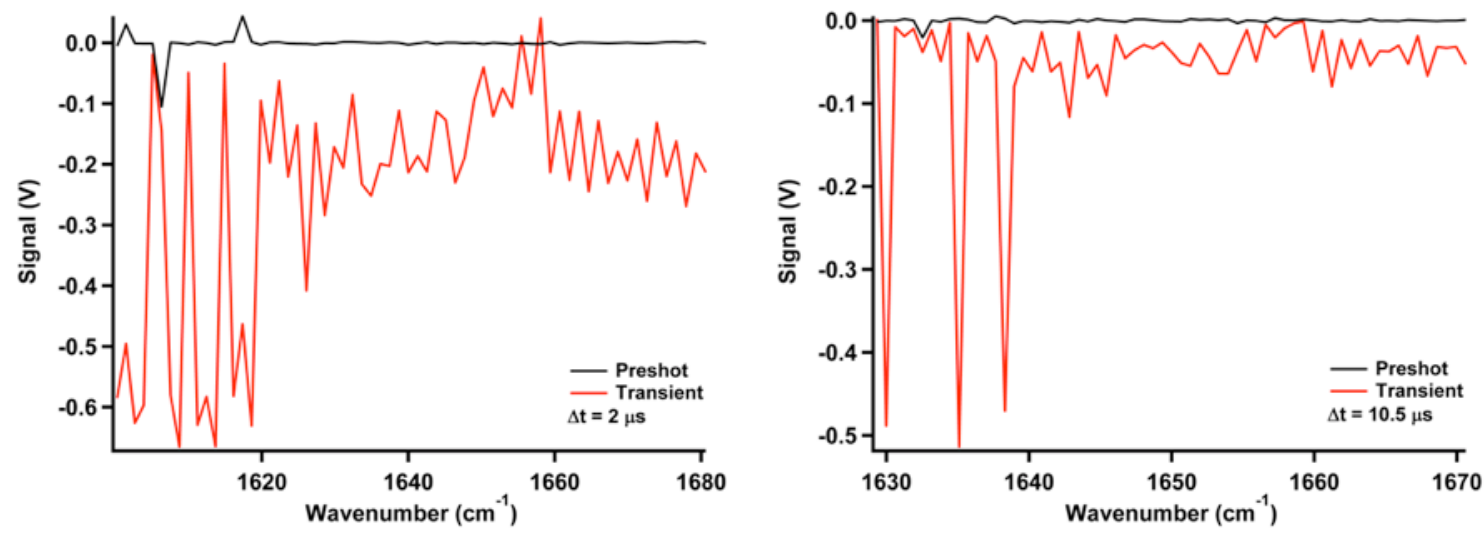

Figure 4.9. Comparison of noise intensity between pre- and transient signal at two different time delays in the MCT IR detector. At early delay time relative to plasma development the noise in the transient signal is much large than at later delay time. However, the noise level for both delay times when compared to the preshot signal is actually significant.

Several attempts were made to mitigate the noise issue. The solutions mostly involved shielding the IR detector or the pulser or trying to reduce ground loops or limiting cabling that could act as antennae. Shielding ultimately only had a minor effect, but was implemented nonetheless. In addition, we performed a careful analysis on some of the raw transmission data by varying the delay time as well as the detector gating while keeping the film thicknesses constant. Figure 
4.10 shows three different raw transmission spectra that consist of pre-, transient, and post-spark data. The top and middle panels are for a delay time of $2 \mu$ s but have 500 and $1000 \mathrm{~ns}$ IR detector gate widths, respectively. The bottom panel has a delay time of $5 \mu$ s and a gate width of 1000 ns. The $2 \mu$ s delays are "worst" case scenarios in terms of noise contamination from the arc pulser. First, there is more transmission post-spark than pre-spark, which is consistent with less material remaining after a spark insult. However, what is evident between both $2 \mu$ s cases is that the $1000 \mathrm{~ns}$ gate has more noise contamination from the pulser in the transiently sparked spectrum that manifests in the signal as actually reading a negative voltage, which is physically impossible as described earlier. When comparing the $500 \mathrm{~ns}$ gate, $2 \mu$ s delay to the $1000 \mathrm{~ns}$ gate, $5 \mu$ delay the magnitude of the noise is less with the 500 ns gate versus the $1000 \mathrm{~ns}$ gate where the $1000 \mathrm{~ns}$ spectrum shows a smaller positive voltage than the pre-spark spectrum. It should be pointed out that the 500 ns gate, $2 \mu$ s transient spectrum shows a similar transmission to the prespark, which is likely not possible. In any case, the conclusion that we drew from this more detailed analysis was that we should use a shorter gate width whenever possible to limit the noise pollution from the arc pulser.

Further, we also concluded that the PETN films were likely too thick. For a sample thickness of $\sim 1 \mu \mathrm{m}$, the optical density was approximately 2 . This translates approximately to a transmission of $\sim 1 \%$. The voltage that corresponds to a transmission of $1 \%$ is on the order $100 \mathrm{mV}$. A negative voltage of that order can be induced by the pulser (see Figure 4.9). To mitigate any negative voltages in the raw data, thinner HE films of approximately $500 \mathrm{~nm}$ were spun coated. 

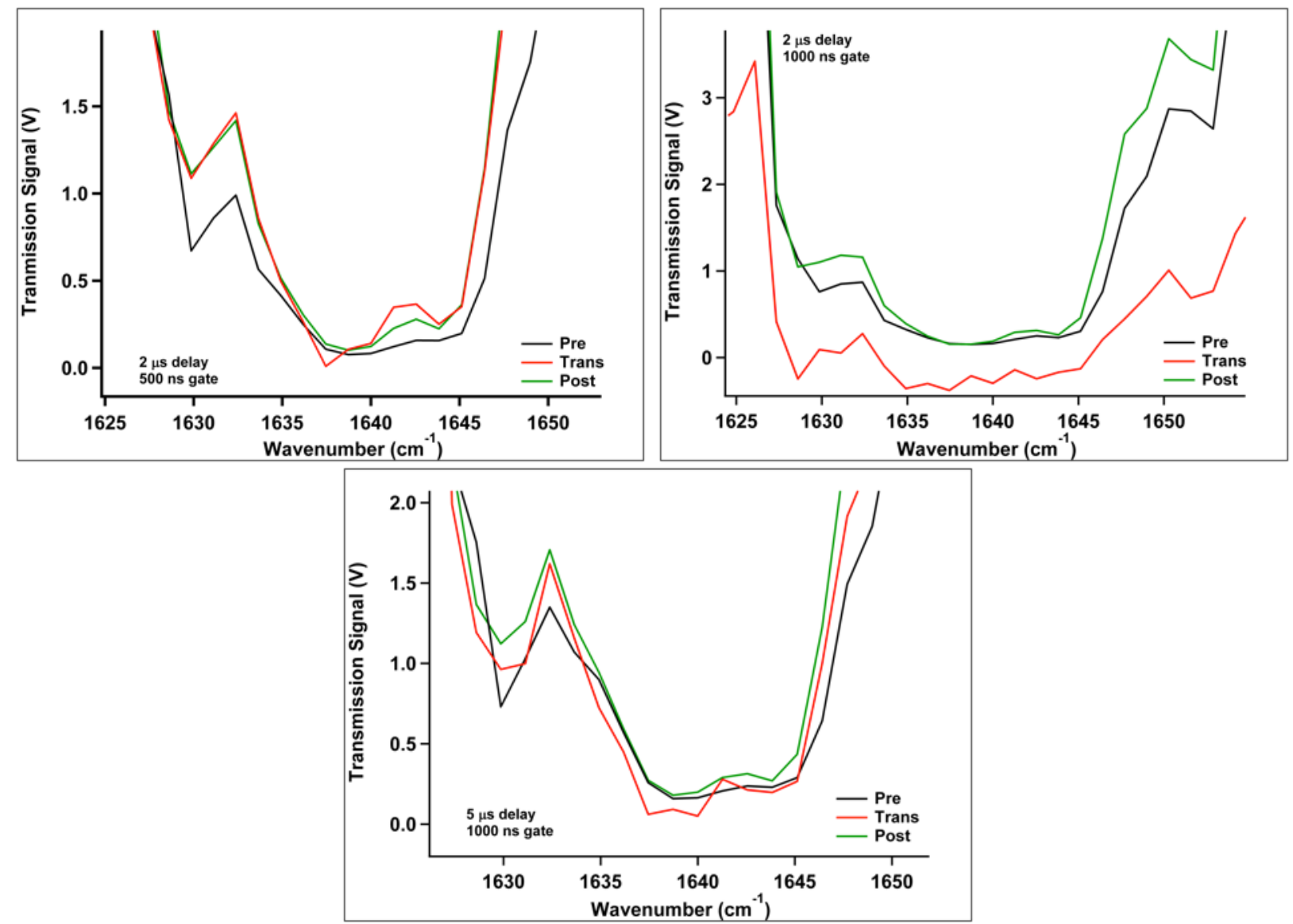

Figure 4.10. Three different raw transmission spectra that consist of pre-, transient, and post-spark data for a PETN thin film of approximately $1 \mu \mathrm{m}$.

\subsection{Hardware failure and attempted recovery}

On 4/13/11, there was a catastrophic failure of the initial pump laser that drives the fs IR system. Initially the diagnosis was that the diodes on the Verdi laser failed. However, after replacing the diodes it was determined that the laser head was the actual component that had failed. All in all, it took more than 8 weeks from initial failure to final repair on 6/16/11 thus jeopardizing finishing the TRIR work by the end of the LDRD project. This 8-week downtime from the failure of the Verdi laser was compounded by other downstream laser problems. We have noticed in the past that when the fs mid-IR system sits idle on the order of weeks to months the optical alignment changes detrimentally. Hence, the OPA/DFG system that generated the midIR pulses was not operational after repair of the Verdi laser and needed to be realigned.

We encountered several additional problems during realignment. We noticed soon after sufficient alignment to generate IR pulses was accomplished that the energy/pulse was low. After careful examination, it was determined that, for reasons unknown, the pump beam from the fs amplifier was focusing in the OPA/DFG. This had the effect of burning several mirrors in the OPA and DFG that lead to the decline in power. These mirrors were replaced. Further, the beam qualities in the OPA over the various optical paths were poor and had to be repaired and required a general realignment of the entire OPA/DFG system from first-principles. All of these 
additional problems took approximately another month to remedy to the point that new data could be collected.

Several data points were collected and Figure 4.11 shows a sample TRIR spectrum for one time delay. The transient data illustrates the continuing noise issue that has plagued all TRIR measurements. The spikes that are evident in the transient data (and also in the transient difference spectra) make the data unusable and were evident in nearly all data taken in the last TRIR campaign. Even using thinner films combined with a shorter gate width ( $500 \mathrm{~ns})$ as was determined from the $2^{\text {nd }}$ TRIR campaign did not fully mitigate the noise issue.

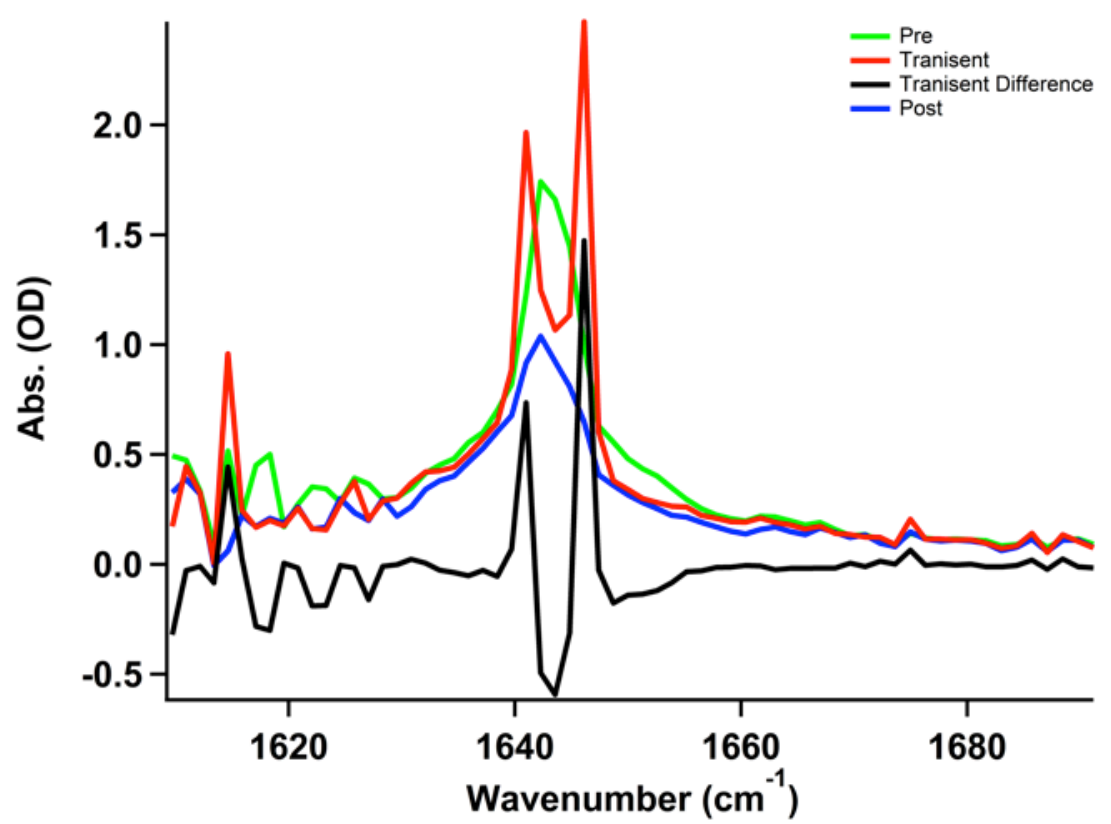

Figure 4.11. Pre-, transient, post-, and transient difference TRIR spectra for a time delay of $10 \mu$ from the development of the plasma. 


\section{Pumped Convective Burn}

NOTE: The first few subsections (5.1-5.5) were also included in CODT-2010-1816, an internal annual report for NSR\&D activities, because it was also of interest to that community.

In this section, we will present a model referred to as "pumped convective burn" (PCB), which shows promising signs of reproducing observed ESD threshold behavior. We emphasize that this work is still in its early stages, and either data acquired in the near future or corrections to the model and its numerical implementation could quickly invalidate it.

This section is rather long and so we provide a breakdown of the subsections in it. Subsection 5.1 gives background on the observations and assumptions that drive the model. Subsection 5.2 gives a physical picture of the process and subsection 5.3 presents a mathematical model for it. Subsection 5.4 discusses the choices for a number of physical parameters in the model, and specifically motivates the values chosen for the numerical study described in subsection 5.5. Subsection 5.6 further pursues the important topic of the burn rate of the HE grains, from a temperature-based perspective. Subsection 5.7 presents the derivation of a steady PCB wave, which can release energy from the HE on fast time scales and short length scales without requiring shock compression of the solid-phase HE (as would occur in a detonation) or compaction of low-density material (as would occur in a deflagration-to-detonation transition, or DDT). We believe this is the first such derivation of this type of wave, which may be important in our understanding of low-density HE initiation and the general response of HE to fast thermal insults. Subsection 5.8 summarizes the steady-wave work, and 5.9 lists the references particular to this section.

\section{$\underline{\text { 5.1. Background }}$}

The focus of this LDRD was intended to be on the high-temperature kinetics of arc-driven HE, and therefore the experimental configuration is not directly pertinent to pin-to-case discharges in macroscopic ( $\sim 1$ gram as opposed to $<10 \mathrm{mg}$ ) HE samples; however, two particular results from the LDRD characterization work have provided what may be key insights into the initiation of detonator-size quantities of low-density HE. The test configuration referred to in the bullets below is shown in Figure 5.1.1.

- The discharge in the LDRD experiments is between two thin electrodes pressed against a thin film of solid PETN. The properties of the discharge are very repeatable and it is observed to occur in the air above film, not through the film itself.

- Only a fraction of the film's thickness is reacted or at least vaporized as the discharge evolves hydrodynamically against the surface; the volume of material that is removed is quantitatively consistent with what would be expected from rapid ablation (see the reference for a bit more detail). In other words, an initially heated and vaporized volume is quickly convected away from the free surface before any other transport mechanism can heat more of the interior volume of the film. 


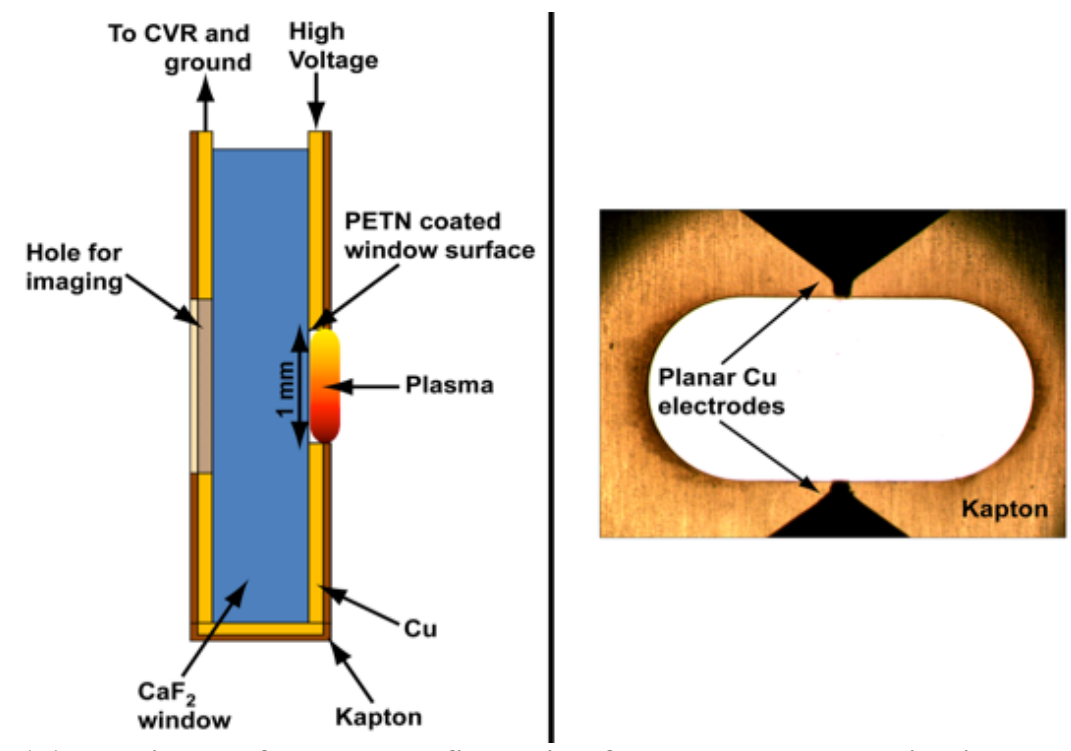

Figure 5.1.1. Reminder of the test configuration for the arc characterization experiments.

In a pin-to-case discharge in a detonator configuration, the discharge is either at an interface between a solid surface and a low-density pressing of HE, or through the low-density volume itself. By "low density" we mean about half of theoretical maximum density (TMD) of the HE in question. In other words, half of the region in question is in fact air-filled void space. Thus, we map the two observations above to the following two assumptions about a macroscopic lowdensity volume:

- The discharge occurs through the air in the voids, not through the solid HE grains. This means that the resistive heat from the discharge is not directly heating solid HE.

- The subsequent energy transport during and after the discharge is convection dominated. Flow through the voids will be an influence on behavior that is at least comparable if not greater than transverse interactions between the heated voids and the grains.

Even if we temporarily ignore the energetic behavior of the HE, the two points above imply a system whose detailed behavior is taking place on the very small and geometrically complex scale of individual HE grains. To maintain tractability, we would nevertheless like to work with a continuum model. In order to do so, certain constraints must be satisfied:

- By the first assumption, the fluid in the voids and the HE grains are not in thermal equilibrium. A continuum model must follow two distinct temperature fields.

- To model the behavior described by the second assumption, we will bring to bear some of the machinery developed in the field of flow in porous media. While this is a heavily studied area, to this day it remains true that tractable porous media models must invoke some empirical ingredients. We will discuss this in more detail in the next section. 


\subsection{Physical Model}

Let us introduce some relevant time and length scales. For simplicity, material properties are assumed to be constants.

We characterize the input electrical source as a roughly cylindrical discharge with an initial radial size scale of $\mathrm{r}_{\mathrm{s}} \sim 0.125 \mathrm{~mm}$ and a length $\mathrm{L}_{\mathrm{s}}$ of $2.5 \mathrm{~mm}$. It deposits a total energy $\mathrm{E}_{\mathrm{s}} \sim 100$ $\mathrm{mJ}$ over a time $\mathrm{t}_{\mathrm{s}} \sim 100 \mathrm{~ns}$.

The HE grains have a size scale $\mathrm{r}_{\mathrm{HE}} \sim 10 \mathrm{~mm}$, a solid density $\rho_{\mathrm{HE}} \sim 1800 \mathrm{~kg} / \mathrm{m}^{3}$, a specific heat $\mathrm{C}_{\mathrm{HE}} \sim 1100 \mathrm{~J} / \mathrm{kg}-\mathrm{K}$, and a thermal conductivity $\kappa_{\mathrm{HE}} \sim 0.25 \mathrm{~W} / \mathrm{m}-\mathrm{K}$. They are also characterized by a specific surface area $\mathrm{S}_{\mathrm{HE}} \sim 700 \mathrm{~m} / \mathrm{kg}$, which is considerably higher than the spherical value of $3 / \rho_{\mathrm{HE}} \mathrm{r}_{\mathrm{HE}}=167 \mathrm{~m} / \mathrm{kg}$; the grains are quite irregular in shape. They are initially at room temperature, $T_{i}=300 \mathrm{~K}$. The only information about its energetic nature that we invoke at this stage is that a self-sustaining detonation wave would travel through bulk solid material at a speed $\mathrm{c}_{\mathrm{HE}} \sim 7 \mathrm{~mm} / \mathrm{ms}$.

The void space is initially filled with an ideal stand-in for air, with initial density $\rho_{\mathrm{i}}=1.2 \mathrm{~kg} / \mathrm{m}^{3}$ and in thermal equilibrium with the HE at temperature $T_{i}$. The molecular weight is assumed to be 28 and the equation of state is that of an ideal low-temperature diatomic gas with ratio of specific heats $\gamma=7 / 5$. For purposes of estimating a Reynolds number, we use a viscosity $\mu$ of $1.8 \times 10^{-5} \mathrm{~kg} / \mathrm{m}-\mathrm{s}$.

The overall macroscopic volume of the system has a length scale $\mathrm{L} \sim 1 \mathrm{~cm}$.

We now have enough information to begin drawing some interesting conclusions about the system behavior. For grains packed into the volume at half of the bulk density, the characteristic void diameter $\mathrm{d}$ will be on the order of the grain diameter, $\sim 20 \mu \mathrm{m} ; \mathrm{d} / \mathrm{L}<<1$ and so a continuum porous media model should be feasible.

On the timescale of the input pulse, the characteristic thermal diffusion length into the HE (if it were to remain inert) is:

$$
l_{\text {conduct }} \sim \sqrt{\frac{\kappa_{H E} t_{s}}{\rho_{H E} C_{H E}}} \sim 0.1 \mu \mathrm{m}
$$

and so $1_{\text {conduct }} / r_{\mathrm{HE}}<<1$. Thus, not only are the fluid in the void and the HE not going to remain in thermal equilibrium, but there will be significant temperature gradients within an individual grain.

Conductive heat transport through the grains but parallel to the flow direction has a different length scale that is imposed by the scale of the problem - it is L, the system scale. Even if we assume the grains were a continuous medium, the thermal diffusion time is quite long:

$$
t_{\text {parallel }} \sim \rho_{H E} C_{H E} L^{2} / \kappa_{H E} \sim 1000 s
$$


Thus, along the flow direction, the dominant coupling mechanism grain to grain is via the convective heat transfer to and from the fluid. Thermal conduction parallel to the flow can be ignored.

If we take the extreme case where all of the input energy goes into the air with no convection, conduction, or other losses, the peak pressure would be:

$$
P \sim \frac{2(\gamma-1) E_{s}}{\pi r_{s}^{2} L_{s}} \sim 6.5 \times 10^{8} \mathrm{~Pa}
$$

and in actual model results will tend to be less than $10^{8} \mathrm{~Pa}$, or an order of magnitude too low to be relevant for shock initiation over the run distance $\mathrm{L}$ and certainly far too low for shock-based detonation of individual grains in the early stages of the initiation process. Instead, the individual grains are initially consumed via a conductive burn wave, much slower than a shock wave. However, we posit that in the regime of interest the wave speed $\mathrm{v}_{\text {burn }} \sim 10 \mathrm{~m} / \mathrm{s}$, significantly faster than what would be encountered in an unconfined, slow "cookoff" type of experiment. Such a burn wave would consume an individual grain in a time $\mathrm{r}_{\mathrm{HE}} / \mathrm{v}_{\text {burn }} \sim 1 \mathrm{~ms}$.

Finally, given $\mathrm{P} / \mathrm{P}_{\mathrm{i}}>>1$ where $\mathrm{P}_{\mathrm{i}}$ is the initial (ambient) pressure, we expect that the peak fluid velocity $\mathrm{v}$ will be on the order of the sound speed $\mathrm{c}$ :

$$
v \sim c \sim \sqrt{\frac{\gamma P}{\rho}} \sim 10 \mathrm{~mm} / \mu \mathrm{s}
$$

Thus, even though the flow is through a porous media, it will remain compressible. In spite of the small void size, the high velocity gives a Reynolds number $\operatorname{Re}=\rho \mathrm{vd} / \mu \sim 10^{4}$ and the flow will also be turbulent. Turbulence-enhanced heat transfer and shear viscosity must be taken into account. Enhancement of normal viscosity will also occur but not to the extent of smearing out normal gradients enough to avoid numerical treatment of shocks. Whether such shock fronts would remain stable in the porous context is beyond the authors' current level of understanding.

If the fluid velocity were sustained at these levels, then observe that the transit time through the detonator volume, $t_{\text {trans }}=\mathrm{L} / \mathrm{v}$, is $\sim 1 \mu$ s and is of the same order as the burn time for an individual grain. Thus a possible physical picture of arc initiation begins to emerge: a high-velocity flow of high temperature fluid in the void space ignites the surface of the HE grains throughout the entire volume in a short time. Then a thermal burn wave rapidly releases energy from the grains. Thus, energy is released from the entire volume within a few microseconds, even though a traditional shock-based detonation never takes place. Grain compaction, which plays a fundamental role in deflagration-to-detonation transition (DDT) in larger samples (typically tens of $\mathrm{cm}$ are studied in experiments) [9; 7, Ch. 8], may occur but is not required and in fact will be neglected in what follows (really without much justification at this point).

We refer to this picture as "pumped convective burn" because the high input power from the arc bootstraps the process, and the high temperatures achieved can sustain a faster than typical thermal burn wave which in turn continues to drive the convective flow in a self-sustaining pumping process. The pumped convective burn regime is characterized by the two properties: 


$$
\begin{aligned}
& t_{\text {trans }} \sim 1 \mu s \\
& \frac{L v_{\text {burn }}}{r_{H E} v} \sim 1
\end{aligned}
$$

which must be justified in what follows. Note that what is achieved in this regime is not a detonation in the strict sense, but may be more than sufficient to drive (for example) an output pellet in a detonator in the intended mode.

\subsection{Mathematical Model}

The mathematical model used to study pumped convective burn is based on one used with much success on a problem in a completely different area but which has many similarities: propagation of a quench front in cable-in-conduit type superconductors (CICCs). CICCs consist of bundles of superconducting strands with copper cladding, packed into a conduit. The strands are kept in the superconducting state by flowing cryogenic helium through the conduit. If an off-normal occurrence causes a local region of the strands to heat and transition to a resistive phase, the current transfers from the (no longer) superconducting material in the core of the strands to the copper cladding; however, the current density in the copper and the resistivity of the copper are still sufficiently high that rapid Joule heating occurs in the strands and the heat is quickly transferred to the surrounding helium. The hot helium then convects to a larger region, heating an increasing volume of the cable in a runaway event called a "quench." The tight packing of the strands in the conduit produces a porous-media type of flow environment, with a characteristic void dimension on the order of $1 \mathrm{~mm}$ in cables that are tens of meters in overall length.

Details of a successful thermal hydraulic model of CICCs can be found in a series of papers by Shajii and Freidberg $[1,2,3]$ and the doctoral thesis of A. Shajii, available from the MIT Plasma Science and Fusion Center [4]. This model will be adopted here without repeating the full derivation, and with some important modifications:

- In a quench, once the Joule heating source turns on, it remains on for a long time scale (in actual operation, the electrical current is dumped from the cable when a quench is detected, but this takes time). In pumped convective burn, the heating from burning HE is transient and an appropriate reactive model must be included.

- CICC quench falls into a unique flow regime called "low Mach number compressible flow," which as the name implies means the flow retains significantly compressibility even though the flow velocity remains highly subsonic. This occurs because frictional forces from the small void diameter can, over tens of meters of flow length, support enough of a pressure gradient to dominate inertial effects. This is not the case in pumped convective burn and the inertial terms in the model must be retained.

The turbulent correlations used in CICC modeling for heat transfer and friction from viscous shear are adopted in our pumped convective burn model without rigorous investigation into their applicability. Given that such empirical correlations are highly dependent on surface characteristics and geometric details, it is likely that these choices are not appropriate and this will have to be addressed in future work. 
The model treats a one-dimensional cylindrically symmetric flow geometry, compounded with a locally transverse direction "into" the HE grains. The radial coordinate $r$ denotes distance from the center of the original electrostatic discharge; the ratio of discharge radius to length is considered small enough that we neglect axial dependence. The coordinate $\mathrm{x}$ denotes distance from the center of any given HE grain. For now, the grains are treated as uniform in size and shape, with the actual complex shape represented by using a ratio of wetted perimeter to flow area that is weighted by the measured specific surface area (more detail will be given later). Figure 5.3.1 shows a somewhat more realistic picture of the physical situation, while Figure 5.3.2 shows that the mathematical equivalent resembles a cylindrical flow (through the pores) between closely spaced, uniform radial slabs (represented the HE grains).

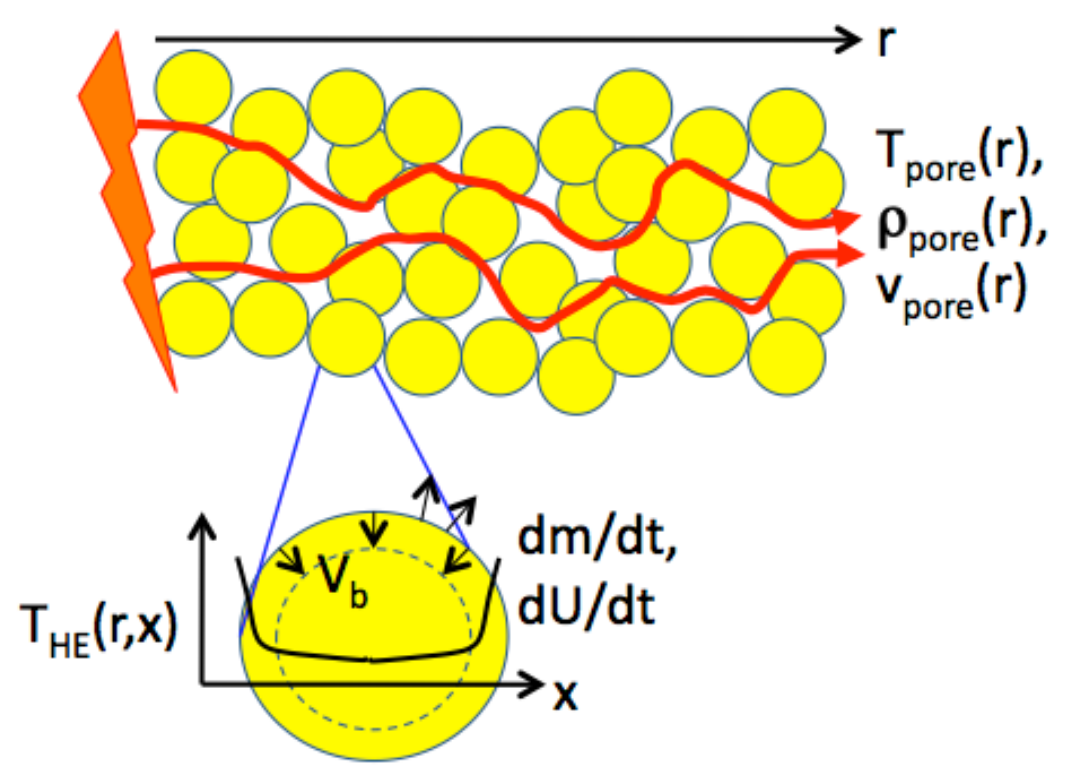

Figure 5.3.1. Schematic of flow through the pores and temperature gradient across individual grains.
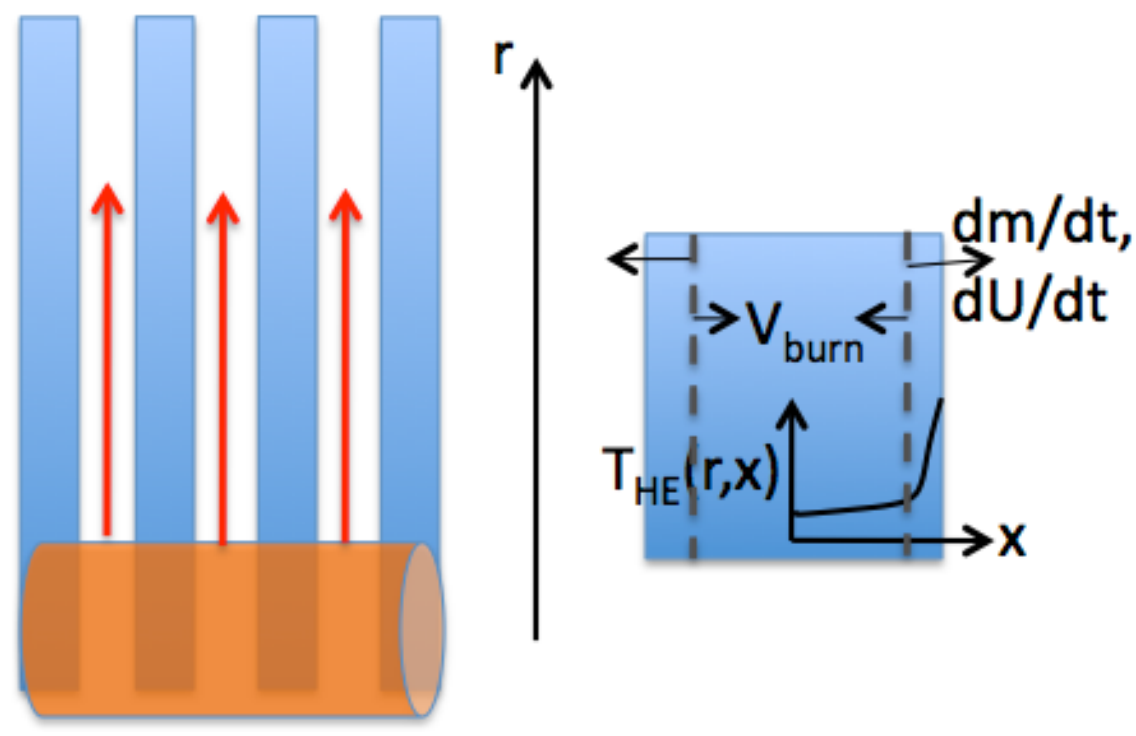

Figure 5.3.2. Schematic of the system as represented by the mathematical model. Note that there are significant gradients across the HE (now represented as slabs) but not across the channels representing the pores. 
The system of equations consists of conservation of mass, momentum, and energy in the fluid and energy conservation in the grains. A reaction model for the grains must also be included but is not written explicitly below as a variety of models can be used; specific examples will be presented later.

The notation:

$\begin{array}{ll}r & \text { radial distance from the center of a cylindrically symmetric arc } \\ \mathrm{t} & \text { time } \\ \mathrm{x} & \text { local coordinate across the HE grains } \\ \rho & \text { fluid density } \\ \mathrm{v} & \text { fluid velocity } \\ \mathrm{P} & \text { fluid pressure } \\ \mathrm{T} & \text { fluid temperature } \\ \mathrm{U} & \text { fluid internal energy per unit volume (not specific!) } \\ \mathrm{E} & \text { total fluid energy per unit volume; } \mathrm{E}=\mathrm{U}+\mathrm{pv}^{2} / 2 \\ \mathrm{~m} & \text { average molecular mass of the fluid } \\ \mathrm{k}_{\mathrm{B}} & \text { Boltzmann's constant } \\ \gamma & \text { ratio of specific heats in ideal EOS } \\ \mathrm{d} & \text { hydraulic diameter of the pores } \\ \mathrm{h} & \text { heat transfer coefficient (usually turbulent) between the fluid and HE } \\ \mathrm{f} & \text { friction factor (usually turbulent) to represent viscous shear in the pores } \\ \mathrm{S} & \text { production rate of reaction products entering the flow in the pores } \\ \mathrm{Q}_{\mathrm{ext}} & \text { input heating term from the arc } \\ \mathrm{P}_{\mathrm{w}}, \mathrm{A}_{\mathrm{w}} & \text { wetted perimeter and area, respectively; this is an accounting of the irregular } \\ & \text { grain shape } \\ \mathrm{S}_{\mathrm{HE}} & \text { specific surface area of the HE grains } \\ \rho_{\mathrm{HE}} & \text { density of solid HE (the density of an individual grain; not a packing density) } \\ \mathrm{C} & \text { specific heat of solid HE } \\ \mathrm{T}_{\mathrm{HE}} & \text { HE temperature } \\ \mathrm{Q}_{\mathrm{HE}} & \text { energy release in the reacting HE } \\ \mathrm{U}_{\mathrm{HE}} & \text { internal energy of reacted material as it flows into the pores from the grains } \\ \kappa_{\mathrm{HE}} & \text { thermal conductivity of the HE } \\ \mathrm{f}_{\mathrm{HE}} & \text { fraction of HE still in the unreacted state }\end{array}$

The system of equations is:

$$
\begin{aligned}
& \frac{\partial \rho}{\partial t}+\frac{1}{r} \frac{\partial}{\partial r}(r \rho v)=\rho_{H E} S\left(f_{H E}, t\right) \\
& \frac{\partial \rho v}{\partial t}+\frac{1}{r} \frac{\partial}{\partial r}\left(r \rho v^{2}\right)=-\frac{\partial P}{\partial r}-\frac{f \rho v|v|}{2 d} \\
& \frac{\partial E}{\partial t}+\frac{1}{r} \frac{\partial}{\partial r}(r v(E+P))=Q_{e x t}(r, t)+U_{H E} S\left(f_{H E}, t\right)-\frac{h P_{w}}{A_{w}}\left(T-T_{H E}\right)+\frac{f \rho v^{2}|v|}{2 d}
\end{aligned}
$$




$$
\begin{gathered}
\rho_{H E} C \frac{\partial T_{H E}}{\partial t}=\kappa_{H E} \frac{\partial^{2} T_{H E}}{\partial x^{2}}+Q_{H E}(r, x, t) \\
\text { subject to } \quad-\left.\kappa_{H E} \frac{\partial T_{H E}}{\partial x}\right|_{e d g e}=h\left(T-T_{H E}\right) \\
\text { and } \quad-\left.\kappa_{H E} \frac{\partial T_{H E}}{\partial x}\right|_{0}=0 \quad \text { (symmetry at the grain center) } \\
\frac{\partial f_{H E}}{\partial t}=\ldots \quad \text { (various kinetic models) }
\end{gathered}
$$

Finally, the system is closed by the definition:

$$
E=U+\frac{1}{2} \rho v^{2}
$$

and the ideal state equations

$$
P=(\gamma-1) U \quad \text { and } \quad T=\frac{(\gamma-1) m}{k_{B}} U / \rho
$$

\subsection{Various Empirical Choices}

In this section we present the choices made for specific values or models used to calculate some parameters in the above system.

\subsubsection{Heat Transfer and Viscous Shear}

It is very instructive to explore the effects of heat transfer and viscous drag by doing parametric studies across various constant values of $h$ and $f$. However, since in many cases the flow does not fully develop until the input energy from the arc has been turned off, a more realistic bootstrapping of pumped convective burn should take into account the transition from lower (more laminar) to higher (turbulent) flow velocity. As mentioned above, we have explored the effect of some empirical correlations for $h$ and $f$, albeit without much study on whether the chosen correlations are the most appropriate.

For the heat transfer coefficient, we use the same approach as in the references. Defining the heat transfer coefficient in terms of the Nusselt number, $\mathrm{N}$, fluid thermal conductivity $\kappa$, and hydraulic diameter $\mathrm{d}$ :

$$
h \equiv \frac{\kappa N}{d}
$$

For laminar flow, we use $\mathrm{N}=\mathrm{N}_{1}=4$. For turbulent flow, $\mathrm{N}=\mathrm{N}_{\mathrm{t}}$, we use the Dittus-Boelter correlation but keep the Prandtl number Pr fixed at 1:

$$
N_{t}=0.026 \operatorname{Re}^{0.8} \operatorname{Pr}^{0.4}\left(T / T_{H E}\right)^{0.716}
$$


where Re denotes the Reynolds number, $\rho v d / \mu$. To create a single, continuous function, we blend the two so that they transition at about $\mathrm{Re}=5000$ :

$$
N=N_{l}+N_{t} e^{-(5000 / R e)^{2}}
$$

The friction factor is usually held constant at 0.01 , but when a Reynolds number dependent form is desired we again take directly from the references,

$$
f=0.184 / R e^{0.2}
$$

where, due to lack of any real data for this configuration, we have dropped a leading scaling coefficient of order unity which attempts to account for surface roughness.

The meaning of $\mathrm{P}_{\mathrm{w}} / \mathrm{A}_{\mathrm{w}}$ is another open question. Because of the continuous nature of the strands in CICCs, the wetted perimeter and area are well-defined quantities. In the packed-powder geometry, it is apparent that a re-derivation of the approximation of turbulent heat transfer should be performed and we have not (yet) done so. Two possible choices of force-fitting this factor in the current model are to use the ratio of the surface area to the grain volume, or the square-root of the surface area to the grain cross-sectional area:

$$
P_{w} / A_{w} \sim \rho_{H E} s_{H E} \quad \text { or } \quad \sim \sqrt{4 \rho_{H E} s_{H E} / 3 \pi r_{H E}}
$$

Because the magnitude of the heat transfer is important to reaching the pumped convective burn regime, we do not want to artificially boost the heat transfer too much and perhaps deceive ourselves into the existence of the pumping mechanism. Thus, until the matter can be investigated with more rigor, we choose the square-root form. (Writing $\mathrm{S}_{\mathrm{HE}}$ in the form of a multiplicative factor over the spherical value shows that, for the typical measured values of $\mathrm{s}_{\mathrm{HE}}$, the square-root form is indeed smaller.)

\subsubsection{External Heating}

Framing camera images of the discharge between the electrodes show that the initial streamer is very well defined by the electrode geometry-i.e., there is a single streamer spanning the gap, whose diameter matches the electrode width. In threshold testing experiments performed under the Nuclear Safety R\&D portfolio, whose thresholds are what we ultimately want to predict, the discharge is between two 10-mil (about 250 microns) wide copper electrodes separated by a gap of 100 mils (about $2.5 \mathrm{~mm}$ ). In the model we wish to work with a smooth distribution and so we represent the source as having a Gaussian profile in $r$, with a characteristic radius of 125 microns. The total source energy is in the range $10-100 \mathrm{~mJ}$ and thus the $2.5 \mathrm{~mm}$ arc length in the NSR\&D configuration specifies the input energy density. The temporal profile is taken to be constant over a specified pulse length, typically $100 \mathrm{~ns}$; this specifies the input power density.

Data from the experiments shows that the arc does evolve hydrodynamically while energy is still being input; we do not yet take this into account. In other words, even though the model does convect the heated fluid during the pulse, the heating is still deposited over a fixed profile in the Eulerian frame. 
As stated earlier, the initial fluid in the pores is idealized air, at a density of $1.2 \mathrm{~kg} / \mathrm{m}^{3}$ and temperature of $300 \mathrm{~K}$, with a corresponding initial pressure close to $1 \mathrm{~atm}$. The molecular weight $\mathrm{m}$ is taken to be 28 and the ratio of specific heats $\mathrm{g}$ to be $7 / 5$. When required to evaluate $\mathrm{h}$ and $\mathrm{f}$, the thermal conductivity is taken to be $0.024 \mathrm{~W} / \mathrm{m}-\mathrm{K}$ and the viscosity is $1.8 \times 10^{-5} \mathrm{~kg} / \mathrm{m}$ S.

\subsubsection{Burn Model}

The details of the grain-level burn model are critical to pumped convective burn, because the speed at which an HE grain is consumed by a conductive burn wave is a key contributor to the overall physics. There are numerous models in the literature $[5,6]$ but none of those examined thus far meet the correct requirements. Some features that typically result in a mismatch include lack of reactant depletion, an assumed high activation energy limit, or boundary conditions for the flow that do not apply to our conditions (for example, a constant external pressure, with negligible convective effects, is often used).

At this time, we have not developed what we feel to be a fully consistent model. We will describe some simple cases that are easy to implement and then present two limiting calculations that show how the burn rate can be very sensitive to model assumptions.

The speed of the burn wave in a grain is the desired output of the burn model. If the average molecular weight of the fully reacted products is not too different from air, then even at a relatively low product temperature of $1000 \mathrm{~K}$ we get a thermal speed of about $500 \mathrm{~m} / \mathrm{s}$. The corresponding transverse transit time (across the void space) for a 10-micron void is about $20 \mathrm{~ns}$, fast compared to the overall timescales of interest (hundreds of ns to microseconds). Therefore, we convert the transverse flux of mass and energy to a volumetric source within the voids by means of the source rate $S=2 v_{\text {burn }}(r, t) / d$, where the factor of two appears because opposing faces of grains will be contributing to the same void.

In the simplest test case, we use a simple threshold model to represent the burning of the HE. While the grain surface is below a critical temperature, the grain is inert. Above that temperature, $\mathrm{v}_{\text {burn }}$ is taken to be constant until a time $\mathrm{r}_{\mathrm{HE}} / \mathrm{v}_{\text {burn }}$ has past, at which point the grain is considered consumed. The parameter $\mathrm{v}_{\text {burn }}$ is a knob but we tend to focus on a value of about 10 $\mathrm{m} / \mathrm{s}$, at which level interesting behavior is observed.

The HE we try to represent is PETN. We use a solid density of $1800 \mathrm{~kg} / \mathrm{m}^{3}$, a specific heat of $1100 \mathrm{~J} / \mathrm{kg}-\mathrm{K}$, and a thermal conductivity of $0.25 \mathrm{~W} / \mathrm{m}-\mathrm{K}$. For the threshold model, we assume that the reaction rate has become fast relative to any other timescale by a temperature of $1000 \mathrm{~K}$. For a single-step Arrhenius model, we use parameters similar to those of the final step of the four-step Tarver/Tran/Whipple model [8], with a rate constant of $1 . e 14 \mathrm{~s}^{-1}$ and an activation energy of $145 \mathrm{~kJ} / \mathrm{mol}$ (somewhat higher than the fourth-step value, but lower than earlier steps) which maps to a temperature of $17400 \mathrm{~K}(1.5 \mathrm{eV})$. The net energy release is taken to be 6 $\mathrm{MJ} / \mathrm{kg}$, meaning the model parameter $\mathrm{U}_{\mathrm{HE}}$ is given by $\rho_{\mathrm{HE}}\left(\mathrm{CT}_{\mathrm{i}}+6 \mathrm{MJ} / \mathrm{kg}\right)$. Ideally, we would like to incorporate a full multi-step model, including endothermic steps; we expect the latter to have a significant effect, particularly when looking ahead to studying highly insensitive TATB. 


\subsubsection{Hard-threshold Limit}

Let us consider the hard-threshold model, where the HE is inert below some critical temperature $\mathrm{T}_{\text {crit, }}$, then reacts instantly above that threshold. We assume that the reacted material is mixed into the void space on a fast time scale and is the dominant contribution to the volumetric energy density in the voids. We further assume that variation of the temperature of the reacted fluid in the void, as well as acceleration of that fluid, occurs on a slow time scale. Thus we expect the fluid in the void to act as a thermal reservoir at a temperature of order $\mathrm{U}_{\mathrm{HE}} / \rho_{\mathrm{HE}} \mathrm{C}$. This is not a great assumption since the total thermal mass in the void space will in general be less than that of the grains and thus heat loss into the burning grain is not negligible. However, the idealized limit we are taking will already produce a result that does not attain the burn velocity required to support the pumping mechanism.

We slightly modify the coordinate convention used previously. Consider a steady burn wave moving in the positive $\mathrm{x}$ direction, which is now taken to be moving into a grain. The wave position is defined to be $x=0$ in a frame moving at $\mathrm{v}_{\text {burn }}$. Ahead of the wave, the HE is below $\mathrm{T}_{\text {crit }}$ and therefore inert. Energy is transported into the inert material via thermal conduction. Far ahead of the wave, the grain is assumed to be at its initial, ambient value $T_{i}$. Behind the wave, we assume heat transfer is taking place from the hot, fully reacted fluid filling the void space; the fluid is at some constant, specified temperature $T_{\text {fluid }}>T_{\text {crit. }}$.

The resulting model is a single ODE which is the steady-state heat conduction equation in the moving frame:

$$
-v_{\text {burn }} \frac{d T}{d x}-\frac{\kappa_{H E}}{\rho_{H E} C} \frac{d^{2} T}{d x^{2}}=0
$$

subject to three boundary conditions:

$$
\begin{aligned}
& \left.T\right|_{x=0}=T_{\text {crit }} \\
& -\left.\kappa_{H E} \frac{d T}{d x}\right|_{x=0}=h\left(T_{\text {ext }}-T\right)=h\left(T_{\text {ext }}-T_{\text {crit }}\right) \\
& \left.T\right|_{x \rightarrow \infty}=T_{i}
\end{aligned}
$$

The appearance of three conditions rather than two is what provides an eigenvalue condition to determine $\mathrm{v}_{\text {burn. }}$. Integrating the ODE from 0 to $\mathrm{x}$ and applying the initial conditions, we get (using a prime to denote differentiation and dropping the "burn" subscript):

$$
T^{\prime}+\frac{\rho_{H E} C v}{\kappa_{H E}} T=\left.T^{\prime}\right|_{0}+\left.\frac{\rho_{H E} C v}{\kappa_{H E}} T\right|_{0}=\frac{h}{\kappa_{H E}}\left(T_{c r i t}-T_{e x t}\right)+\frac{\rho_{H E} C v}{\kappa_{H E}} T_{c r i t}
$$

This is easily solved:

$$
T(x)=T_{c r i t} e^{-\rho_{H E} C v x / \kappa_{H E}}+\frac{\left(h / \kappa_{H E}+\rho_{H E} C v / \kappa_{H E}\right) T_{c r i t}-h T_{e x t} / \kappa_{H E}}{\rho_{H E} C v / \kappa_{H E}}
$$


Enforcing the third boundary condition, ahead of the front, means:

$$
T_{i}=\frac{\left(h / \kappa_{H E}+\rho_{H E} C v / \kappa_{H E}\right) T_{c r i t}-h T_{e x t} / \kappa_{H E}}{\rho_{H E} C v / \kappa_{H E}}
$$

which can only be true if:

$$
v_{\text {burn }}=\frac{\frac{h}{\rho_{H E} C}\left(T_{\text {ext }} / T_{\text {crit }}-1\right)}{1-T_{i} / T_{\text {crit }}}
$$

For a rather extreme case of $T_{\text {ext }} / T_{\text {crit }} \sim 5$ and assuming $T_{i} / T_{\text {crit }}<<1$ gives:

$$
v_{\text {burn }} \approx 0.006 N \text { in } \mathrm{m} / \mathrm{s}
$$

where $\mathrm{N}$ is the Nusselt number and we have assumed a hydraulic diameter of 10 microns and that the thermal conductivity of the reacted-product fluid is the same as air. The latter assumption is weak but a correction to it (which would likely move in a favorable upwards direction) is not useful since the Nusselt number is not likely to be very high; for moderate temperature ratios, the Dittus-Boelter value for $\mathrm{N}$ does not reach $\sim 100$ until $\operatorname{Re} \sim 10^{4}$ (in fact, that correlation is not valid much below that range of Re). $\mathrm{N}$ is of course clamped on the low end by the laminar limit of 4 . Thumbnailing an estimate for the change in thermal conductivity in the void, we might expect a burn velocity that is at best in the range $0.1-1 \mathrm{~m} / \mathrm{s}$. Thus in this limit we are left with a burn velocity that is one or possibly even two orders of magnitude too low to support pumped convective burn.

\subsubsection{Single-step Arrhenius with Constant Heat Flux}

There are analytic models in the literature, all the way back to the "classical" Frank-Kamenetskii and Semenov models, which incorporate single-step Arrhenius kinetics with various simplifying assumptions [7, Ch. 4]. However, we have not (at least so far) found the exact calculation given below in the literature. Even if it turns out not to be new, it is worthwhile to show it in some detail so that the assumptions used are (hopefully) clearly stated.

We again start by considering the HE in a limit where convection is not important. This sounds like a strange assumption when considering something called convective burn, but recall that in our pumped convective burn model, the dominant convection is of air and fully reacted products through the voids. Transverse convection from a burning grain into the void space is handled via a boundary interaction; fluid-phase $\mathrm{HE}$ is assumed to convect quickly enough into the void space that it is treated as a volume source in the void, rather than following the convection explicitly. There is an implied boundary between fluid-phase and solid-phase material and the burn model applies (thus far) only to the solid phase.

Thus, as presented in Section 5.3, we treat the HE with transverse thermal conduction and a heat source from reacting HE, specified by a first-order Arrhenius term, proportional to an unreacted fraction $f$. The evolution of $f$ itself is governed by the same rate term. (In this section we are referring only to HE and will therefore drop all the "HE" subscripts.) 


$$
\begin{aligned}
& \rho C \frac{\partial T}{\partial t}=\kappa \frac{\partial^{2} T}{\partial x^{2}}+U_{o} f \nu e^{-T_{a} / T} \\
& \frac{\partial f}{\partial t}=-f \nu e^{-T_{a} / T}
\end{aligned}
$$

where $v$ is the rate constant, $T_{a}$ is the activation temperature, and $U_{o}$ is the net energy release per unit volume of HE.

If we change to a new $\mathrm{x}$-coordinate moving with a fixed speed $\mathrm{v}$ and look for a steady-state burn solution in this frame, we get:

$$
\begin{aligned}
& -\rho C v \frac{d T}{d x}=\frac{d^{2} T}{d x^{2}}+U_{o} f \nu e^{-T_{a} / T} \\
& -v \frac{d f}{d t}=-f \nu e^{-T_{a} / T}
\end{aligned}
$$

Exploiting the presence of the same combination of factors in right-hand sides of both equations, we can write the conduction equation in the form:

$$
-\rho C v \frac{d T}{d x}=\kappa \frac{d^{2} T}{d x^{2}}+U_{o} v \frac{d f}{d x}
$$

which can be integrated from some point $x$ to positive infinity (representing an undisturbed physical location far ahead of the burn front) where $\mathrm{f}=1, \mathrm{~T}=\mathrm{T}_{\mathrm{i}}$, and $\mathrm{dT} / \mathrm{dx}$ approaches 0 :

$$
\frac{\kappa}{U_{o} v} \frac{d T}{d x}=1-f-\frac{\rho C}{U_{o}}\left(T-T_{i}\right)
$$

The system is autonomous and $\mathrm{f}$ has a very well defined range from 0 to 1 , so we eliminate $\mathrm{x}$ and switch to $f$ as the independent variable, leaving a single ODE:

$$
\frac{\kappa}{U_{o} v^{2}} \frac{d T}{d f}=\frac{1-f-\frac{\rho C}{U_{o}}\left(T-T_{i}\right)}{f \nu e^{-T_{a} / T}}
$$

In the case where the domain of interest for $x$ extended to negative infinity, we would expect $\mathrm{dT} / \mathrm{dx}$ to approach zero along with $\mathrm{f}$; by inspecting the equation labeled (a), we can see that (T$\left.\mathrm{T}_{\mathrm{i}}\right) \rho \mathrm{C} / \mathrm{U}_{\mathrm{o}}$ must go to 1 in the same limit. This introduces a useful scale temperature that we use to define several normalized quantities:

$$
\begin{array}{rlrl}
\hat{T} & \equiv\left(T-T_{i}\right) \rho C / U_{o} & \hat{T}_{a} & \equiv T_{a} \rho C / U_{o} \\
\hat{T}_{i} \equiv T_{i} \rho C / U_{o} & V^{2} \equiv \frac{\rho C v^{2}}{\kappa \nu} e^{\hat{T}_{a} /\left(1+\hat{T}_{i}\right)}
\end{array}
$$

Over a broad range of applications, the normalized activation temperature is greater than 1 and the normalized initial temperature is $<<1$. Introducing these into the ODE yields the final form: 


$$
\frac{d \hat{T}}{d f}=V^{2} \frac{1-f-\hat{T}}{f} e^{\hat{T}_{a}\left(\frac{1}{\hat{T}+\hat{T}_{i}}-\frac{1}{1+\hat{T}_{i}}\right)}
$$

Let us start by considering the full-domain case where $\mathrm{x}$ ranges from negative to positive infinity and $\mathrm{f}$ ranges from 0 to 1 . Physically this is "perfect confinement," with no transverse convection and no heat flux entering or leaving the system behind the burn wave. In this case, the first-order ODE must satisfy two constraints, namely that the normalized temperature asymptote to 0 far ahead of the wave and asymptote to 1 behind it. The only way to satisfy both constraints with a first-order system is to allow $\mathrm{V}$ to be an eigenvalue in the problem, which is what will determine the burn velocity.

Hunt and peck with a simple spreadsheet calculation (where it is numerically more friendly to work in terms of $\mathrm{df} / \mathrm{dT}$ than $\mathrm{dT} / \mathrm{df}$ ) enables a fairly quick estimate for the eigenvalue $\mathrm{V}$ for various values of normalized $\mathrm{T}_{\mathrm{a}}$. Figure 5.4.1 shows some sample curves of normalized temperature versus f, and Figure 5.4.2 shows the resulting estimated eigenvalues. For all cases, the normalized initial temperature is fixed at 0.05 .

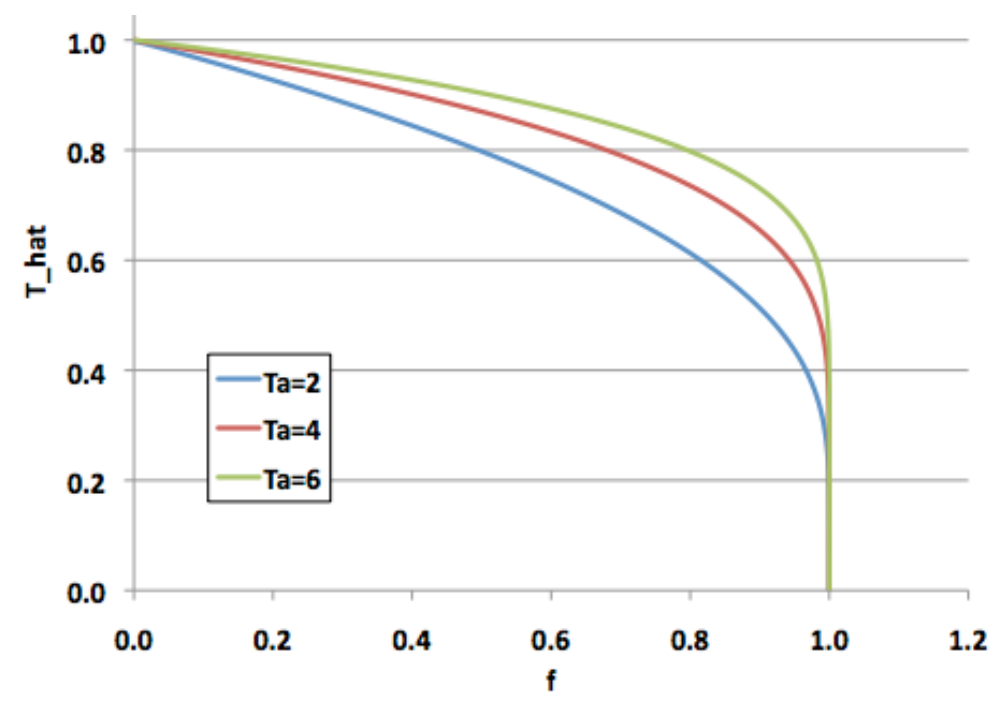

Figure 5.4.1. Plots of normalized temperature versus $f$ for three values of normalized activation temperature, in the "perfect confinement" limit. 


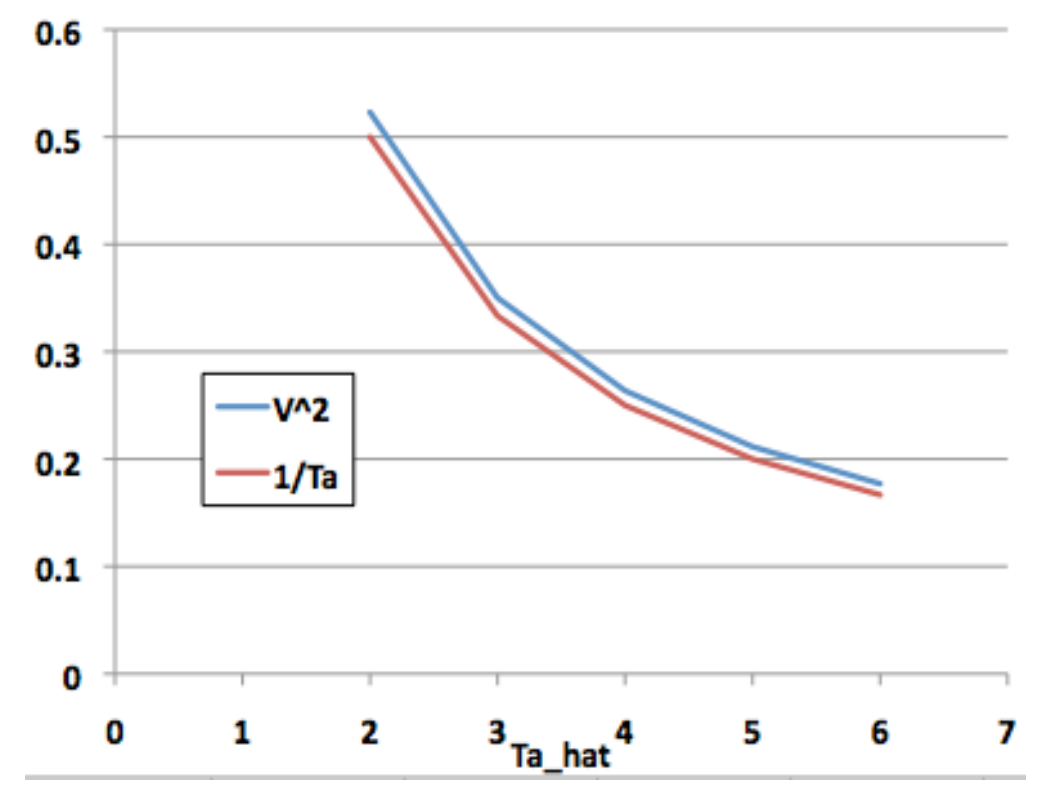

Figure 5.4.2. Estimated eigenvalues, as a function of normalized activation temperature. Also shown is a comparison to the fit $V^{2} \sim 1 / T_{a}$.

Using the fit $\mathrm{V}^{2} \sim 1 / \mathrm{T}_{\mathrm{a}}$ (normalized), the burn velocity can be calculated from the eigenvalue by:

$$
v=\sqrt{\frac{\kappa \nu e^{-\hat{T}_{a}}}{\rho C \hat{T}_{a}}}, \text { in } \mathrm{m} / \mathrm{s}
$$

A sample table of values is shown below, using the nominal HE parameters given previously.

\begin{tabular}{|c|c|}
\hline Normalized $\mathbf{T}_{\mathbf{a}}$ & $\mathbf{v}(\mathbf{m} / \mathbf{s})$ \\
\hline 2 & 924 \\
\hline 3 & 458 \\
\hline 4 & 240 \\
\hline 5 & 130 \\
\hline 6 & 72 \\
\hline
\end{tabular}

For the model parameters given earlier, the normalized $\mathrm{T}_{\mathrm{a}}$ is about 3 , but even at 5 we see that the burn rate is an order of magnitude higher than what we claim is sufficient for pumped convective burn. However, the "perfect confinement" case is not (of course) the whole story.

Let us now consider more realistic confinement. Inspection of the original model shows that the single-step, first-order Arrhenius model cannot completely consume the reactant in finite time. This means in the steady-wave case, the material behind the wave is not burned in a finite length of space. Thus we must choose a location (and corresponding non-zero value of $f$ ) that we define to be the "edge" of the burning HE. Upstream of that edge is fluid-phase material in the voids; downstream is (mostly) solid grain material. There is no clear choice of the correct value for $f$ at this location and we can only hope there is a range where the answer is otherwise relatively insensitive to it. We will denote this value by $f_{0}$. 
The only change to the formulation already given is that at the edge, there is heat flux into or out of the volume by means of convective heat transfer with the void. We replace the condition that the normalized $\mathrm{T}=1$ at $\mathrm{f}=0$ with:

$$
-\left.\kappa \frac{d T}{d x}\right|_{x=x\left(f_{o}\right)}=h\left(T_{e x t}-T\right)
$$

Introducing this condition into equation (a) above and defining a normalized external temperature:

$$
\hat{T}_{e x t} \equiv\left(T_{e x t}-T_{i}\right) \rho C / U_{o}
$$

produces the desired result:

$$
\left.\hat{T}\right|_{f_{o}}=\frac{(\rho C v / h)\left(1-f_{o}\right)+\hat{T}_{e x t}}{1+\rho C v / h}
$$

For reasonable ranges of parameters, $\rho \mathrm{Cv} / \mathrm{h}>>1$ which means the leading-order behavior of the boundary condition is:

$$
\left.\hat{T}\right|_{f_{o}} \approx 1-f_{o}
$$

which is curiously insensitive to the external temperature. If $\mathrm{f}_{\mathrm{o}}$ is chosen to be at a location where the HE is mostly burned, then we do achieve relative insensitivity to the precise value since "mostly burned" means $f_{o}<<1$. This returns us to the normalized $T=1$ condition, except it is imposed at a non-zero value of $\mathrm{f}$. Note, however, that the corrections to these approximate forms need to be included when calculating the initial slope, due to cancellation of the leadingorder terms.

Figure 5.4.3 shows how the peak temperature degrades as the boundary is moved closer to the burn front. Figure 5.4.4 shows how the burn velocity is affected. For all results shown, the ratio $\rho \mathrm{Cv} / \mathrm{h}$ is fixed at 1000 . (Note that the boundary condition is itself actually a function of the burn velocity; however, since the leading-order behavior of $\mathrm{v}$ does not vary tremendously, the uncertainty in the true value of $h$ enables us to pretend the ratio constant.) 


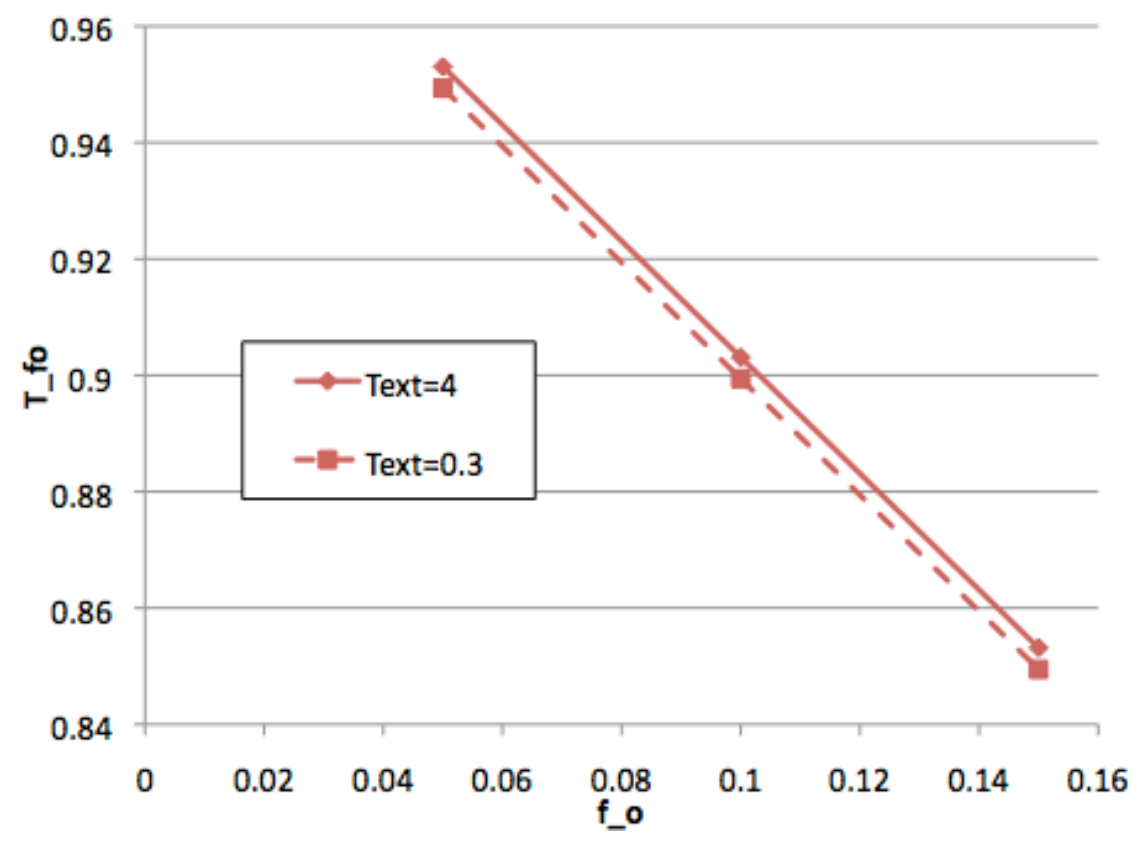

Figure 5.4.3. Change in peak normalized temperature as the finite heat flux boundary is moved into the reaction zone of the grain (increasing $f_{0}$ ).

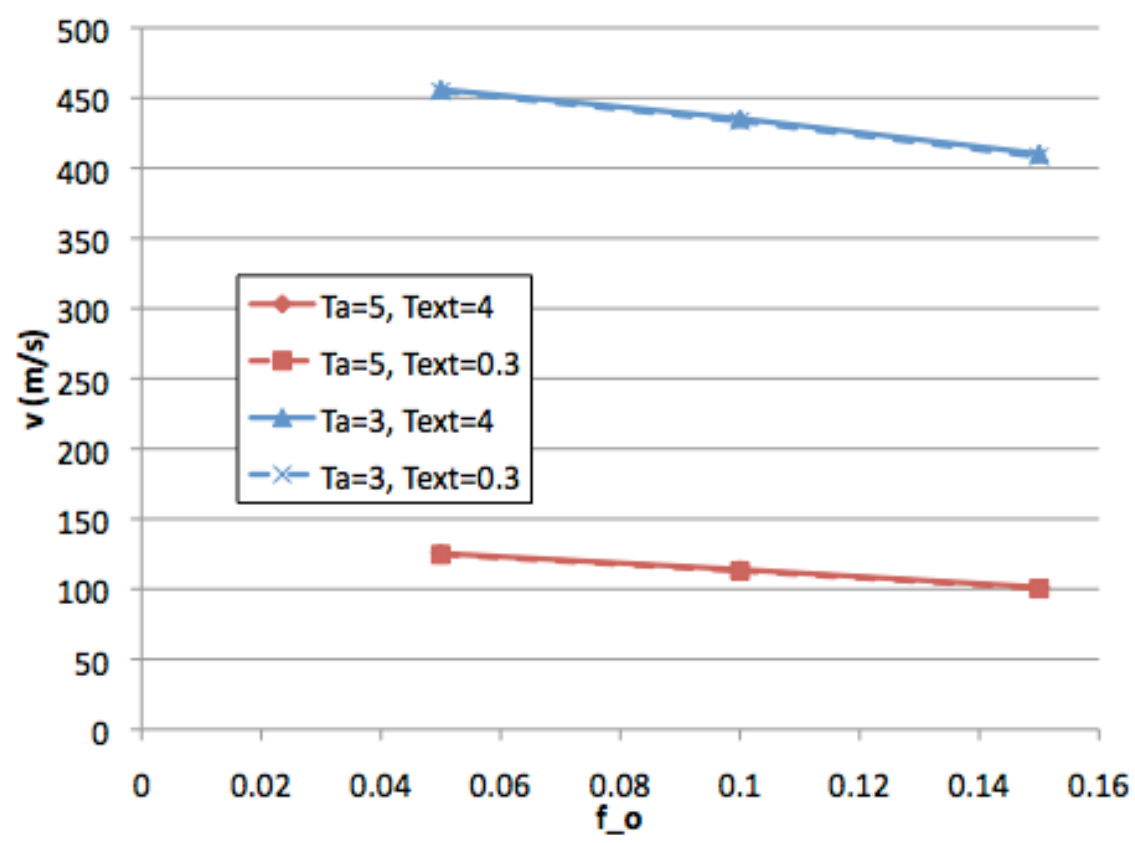

Figure 5.4.4. Dependence of the burn velocity on other parameters in the finite heat-flux case.

\subsubsection{Concluding Remarks on Burn Velocity}

We have presented two models that make very different assumptions about a burn wave propagating through an individual grain. In one limit, a very slow velocity of the order $0.1-1 \mathrm{~m} / \mathrm{s}$ results; in the other, numbers more on the order of $100-500 \mathrm{~m} / \mathrm{s}$ result. It seems reasonable to choose a middle-of-the-road value such as $10 \mathrm{~m} / \mathrm{s}$ for further exploration, as will be done in the 
next section. However, a better model of grain consumption, that actually treats the transverse convection into the void space, is clearly the direction that must be taken in future work. Section 5.6 takes up the question of endothermic effects by way of multi-step models.

\subsection{Some Cylindrical Time-Dependent Threshold Results}

Given a convective burn model that operates in terms of a constant burn velocity in the grains, and having spent considerable effort justifying what is still a tentative estimate for what a typical velocity might be, we finally present some results of the complete model from Section 5.3. The $\mathrm{C}++$ code used to produce these results is listed in Appendix A.

The following results use the hybrid (constant Prandtl number) Dittus-Boelter heat transfer model discussed earlier, and a hard-threshold HE model where no reaction takes place until the edge temperature exceeds $1000 \mathrm{~K}$, at which point mass and internal energy are released into the void at a constant rate until the grain is consumed in a time $r_{\mathrm{HE}} / \mathrm{v}_{\text {burn. }}$. Many combinations of parameters could be investigated; only a few are presented here.

First, we do a simple threshold study of input energy. We compare an input of 75 and $88 \mathrm{~mJ}$ (over a 100-mil arc length) in the following series of figures. Figure 5.5.1 compares the temperature in the void space (labeled "pore" in the figures). The scale of the figures is set by the peak heating of the source, which is tens of $\mathrm{eV}$; it therefore looks counterintuitive that the temperature drops quickly in time in the higher-energy $88 \mathrm{~mJ}$ case. However, this is because the reacted $\mathrm{HE}$ is cooler than the low-density air plasma it displaces as the HE burns; it is nevertheless still several thousand degrees. In the $75 \mathrm{~mJ}$ case, the cooler products are never introduced into the void space and the temperature stays higher longer. Note that this is the first reasonably first-principles model to predict ESD threshold anywhere in the ballpark observed experimentally; past attempts have generally resulted in extremely tiny values.
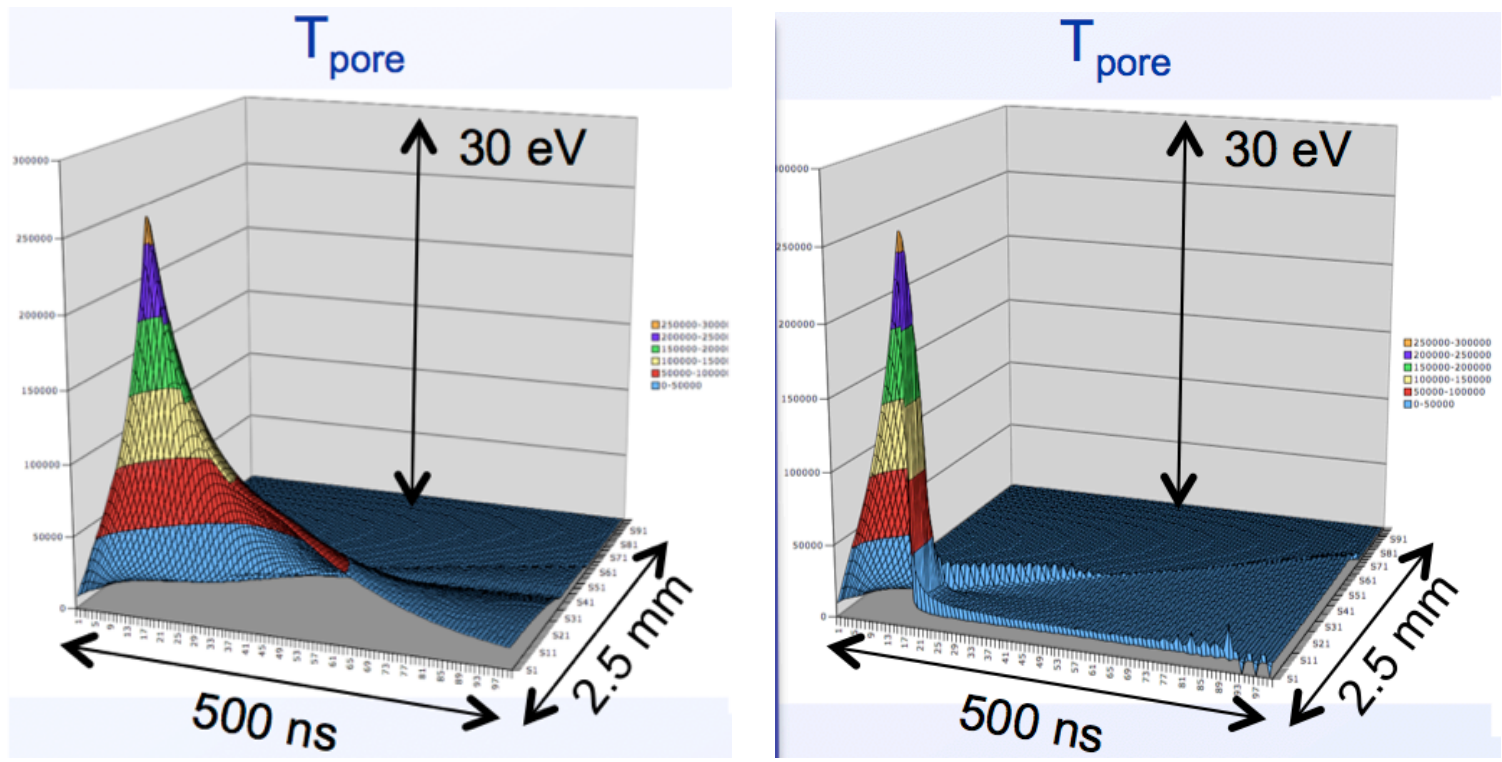

Figure 5.5.1. Temperature of the fluid in the void space below (left, $75 \mathrm{~mJ}$ input) and above (right, $88 \mathrm{~mJ}$ input) a calculated threshold. 
Figure 5.5.2 compares the edge temperature of the HE grains, and here it is clear why a distinct response threshold exists: it is a direct reflection of the hard ignition threshold in the grains. The $75 \mathrm{~mJ}$ case never exceeds $1000 \mathrm{~K}$ at the grain surface. Obviously it is important future work to see what behavior arises with a "softer" initiation model.
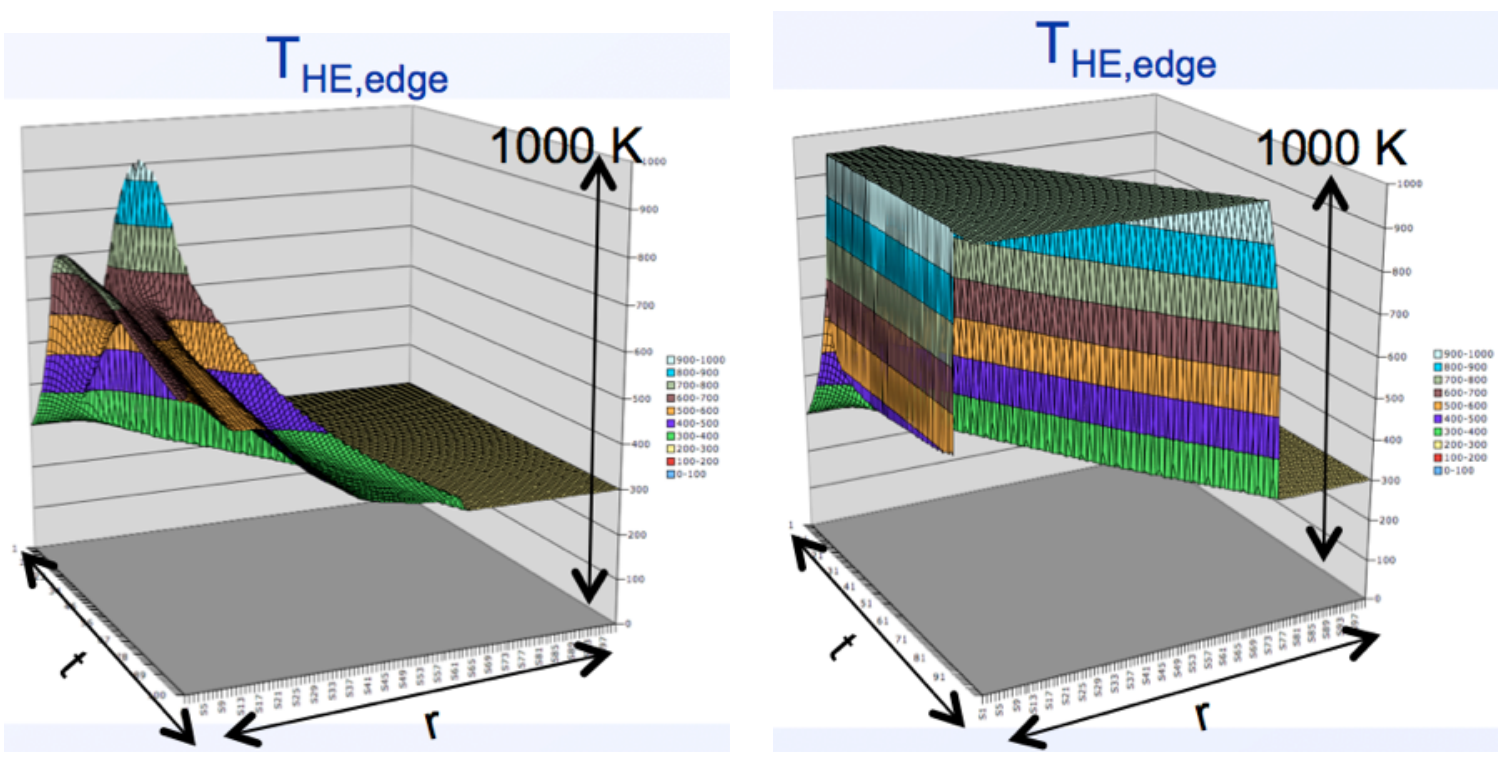

Figure 5.5.2. The edge temperature of the HE grains. The below-threshold case (left, $75 \mathrm{~mJ}$ input) never exceeds $1000 \mathrm{~K}$, whereas the above threshold case (right, $88 \mathrm{~mJ}$ ) does.

Figure 5.5.3 shows the most visibly obvious difference in the output data. Once the HE ignites given the higher input energy, reaction products are injected into the void space, driving the average density much higher than the initial value. In the ideal gas model used, the density gradient in turn produces a pressure gradient that drives the convection through the pores.
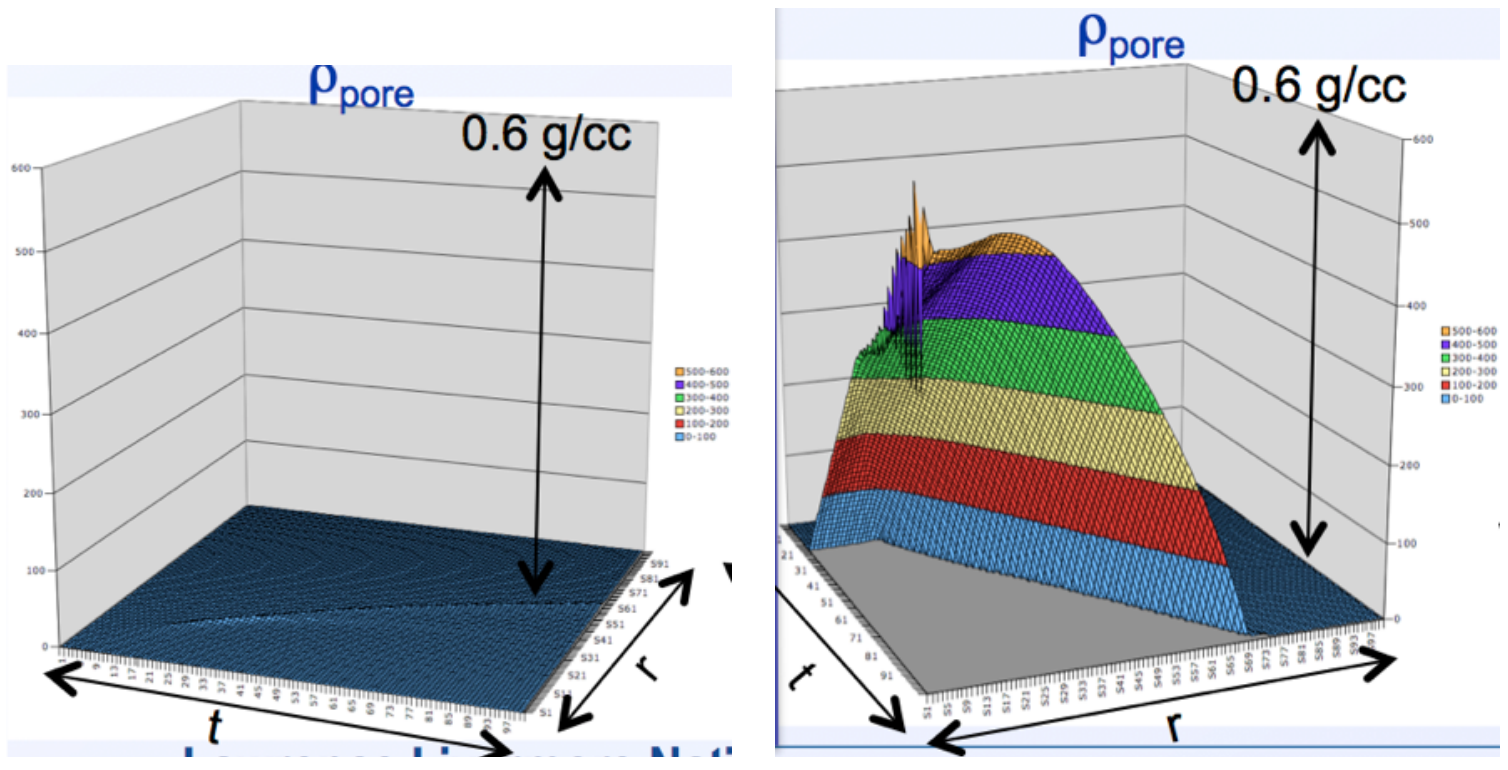

Figure 5.5.3. The fluid density in the void space, showing the clear difference in behavior between the below-threshold case (left, $75 \mathrm{~mJ}$ input) where there is only air plasma from the 


\section{initial discharge, and the above-threshold case (right, $88 \mathrm{~mJ}$ input) where significant mass is injected into the voids.}

Figure 5.5.4 compares the flow velocity through the void space and is the most interesting from a phenomenological point of view. In the below-threshold case, the flow velocity starts high but decays once the input source is turned off. In the above-threshold case, the velocity stays high and roughly constant, and is clearly sufficient to traverse a detonator-sized volume in 2-3 microseconds.
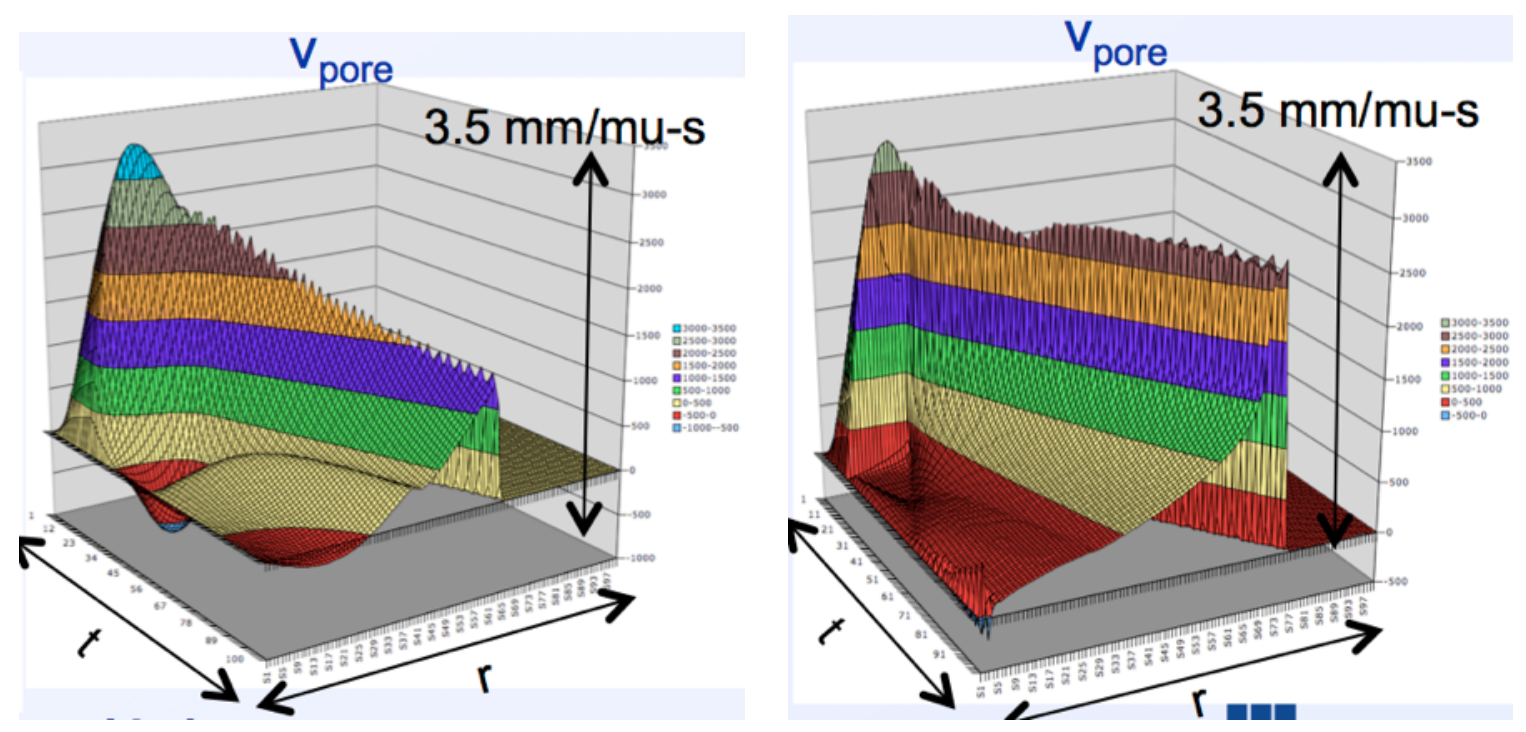

Figure 5.5.4. Fluid velocity in the void space, showing the decay following the initial $75 \mathrm{~mJ}$ input (left) compared to the sustained propagation in the above-threshold $88 \mathrm{~mJ}$ input case (right).

Figure 5.5.5 shows the thresholds obtained from the model at different pulse lengths (at fixed input energy, a shorter pulse length corresponds to a higher input power). It is interesting to see that the model does indeed show a power-dependent threshold in the regime of interest, which was indicated by early experiments and then not observed in later ones. This remains an open question.

Finally, Figure 5.5.6 shows a variation as the void and grain size are simultaneously varied. This is not exactly analogous to varying the specific surface area, since the latter appears only in the heat transfer term but the void and grain sizes appear in other terms. Nevertheless, the threshold does decrease as the grain decreases, which would imply an increase in surface area; this increase has been observed experimentally. 


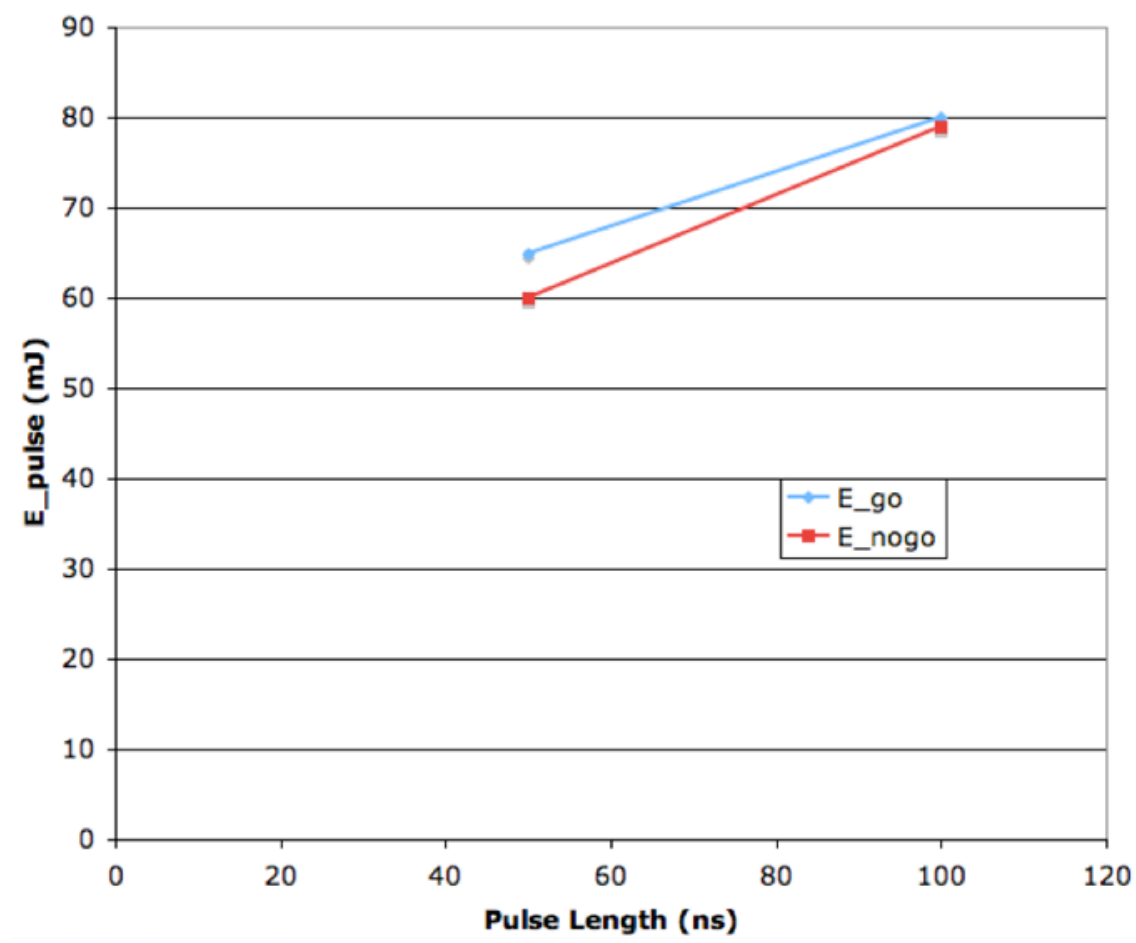

Figure 5.5.5. Effect of varying the pulse length, which varies the input power. A numerical threshold dependence is observed. The experimental results to date are not clear.

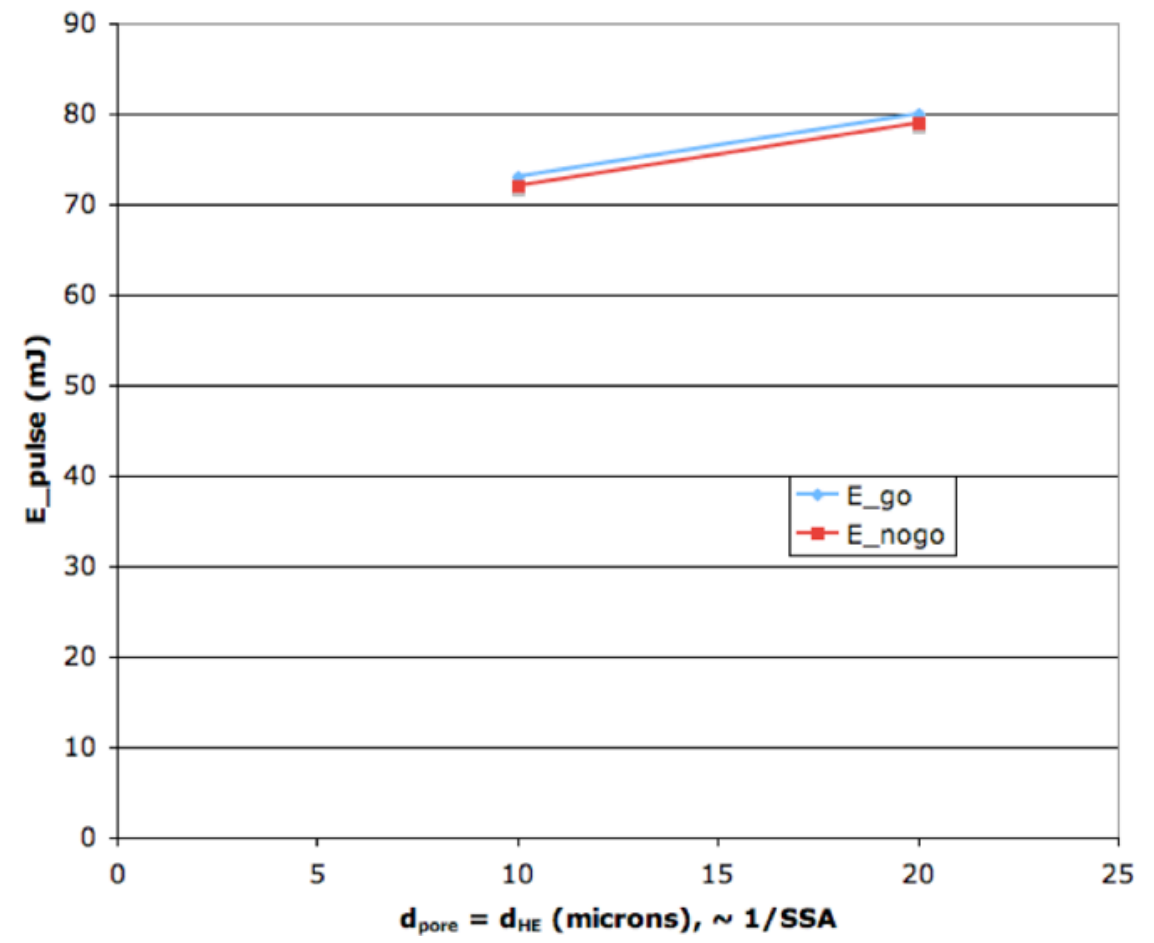

Figure 5.5.6. Effect of varying the grain and void size (which are kept equal to each other). 


\subsection{Burn Rates from Multi-Step Models}

The rate at which an individual HE grain burns is an important parameter with respect to being able to sustain a PCB wave (the quantitative role is explored in subsection 5.7). In the broader literature on convective burn of HE, good empirical correlations have been established for the burn rates [10]. However, these correlations are pressure-based and therefore lack connection to the underlying kinetics of the HE. It is also desirable to link the behavior of the burn rate to very specific HE properties such as endothermic barriers, and thus be able to make quantitative statements about the safety-relevant HE behavior.

To that end, in this subsection we explore calculations similar to those introduced in subsection 5.4.3, but for multi-step kinetics models that are intended to provide better-calibrated descriptions of real HEs. While the models themselves are still highly reduced relative to the full, complex reactions chains, much accumulated evidence shows that "binning" similar reactions into a smaller set of steps still does a very good job at reproducing observed behavior.

We will also compare the burn rates that result from such calculations with existing data.

\subsubsection{Eigenvalue Calculations with Existing Multi-Step Models}

The first multi-step model we consider is the Tran/Tarver/Whipple four-step model for PETN [8]. Using the same constant-property assumptions as in 5.4.3 and starting with the equations already moved to the frame of a steady burn wave moving at speed $\mathrm{v}_{\mathrm{b}}$, the desired system is:

$$
\begin{gathered}
-v_{b} \frac{d f_{1}}{d x}=-f_{1} \nu_{1} e^{-T_{1} / T}-\frac{1}{2} f_{1} f_{2} \nu_{2} e^{-T_{2} / T} \\
-v_{b} \frac{d f_{2}}{d x}=+f_{1} \nu_{1} e^{-T_{1} / T}-\frac{1}{2} f_{1} f_{2} \nu_{2} e^{-T_{2} / T}-f_{2}^{2} \nu_{3} e^{-T_{3} / T} \\
-v_{b} \frac{d f_{3}}{d x}=+f_{1} f_{2} \nu_{2} e^{-T_{2} / T}+f_{2}^{2} \nu_{3} e^{-T_{3} / T}-f_{3}^{2} \nu_{4} e^{-T_{4} / T} \\
-v_{b} \rho_{o} C \frac{d T}{d x}=\kappa \frac{d^{2} T}{d x^{2}}+Q_{1} f_{1} \nu_{1} e^{-T_{1} / T}+Q_{2} f_{1} f_{2} \nu_{2} e^{-T_{2} / T}+Q_{3} f_{2}^{2} \nu_{3} e^{-T_{3} / T}+Q_{4} f_{3}^{2} \nu_{4} e^{-T_{4} / T}
\end{gathered}
$$

Table 5.6.1 shows the model parameters; a positive sign on the reaction energy Q corresponds to an exothermic energy release. The first reaction is from pristine PETN (denoted with mass fraction $\mathrm{f}_{1}$ ) to solid-phase intermediates $\left(\mathrm{f}_{2}\right)$. The second has PETN reacting with the solid-phase intermediates to form gaseous intermediates $\left(f_{3}\right)$. The third is direct decomposition of the solidphase intermediates to gaseous intermediates. The last step takes the gaseous intermediates to the final gaseous end products $\left(f_{4}=1-f_{1}-f_{2}-f_{3}\right)$. 


\begin{tabular}{|c|c|c|c|}
\hline $\mathbf{i}$ & $\mathbf{T}_{\mathbf{i}}(\mathbf{K})$ & $\mathbf{v}_{\mathbf{i}}\left(\mathbf{s}^{\mathbf{- 1}}\right)$ & $\left.\mathbf{Q}_{\mathbf{i}} / \mathbf{\rho}_{\mathbf{o}} \mathbf{( M J} / \mathbf{k g}\right)$ \\
\hline $\mathbf{1}$ & 23645 & $6.3 \mathrm{e} 19$ & -0.209 \\
\hline $\mathbf{2}$ & 21444 & $1.65 \mathrm{e} 18$ & 0.523 \\
\hline $\mathbf{3}$ & 18000 & $4.3 \mathrm{e} 15$ & 0.627 \\
\hline $\mathbf{4}$ & 15250 & $7.9 \mathrm{e} 13$ & 5.44 \\
\hline
\end{tabular}

Table 5.6.1. Model parameters for four-step Arrhenius kinetics for PETN [8]. The specific heat is $1100 \mathrm{~J} / \mathrm{kg}$ $\mathrm{K}$, density is $1760 \mathrm{~kg} / \mathrm{m}^{3}$, and thermal conductivity is $0.25 \mathrm{~W} / \mathrm{m}-\mathrm{K}$.

As $x$ goes to positive infinity, we must have $\mathrm{f}_{1}=1, \mathrm{f}_{2}=0, \mathrm{f}_{3}=0$, and $\mathrm{T}=\mathrm{T}_{\mathrm{i}}=300 \mathrm{~K}$. If we introduce a fictitious mass fraction $\mathrm{f}_{0}$ which satisfies:

$$
-v_{b} \frac{d f_{0}}{d x}=-f_{1} \nu_{1} e^{-T_{1} / T}
$$

and $\mathrm{f}_{0}=\mathrm{f}_{1}$ at positive infinity, it then happens that the individual reaction terms on the right-hand sides of the reaction rate equations form an invertible system from which we can express the reaction rate terms in the temperature equation entirely in terms of combinations of the various $\mathrm{df}_{\mathrm{i}} / \mathrm{dx}, \mathrm{i}=0 \ldots 3$. Combining this property with the boundary condition at positive infinity allows us to integrate the temperature equation once:

$$
\begin{array}{r}
\frac{\kappa}{v_{b} \rho C} \frac{d T}{d x}=T_{i}-T+\frac{Q_{1}+Q_{3}+Q_{4}}{\rho C}-\frac{1}{\rho C}\left(f_{0}\left(Q_{1}-2 Q_{2}+2 Q_{3}\right)+\right. \\
\left.f_{1}\left(2 Q_{2}-Q_{3}+Q_{4}\right)+f_{2}\left(Q_{3}+Q_{4}\right)+f_{3} Q_{4}\right)
\end{array}
$$

This leaves an autonomous first-order system that becomes amenable to solution with spreadsheet-level tools by transforming the independent variable from $\mathrm{x}$ to $\mathrm{T}$ :

$$
\begin{aligned}
& \frac{d f_{0}}{d T}=f_{1} \nu_{1} e^{-T_{1} / T} /\left(v_{b} d T / d x\right) \\
& \frac{d f_{1}}{d T}=\left(f_{1} \nu_{1} e^{-T_{1} / T}+\frac{1}{2} f_{1} f_{2} \nu_{2} e^{-T_{2} / T}\right) /\left(v_{b} d T / d x\right) \\
& \frac{d f_{2}}{d T}=\frac{f_{2}^{2} \nu_{3} e^{-T_{3} / T}-f_{1} \nu_{1} e^{-T_{1} / T}+\frac{1}{2} f_{1} f_{2} \nu_{2} e^{-T_{2} / T}}{v_{b} d T / d x} \\
& \frac{d f_{3}}{d T}=\frac{f_{3}^{2} \nu_{4} e^{-T_{4} / T}-f_{2}^{2} \nu_{3} e^{-T_{3} / T}-f_{1} f_{2} \nu_{2} e^{-T_{2} / T}}{v_{b} d T / d x}
\end{aligned}
$$

In practice, to avoid having $\mathrm{dT} / \mathrm{dx}$ identically zero at positive infinity, we cheat ever so slightly by setting $f_{1}=1-\varepsilon$ there, where the final answer turns out not to be sensitive to the value of $\varepsilon$. We used $10^{-6}$. The question is, what is the range of T? And under what circumstances does it remain monotonic? The answer to these questions is tied to the "cold boundary problem," defined by Penner [11] and studied at least as far back as von Karman [see the references within 11]. In short, there is no physical realization of the assumption that the steady wave is actually 
established all the way back to negative infinity. The physical solution must be tied to the hydrodynamics of boundary conditions that remain coupled to the burning of the grain over all time scales relevant to the burning; this is why pressure-based correlations work, because the pressure of the larger-scale flow field remains coupled to the problem.

For this level of calculation, however, we solve the cold boundary problem by assuming the upstream material remains motionless. This could only be true if the burn rate was fast compared to the inertial time scales of the motion (and it most certainly is not), or if confinement of the reaction products is extremely strong. We will compare the results to a case where the requisite high pressures can be maintained, namely in a diamond anvil cell (DAC).

With perfect confinement, the temperature will increase monotonically until all steps of the reaction are complete and a limit of zero heat flux is reached. From the integrated form of the temperature equation:

$$
T_{-\infty}=T_{i}+\frac{Q_{1}+Q_{3}+Q_{4}+2 Q_{2} f_{0}}{\rho C}
$$

Using an a priori assumption that $\mathrm{f}_{0}$ becomes small, this ideal zero heat flux limit is reached at about $\mathrm{T}=5650 \mathrm{~K}$. (At the end of the calculations, $\mathrm{f}_{0}$ asymptotes to about 0.025 , justifying the initial assumption.)

With the range of $\mathrm{T}$ established, the burn rate becomes an eigenvalue which at which the reacted fractions reach their steady value of $\mathrm{f}_{1}=0, \mathrm{f}_{2}=0, \mathrm{f}_{3}=0, \mathrm{f}_{4}=1$ just as $\mathrm{dT} / \mathrm{dx}$ is reaching 0 . Figure 5.6.1 shows the resulting profiles of reacted fraction as a function of temperature over the desired range, for PETN. The resulting burn rate is $334 \mathrm{~m} / \mathrm{s}$. Under the perfect-confinement assumption for material starting at atmospheric pressure, the fully reacted material ends up at pressure of roughly 2-3 GPa if one were to assume an ideal equation of state and an average molecular weight of products between 18 and 44 (water to $\mathrm{CO}_{2}$ ). Note that even under these idealized confinement conditions, the pressure is well below the $\sim 30 \mathrm{GPa}$ Chapman-Jouguet pressure of detonating PETN.

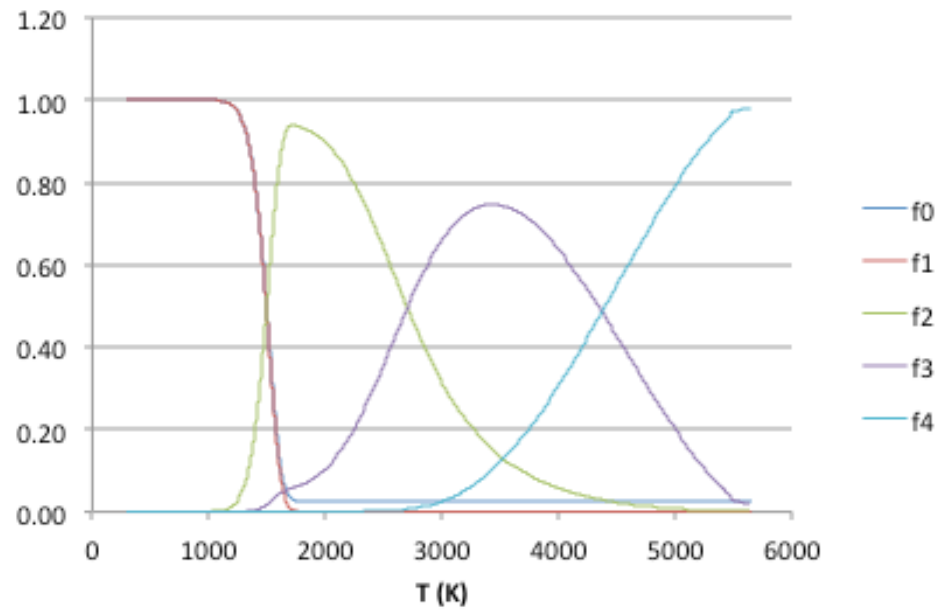

Figure 5.6.1. Steady-burn-wave profiles of reacting mass fractions versus temperature, based on a four-step Arrhenius model for PETN, assuming idealized confinement. The burn velocity eigenvalue is $334 \mathrm{~m} / \mathrm{s}$. 
We also consider the same calculation done on the three-step model of Tarver/Chidester/Nichols for TATB [14]. This model is a serial evolution from TATB to a first solid intermediate (first order), from the first to a second solid intermediate (first order), and then from the second intermediate to gaseous end products (second order). Because of this serial structure, no additional quantities equivalent to $f_{0}$ for PETN need to be introduced prior to inverting the rates and integrating the temperature equation. The first two steps are endothermic and the temperature of the end state is much lower, $\mathrm{T}=2580 \mathrm{~K}$. Table 5.6.2 shows the model parameters and Figure 5.6.2 shows the results of the eigenvalue calculation for the burn rate, yielding a value of $1.7 \mathrm{~m} / \mathrm{s}$.

\begin{tabular}{|c|c|c|c|}
\hline $\mathbf{i}$ & $\mathbf{T}_{\mathbf{i}} \mathbf{( K )}$ & $\boldsymbol{v}_{\mathbf{i}}\left(\mathbf{s}^{\mathbf{- 1}}\right)$ & $\left.\mathbf{Q}_{\mathbf{i}} / \boldsymbol{\rho}_{\mathbf{o}} \mathbf{( M J} / \mathbf{k g}\right)$ \\
\hline $\mathbf{1}$ & 30188 & $7 . \mathrm{e} 20$ & -0.209 \\
\hline $\mathbf{2}$ & 21131 & $8.75 \mathrm{e} 12$ & -0.209 \\
\hline $\mathbf{3}$ & 17000 & $4.36 \mathrm{e} 11$ & 2.93 \\
\hline
\end{tabular}

Table 5.6.2. Model parameters for three-step Arrhenius kinetics for TATB [14]. The specific heat is 1100 $\mathrm{J} / \mathrm{kg}-\mathrm{K}$, density is $1800 \mathrm{~kg} / \mathrm{m}^{3}$, and thermal conductivity is $0.25 \mathrm{~W} / \mathrm{m}-\mathrm{K}$.

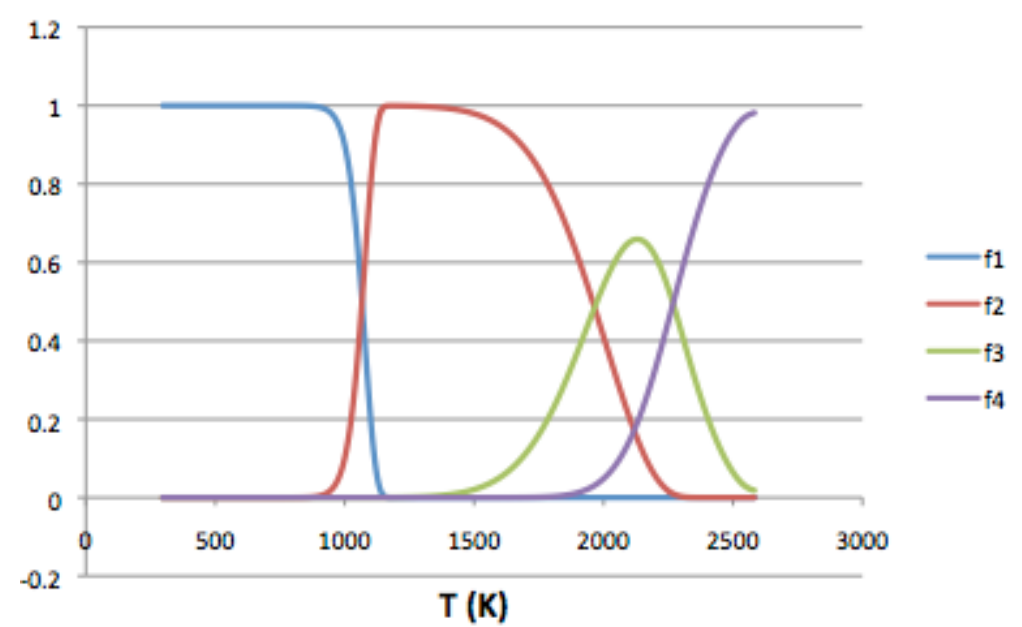

Figure 5.6.2. Steady-burn-wave profiles of reacting mass fractions versus temperature, based on a three-step Arrhenius model for TATB, assuming idealized confinement. The burn velocity eigenvalue is $1.7 \mathrm{~m} / \mathrm{s}$.

As simple as these models are, it is interesting to note how the values bracket the numerically estimated threshold of roughly $10 \mathrm{~m} / \mathrm{s}$ for sustaining the PCB phenomenon. TATB, of course, has well-documented insensitivity to anything other than mechanical shock - including to electrical insults.

\subsubsection{Comparison with DAC Data}

One experimental regime where confinement pressures of order of our highly idealized picture can actually occur is with a diamond anvil cell (DAC). Let us assume that as we increase pressure at fixed temperature, the corresponding increase in material density will scale the reaction rates for anything other than simple first-order decomposition, since the corresponding collision rates will increase. Consideration of how the mass fractions emerge from rate equations 
based on number density shows that the rates should scale with the square of ratio of compressed to nominal density. When we compare to the DAC results, we assume the density ratio is equal to the ratio of the test pressure to the baseline pressures reached in the results of the previous section.

The DAC data in question is for PETN [12] and TATB [13]. Figure 5.6.3 below shows the comparison of predicted and measured burn rates for PETN, and Figure 5.6.4 for TATB. The ellipses span the range of unknown molecular weight of the final products, from 18 to 44 . Each HE type compares well in one aspect and not in another. In the case of PETN, the predicted rates are offset from the measured values by roughly $250 \mathrm{~m} / \mathrm{s}$. However, the linear slope of the trend with increasing pressure appears to match very well. For TATB, we see the opposite result: the burn velocity is certainly within the ballpark, but the trend with pressure is incorrect; the model appears to predict a roll-over that does not actually occur. However, given all of the other assumptions in the model (constant properties, no thermal conduction losses to sidewalls, etc.), the half-matches are intriguing.

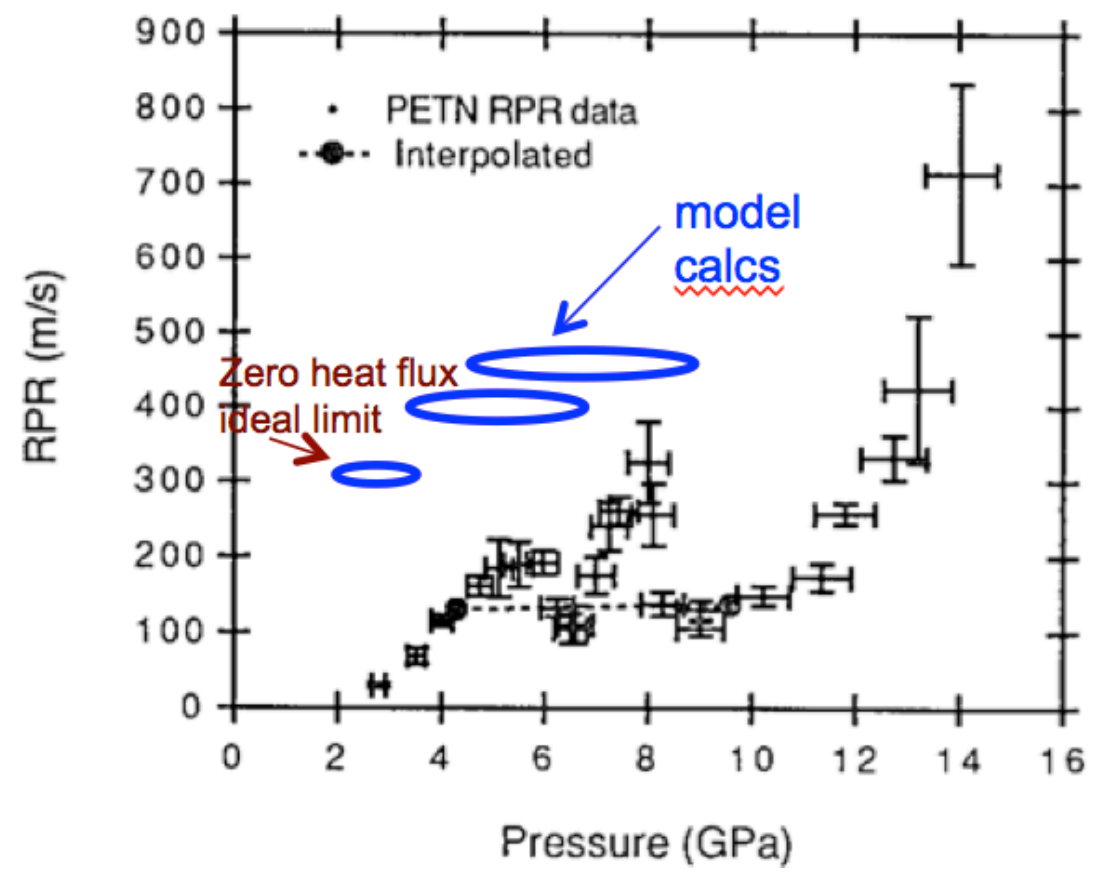

Figure 5.6.3. Measured reaction propagation rates (RPR) versus DAC pressure, for PETN. Data from Foltz, et al (1993) is in black, the modeled results are the blue ellipses where the elongation spans the unknown average molecular weight of reaction products (the ends span 18 to 44 ). 


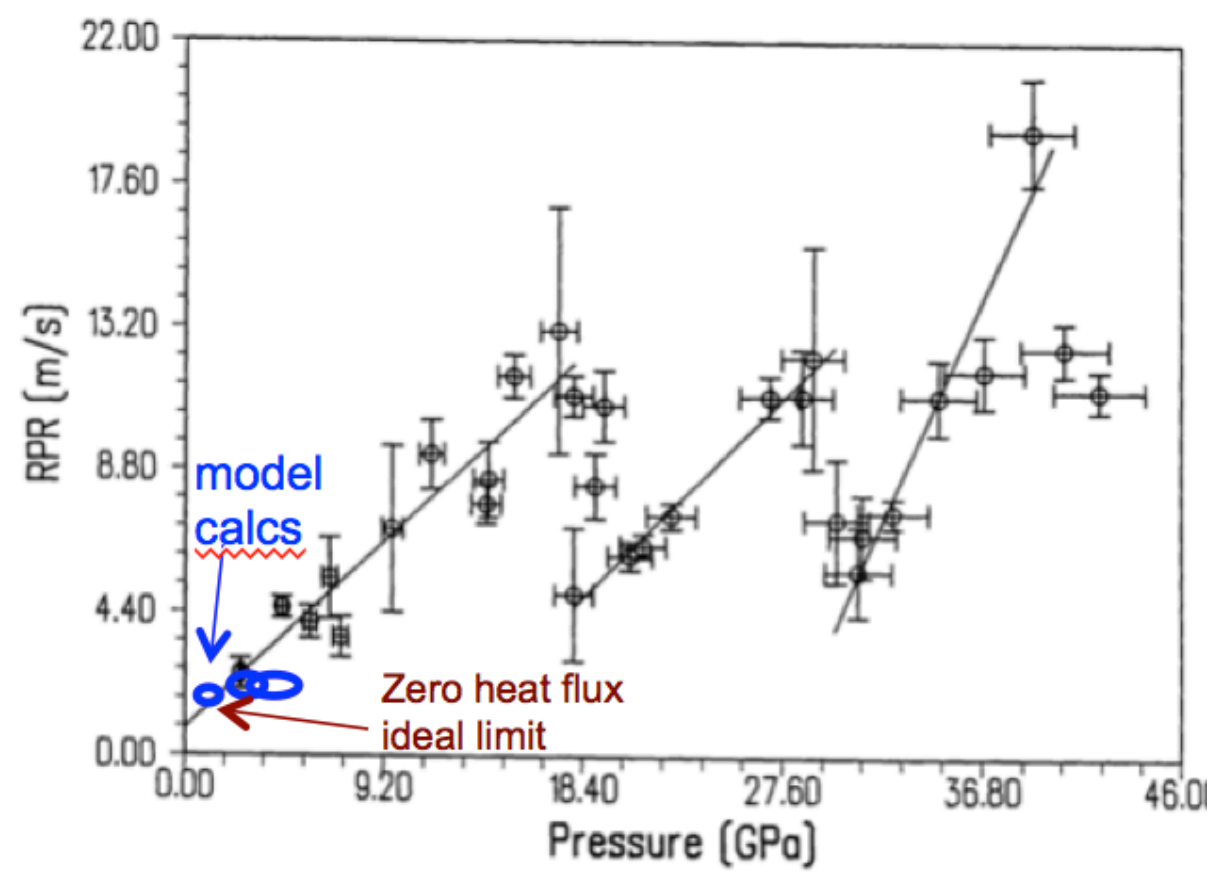

Figure 5.6.4. Measured reaction propagation rates (RPR) versus DAC pressure, for TATB. Data from Foltz, et al (1991) is in black, the modeled results are the blue ellipses where the elongation spans the unknown average molecular weight of reaction products (the ends span 18 to 44 ).

\subsection{Derivation of a Steady PCB Wave}

The simulations shown in subsection 5.5 are time-dependent, following the evolution of the PCB model starting with an input pulse into a quiescent system. Something resembling a steady wave appears to have formed by the end of the simulation. It is certainly possible that the numerical robustness and accuracy of the simulation are not yet up to par and that these results are artifacts of some sort; the simulation uses artificially large viscosity and thermal conduction coefficients and may well drive the results into a regime determined more by diffusive transport across a shock. It would be desirable to have some other means of studying and confirming such a wave.

In the limit of a hard-threshold HE model with a constant grain burn rate once reaction has commenced - the same limit used in the simulations - it is in fact possible to analytically derive the existence of a steady plane wave (the previous simulation was cylindrical) in the pumped convective burn regime. Separate numerical simulations employing a planar geometry, no thermal conduction, and an artificial viscosity that does not dominate in any region of interest have also been performed and match the analytic results fairly well. This wave has some unique properties:

- The underlying burning of the HE does not assume or require shock compression of solidphase material. The burn wave in the solid grains is a conductive burn wave.

- Compaction of low-density material is not required and not included in the model. (Again, whether compaction can or does actually occur over real-world timescales in a small, detonatorsized volume of HE is a separate question. It is not required to support this steady PCB wave.) 
- For typical parameters in something like half-dense PETN, the plane wave speed is slower than a detonation wave by a factor of 3-4 but much faster than any traditional convective burning scenario.

Understanding of this wave is not very deep at this time. Open questions for future pursuit pertain to the wave stability (especially in more realistic non-planar geometries) and the ability to couple to this wave in under- and over-driven scenarios. The latter question is especially intriguing in the context of the role of endothermic barriers. For example, quantitative proof that the properties of TATB result in a fundamental inability to DDT would be a valuable addition to our knowledge.

We break the remainder of this topic into three further subsections. Subsection 5.7.1 shows results of planar, time-dependent simulations. Subsection 5.7.2 is the mathematical derivation of the steady wave; it is certainly important from the perspective of maintaining a record of the method and assumptions, but can be safely skipped by those who find such mechanics uninteresting. Subsection 5.7.3 discusses some properties of the results.

\subsubsection{Planar Time-Dependent Simulations}

The planar model is not unlike that shown previously for the cylindrical case:

$$
\begin{gathered}
\frac{\partial \rho}{\partial t}+\frac{\partial}{\partial x} \rho v=\frac{2 \rho_{H} v_{b}}{d} H\left(T_{H}-T_{c r i t}\right) H\left(\frac{d_{H}}{2 v_{b}}-t_{\text {burn }}\right) \\
\frac{\partial}{\partial t} \rho v+\frac{\partial}{\partial x}\left(P+\rho v^{2}\right)=-\frac{f \rho v^{2}}{2 d} H\left(T_{c r i t}-T_{H}\right) \\
\frac{\partial}{\partial t}\left(\frac{P}{\gamma-1}+\rho v^{2} / 2\right)+\frac{\partial}{\partial x}\left(\frac{\gamma P}{\gamma-1}+\rho v^{2}\right)=\frac{2 U_{H} v_{b}}{d} H\left(T_{H}-T_{c r i t}\right) H\left(\frac{d_{H}}{2 v_{b}}-t_{b u r n}\right) \\
\left.\frac{\partial t_{b u r n}}{\partial t}=H\left(T_{H}-T\right) H\left(T_{c r i t}-T_{H}\right)+Q_{\text {ext }}(x) t\right) \\
\rho_{H} C_{H} \frac{\partial T_{H}}{\partial t}=\frac{h}{d_{e f f}}\left(T-T_{H}\right)
\end{gathered}
$$

A subscript of $\mathrm{H}$ refers to a solid-phase property of the HE. We relate the gas-phase pressure $\mathrm{P}$, internal energy per unit volume $\mathrm{U}$, and temperature $\mathrm{T}$ by:

$$
U=\frac{P}{\gamma-1 ;} \quad P=\frac{\rho k_{b} T}{m}
$$

There are several other simplifying assumptions: 
- Material properties, include the friction factor and heat transfer coefficient, are all constant.

- Heat transfer and frictional drag between the solid and gas phases must drop to zero by the time the solid phase has completely burned; here, we set these terms to zero as soon as burning begins.

- Burning is assumed to occur at a constant rate, as if the grains themselves had a planar form (transverse to the flow direction). Thus, a burn front of speed $\mathrm{v}_{\mathrm{b}}$ is propagating in from either "side" of a planar grain of size $d_{H}$, consuming it in a time $d_{H} / 2 v_{b}$.

- The characteristic void size $d$ and grain size $d_{H}$ do not vary in time, even when burning is taking place.

- The relevant heating of a grain prior to the onset of burning is assumed to take place uniformly in a thin layer of size $\mathrm{d}_{\mathrm{eff}}<\mathrm{d}_{\mathrm{H}}$.

- For ease of bookkeeping, we enforce continuity of air, solid HE, and reaction products by assuming the initial "air" and the reaction products have the same molecular weight $\mathrm{m}$, and furthermore that that weight is chosen such that $C_{H}=k_{b} /(\gamma-1) m$. This yields $m=19 m_{p}$ where $m_{p}$ is the mass of a proton, for $\mathrm{C}_{\mathrm{H}}=1100 \mathrm{~J} / \mathrm{K}-\mathrm{kg}$.

- As a result of the previous assumption and keeping the initial "air" density at the nominal value of $1.2 \mathrm{~kg} / \mathrm{m}^{3}$, the initial pressure is about $50 \%$ higher than standard conditions.

The quantity $\mathrm{U}_{\mathrm{H}}$ is the internal energy that would be released from a constant-volume burn of the $\mathrm{HE}$; in our idealized constant-property model, we estimate it by using the form:

$$
U_{H}=\rho_{H} C_{H} T_{\text {react }}
$$

where $T_{\text {react }}$ is some peak HE-dependent temperature. $T_{\text {crit }}$ is the hard-threshold temperature at which the HE begins to burn with a fixed burn front speed of $\mathrm{v}_{\mathrm{b}}$ into the grain.

For the results shown below, our baseline parameter set is:

Initial conditions (at $\mathrm{t}=0$ ):

$\rho=1.2 \mathrm{~kg} / \mathrm{m}^{3}$

$\mathrm{v}=0 \mathrm{~m} / \mathrm{s}$

$\mathrm{T}=300 \mathrm{~K}$

$\mathrm{T}_{\mathrm{H}}=300 \mathrm{~K}$

$\mathrm{t}_{\mathrm{burn}}=0$ (note this is a function of $\mathrm{x}$ )

Parameters:

$\gamma=1.4$

$\mathrm{m}=19 \times 1.67 \times 10^{-27} \mathrm{~kg}$

$\mathrm{f}=0.1$

$\mathrm{d}=1 . \mathrm{e}-5 \mathrm{~m}$

$\mathrm{d}_{\mathrm{H}}=1 . \mathrm{e}-5 \mathrm{~m}$

$\mathrm{d}_{\mathrm{eff}}=0.1 \mathrm{dH}=1 . \mathrm{e}-7 \mathrm{~m}$

$\kappa_{\text {air }}=0.024 \mathrm{~W} / \mathrm{m}-\mathrm{K}$

$\mathrm{h}=\mathrm{Nu} \kappa_{\text {air }} / \mathrm{d}, \mathrm{Nu}=16$

$\mathrm{v}_{\mathrm{b}}=100 \mathrm{~m} / \mathrm{s}$ 
$\rho_{\mathrm{H}}=1780 \mathrm{~kg} / \mathrm{m}^{3}$

$\mathrm{C}_{\mathrm{H}}=1100 \mathrm{~J} / \mathrm{K}-\mathrm{kg}$

$\mathrm{T}_{\text {react }}=5500 \mathrm{~K}$

$\mathrm{T}_{\text {crit }}=900 \mathrm{~K}$

The initial heating is provided by an external source of the form:

$$
Q_{\text {ext }}=\frac{E_{\text {tot }}}{t_{\text {pulse }} \pi r_{o}^{2} l} e^{-x^{2} / r_{o}^{2}} H\left(t_{\text {pulse }}-t\right)
$$

where we chose $E_{\text {tot }}=15 \mathrm{~mJ}, \mathrm{r}_{\mathrm{o}}=250$ microns, $1=2.5 \mathrm{~mm}$, and $\mathrm{t}_{\text {pulse }}=100 \mathrm{~ns}$.

We enforce a symmetry plane at $\mathrm{x}=0$ and keep conditions at the far end fixed, since it is timeisolated.

Consider the plots below of gas-phase density, velocity, temperature, and pressure, as well as the solid-phase temperature.

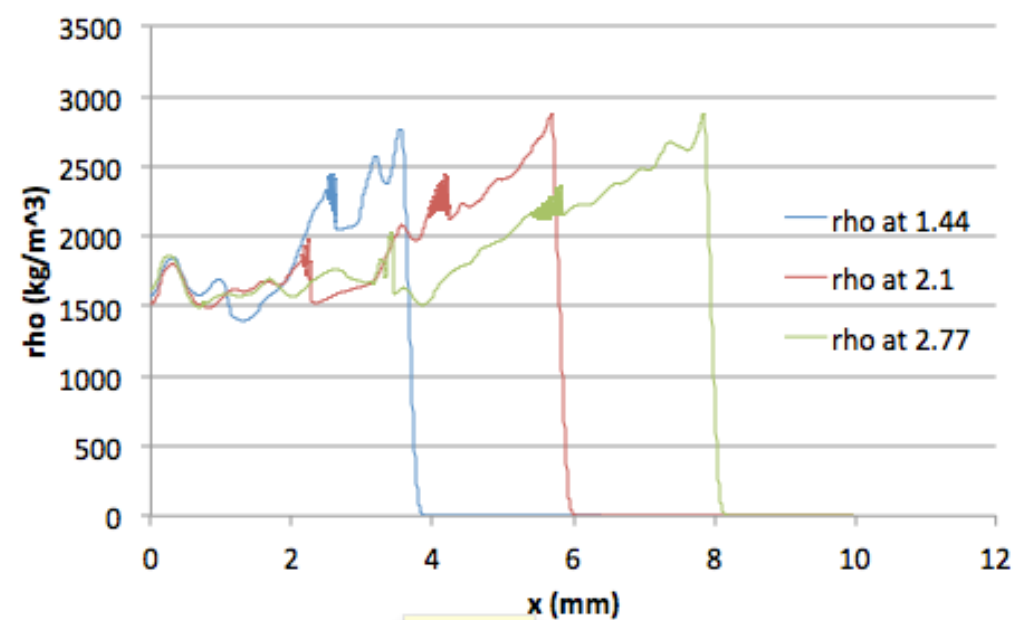

Figure 5.7.1. Gas-phase density at 1.44, 2.1, and 2.77 microseconds. 


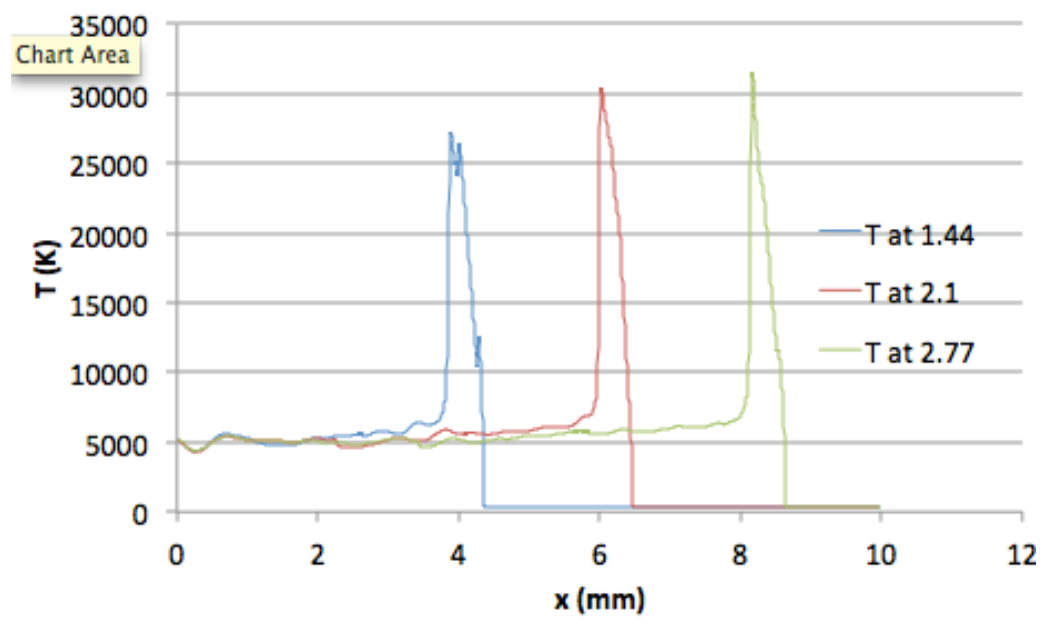

Figure 5.7.2. Fluid velocity at $1.44,2.1$, and 2.77 microseconds.

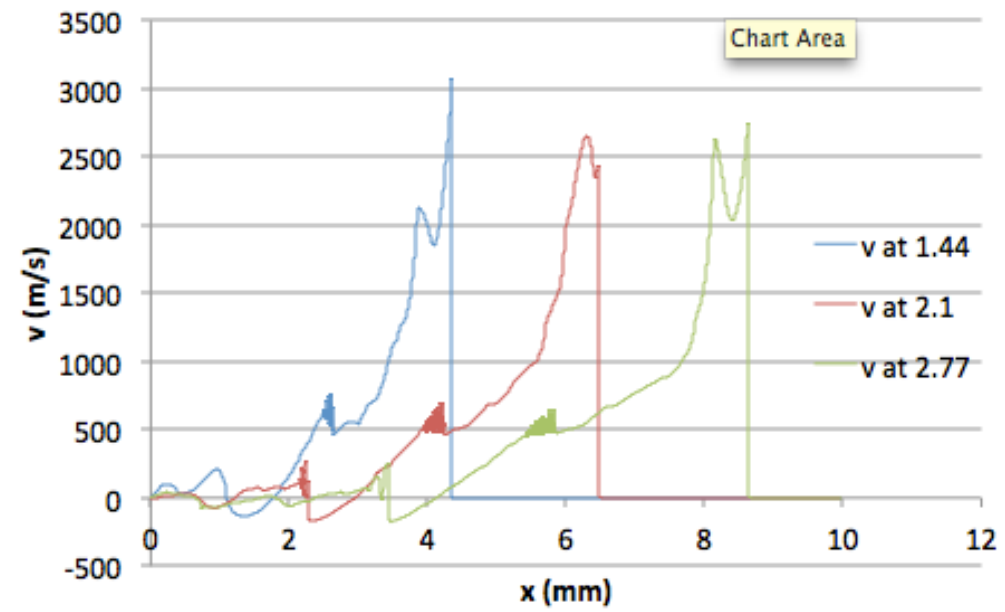

Figure 5.7.3. Gas-phase temperature at 1.44, 2.1, and 2.77 microseconds.

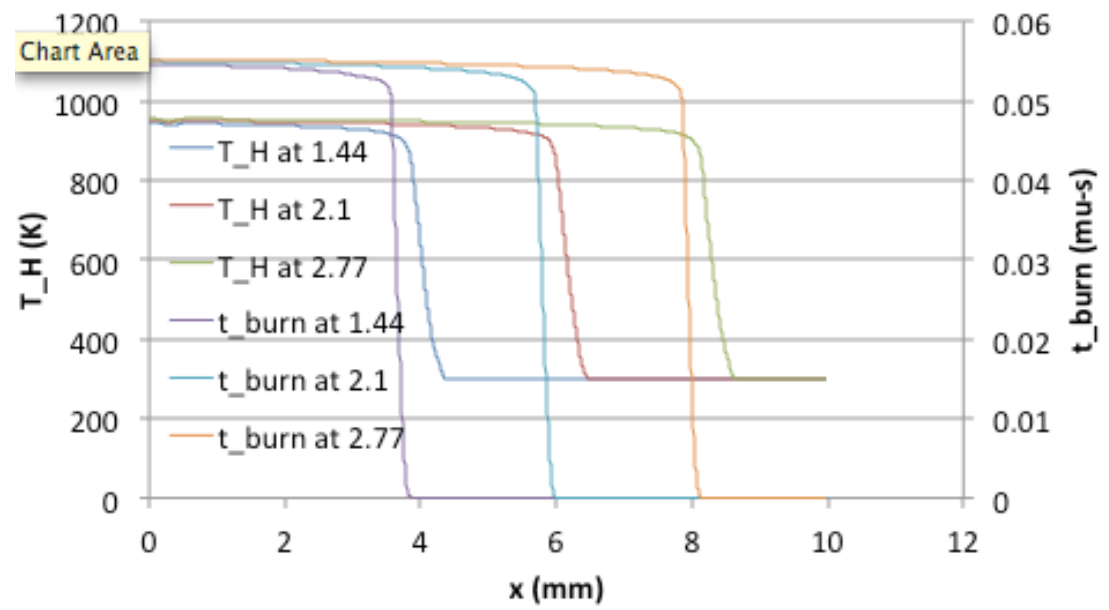

Figure 5.7.4. Solid-phase temperature at 1.44, 2.1, and 2.77 microseconds. Overlaid is the amount of time for which the HE has been burning, showing the spatial shift between the burning region and the heating region ahead of it. 


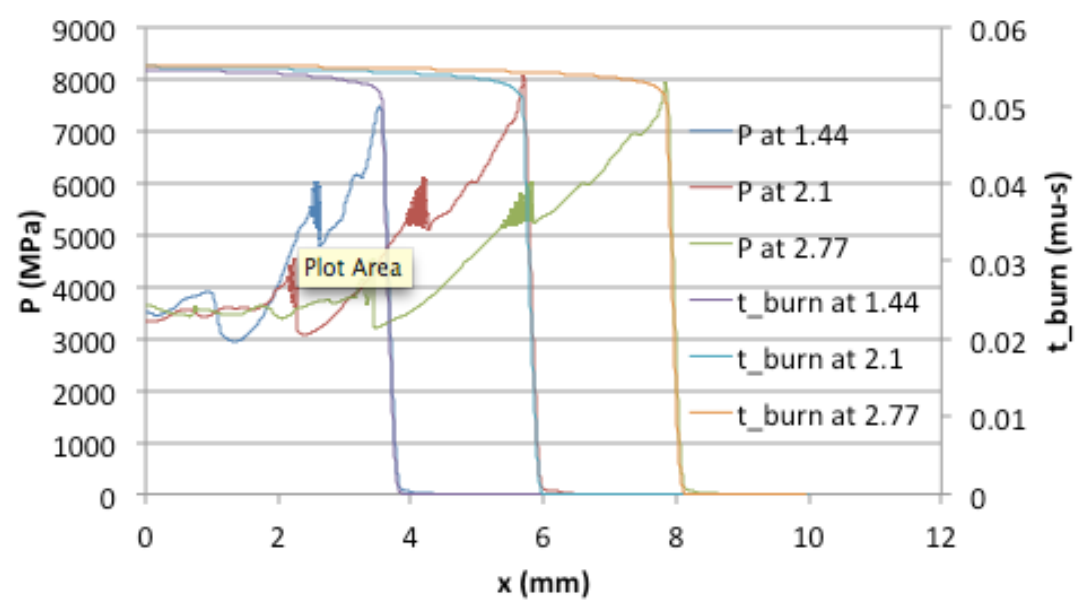

Figure 5.7.5. Gas-phase pressure at 1.44, 2.1, and 2.77 microseconds. Overlaid is the amount of time for which the HE has been burning, showing coincidence with the main pressure rise after burning has already commenced. The initial pressure rise leading to the onset of burn is almost invisible on this scale.

These plots make clear that a steady front has indeed evolved from the initial quiescent state. Two traits of PCB are evident: the front is moving quite fast, supersonic relative to the air in front of it, at about $3.2 \mathrm{~mm} / \mathrm{mu}-\mathrm{s}$. The pressure at the burn front, while significantly elevated above background, is well below shock initiation levels; the burning is driven by the high temperature and not by pressure.

Some other properties of the PCB wave are easier to see if we map the velocities at three different times onto each other in the following way: all points from the upstream end of the burn to the downstream end are shifted proportional to time, as would be expected for a steady-wave structure. All points from the upstream end of the burn to the symmetry plane are scaled linearly so that the end of burn is aligned. What is revealed is a remarkably self-similar structure with relatively small unsteady perturbations added on top. Figure 5.7.6 shows this, along with the solid-phase temperature and the burn time in the burning region; the velocity has been scaled to the unperturbed sound speed and the temperature to the unperturbed value so that they can be displayed on the same scale. The spatial coordinate is plotted in units of $d$, the pore size, to confirm that the salient features are indeed much larger than the grain structure, justifying the continuum approach. 


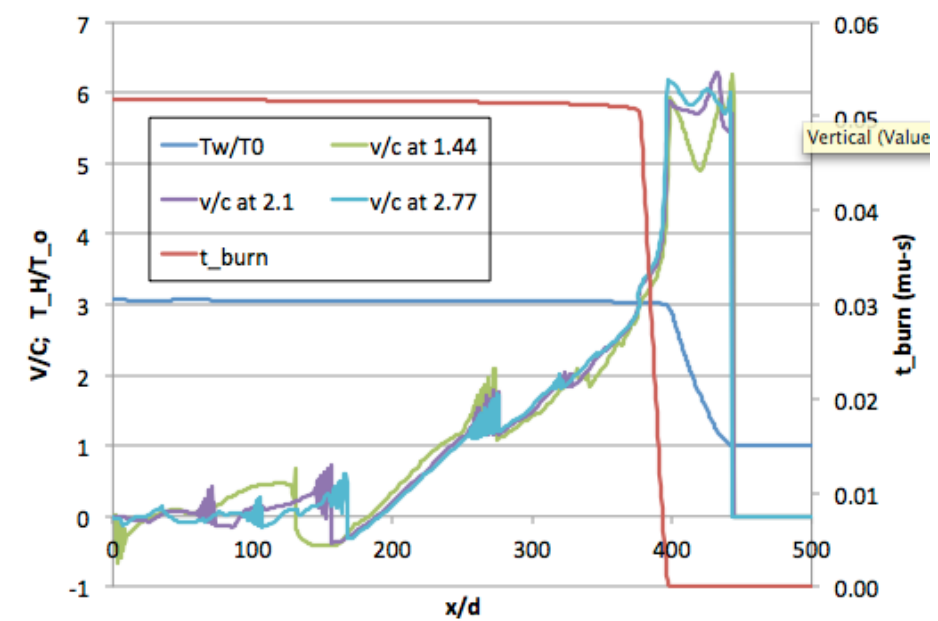

Figure 5.7.6. Overlay of the velocities from 1.44, 2.1, and 2.77 microseconds, where the portion of the curves from the burn region downstream are shifted proportional to time, and the portion upstream is scaled in a self-similar mapping. A solid-phase temperature profile and the burn time at each point are also shown.

With these quantities overlaid, other interesting features can be seen. The shock in the gas phase produces heating in a layer of (idealized) zero thickness, but the solid-phase temperature is continuous (which can easily be proven by considering the jump conditions of the starting model). Moving from downstream to upstream, there is a finite-thickness region where the solidphase heating occurs, until the burn front begins. The shock front and the burn front move at the same constant speed, in spite of the physical separation. Furthermore, since the material velocity upstream of the shock is always less than that of the shock front, and therefore less than the burn front, this implies that, in the frame of the burn front, the convective flow that drives the burn is actually coming in from the non-burning side.

We pinpoint this trait as the defining characteristic of "pumped" convective burn. In a typical convective burn, the hot reaction products flow forward to propagate the burn. Here, shockcompressed air convects backwards through the (non-shock-compressed) solid to propagate the burn.

Conditions upstream of the burn region are subsonic with respect to the fully reacted material. Thus, the wave cannot become isolated from the upstream boundary in finite time and is therefore not truly a steady wave. However, a self-similar character clearly sets in, in a way made somewhat more precise in the mathematical analysis of the next section.

Since the boundary is therefore important, the choice of physical boundary condition will matter. We have chosen a symmetry plane since our context is a spark channel through the HE, which would define a center from which the wave will propagate.

\subsubsection{Details of the Derivation}

As described in the previous section, the PCB wave is "quasi-steady," composed of some approximately steady sections that connect continuously to a self-similar piece. The procedure we follow to analyze the wave is to treat one section at a time, and show that sewing them together requires a particular propagation speed that matches the planar time-dependent 
simulations. Even though it is a slight misnomer, we will refer to these results as the steady-wave solution. With a few reasonable assumptions, it is also possible to derive a completely analytic expression for the wave speed that appears to be good to about $10 \%$.

From the upstream end of the burn region forward (downstream), a steady-wave formulation of the planar model given in the previous section is used. In terms of a steady-wave speed $\mathrm{v}_{\mathrm{s}}$, we change coordinates according to $\mathrm{x}^{\prime}=\mathrm{x}-\mathrm{v}_{\mathrm{s}} \mathrm{t}, \mathrm{t}^{\prime}=\mathrm{t}$, and assume that in this frame there is no variation with respect to t'. Furthermore, we introduce the following normalizations:

$y=x^{\prime} / d$

$\hat{v}=v / v_{s}$

$\hat{\rho}=\rho / \rho_{o}$

$\hat{T}=T / T_{o}$

$\hat{T}_{H}=T_{H} / T_{o}$

$\hat{T}_{\text {crit }}=T_{\text {crit }} / T_{o}$

$\hat{P}=P / P_{o}=\hat{\rho} \hat{T}$

and we introduce an eigenvalue-like quantity involving the wave speed, not quite equal to the square of the Mach number:

$\lambda \equiv \frac{\rho_{o} v_{s}^{2}}{P_{o}}$

We expect $\lambda>>1$. The resulting model is:

$\frac{d}{d y} \hat{\rho}(1-\hat{v})=-\frac{2 \rho_{H} v_{b}}{\rho_{o} v_{s}} H(-y) H\left(y+\frac{d_{H} v_{s}}{2 v_{b} d}\right)$

$\frac{d}{d y}(\hat{P}-\hat{\rho}(1-\hat{v}) \lambda \hat{v})=-\frac{\lambda f \hat{v}^{2}}{2(1-\hat{v})} \hat{\rho}(1-\hat{v}) H(y)$

$\frac{d}{d y}\left((\gamma \hat{v}-1) \frac{\hat{P}}{\gamma-1}-\hat{\rho}(1-\hat{v}) \frac{\lambda \hat{v}^{2}}{2}-U_{o} \hat{T}_{H}\right)=\frac{2 U_{H} v_{b}}{P_{o} v_{s}} H(-y) H\left(y+\frac{d_{H} v_{s}}{2 v_{b} d}\right)$

$\frac{d \hat{T}_{H}}{d y}=\frac{H}{\sqrt{\lambda}}\left(\hat{T}_{H}-\hat{T}\right)$

We have defined the start of the burn region (the downstream end) to be $y=0$. The last equation has been used to move the heat transfer term into the derivative in the gas-phase energy equation; we have not included any special notation to indicate this is only in the region where $y>0$. Finally, we have introduced two dimensionless parameters: 


$$
U_{o} \equiv \frac{\rho_{H} C_{H} T_{o} d_{e f f}}{P_{o} d} \quad H \equiv \sqrt{\frac{\rho_{o}}{P_{o}}} \frac{h d}{\rho_{H} C_{H} d_{e f f}}
$$

In the region ahead of the shock, the conditions are ambient:

$$
\hat{\rho}=\hat{P}=\hat{T}_{H}=1 ; \hat{v}=0
$$

\subsubsection{The Heating Region, $0<\mathrm{y}<\mathrm{y}_{\mathrm{s}}$}

In the heating region between the shock and the onset of burn, the mass and energy source terms are zero, making it easy to integrate the equations from a location $0<\mathrm{y}<\mathrm{y}_{\mathrm{s}}$ to positive infinity. The position of the shock front, $\mathrm{y}_{\mathrm{s}}$, is as yet unknown. The results are:

$$
\begin{aligned}
& \hat{\rho}(1-\hat{v})=1, y>0 \\
& \frac{-1}{\gamma-1}-\frac{\gamma \hat{v}-1}{\gamma-1} \hat{P}+\frac{\lambda \hat{v}^{2}}{2}+U_{o}\left(\hat{T}_{H}-1\right)=0, y>0
\end{aligned}
$$

The momentum equation and solid-phase energy equation take the form:

$$
\begin{aligned}
& \frac{d}{d y}(\hat{P}-\lambda \hat{v})=-\frac{\lambda f \hat{v}^{2}}{2(1-\hat{v})}, 0<y<y_{s} \\
& \frac{d \hat{T}_{H}}{d y}=\frac{H}{\sqrt{\lambda}}\left(\hat{T}_{H}-\hat{P}(1-\hat{v})\right), 0<y<y_{s}
\end{aligned}
$$

On the upstream side of the shock, conditions are no longer ambient. The jump conditions across the shock yield:

$$
\begin{aligned}
& \hat{\rho}_{u p}=\frac{1}{1-\hat{v}_{u p}} \\
& \hat{v}_{u p}=\frac{2}{\gamma+1}(1-\gamma / \lambda) \\
& \hat{P}_{u p}=\frac{\hat{T}_{u p}}{1-\hat{v}_{u p}}=1+\frac{2 \lambda}{\gamma+1}(1-\gamma / \lambda) \\
& \hat{T}_{H, u p}=1
\end{aligned}
$$

We make up for not knowing $\mathrm{y}_{\mathrm{s}}$ by the fact that the system is autonomous in $\mathrm{y}$ and that we have two conditions on the normalized HE temperature: 1 at $\mathrm{y}=\mathrm{y}_{\mathrm{s}}$ and $\mathrm{T}_{\text {crit }}$ at $\mathrm{y}=0$. We therefore switch to $\mathrm{T}_{\mathrm{H}}$ as the independent variable: 


$$
\frac{d}{d \hat{T}_{H}}(\hat{P}-\lambda \hat{v})=\frac{\lambda^{3 / 2} f \hat{v}^{2}}{2 H(1-\hat{v})\left(\hat{T}_{H}-\hat{P}(1-\hat{v})\right)}
$$

From the energy equation we note that:

$$
\frac{d \hat{P}}{d \hat{T}_{H}}=\frac{\partial \hat{P}}{\partial \hat{T}_{H}}+\frac{\partial \hat{P}}{\partial \hat{v}} \frac{d \hat{v}}{d \hat{T}_{H}}
$$

where:

$$
\frac{\partial \hat{P}}{\partial \hat{T}_{H}}=\frac{U_{o}(\gamma-1)}{\gamma \hat{v}-1}, \quad \frac{\partial \hat{P}}{\partial \hat{v}}=\frac{\lambda(\gamma-1) \hat{v}}{\gamma \hat{v}-1}-\frac{\gamma \hat{P}}{\gamma \hat{v}-1}
$$

Thus the final equation is for $\mathrm{v}$ as a function of $\mathrm{T}_{\mathrm{H}}$ :

$\left(\frac{\partial \hat{P}}{\partial \hat{v}}-\lambda\right) \frac{d \hat{v}}{d \hat{T}_{H}}=-\frac{\lambda^{3 / 2} f \hat{v}^{2}}{2 H(1-\hat{v})\left(\hat{T}_{H}-\frac{1-\hat{v}}{\gamma \hat{v}-1}\left((\gamma-1)\left(\frac{\lambda \hat{v}^{2}}{2}+U_{o}\left(\hat{T}_{H}-1\right)\right)-1\right)\right)}-\frac{U_{o}(\gamma-1)}{\gamma \hat{v}-1}$

This equation looks unwieldy but is easily solvable on a spreadsheet or any other ODE-capable tool. The initial condition is $\mathrm{v}=\mathrm{v}_{\text {up }}$ at $\mathrm{T}_{\mathrm{H}}=1$. One can simultaneously integrate:

$$
y_{s}-0=\int_{T_{\text {crit }}}^{1} \frac{\sqrt{\lambda}}{H} \frac{1}{\hat{T}_{H}-\hat{P}(1-\hat{v})} d \hat{T}_{H}
$$

Which gives the thickness of the heating region.

Inspection of these equations reveals some additional information. Since we expect $\lambda>>1$, we note that $\mathrm{v}_{\text {up }} \sim 2 / \gamma+1$. The momentum equation constrains the normalized velocity to be less than 1. In order for the pressure to remain finite everywhere, $\mathrm{v}$ must also be greater than $1 / \gamma$. This is a very narrow window and we might reasonably expect that $\mathrm{v} \sim \mathrm{v}_{\text {up }}$ is a good starting approximation for any term not explicitly involving $1-\mathrm{v}$ or $\gamma \mathrm{v}-1$. Furthermore, since $\mathrm{v}$ is so tightly constrained, we might expect that solving for $\mathrm{dv} / \mathrm{dT}_{\mathrm{H}}=0$ is a means of improving the estimate.

We take exactly this approach, with the further assumption, based on the numerical results, that $\mathrm{P}(1-\mathrm{v})>>\mathrm{T}_{\mathrm{H}}$ across the range of interest. If we define a constant:

$$
K_{o} \equiv \frac{\lambda^{3 / 2} f \hat{v}_{u p}^{2}}{2 H U_{o}(\gamma-1)}
$$

And a function of $\mathrm{T}_{\mathrm{H}}$ : 


$$
K_{1}\left(\hat{T}_{H}\right) \equiv(\gamma-1)\left(\frac{\lambda \hat{v}_{u p}^{2}}{2}+U_{o}\left(\hat{T}_{H}-1\right)\right)-1
$$

The resulting equation is a simple quadratic:

$$
K_{1}\left(\hat{T}_{H}\right)(1-\hat{v})^{2}-K_{o}(\gamma \hat{v}-1)^{2}=0
$$

Whose square root can be taken right away:

$$
\frac{\gamma \hat{v}-1}{1-\hat{v}}=\sqrt{\frac{K_{1}}{K_{o}}}
$$

With solution:

$$
\hat{v}=\frac{1+\sqrt{\frac{K_{1}}{K_{o}}}}{\gamma+\sqrt{\frac{K_{1}}{K_{o}}}}, \quad \hat{P} \approx \frac{K_{1}\left(\hat{T}_{H}\right)}{\gamma \hat{v}-1}
$$

With these simplifications, the expression for the layer thickness becomes:

$$
y_{s} \approx \frac{\sqrt{\lambda}}{H} \int_{1}^{\hat{T}_{c r i t}} \frac{\gamma \hat{v}-1}{1-\hat{v}} \frac{d \hat{T}_{H}}{K_{1}\left(\hat{T}_{H}\right)}
$$

Giving a final answer of:

$y_{s} \approx \frac{\sqrt{\lambda}}{\sqrt{U_{o}(\gamma-1) K_{o}} H} \int_{0}^{\hat{T}_{c r i t}-1} \frac{d f}{\sqrt{\frac{\lambda(\gamma-1) \hat{v}_{u p}^{2} / 2-1}{U_{o}(\gamma-1)}+f}}=\sqrt{\frac{4 \sqrt{\lambda}}{f H U_{o}}}\left(\sqrt{1+\frac{2 U_{o}\left(\hat{T}_{c r i t}-1\right)}{\lambda \hat{v}_{u p}^{2}}}-1\right)$

\subsubsection{The Burning Region, $-\mathrm{d}_{\mathrm{H}} \mathrm{V}_{\mathrm{s}} / 2 \mathrm{v}_{\mathrm{b}} \mathrm{d}<\mathrm{y}<0$}

In our simple constant-rate burn model, once the HE has ignited it becomes a mass and energy source and the solid phase temperature is no longer relevant. In the steady-wave frame, we have defined the burn front to be at $y=0$, and the end of the burn region must take place upstream at at $\mathrm{y}=-\mathrm{y}_{\text {end, }}$, where we define $\mathrm{y}_{\text {end }}=\mathrm{d}_{\mathrm{H}} \mathrm{v}_{\mathrm{s}} / 2 \mathrm{v}_{\mathrm{b}} \mathrm{d}$. In this range, the source terms in the steady-wave mass and energy equations are simple constants and we can integrate those equations, again from a point towards positive infinity, with the source contributions beginning at $y=0$. The results are:

$$
\hat{\rho}(1-\hat{v})=1-R \frac{y}{y_{\text {end }}},-y_{\text {end }}<y<0
$$




$$
\begin{aligned}
& \frac{\lambda(\gamma-1) \hat{v}^{2}}{2}\left(1-R \frac{y}{y_{\text {end }}}\right)-(\gamma \hat{v}-1) \hat{P}+\hat{U}_{o}+\hat{U}_{H} \frac{y}{y_{\text {end }}}=0,-y_{\text {end }}<y<0 \\
& \hat{U}_{o} \equiv(\gamma-1) U_{o}\left(\hat{T}_{\text {crit }}-1\right)-1 ; R \equiv \frac{\rho_{H} d_{H}}{\rho_{o} d} \hat{U}_{H} \equiv(\gamma-1) \frac{U_{H} d_{H}}{P_{o} d}
\end{aligned}
$$

In most applications, we expect $U_{H}$ (hat) to be dominated by the product $\rho_{\mathrm{H}} \mathrm{T}_{\text {react }} / \rho_{\mathrm{o}} \mathrm{T}_{\mathrm{o}}$ buried within it. $U_{o}$ contains $\rho_{\mathrm{H}} \mathrm{d}_{\mathrm{eff}} / \rho_{\mathrm{o}}$ d. This produces a typical hierarchy of:

$$
\hat{U}_{H}>>R>>\hat{U}_{o}>>1
$$

Note that since the original heat transfer term in the energy equation is assumed to drop to zero at $\mathrm{y}=0$, the term involving $\mathrm{T}_{\mathrm{H}}$ is referenced to 1 at infinity and then completes its contribution at $\mathrm{T}_{\mathrm{H}}=\mathrm{T}_{\text {crit. }}$.

In this region, we assume frictional drag is unimportant and so we can also integrate the momentum equation; however, the downstream reference point must by $y=0$. Let us define $v_{i}$ and $P_{i}$ to be the normalized velocity and pressure at $y=0$, which come from Eqs. 5.7.1 with $T_{H}=T_{\text {crit. }}$. Then the steady-wave momentum equation integrates to:

$$
\hat{P}_{i}-\lambda \hat{v}_{i}-\hat{P}+\lambda \hat{v}\left(1-R \frac{y}{y_{\text {end }}}\right)=0
$$

Thus we're left with an algebraic system, which we solve for v. Defining a shorthand:

$$
K_{y}=1-\frac{\gamma\left(\hat{P}_{i}-\lambda \hat{v}_{i}\right)}{\lambda\left(1-R y / y_{\text {end }}\right)}
$$

The final result is:

$$
\hat{v}=\frac{1}{\gamma+1}\left(K_{y}+\sqrt{K_{y}^{2}+\frac{2(\gamma+1)}{\lambda\left(1-R y / y_{\text {end }}\right)}\left(\hat{U}_{H} y / y_{\text {end }}+\hat{U}_{o}+\left(\hat{P}_{i}-\lambda \hat{v}_{i}\right)\right)}\right.
$$

\subsubsection{The Self-Similar region, $\mathrm{y}<-\mathrm{d}_{\mathrm{H}} \underline{\mathrm{V}}_{\mathrm{s}} / 2 \mathrm{v}_{\mathrm{b}} \underline{\mathrm{d}}$}

The self-similar region extends from the symmetry plane out to the upstream end of the burn region. We return momentarily to the time-dependent model and the original $\mathrm{x}$ coordinate. There are no source terms in this region, and we expect continuous behavior, so it will be useful to switch from flux-conservative to the more traditional Eulerian form of the fluid equations:

$$
\frac{\partial \rho}{\partial t}+\frac{\partial}{\partial x} \rho v=0
$$




$$
\begin{aligned}
& \frac{\partial v}{\partial t}+v \frac{\partial v}{\partial x}+\frac{1}{\rho} \frac{\partial P}{\partial x}=0 \\
& \frac{\partial P}{\partial t}+v \frac{\partial P}{\partial x}+\gamma P \frac{\partial v}{\partial x}=0
\end{aligned}
$$

We transform to new coordinates ( $\mathrm{z}, \mathrm{t}$ '), where $\mathrm{z}$ is normalized between 0 and 1 and "stretches" in time so that 1 is always at the interface between the self-similar and steady-wave regions:

$$
z=\frac{x}{x_{i}+v_{s} t}, \quad t^{\prime}=t
$$

The reference size $\mathrm{x}_{\mathrm{i}}$ is assumed to become small relative to $\mathrm{v}_{\mathrm{s}}$ t for any time of interest and will not play a role in what follows. The derivatives transform according to:

$$
\begin{aligned}
\frac{\partial}{\partial t} & =\frac{\partial}{\partial t^{\prime}}-\frac{v_{s} z}{x_{i}+v_{s} t^{\prime}} \frac{\partial}{\partial z} \\
\frac{\partial}{\partial x} & =\frac{1}{x_{i}+v_{s} t^{\prime}} \frac{\partial}{\partial z}
\end{aligned}
$$

Leading to the new system:

$$
\begin{aligned}
& \left(x_{i} / v_{s}+t^{\prime}\right) \frac{\partial \rho}{\partial t^{\prime}}+\left(\frac{v}{v_{s}}-z\right) \frac{\partial \rho}{\partial z}+\frac{\rho}{v_{s}} \frac{\partial v}{\partial z}=0 \\
& \left(x_{i} / v_{s}+t^{\prime}\right) \frac{\partial v}{\partial t^{\prime}}+\left(\frac{v}{v_{s}}-z\right) \frac{\partial v}{\partial z}+\frac{1}{\rho v_{s}} \frac{\partial P}{\partial z}=0 \\
& \left(x_{i} / v_{s}+t^{\prime}\right) \frac{\partial P}{\partial t^{\prime}}+\left(\frac{v}{v_{s}}-z\right) \frac{\partial P}{\partial z}+\frac{\gamma P}{v_{s}} \frac{\partial v}{\partial z}=0
\end{aligned}
$$

It takes an exponentially large number of transit times before one could rigorously claim the system had evolved into a steady flow. However, we observe from the simulations that the transients that are present are not large compared to the emerging steady structure. We further observe that the density and pressure do not vary by large amounts upstream of the completed burn. Therefore, we proceed by assuming a steady self-similar flow and linearizing $\rho$ and $P$ about their values at the interface, $\mathrm{z}=1$, which are the solutions at $\mathrm{y} / \mathrm{y}_{\mathrm{end}}=-1$ from the previous section. We denote the interface values by $\rho_{\text {int }}, v_{\text {int }}$, and $P_{\text {int. }}$. The velocity $\mathrm{v}$ will not be linearized and we normalize it not to the interface value but, consistent with the previous sections, to the front speed $\mathrm{v}_{\mathrm{s}}$ :

$\rho \approx \rho_{\text {int }}\left(1+\hat{\rho}_{1}\right)$ 


$$
\begin{aligned}
& P \approx P_{\text {int }}\left(1+\hat{P}_{1}\right) \\
& (\hat{v}-z) \frac{d \hat{\rho}_{1}}{d z}+\frac{d \hat{v}}{d z}=0 \\
& (\hat{v}-z) \frac{d \hat{v}}{d z}+\frac{\hat{P}_{\text {int }}}{\hat{\rho}_{\text {int }} \lambda} \frac{d \hat{P}_{1}}{d z}=0 \\
& (\hat{v}-z) \frac{d \hat{P}_{1}}{d z}+\gamma \frac{d \hat{v}}{d z}=0
\end{aligned}
$$

The equation for the density is decoupled from the last two. The latter are homogeneous equations that are satisfied either by constant velocity and pressure, or if the determinant can be made to vanish:

$$
(\hat{v}-z)^{2}-\frac{\gamma \hat{P}_{i n t}}{\hat{\rho}_{\text {int }} \lambda}=0
$$

This occurs if:

$$
\hat{v}=z-\sqrt{\frac{\gamma \hat{P}_{i n t}}{\hat{\rho}_{i n t} \lambda}}
$$

where we've chosen the sign to enable achieving $\mathrm{v}=0$ at the boundary, as described below. The other two quantities are given by:

$$
\hat{P}_{1}=\sqrt{\frac{\gamma \hat{\rho}_{\text {int }} \lambda}{\hat{P}_{\text {int }}}}(z-1) \quad \hat{\rho}_{1}=\sqrt{\frac{\hat{\rho}_{\text {int }} \lambda}{\gamma \hat{P}_{\text {int }}}}(z-1)
$$

The unknown steady-wave speed, buried in $\lambda$, is finally revealed by requiring $v=v_{\text {int }}$ at $z=1$ :

$$
\hat{v}_{i n t}=1-\sqrt{\frac{\gamma \hat{P}_{\text {int }}}{\hat{\rho}_{\text {int }} \lambda}}
$$

We discuss this condition shortly. We note that at $\mathrm{z}=\left(\gamma \mathrm{P}_{\mathrm{int}} / \rho_{\mathrm{int}} \lambda\right)^{1 / 2}$ (hatted), $\mathrm{v}$ crosses zero. At this point, we join this solution to the other option, constant velocity and pressure. The solution $\mathrm{v}=0$ is consistent with the boundary condition required by the symmetry axis.

Returning to the pseudo-eigenvalue condition for $\lambda$, let us consider the limit where $U_{H}>>P_{i}$, a reasonable assumption since $\mathrm{P}_{\mathrm{i}}$ is proportional to the internal energy of the gas after shock heating but before any contribution from burning HE, which should be dominant. Based on the parameter hierarchy given earlier, we also expect $\lambda R>P_{i}$. Under these circumstances, 


$$
\begin{aligned}
& \hat{P}_{i n t} \approx \lambda \hat{v}_{i n t} R \\
& \hat{\rho}_{i n t} \approx \frac{R}{1-\hat{v}_{i n t}}
\end{aligned}
$$

which reduces the eigenvalue condition to:

$$
\sqrt{\gamma \hat{v}_{i n t}\left(1-\hat{v}_{i n t}\right)}=1-\hat{v}_{i n t}
$$

with solution:

$$
\hat{v}_{i n t}=1 /(\gamma+1)
$$

Applying the same assumptions regarding $P_{i}$ in the last expression of section 5.7.2.2, we finally arrive at:

$$
\lambda=2(\gamma+1) \hat{U}_{H} / R
$$

which, after unraveling several layers of parameter definitions, boils down to:

$$
\frac{v_{s}}{c_{o}} \approx \sqrt{\frac{2(\gamma+1) T_{\text {react }}}{\gamma T_{o}}}, \quad c_{o}^{2} \equiv \gamma P_{o} / \rho_{o}
$$

Our various scaling assumptions have been born out, and all jump and boundary conditions have been met self-consistently. Thus, a steady-wave solution is proven to exist within the PCB model.

\subsubsection{Discussion of Results}

Figure 5.7.7 below repeats the overlay of Figure 5.7.6, but adds the analytic solution and in the heating region also the steady-wave solution calculated with a spreadsheet. The analytic solution does not capture the boundary layer where the velocity drops from $v_{\text {up }}$ to the equilibrium trajectory, but otherwise captures the shape of the heating region fairly well. The spreadsheet solution captures the boundary layer. Neither solution, of course, can reproduce the superimposed unsteady content seen in the time-dependent simulations. The error in the location of where the self-similar region crosses the $\mathrm{v}=0$ axis may be due to neglect of secondary shocks that appear to be in the numerical results; it may also be due to the fact that those shocks are clearly poorly resolved, with obvious spurious ringing, and thus that the error is in the numerical simulation. 


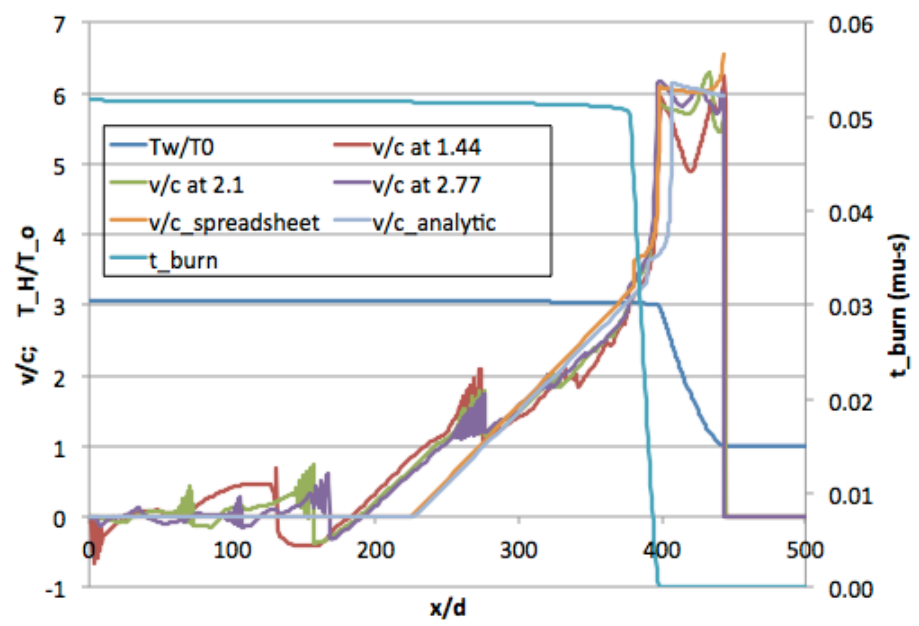

Figure 5.7.7. Overlay of the previous time-dependent solution with the steady-wave solution, shown calculated in the heating region by spreadsheet and also by the stand-alone analytic expressions.

Table 5.7.1 compares the analytic expression for $\mathrm{v}_{\mathrm{S}} / \mathrm{c}$ to results from the time-dependent simulation. The comparison is quite good, matching the dependence on $\mathrm{T}_{\text {react }}$ and confirming the weak dependence on solid-phase density.

\begin{tabular}{|l|l|l|l|}
\hline $\mathbf{T}_{\text {react }}(\mathbf{K})$ & $\boldsymbol{\rho}_{\text {HE }}\left(\mathbf{k g} / \mathbf{m}^{\mathbf{3}}\right)$ & $\mathbf{v}_{\mathbf{s}} / \mathbf{c}$, simulated & $\mathbf{v}_{\mathbf{s}} / \mathbf{c}$, analytic \\
\hline 4500 & 1780 & 7.0 & 7.2 \\
\hline 5000 & 1780 & 7.4 & 7.6 \\
\hline 5500 & 1780 & 7.8 & 7.9 \\
\hline 5500 & 1500 & 7.8 & 7.9 \\
\hline 5500 & 2000 & 8.0 & 7.9 \\
\hline
\end{tabular}

Table 5.7.1. Comparison of analytic and numerical results for the PCB wave speed (normalized to the unperturbed sound speed).

It is noteworthy that the final expression for $\mathrm{v}_{\mathrm{s}}$ is dependent only on the temperature of the reacted $\mathrm{HE}$, without revealing any other conditions required to support a steady wave. These other conditions are buried in the assumptions made at the end regarding the relative magnitude of $\mathrm{P}_{\mathrm{i}}$; these assumptions can be violated by varying either the friction factor $\mathrm{f}$ or the heat transfer coefficient $\mathrm{h}$ (clearly, in the limit of no heat transfer from gas to solid, the solid could never burn). We have not explored these other conditions at this time.

\subsection{Concluding Comments}

Pumped convective burn is a physical model that may provide predictive capability for ESD in low-density HE. So far, the work has shown the following promising signs:

- Threshold predictions of the same order as those observed experimentally-as compared to all prior attempts which were off by orders of magnitude;

- Dependence on parameters such as grain size or specific surface area that at least point in the same direction as those observed experimentally; 
- Power dependence, which has turned out to be difficult to pin down experimentally (to put it mildly);

- A physical picture of fast thermal initiation as something different than transition to shock propagation but nevertheless capable of fast energy release;

- Identification of a new type of steady wave through low-density energetic materials.

Along with the positive, of course, comes a list of things that require further investigation:

- A better model for the ignition and transverse convection at the grain scale

- More robust numerics for Arrhenius kinetics and shock resolution

- Educated selection of correlations for turbulent viscosity and heat transfer

- Is compaction really negligible on this scale?

- Is the fast energy release "fast enough" to drive an adjacent HE (such as a detonator output pellet) into a violent reaction?

And as with all conclusions drawn from a model, experimental confirmation, at a level of detail beyond simple thresholds, will be required.

\subsection{Selected References}

[1] Shajii, A., Freidberg J. P. "Quench in superconducting magnets. I. Model and numerical implementation.” J. Applied Physics vol. 76 \#5, pp 3149-3158, September, 1994.

[2] Shajii, A., Freidberg J. P. "Quench in superconducting magnets. II. Analytic solution." J. Applied Physics vol. 76 \#5, pp 3159-3171, September, 1994.

[3] Shajii, A., Freidberg J. P. "Theory of low Mach number compressible flow in a channel." J. Fluid Mech. vol. 313, pp 131-145, 1996.

[4] Shajii, A. "Theory and modeling of quench in cable-in-conduit superconducting magnets." $\mathrm{Ph}$.D. thesis, Plasma Fusion Center, Massachusetts Institute of Technology, Cambridge, MA, 1994.

[5] Asay, B., Son, S., Bdzil, J. "The role of gas permeation in convective burning." Int. J. Multiphase Flow, vol. 22, \#5, pp. 923-952, 1996.

[6] Ward, M., Son, S., Brewster, M. "Steady deflagration of HMX with simple kinetics: a gas phase chain reaction model." Combustion and Flame, vol. 114, pp. 556-568, 1998.

[7] Asay, B. (Ed.). Non-Shock Initiation of Explosives (Shock Wave Science and Technology Reference Library, Vol. 5). Springer, 2010. ISBN 978-3-540-87952-7.

[8] Tarver, C., Tran, T., Whipple, R. "Thermal decomposition of pentaerythritol tetranitrate." Propellants, Explosives, Pyrotechnics, vol. 28 \#4, pp. 189-193, 2003.

[9] Gifford, M., Luebcke, P., Field, J. "A new mechanism for deflagration to detonation in porous granular explosives.” J. Applied Physics vol. 86 \#3, pp. 1749-1753, August 1999. 
[10] Son, S., Berghout, C., Bolme, A., Chavez, D., Naud, D., Hiskey, M. "Burn Rate Measurements of HMX, TATB, DHT, DAAF, and BTATz." Proceedings of the Combustion Institute, Volume 28, pp. 919-924, 2000.

[11] Penner, S., Williams, F. "The Theory of Steady, One-dimension, Laminar Flame Propagation for One-Step Chemical Reactions.” Astronautica Acta, Vol. 7, pp. 171-189, 1961.

[12] Foltz, M. F. "Pressure Dependence on the Reaction Propagation Rate of PETN at High Pressure." Proceedings of the $10^{\text {th }}$ International Detonation Symposium, Boston, MA, pp. 579-585, July, 1993.

[13] Foltz, M. F. "Pressure Dependence of the Reaction Propagation Rate of TATB at High Pressure." UCRL-JC-108818, October 1991. Also appearing in Propellants, Explosives, Pyrotechnics.

[14] Tarver, C. M., Chidester, S. K., Nichols III, A. L. "Critical Conditions for Impact and Shock Induced Hot Spots in Solid Explosives." J. Physical Chemistry, vol. 100, \#14, pp. 5794-5799, 1996. 


\section{Comparative Response of PETN and TATB: A Preliminary Study}

A rather obvious consequence of arc initiation should not be overlooked: a purely thermal insult can produce a violent HE response, if that insult is sufficiently fast and strong. Moreover, the high level of violence can apparently be reached in a volume that is small compared to a more traditional picture of a "run to detonation."

The failure of the TRIR campaign could be re-interpreted as a positive opportunity to include additional experiments in the LDRD, using the remaining resources. Unfortunately, the PI may have waited a bit too long to abandon TRIR, so that the time remaining was short, but we did decide to begin a preliminary study of the violence of response to arc initiation.

The previous comments about not requiring a run distance can be taken to an obvious limit: an experimental configuration where the HE volume and arc volume are the same. This has two advantages. First, due to limitations on the maximum distance over which the $10 \mathrm{kV}$ pulser could form a discharge through packed powder, the total volume is small and the resulting HE mass ends up well under $10 \mathrm{mg}$, which means experiments could still be easily performed under the B235 R1217 IWS. Second, because we could test powder-filled volumes rather than solid thin films, we could also include TATB (an effort to produce TATB films, outsourced to SNL, never went anywhere). The expectation, of course, is that TATB would retain its usual insensitivity; however, actual tests of this nature have not previously been performed.

A useful application of the test configuration defined below, although not possible with the present pulser design, is to perform threshold testing, since the geometric effects that have caused confusion in ESD testing of larger samples should be minimized.

\subsection{Description of the Configuration}

The test volume is defined by a glass capillary tube of approximately $1 \mathrm{~mm}$ inner diameter. The ends of the volume are set by the end positions of solid copper wire closely match to the tube diameter. The volume is hand-loaded with the powder of interest; then lengths of the copper wire are inserted and epoxied in place. The copper wires are then soldered to copper strips used to provide source and return connections to the pulser. The strips are isolated from each other by sheets of Kapton.

The hand-loading process is difficult to control to high precision, but a typical load was between 1.3 and $1.7 \mathrm{mg}$ of powder (each was weighed after loading), packed to roughly $30 \%$ of theoretical maximum density. The figures below show various views of the assembled tubes. 


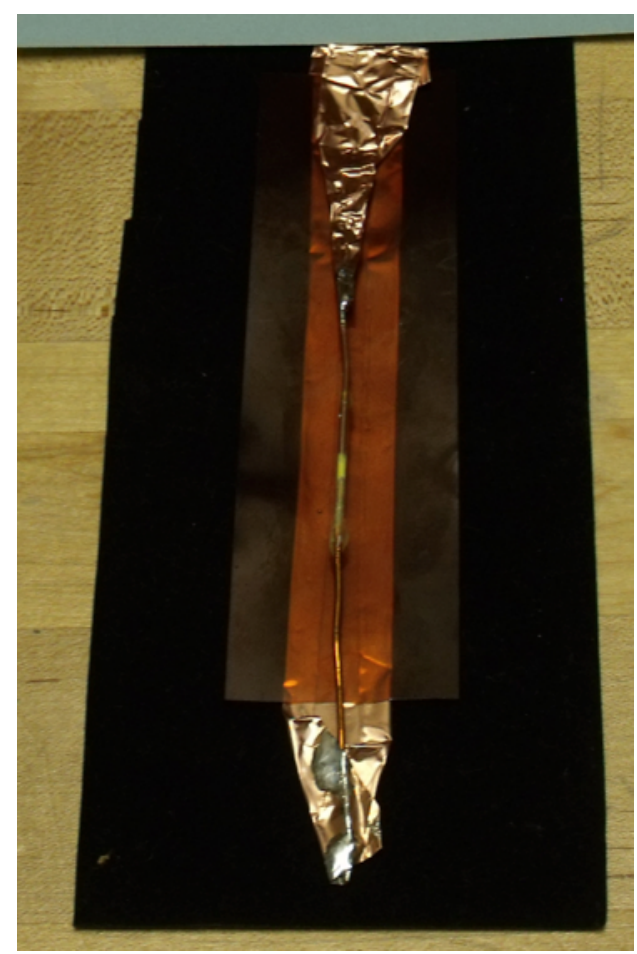

Figure 6.1. View of a complete capillary tube assembly, showing the powder-filled volume at the very center, the copper wire inserted into both ends of the tube, and the copper foil source strip (top) and return (backplane), with Kapton sheet used as insulation.

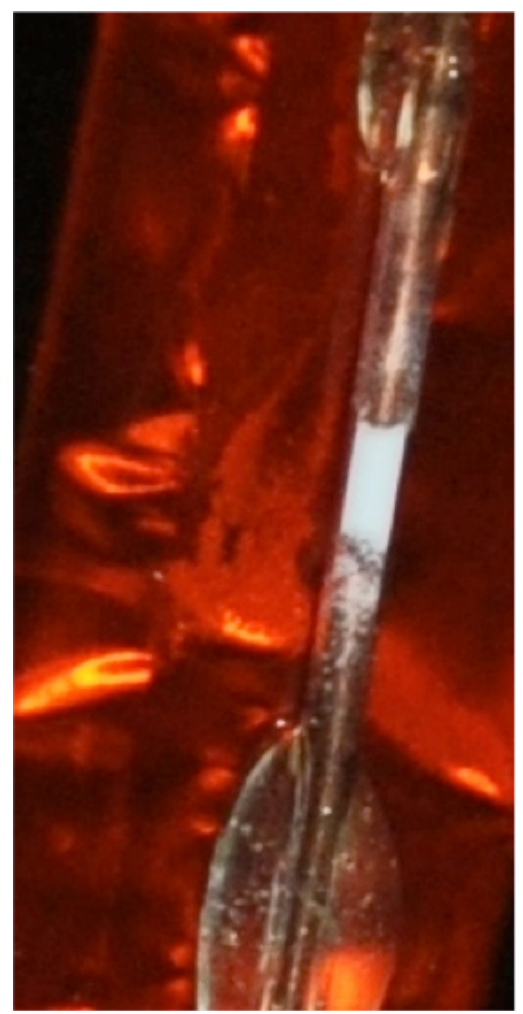

Figure 6.2. Close-up view of the powder-filled volume. The clear blobs at top and bottom are the epoxy used to hold the copper wire in place. 


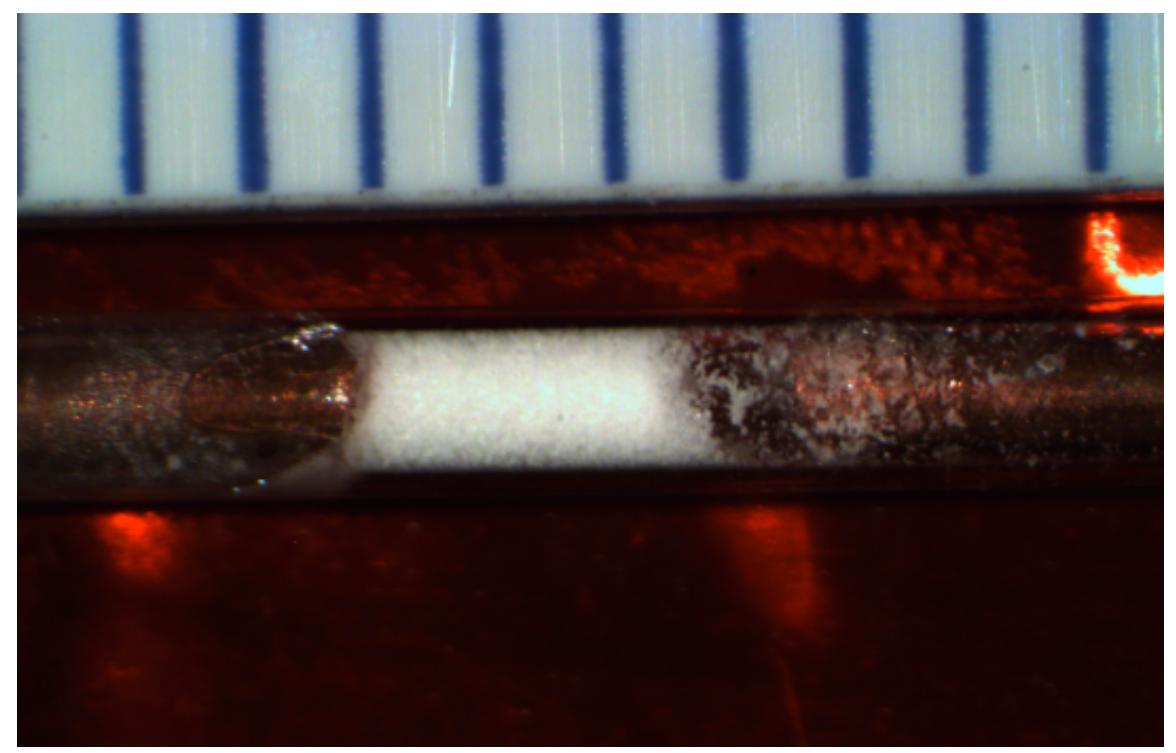

Figure 6.3. View of the powder region with a $1 \mathrm{~mm}$ ruled reference. Typical loads were between 1.3-1.7 mg at $\sim 30 \%$ TMD.

\subsection{Results}

Because of the ad-hoc nature of the assembly, including no easy method for alignment and the lack of a through-hole for line of sight, emission spectroscopy could not be used for these experiments. However, one can easily envision some refinements that would allow the coupling of such a diagnostic. The data acquired for these shots consisted of pre- and post-shot pictures, gated camera images, and video frames taken using the "MIT camera" owned by the HEAF facility. We gratefully acknowledge the assistance provided by Dana Hargrove in the use of the MIT camera.

The test configurations included empty (air-filled) capillaries, and ones loaded with PETN, inert pentaerythritol, and TATB. Empty capillaries typically remained intact following a discharge of the pulser. Capillaries filled with pentaerythritol or TATB would typically crack and also dislocate the copper wire. Much of the powder would burn; some unburned material would be spread locally around the sample arean. Figure 6.4 shows a typical post-shot result of TATB. The behavior of TATB and pentaerythritol, at insult levels of approximately $200 \mathrm{~mJ}$, were visually indistinguishable. 


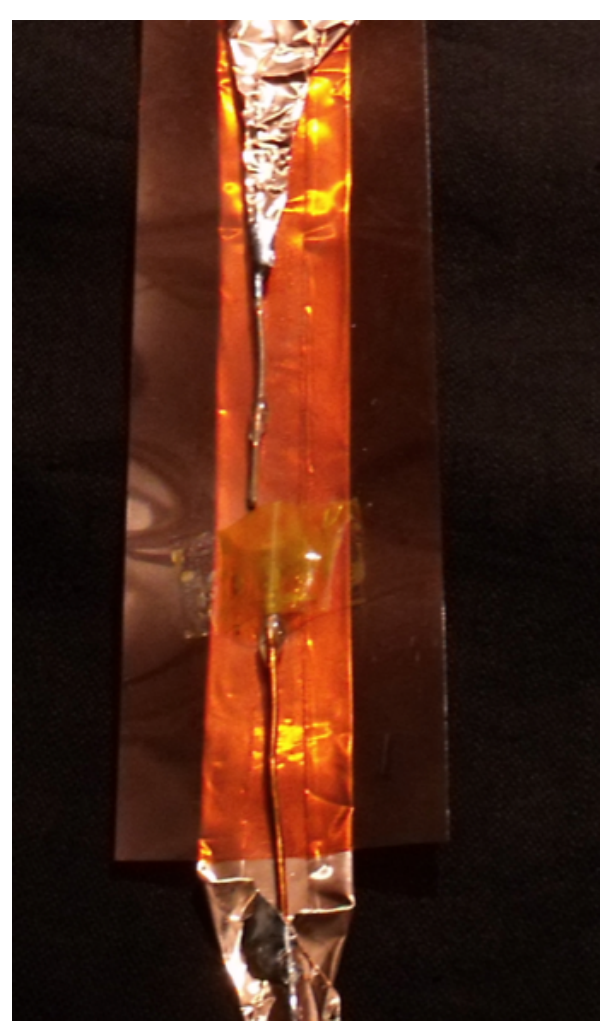

Figure 6.4. Post-shot image of a TATB-filled capillary. The TATB burns non-violently; the resulting pressure cracks the capillary glass and dislocated the copper wire. Inert pentarerythritol behaves similarly.

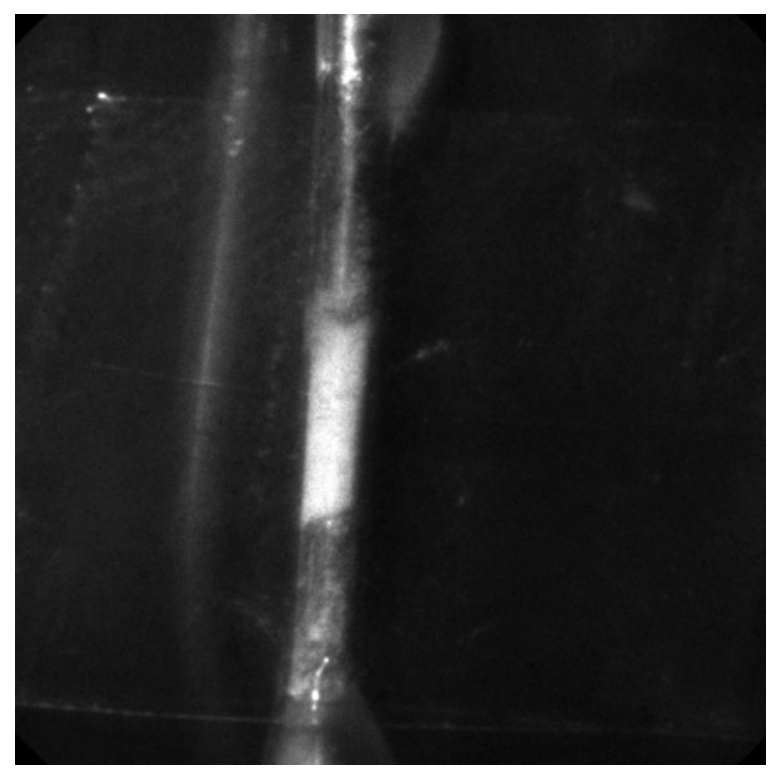

Figure 6.5. Pre-shot view of a TATB capillary, as seen by the gated camera. 


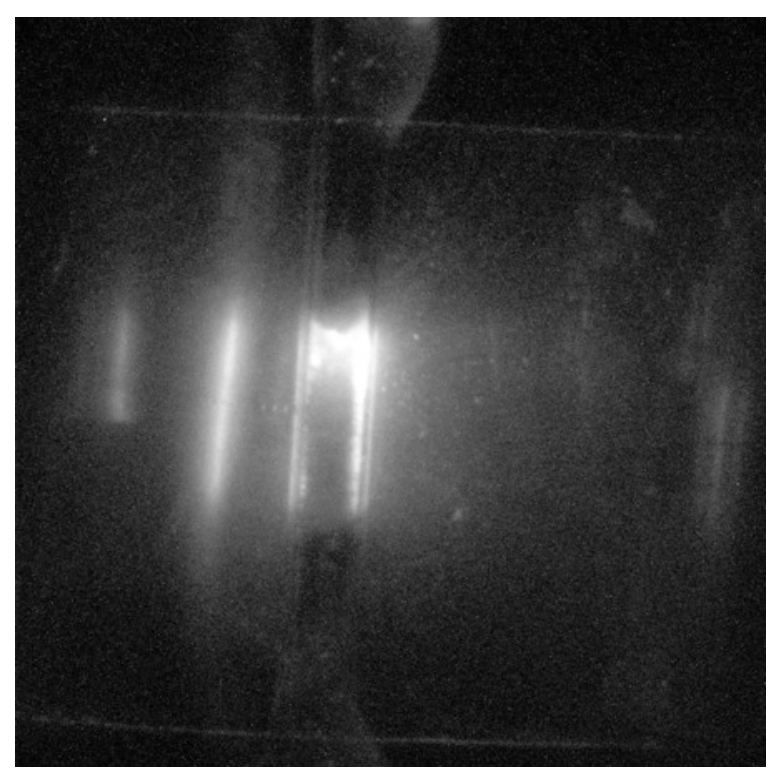

Figure 6.6. Gated image of a TATB capillary taken $1500 \mathrm{~ns}$ into a pulse. The bright region at the top coincides with the slight air gap between the wire and powder as seen in the pre-shot image. The appearance is quite different than PETN imaged at the same time - see Figure 6.8.

The results of testing PETN were dramatically different. The specimen would react with a surprisingly loud pow for $1.5 \mathrm{mg}$. As seen in Figure 6.7, the sample would be completely consumed and the capillary completely destroyed. Enough pressure is produced to deform the copper backplane. The discoloration visible in the figure is not powder residue; it is damaged Kapton. Post-shot analysis using Raman spectroscopy found no trace of unreacted PETN.

Figure 6.8 shows frames from video taken using the MIT camera. The expanding front of the exploding capillary can be tracked and yields an estimate of $1.7 \mathrm{~mm} / \mathrm{microsecond}$ for the speed; this is significantly faster than anything observed in a typical thermal explosion. 


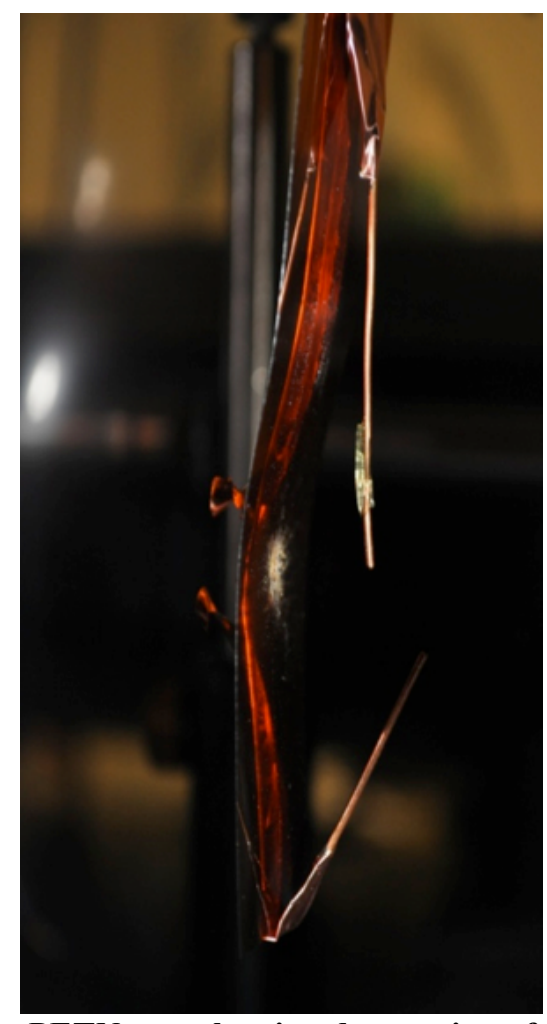

Figure 6.7. Post-shot photograph of a PETN test, showing destruction of the capillary and deformed of the copper backplane. The white blur in the middle is scoured Kapton, not powder residue; Raman spectroscopy confirmed the absence of PETN.
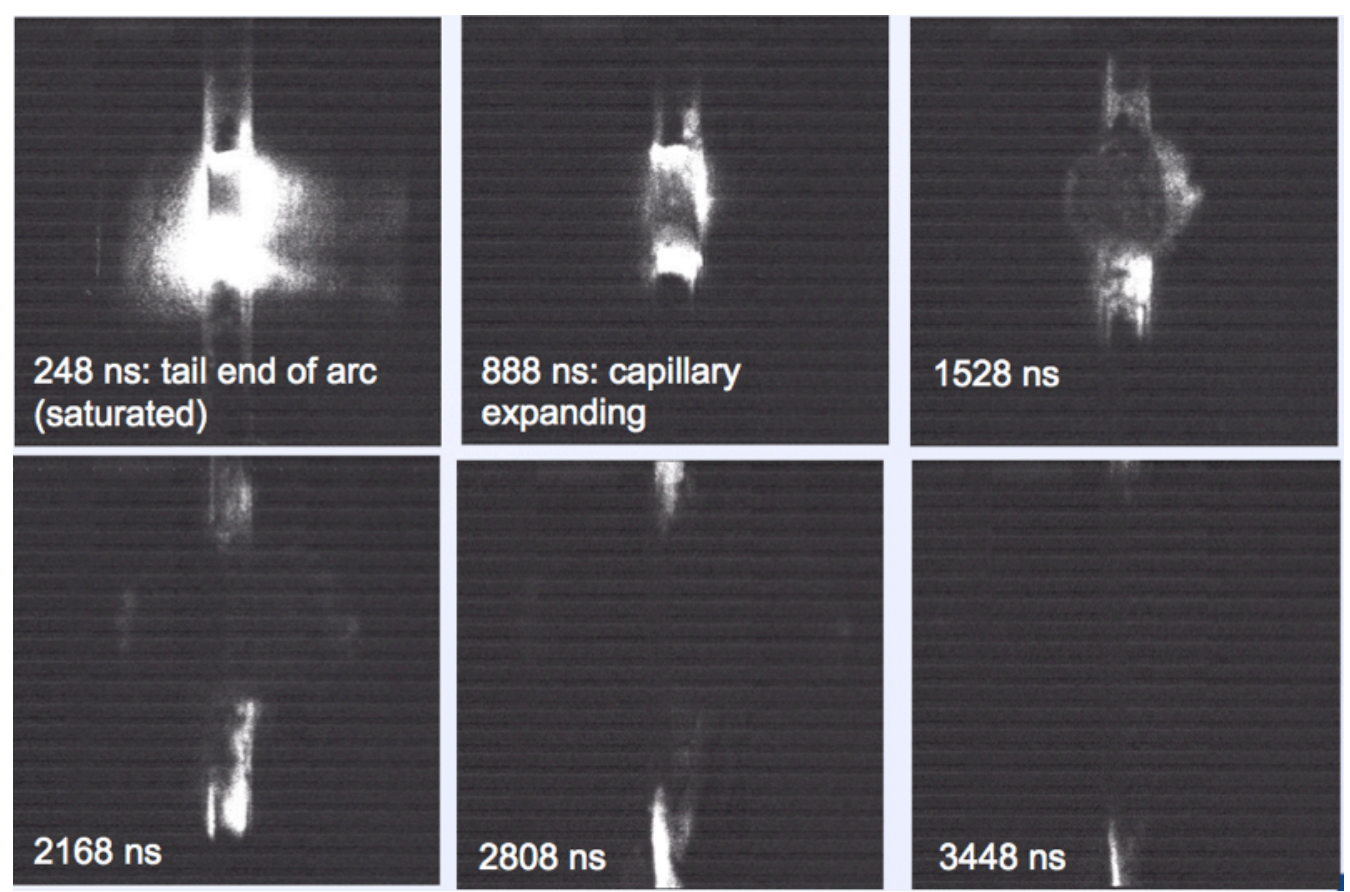

Figure 6.8. Frames from a video of the PETN shot. The expansion speed of the front is estimated to be $1.7 \mathrm{~mm} /$ microsecond. 


\subsection{Recommendations for Future Capillary Testing}

The small footprint of the capillary tests makes them very flexible given the usual limitations on experiments involving high explosives. As this work is transferred to programmatic space, we expect to refine this test by building an improved, standardized holder that can be aligned to within sufficient tolerance to allow use of the imaging spectrometer system. A redesigned pulser that allows tuning of the shot voltage and source energy should be built, to allow true threshold testing and to raise the insult energy to the level of a few joules.

In addition to threshold results, these tests can be used to calibrate a number of assumptions in the PCB model, such as heat transfer. 


\section{Appendix A. Pumped Convective Burn Modeling Tools}

Below is a listing of the $\mathrm{C}++$ tool used to study thresholds according to the pumped convective burn model, in a cylindrical geometry, as discussed in Section 5.5, with the hard-threshold model for $\mathrm{v}_{\text {burn. }}$. The "rungekutta.h" header file contains the interface to a standard Runge-Kutta integration routine. The tool uses an explicit time integration with finite-differencing in space and makes use of a numerical viscosity (representing a normal rather than shear effect), scaling with the square of the velocity gradient, to aid in resolving shocks. The planar-geometry tool used to study the evolution of the steady wave follows this listing.

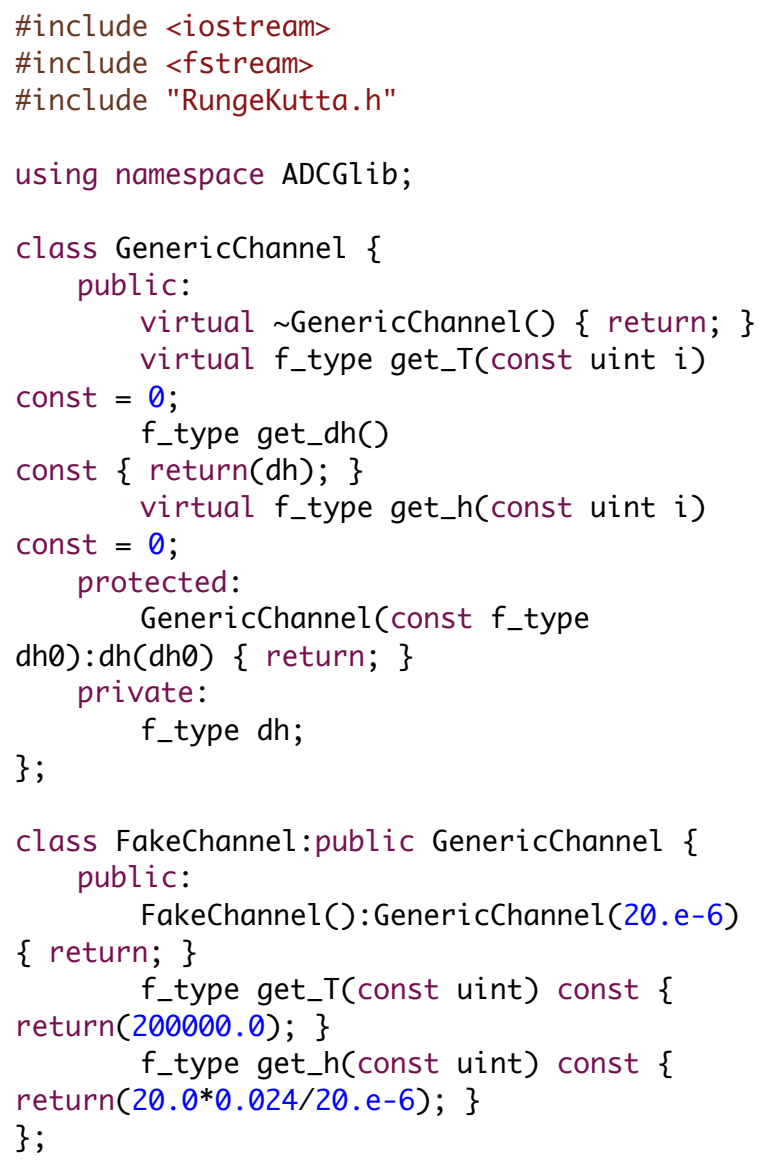

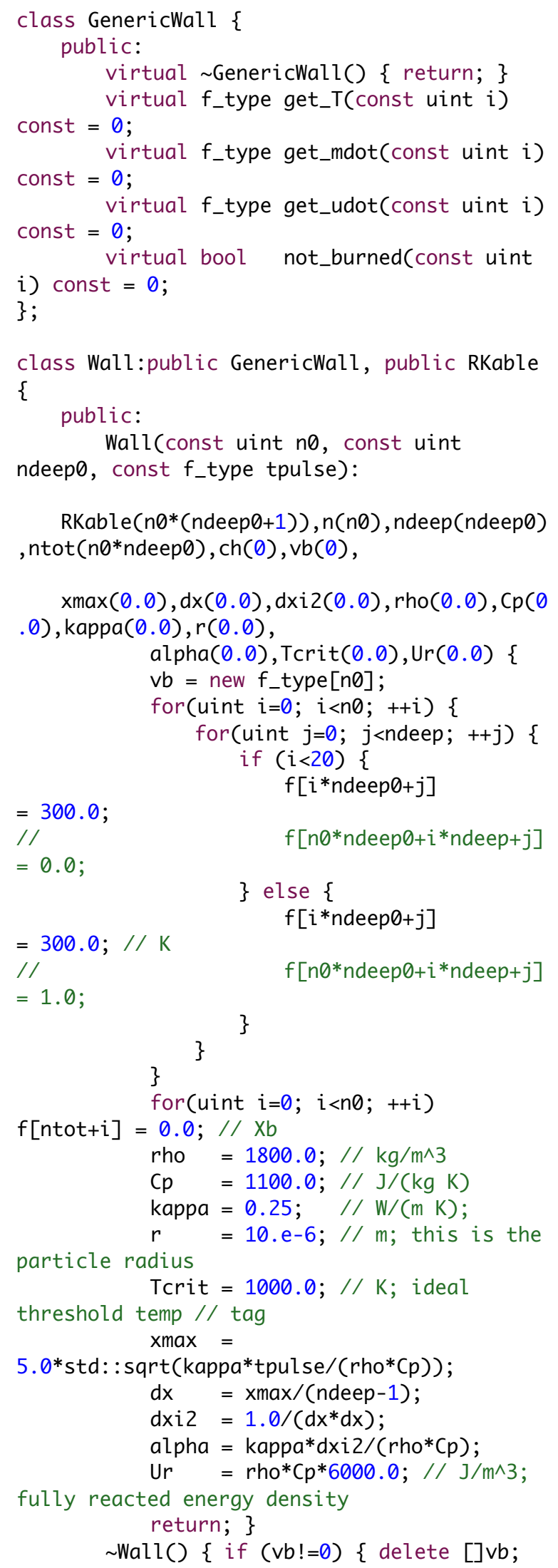


$\mathrm{vb}=0 ;\}$ return; $\}$

void link_channel (const

GenericChannel* ch0) $\{\mathrm{ch}=\mathrm{ch} 0$; return; $\}$

f_type get_T(const uint $i$ )

const $\{$ return $(f[$ ndeep $* i]) ;\}$

f_type get_T(const uint $i$, const uint

$j$ ) const $\{\operatorname{return}(f[$ ndeep $* i+j]) ;\}$

f_type get_mdot (const uint $i$ )

const \{ return $\left(2.0^{*} \mathrm{vb}[\mathrm{i}] *\right.$ rho/ch->get_dh( )); \}

f_type get_udot (const uint i)

const $\left\{\right.$ return $\left(2.0^{*} \mathrm{vb}[\mathrm{i}] * \mathrm{Ur} / \mathrm{ch}->\right.$ get_dh( )); \}

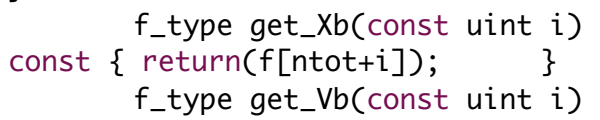

const $\{\operatorname{return}(\mathrm{vb}[\mathrm{i}]) ; \quad\}$

f_type get_y(const uint i)

const $\left\{\operatorname{return}\left(i^{*} d x\right) ;\right.$ \}

bool not_burned(const uint i)

const $\{$ return $(f[$ ntot $+i]<r) ;\}$

f_type get_f(const uint $i)$

const $\{\operatorname{return}(f[n *$ ndeep $+i]) ;\}$

const \{

f_type get_f_front(const uint i)

uint $j=0$;

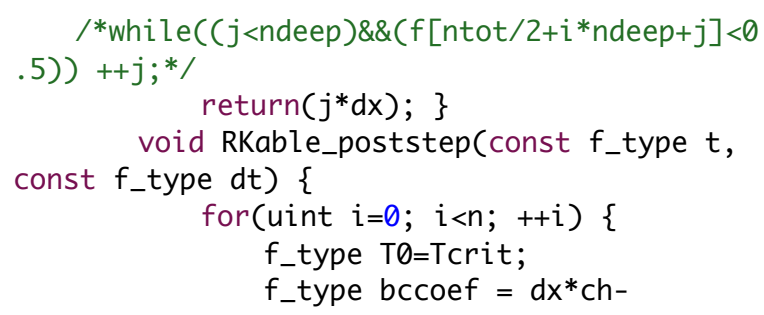

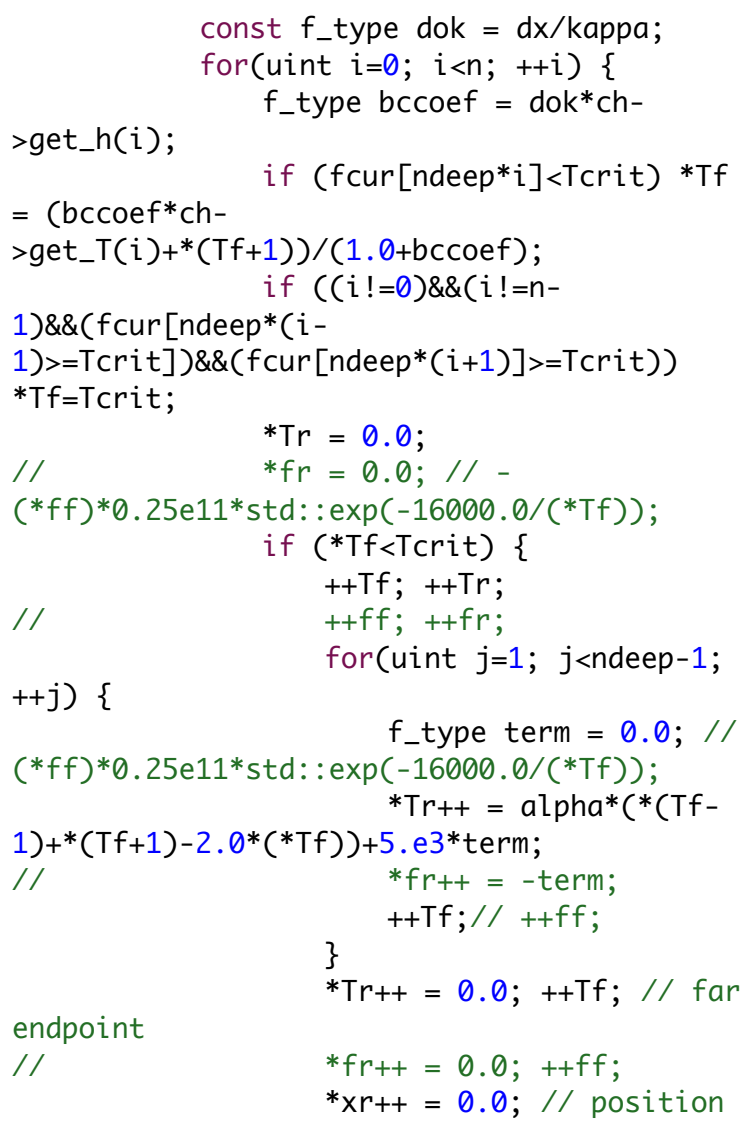

class Channel:public GenericChannel, public RKable \{ 
GenericChannel(20.e-

6), RKable( $3 * n \theta), w(\theta), n(n \theta)$, tpulse(tpo),

$d x(0.0), d x i(0.0), d x i 2(0.0), L(0.0), \operatorname{TpU}(0.0$

)$, \operatorname{TpP}(0.0), \operatorname{mua}(0.0), \operatorname{vpeak}(\theta .0), \operatorname{Xf}(0.0)\{$

$\mathrm{L} \quad=0.0025$

$\mathrm{dx}=\mathrm{L} /(\mathrm{n} \theta-1)$

$d x i=0.5 / d x$;

$d x i 2=4.0 * d x i * d x i$

$\mathrm{TpP}=28.0 * 1.67 \mathrm{e}-27 / 1.38 \mathrm{e}-23$;

$\mathrm{TpU}=0.4 * \mathrm{TpP}$

mua $=d x i * d x i * 5 . e-9 ;$

for (uint $i=0 ; i<n \theta ;++i)\{$

$f[R H O * n \theta+i]=1.2$;

$f\left[\mathrm{GAM}^{*} n \theta+i\right]=0.0$;

$f\left[E^{*} n \theta+i\right]=$

$(300.0 * 1.2 / \mathrm{TpU})$;

\}

return; \}

void link_wall(const GenericWall* w0)

$\{\mathrm{w}=\mathrm{w} 0 ;$ return; $\}$

f_type S(f_type $x$, const f_type $t$ )

const \{

if ( $t<$ tpulse) \{

const f_type $r 0=125 . e-6$;

const f_type $S 0=0.05 /(2.5 \mathrm{e}-$

$3 * 3.14159 * r 0 * r 0) ; / / 73 \mathrm{~g}$ at $d=10,80 \mathrm{~g}$ at

$d=20$, all for $r=250 \mathrm{mu}$

$$
x /=r \theta \text {; }
$$

return $\left(S 0 * \exp \left(-\mathrm{X}^{*} \mathrm{x}\right) /\right.$ tpulse $)$;

\} else return( 0.0$) ;$ \}

f_type get_T(const uint $i$ ) const \{

f_type $U=$ get_U(i);

return $(\mathrm{TpU} * \mathrm{U} / \mathrm{f}[\mathrm{i}]) ;\}$

f_type get_rho(const uint i) const \{ $\operatorname{return}(\mathrm{f}[\mathrm{i}])$; $\}$

f_type get_gam (const uint i) const \{ $\operatorname{return}(f[n+i]) ; \quad\}$

f_type get_E( const uint $i)$ const \{ $\operatorname{return}(f[2 * n+i]) ; \quad\}$

f_type get_v( const uint $i)$ const \{ $\operatorname{return}(f[n+i] / f[i]) ;\}$

f_type get_U( const uint i) const \{ $\operatorname{return}(f[2 * n+i]-0.5 * f[n+i] * f[n+i] / f[i]) ;\}$

f_type get_P( const uint $i)$ const \{ $\operatorname{return}(0.4 *$ get_U(i)); \}

f_type get_r( const uint $i)$ const \{ $\operatorname{return}(i * d x)$; \}

f_type get_vpeak() const \{

$\operatorname{return}(\mathrm{vpeak}) ; \quad\}$

f_type get_Xfront() const \{

$\operatorname{return}(\mathrm{Xf})$;

f_type get_h(const uint i) const \{

// Dittus-Boelter but assumes $\mathrm{Pr}=1$

f_type kappa $=0.024$;

if $(\mathrm{f}[\mathrm{i}]>1.2)$ kappa $+=$

$0.226 *(f[i]-1.2) / 1800.0 ; / /$ crude attempt to mix gas \& HE values

$$
\text { f_type mu }=1.8 \mathrm{e}-5^{*} \text { kappa/0.024; }
$$

// similar crude scaling of mixture

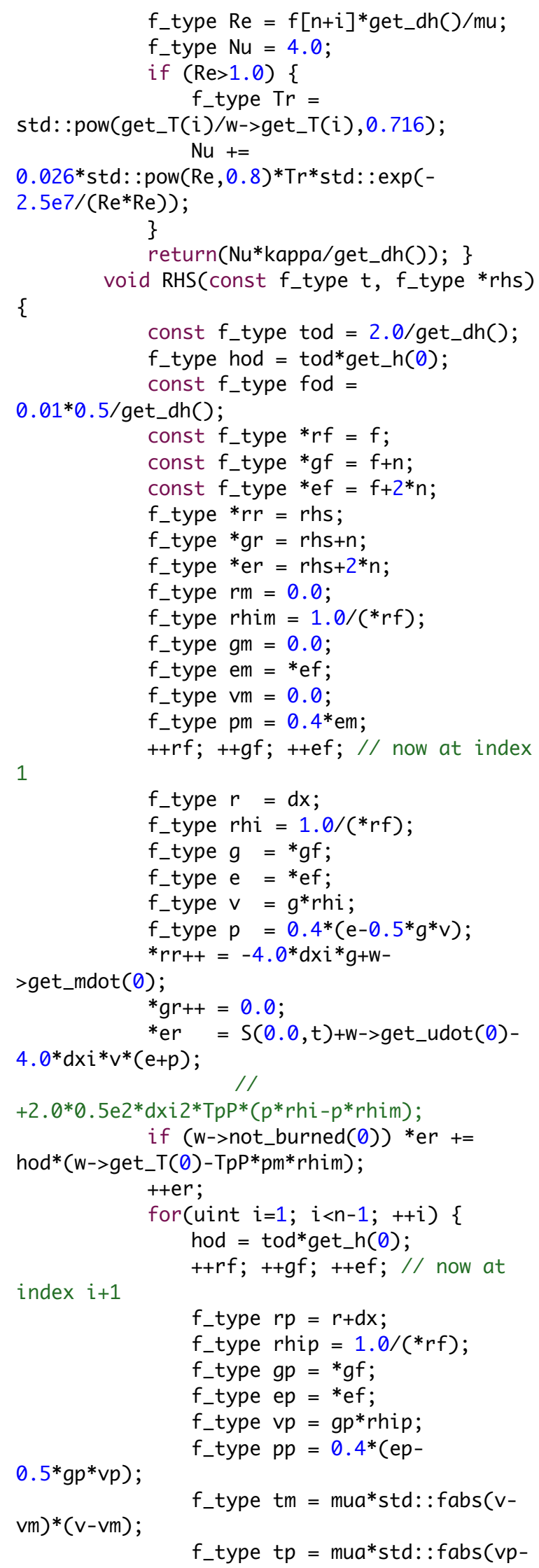




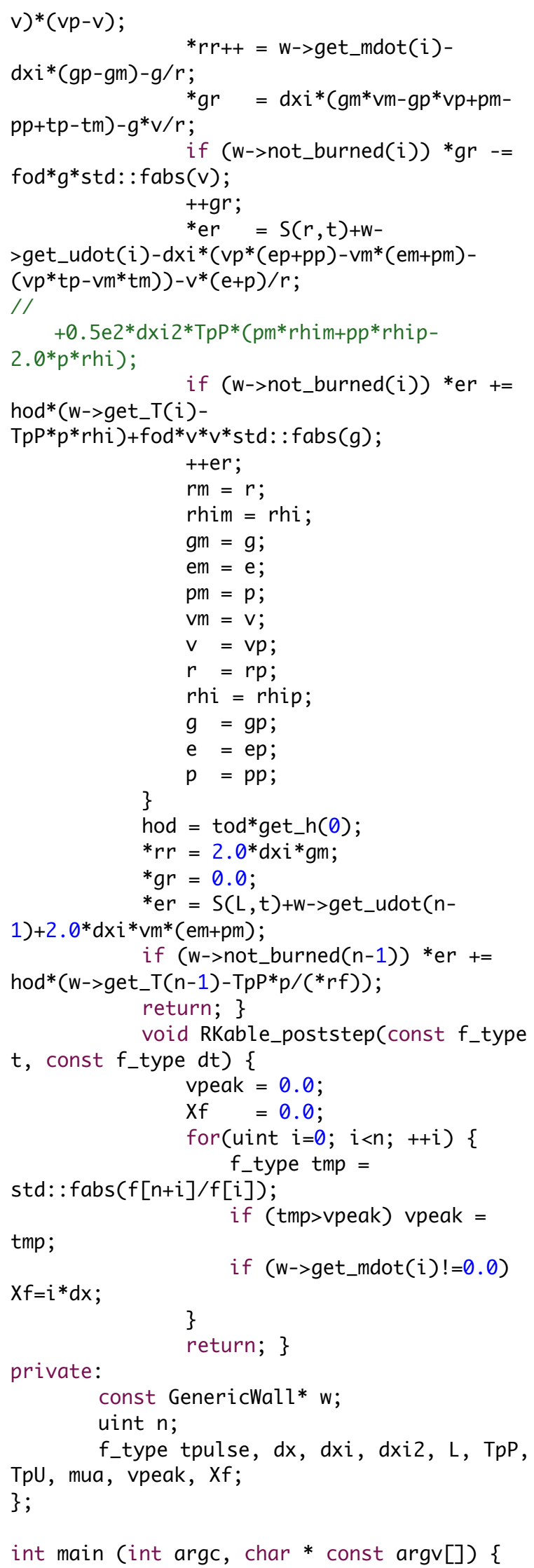




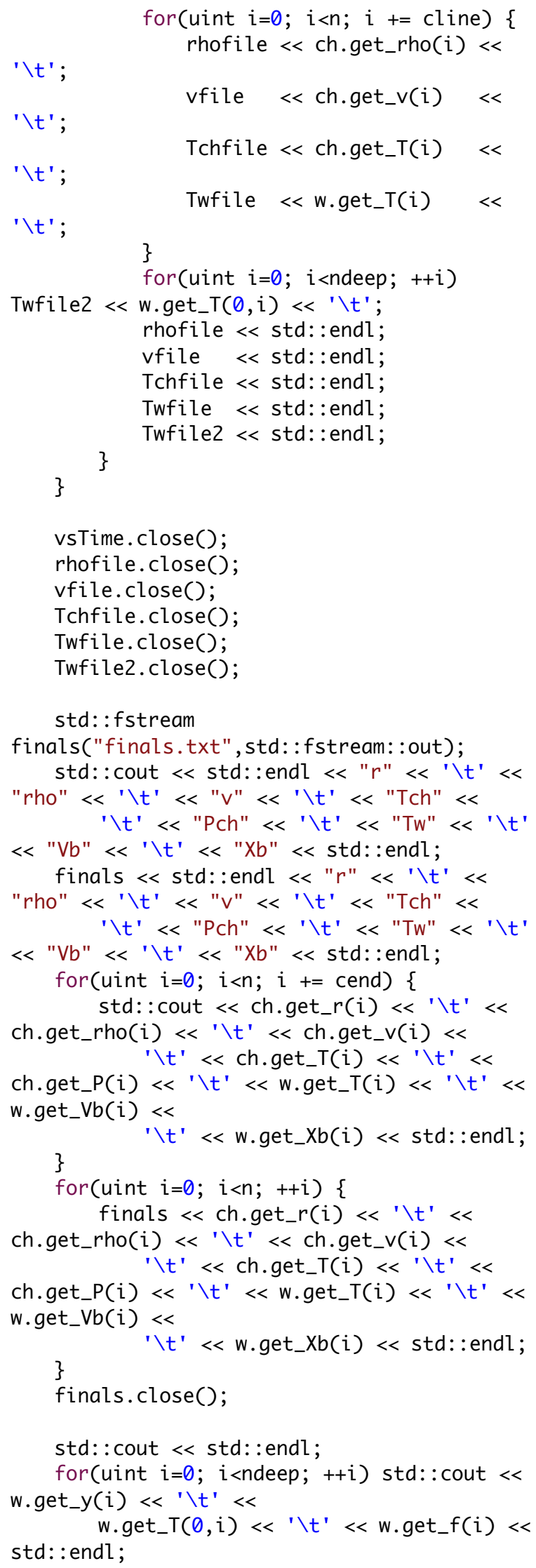


$\left(0.015 /\left(t_{\text {_ppulse }} * 3.14 * 2.5 \mathrm{e}-3 * 6.25 \mathrm{e}-8\right)\right) *(1 . \mathrm{e}-$ $5 /(P 0 * C))$;

$g m 1=0.4$

tcut $=$ t_pulse*c/1.e-5;

$\operatorname{tmax}=(0.5 * 1 . e-5 / v b) *(c / 1 . e-$

5);

Tcrit $=3.0$

argt $=220.0 /$ tmax; $/ /$ for use

in smoothing

$$
\begin{aligned}
& \text { if (filename }==0)\{ \\
& \text { for }(\text { uint } i=0 ; i<n \theta ;
\end{aligned}
$$

++ i) \{

//Planar

1.0

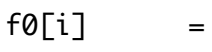

// Cylindrical

//

$\mathrm{i}^{*}$ dy

/I

$\mathrm{y}$;

0.0

// Planar

1.0/gm1;

// Cylindrical

//

$\mathrm{y} / \mathrm{gm} 1$;

1.0

0.0

f_type $y=$

$\mathrm{fO}[\mathrm{i}]=$

$f 0[n \theta+i]=$

$f 0[2 * n \theta+i]=$

$f 0[2 * n 0+i]=$

$f 0[3 * n \theta+i]=$

$f 0[4 * n \theta+i]=$

$$
\} \text { else } \stackrel{\{}{\{} \text { std: :fstream }
$$

file(filename, std: : ios_base: :in);

++ i) \{

$$
\text { for(uint } i=0 ; i<n \theta \text {; }
$$

file $>$ fO[i];

file $>>$

$f 0[n \theta+i]$

$f 0[2 * n \theta+i]$;

$f 0[3 * n \theta+i]$;

file >>

file >>

file >>

$f 0[4 * n \theta+i] ;$

$$
\begin{aligned}
& \text { \} file.close }() ; \\
& \text { return; }\} \\
& \text { Stretch() }\{ \\
& \text { if }(\mathrm{fO} \quad !=0)\{\text { delete }[] \mathrm{fO} ;
\end{aligned}
$$

fo $=0 ;\}$ return; \}

f_type y( const uint i) const $\{\operatorname{return}(d y * i) ;\}$

// Planar

f_type rho(const uint i) const $\{\operatorname{return}(f O[i]) ;\}$ $f_{\text {_type }} P($ const uint $i)$ const $\{\operatorname{return}(g m 1 *(f O[2 * n+i]$ -

$0.5 * f 0[n+i] * v(i))) ;\}$

f_type v( const uint $i)$ const $\{\operatorname{return}(f 0[n+i] / f O[i]) ;\}$

f_type $T($ const uint $i)$ const

$\{\operatorname{return}(\mathrm{P}(\mathrm{i}) / \mathrm{fO}[\mathrm{i}]) ;\}$

f_type gam(const uint $i$ ) const

$\{\operatorname{return}(\mathrm{fO}[\mathrm{n}+\mathrm{i}]) ; \quad\}$

f_type $E($ const uint i) const

$\left\{\operatorname{return}\left(f O\left[2^{*} n+i\right]\right) ;\right\}$

f_type Tw( const uint i) const

$\left\{\operatorname{return}\left(\mathrm{fO}\left[3^{*} \mathrm{n}+\mathrm{i}\right]\right) ;\right\}$

f_type tau_to_t(const f_type tau)

const $\left\{\operatorname{return}\left(\mathrm{tau} /\left(1 . \mathrm{e} 7^{*}\right.\right.\right.$ tcut $\left.\left.)\right) ;\right\}$

f_type tb(const uint $i$ ) const

$\{\operatorname{return}(f 0[4 * n+i]) ;\}$

f_type $x \max ()$ const \{

uint imax $=n-1$;

f_type $\operatorname{vmax}=0.0$;

1];

f_type $v p=f 0[2 * n-1] / f 0[n-$

for(uint $i=n-1 ; i !=0 ;--i)\{$

$f 0[n+i] / f 0[i]$

$=v-v p ; \quad i \max =i ;\}$

$$
\text { f_type } v=
$$

if $(v-v p>v \max )\{\operatorname{vmax}$

\}

$\mathrm{vp}=\mathrm{v}$

return (dy*imax); $\}$

f_type Qext(const f_type y, const

f_type t) \{

$5 / 250 \cdot e-6$

$\left.\left.r^{*} r\right)\right)$

if $(t<t$ cut $)\{$

f_type $r=y^{*} 1 . e-$

return $(Q 0 *$ std: : $\exp (-$

\} else return(0.0); \}

void step(const f_type $t$, const

f_type dt) \{RungeKutta: $\operatorname{RKstep}(t, d t, f 0, f 0)$; return; \}

\{

f_type smooth(const f_type arg) const

if $(\arg <-23.0)$ return(0.0);

if $(\arg <0.0)$

return(0.5*std: : $\exp (\arg ))$;

$0.5 *$ std: : $\exp (-\arg ))$;

if $(\arg <23.0)$ return(1.0-

return(1.0); \}

void RHS(const f_type $t$, const

f_type $* f, f_{-t y p e *}$ rhs $)\{$

f_type vm, v;

f_type Pm, P;

f_type $T m, T, T w=f\left[3^{*} n\right]$;

f_type $r m i=1.0 / f[0]$,

$r i=1.0 / f[1]$

f_type $g m=f[n], \quad g=f[n+1]$;

f_type $E m=f[2 * n], E=f[2 * n+1]$;

f_type $s r=0.0$;

f_type su $=0.0$; 


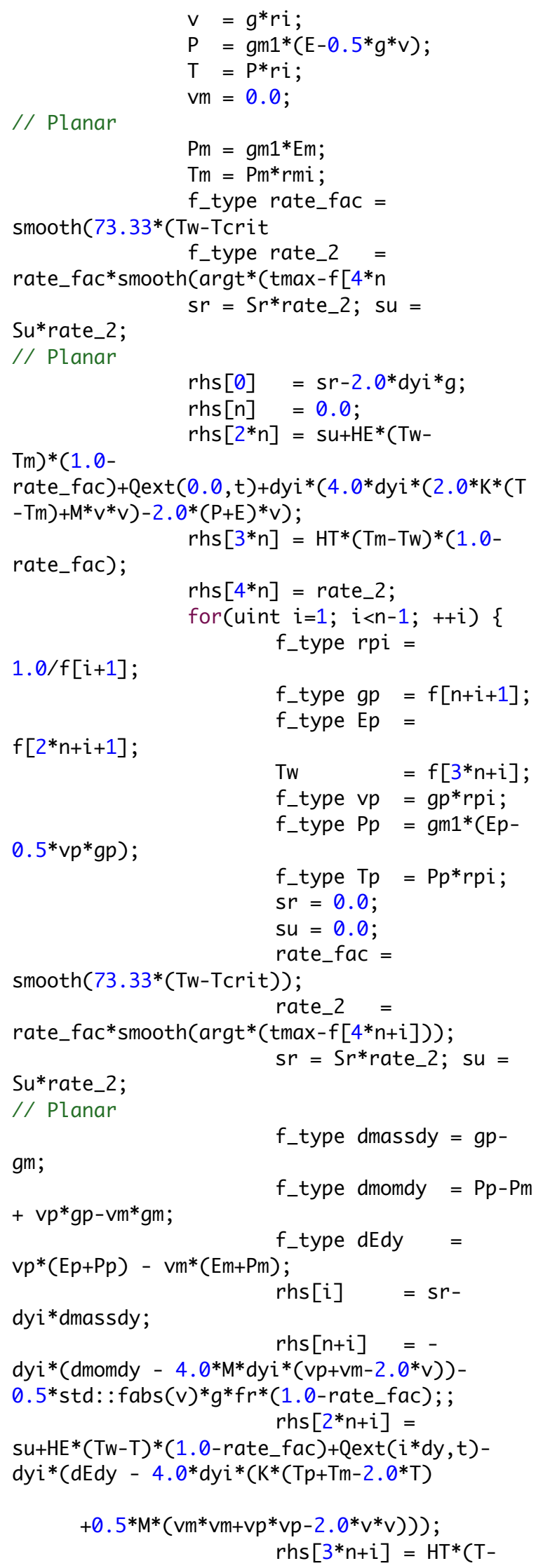

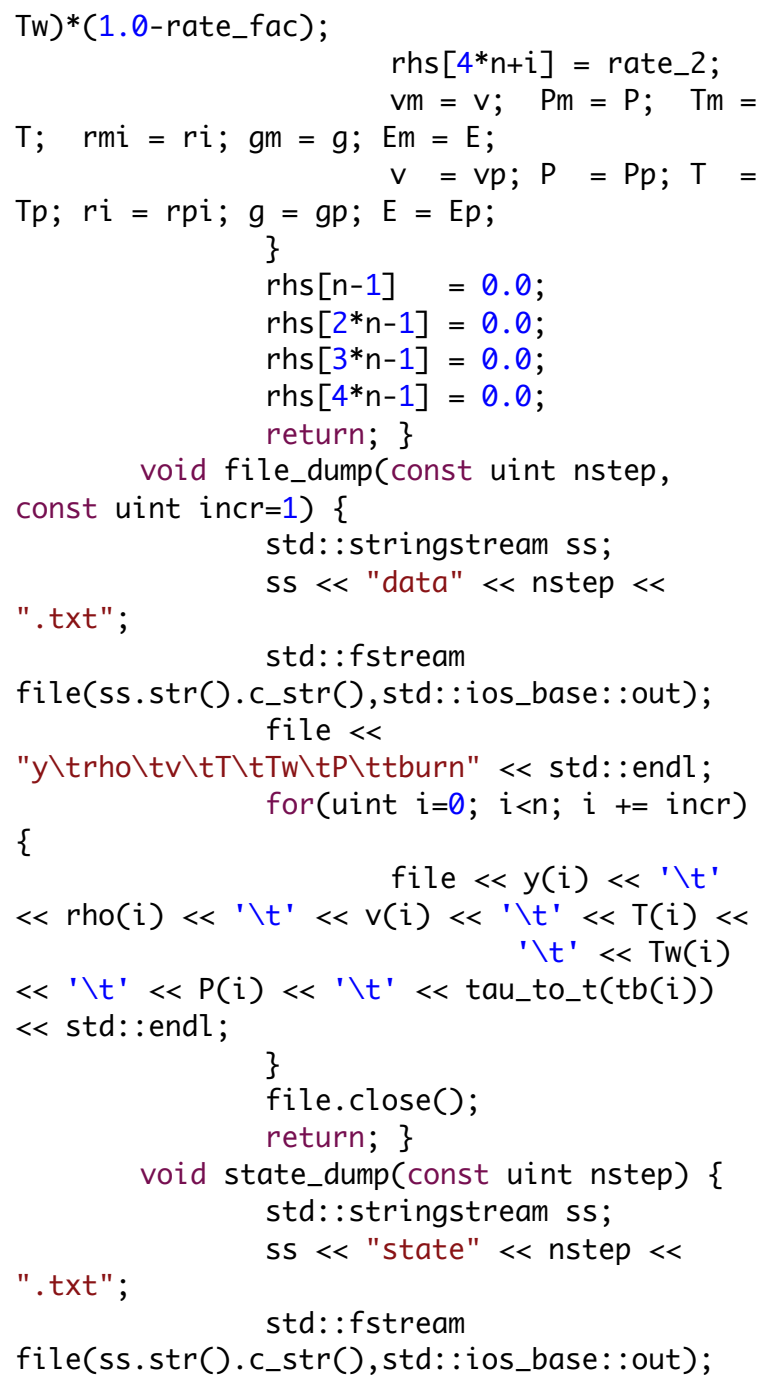




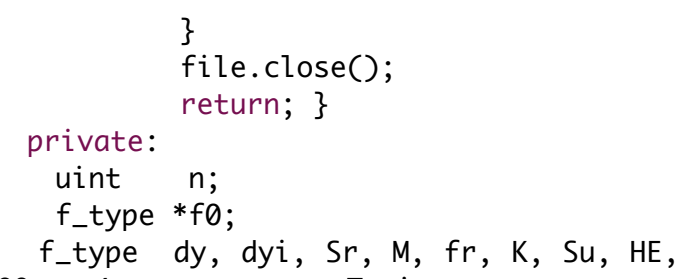

HT, Q0, gm1, tcut, tmax, Tcrit, argt;

\};

int main (int $\operatorname{argc}$, char * const $\operatorname{argv}[]$ ) \{

const uint $\mathrm{n}=20001$;

Stretch $\operatorname{stretch}(n)$;

const f_type $d t=0.001$;

f_type $t=d t * 0.0$;

uint count $=0$;

std: : cout $<$ "Step \tt $($ mu-s $) \backslash t T \backslash t T w "<<$ std: : endl;

f_type $x 52, x 76, x 100$;

while (count<100000) \{

stretch.step $(t, d t)$;

$t+=d t$

++ count;

f_type $\operatorname{Tw} \theta=$ stretch. $\operatorname{Tw}(\theta)$;

if (count\% $400==0$ ) std: : cout $<$ count

$<$ ' $\backslash \mathrm{t}^{\prime}<$ stretch.tau_to_t $(t) * 1 . e 6<<$

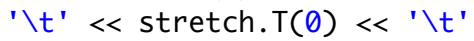

$\ll$ Tw $\theta \ll$ ' $\backslash t^{\prime} \ll$ stretch. $t b(\theta) \ll$

std: : endl;

if (count\%4000==0)

\{ stretch.file_dump(count, 10);

stretch.state_dump(count); \}

if (count $==52000) \times 52=$

stretch. $x \max ()$;

if $($ count $==76000) \times 76=$

stretch. $x \max ()$;

if (count $==100000$ )

$\mathrm{x} 100=$ stretch.$x \max ()$;

\}

$\begin{array}{ll} & \text { std: : cout }<\text { std: : endl ; } \\ / * & \text { std: : cout }<<\end{array}$

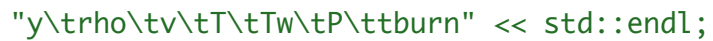

for(uint $i=0 ; i<n ; i+=5$ ) \{

std: : cout $<$ stretch. $y(i)<<' \backslash t '<$

stretch.rho(i) $\ll<' \backslash t$ ' $\ll$ stretch. $v(i)<<$

' $\backslash t^{\prime} \quad<$ stretch.T(i) $<<$

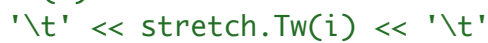

$<$ stretch. $P(i)<<' \backslash t$ ' $<<$

stretch.tau_to_t(stretch.tb(i)) $<<$

std: : endl;

\} $*$ /

// The following calculates the speed of the fluid-phase shock front.

f_type speed $1=(x 76-x 52) /(76.0-52.0)$;

$/ /$ assumes $d t=0.001$

f_type speed2 $=(x 100-x 76) /(100.0-$

76.0); // assumes $d t=0.001$

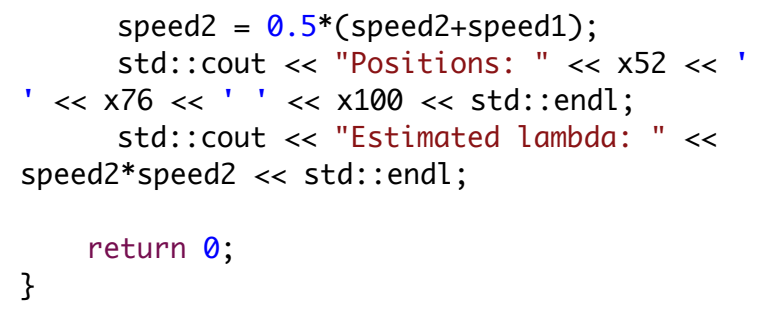




\section{Appendix B. MATLAB Code for RLC Fits to Pulse Waveforms}

\section{RLC fitting routine}

ouse this script to analyize a specific set of pulser data with RLC model

V. Tang, 6/22/09, V1.0

oTakes current waveform data in CSV form and

fits a RLC model to it using

ofminsearch in Matlab. Plots figure for

each fit, and provides summary

ofigures also. Will save all data.

응

-Inputs on top are C, V0, the CVR

attenuation and resistance, and starting

oparameter guesses. Define what you

consider to be to in terms of CVR voltage

and program inputs that into starting guess.

oAlso, input the file numbers you want to analyize in filenumrng

$\%$

ㄴ)-20-09, V2.0

oedits for trigger, self-determined from

Ch4.

ominor edits for plots

\%10-14-10, V3.0

oadded in fitting time time

o07-14-11, V4.0 R. Richardson

ochange to five parameter fit to data;

oFits A, tau, w, to (firing time), and

Baseline current

ㅇequires function RLC Ifit2

odata in TDS3014B csv format; from floppy

07-14-11, V5.0, R. Richardson

omodify to read in CSV file from Openchoice

reader with two channel data;

\% file name mm_dd_yy_pulser_00n.csv

ㅇ8-8-11, V6.0', R. Richardson

omodify to fit to complex roots ; make

compatable with matlab R13 (2002)

RLC_Ifit 3

clear all;

close all;

clear GLOBAL;

global C_global Vo_global

RLC_Ifit_global_curr_out

filenumrng= $[1: 2]$;

OUser Inputs

C_global=8e-9; $\% 8.35 e-9$;

$\mathrm{V} 0 \mathrm{global}=10 \mathrm{e} 3$; $\%$ inital voltage in $\mathrm{V}$

CVR_resist $=0.0140$;

oCVR resist $=0.224$;

CVR_atten=1; $\% 133.5$; $\%$ CVR atten change

$7 / 2 \overline{9} / 11$, was 205 ;

Rguess $=10$;

Lguess $=350 \mathrm{e}-9 ; \% \mathrm{H}$

toguess $=100 e-9 ;$;

alphaguess $=$ Rguess $/ 2 /$ Lguess;

wo $=1 /$ sqrt (Lguess ${ }^{*} \mathrm{C}$ global);

wdguess $=\operatorname{sqrt}($ alphäguess^2-wo^2);

Aguess $=$ - (V0 global/wdguess/Lguess $)$;

params_guess $=[$ Aguess alphaguess wdguess

togues $\bar{s}-.24 * C V R$ atten/CVR_resist ]; oamplitude, decay, frequency, firing time, and scope baseline current

tOVO=-3.5; \%voltage value on CVR trace that defines to initial guess

\%V2 12-20-09 edits

toCV0=1.5; \%voltage for trigger pulse for

trigger time

oV3 10-14-10 optional time after, comment out if don't want

odtfit $=(669-610) * .7$; \% dt for fit in ns

RLC_Ifit_global_curr_out $=0$; \%switch for RLC

fit function

d=dir; oget names of files

shotnumind $=1$;

for $i \mathrm{i}=1$ : length $(\mathrm{d})$

if length(d(ii). name) $>2 \&$

$d(i i)$. name (end -2 : end $)==$ ' $C s v '$

\%if d(ii). name $(20: 22)==$ ' Ch1'

temp1=str 2 num (d (ii). name (16:18));

temp2=find (filenumrng==temp1);

if length (temp2) $==1$

RLC_Ifit_global_curr_out $=0$;

shotnum ( shotnumind)=str 2 num (d (ii). name (16:18 )) ;

ㅇ (ii). name

S1=csvread (d (ii) . name, 17, 3, [17, 3, 9999,4]) ;

och1 data; CVR voltage

S4=csvread (d (ii). name, 17, 9, [17, 9, 9999, 10]) ;

och4 data; camera time

offset $=\operatorname{mean}(\mathrm{S} 1(1: 100,2))$;

\%scope offset

\%dum=find $(\mathrm{S} 1(:, 2)<($ toV0 offset $), 1)$;

$\operatorname{dum}=\min (\mathrm{find}(\operatorname{S1}(:, 2)<($ toV0+offset $)))$; $\%$ for old matlab release

if isempty (dum); dum=1; end :10-14-10 added here to to a dt after shot option

if exist('dtfit','var') \&\&

dtfit $>0$

\%dum2 $=$ find $(\mathrm{S} 1(:, 1)>(\mathrm{S} 1(\mathrm{dum}, 1)+\operatorname{dtfit} \star 1 e-$

9), 1) ;

$\operatorname{dum} 2=\min \left(\right.$ find $\left(\mathrm{S} 1(:, 1)>\left(\mathrm{S} 1(\mathrm{dum}, 1)+\operatorname{dtfit}{ }^{\star} 1 e^{-}\right.\right.$

9)) ) ;

$$
\begin{aligned}
& \mathrm{S} 1=\mathrm{S} 1(1: \operatorname{dum} 2,:) ; \\
& \text { end; } \\
& \text { params_guess }(4)=\mathrm{S} 1(\text { dum, } 1) ;
\end{aligned}
$$

otime in s

params guess (5) $=0$; ㅇfset ${ }^{*} \mathrm{CVR}$ atten/CVR resi st; $\frac{0}{\mathrm{f} f \mathrm{fet}}$ current

$$
\text { t0 }=\operatorname{S} 1(1,1) * 1 \text { e } 9 ;
$$

으 $0=\operatorname{S} 1(1,1) * 1$ e 6 ; $d t=S 1(2,1) * 1$ e $9-t 0$;

$\% d t=S 1(2,1) * 1 e 6-t 0$;

fit in ns

tstart $=200$; ostart time of

in ns 


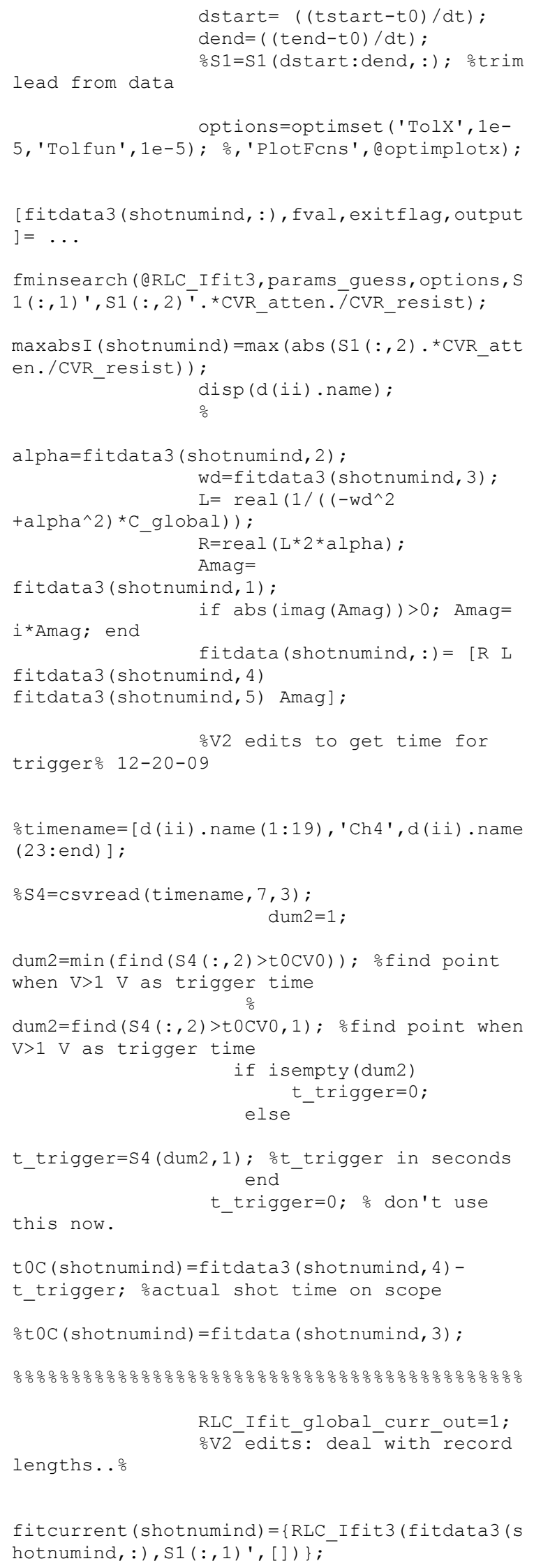


V2 12-20-09 edits for to from trigger\%

Averaget $0 \mathrm{C}=$ mean $(\mathrm{tOC}) ;$ \%average tOC

tocstdev=std (toc);

disp (['Average toc(ns):

', num2str (Averaget0C*1e9), '+/-

', num2str(tocstdev*1e9) ]);

응 Master Plot

$\mathrm{skip}=1$;

save=0;

if $\operatorname{sip}==0$ 900]);

figure; set(gcf,'Position', [100 100600

subplot $(5,1,1)$;

plot ( $\operatorname{shotnum,~fitdata~}(:, 1)$, 'ks') ; ylabel ('R (Oh

m) ') ;

title(['R(ohm), Avg:

', num2str(AverageR, 3), '\pm', num2str (Rstdev, 3

) ] ) ;

hold on;

YAverageR=AverageR*ones (size (fitdata $(:, 1))$ );

plot (shotnum, YAverageR);

ohline (AverageR);

subplot $(5,1,2)$;

plot (shotnum, fitdata $(:, 2) * 1 e 9$, 'bs') ; ylabel ('

$\left.\mathrm{L}(\mathrm{nH})^{\prime}\right)$;

title(['L $(\mathrm{nH})$, Avg:

', num2str (AverageL*1e9,3), '\pm', num2str (Lstd $\mathrm{ev} * 1 e 9,3)]$ );

hold on;

YAverageL=AverageL*1e9*ones (size (fitdata ( : , 1

)) ) ;

plot (shotnum, YAverageL);

ohline (AverageL*1e9);

subplot $(5,1,3)$;

plot (shotnum, maxabsI, 'bs');

ylabel ('Max. Abs. I (A)');

ohline (mean (maxabsI));

hold on;

YmaxI=mean (maxabsI) *ones ( $\operatorname{size}(\operatorname{fitdata}(:, 1)))$

;

plot (shotnum, YmaxI);

title(['Abs. I (A), Avg:

', num2str (mean (maxabsI), 3),' \pm', num2str (std

$(\operatorname{maxabsI}), 3)])$;

\%V2 12-20-09 edits

subplot $(5,1,4)$;

plot (shotnum, toc (:) *1e9, 'gs') ; ylabel ('toc (ns

)');

title(['toc(ns), Avg:

', num2str (Averaget0C*1e9, 3), '\pm', num2str(t0

Cstdev*1e9,3) ]);

hold on;

Yt0C=Averaget0C*1e9*ones $(\operatorname{size}($ fitdata $(:, 1)))$

;

plot (shotnum, Ytoc);

은ine (Averaget0C*1e9);

응

subplot $(5,1,5)$;

plot ( $\operatorname{shotnum,~fitdata~}(:, 3)$ *1e9, 'gs') ; ylabel ('

to (ns) ') ;

title(['to (ns), Avg:

', num2str (Averaget0*1e9,3), '\pm', num2str(t0s tdev*1e9,3) ]);

hold on;

Yt $0=$ Averaget $0 * 1 e 9 *$ ones $(\operatorname{size}($ fitdata $(:, 1)))$;

plot (shotnum, Yt0); \%hline (Averaget0*1e9);

osavetitle=[shotdate ' CVR AnaData, Files

', num2str (shotnum (1)), '-

', num2str (shotnum (end)),'

', datestr (now, 'mm_dd_yy_HH_MM_SS')] ;

savetitle $=[$ shotdate ' $\overline{\text { CVR }}$ Āna $\bar{D}$ ata, Files

', num2str (shotnum (1)),'-

', num2str (shotnum (end)), '

', datestr (now, 30) ] ;

xlabel ( $\{$ 'File

Shot\#'; savetitle\}, 'interpreter', 'none') ;

end

응 save files

if save $==1$

saveas (gcf, savetitle, 'png') ;

saveas (gcf, savetitle, 'fig');

oprint (gcf,'-dtiff', savetitle);

savedataname $=[$ shotdate ' Analyized CVR Data

Fnum ', num2str (shotnum(1)),'-

', num2str (shotnum (end)), '

', datestr (now, 30) ].

osavedataname=[shotdate ' Analyized CVR Data

Fnum ', num2str (shotnum(1)),'-

', num2str (shotnum (end)), '

', datestr (now, 'mm dd yy HH MM SS')] ;

save (savedataname);

end 


\section{Appendix C. MATLAB Code for Fitting Temperature and Density}

\section{Spectral fitting routine Call script}

clear all;

close all;

oscript for 1800 grating (shot xx12 $131516)$

op60s514 1800=csvread ('ooLampCalib 0p2s 25acq_100gain_50slit.asc', 0,0, [ $0 \overline{0}$

2047 1]);

op60s514_1800=csvread ('ooLampCalib_0p2s 25acq_50gain_50slit.asc',0,0,[0 02047

1] ) ;

op60s514 1800=csvread ('ooLampCalib_0p2s 25acq_25gain_50slit.asc',0,0,[0 02047

1] );

op60s514_1800=csvread ('ooLampCalib_0p5s

25acq_20gain_50slit.asc',0,0,[0 02047

1] ) ;

op60s514_1800=csvread ('ooLampCalib_0p5s _25acq_0̄gain_50slit.asc',0,0, [0 $0 \overline{2} 047$

1]);

op60s514_1800=csvread ('ooLampCalib_0p2s

25acq_100gain_50slit.asc', 0, 0, [0 $\overline{0}$

$\overline{2} 0471 \overline{1})$;

\%op60s514_1800=csvread ('ooLampCalib_0p2 s_25acq_150gain_50slit.asc', 0,0, [0 $\overline{0}$

2047 1]);

filestart $=40$

fileend=22;

fileend=filestart;

in.savedata=1; 0 no save, 1 save $\div$

\%op60ns514_1800=csvread ('Lamp_, 0 , 0 , $\left.\left[\begin{array}{llll}0 & 0 & 2047 & \overline{1}\end{array}\right]\right)$;

onew correction from 8/3/2011 cal owavelength_correction $=[1.009592$ $2.67305]$; $\%[1.007578-1.58970]$; wavelength_correction $=[1.0090392$ 2.3592]; ōnew calibration 9_16_11 xnm514=op60s514_1800 (: , 1) *wāvelengt h correction (1) +wavelength correcti on (2);

load calisrc; \%loads LSC1125 CC3

csinterp514=interp1 ( $\operatorname{calisrc}(\overline{:}, 1)$, ca

lisrc $(:, 2), x n m 514$, 'spline');

in.analysis_shot_ind=[filestart: fil eend ]; 응 $[7: 2 \overline{2}]$;

in.eff=op60s514_1800 (:,2) . / csinterp 514 ;

nlines=3; 03 for 3 line fit, 2 for 2 lines; 4 for 4 lines; 1 for special

if $\mathrm{nlines}==4$ in.mainpeaks $=\left[\begin{array}{ll}510.55 & 515.32\end{array}\right.$

$521.82529 .2]$; in.gafactors $=\left[\begin{array}{lll}8 e 6 & 2.4 e 8 & 4.5 e 8\end{array}\right.$

8.72e7] ;

in. $\mathrm{Eu}=\left[\begin{array}{lllll}3.82 & 6.19 & 6.19 & 7.74\end{array}\right]$;

in.man int $\lim =[1.51 .5 ; 2.5$

$2.75 ; 2.75 \overline{2} .5 ; \overline{2} .52 .75]$;

in.man int_lim=[1.1.;1. 1.;1.

$1 . ; 1.51 .4]$;

\%in.man_int_lim=[2 2.;3.3.;3.

$3 ; 12]$

elseif nlines==2 \%first and third

lines.

in.mainpeaks $=[510.55$

521.82 ]

in.gafactors $=\left[\begin{array}{ll}8 e 6 & 4.5 e 8\end{array}\right]$;

in. $\mathrm{Eu}=\left[\begin{array}{ll}3.82 & 6.19\end{array}\right]$;

in.man int $\lim =[1.1 . ; .51]$;

elseif nlines $==3$

in.mainpeaks $=\left[\begin{array}{lll}510.55 & 515.32\end{array}\right.$

$521.82]$;

in.gafactors $=\left[\begin{array}{lll}8 e 6 & 2.4 e 8 & 4.5 e 8\end{array}\right]$;

in. $\mathrm{Eu}=\left[\begin{array}{llll}3.82 & 6.19 & 6.19\end{array}\right]$;

in.man int $\lim =[1.1 . ; 3.3 ; 3$

3.] ; \%[1.0 \overline{1} .0 ; \overline{1} .5 ; 0.81]$;

oin.man_int_lim=[1.5 $1.5 ; 2.5$

3.; 4.53 .5$]$; ㅇat lines

elseif nlines==1 \%first and fouth

line, shot 12 .

in.mainpeaks $=\left[\begin{array}{ll}510.55 & 529.2\end{array}\right]$;

in.gafactors $=\left[\begin{array}{ll}8 e 6 & 8.72 e 7\end{array}\right]$;

in. $\mathrm{Eu}=\left[\begin{array}{ll}3.82 & 7.74\end{array}\right]$;

in.man int $\lim =[1.51 .5 ; .75$ 1];

end;

in. smoothopt =' n' ;

slitw=200; oslit width of

spectrometer

in.blopts $=[2$.09*slitw/50]; $[2$

$.09]$

in. shotid='092111r1';

in.wavelength_correction=wavelength correction;

in.roi range_lim=in.man_int_lim;

in. tholdfactor $=.7$;

in.manual_fit_opt $=1$;

diary ([in.shotid, ' ', datestr (now, 'm mddyy_HHMMSS'), '.txt']);

[a032211, d032211,o032211]=main_call

auto fit V1d2 voigt(in);

if in. savedata $\overline{=}=1$

datatitle=['data_', in. shotid, '_', da

testr (now, 'mmddyy_HHMMSS') ] ; save (datatitle) ;

end;

diary off; 
oclose all;

\section{Voigt profile fitting function}

\section{function}

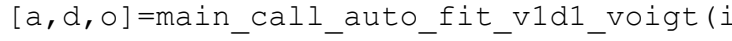
n) ;

\%120909--modified for manual peak input to try to get better result for s3

\section{$\div 020310$}

oVld1--checks for if manual peaks are inputed and then calls auto-fit as oappropiate--i.e. does both regular auto fit call and auto fit 122909 oNow for $\mathrm{Cu}$ also include three points for Star Brodening, based on JPhys. \%Chem Paper 1990, page 1337. (lines $510,515.3,5218.2)$

22310--added linear wavelength correction

\%6-12-10: modified to call voigt profile fits

disp('Main_call_auto_fit_voigt: Version 1.1');

oreadout in structure:

temp=fields (in);

for $i i=1$ :length (temp)

eval ([temp $\left.\{i i\}, '=i n . ', \operatorname{temp}\{i i\},{ }^{\prime} ; '\right]$ );

end;

clear temp;

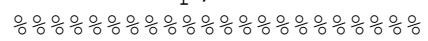

[d] =read_in_asc;

if savedat $\bar{a}==1$

sumplot_title=['All_asc_', shotid, '_', da testr(now, 'mmddyy_H $\bar{H} M M S \bar{S} ')$ ] ; set (gcf, 'PaperPositionMode', 'auto') saveas (gcf, sumplot title, 'fig'); print ('-dpng', ' -

r300', sumplot_title);

end;

$\mathrm{SS}=1 ;$

for $j j=1$ : length (d)

○d $(j j) \cdot \operatorname{spec}(:, 1)=d(j j) \cdot \operatorname{spec}(:, 1)+$ wavele ngth_correction;

$d(j j) \cdot \operatorname{spec}(:, 1)=d(j j) \cdot \operatorname{spec}(:, 1) \cdot{ }^{*}$ wavele ngth_correction (1) +wavelength_correctio n (2);

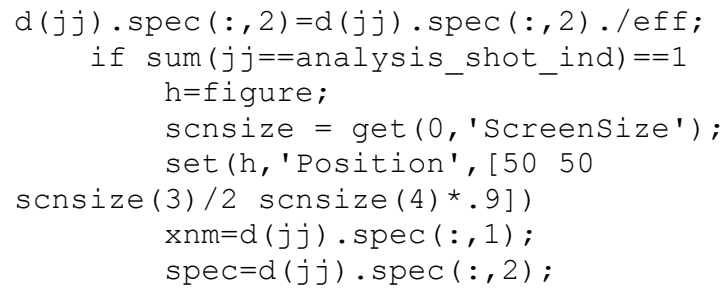

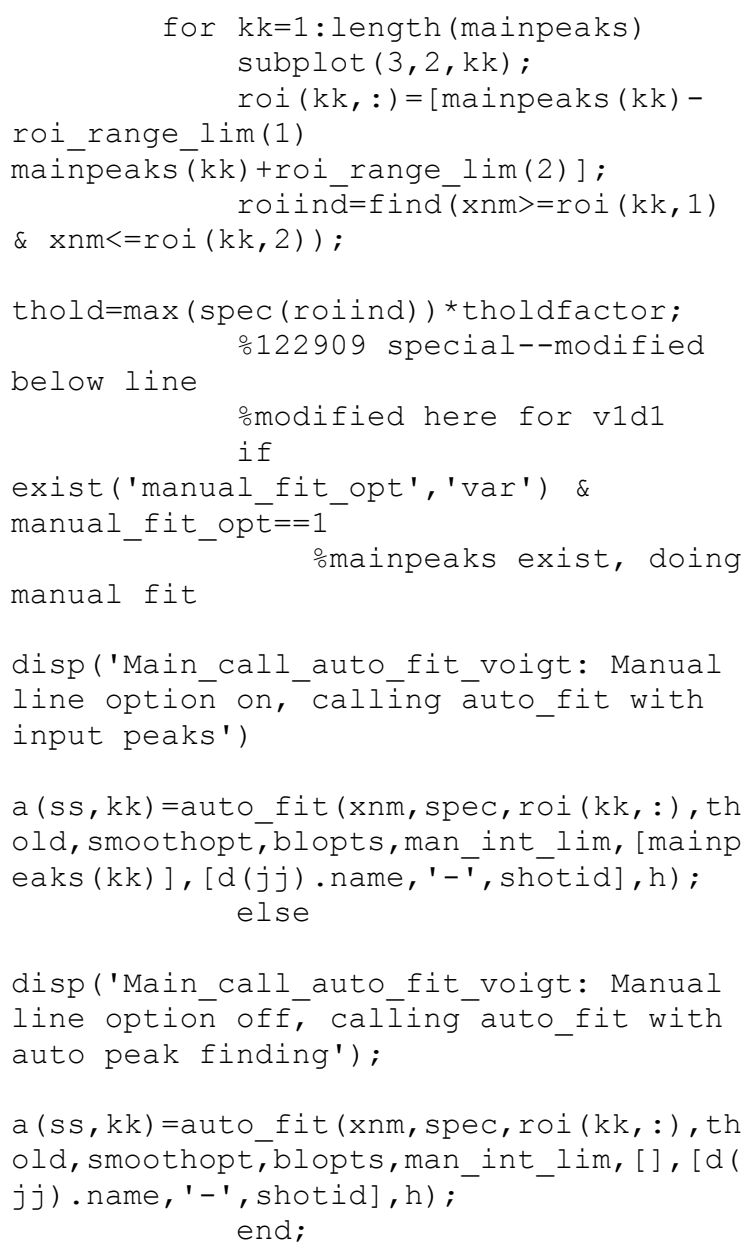




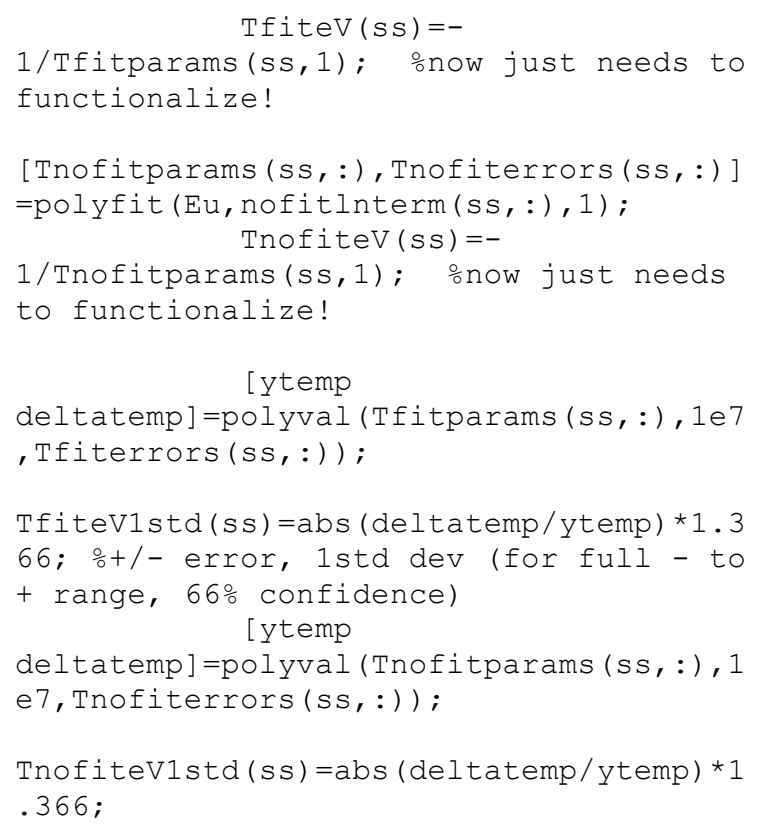

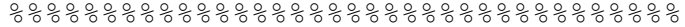

응응 changed 02-03-10 to accomdate new data for stark brodening

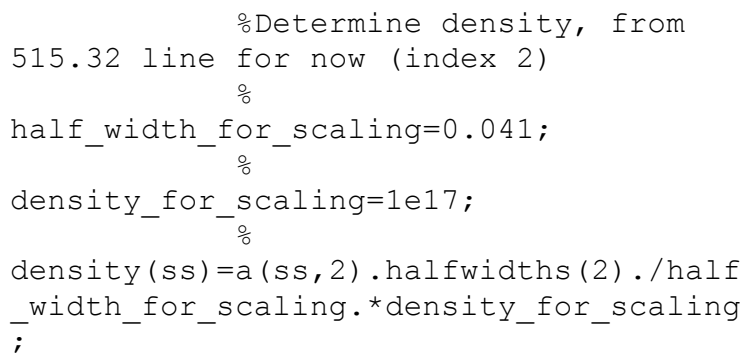


end;

oT summary plots

figure; subplot $(2,1,1)$;

\% size(analysis_shot_ind)

o size (TfiteV)

o size(TfiteV1std)

errorbar(analysis shot ind, TfiteV, Tfite

V1std, TfiteV1std,' 'bs' ' 'Markersize', 6, 'L inewidth', 1.5);

hold on;

errorbar (analysis shot ind, TnofiteV, Tno

fiteV1std, TnofiteV 1 std,' 'rx', 'Markersize

',6, 'Linewidth', 1.5);

xlim([analysis shot ind(1)-1

analysis_shot_ind $(e \bar{n} d)+1])$;

ylabel (' $\bar{T}(\mathrm{eV}) \overline{\mathrm{T}})$;

legend ('T-F', 'T-NF');

subplot $(2,1,2)$;

errorbar(analysis shot ind, density, dens

ity_err, density_err, 'bs' ', 'Markersize', 6

,' Linewidth', 1.5) ;

xlabel(['asc file index--', shotid,' -

:' , datestr (now, 'mmddyy_HHMMSS') ], ' inter

preter', 'none');

legend ('D-515.32');

ylabel ('n(1/cc)');

xlim([analysis shot ind (1) -1

analysis shot $\bar{i}$ ind $(e \bar{n} d)+1])$;

if savedata $=\overline{1}$

figtitle $=[$ ' $\mathrm{T}-$

Den_Sum_', shotid, '_', datestr (now, 'mmddy

Y_H

set (gcf, 'PaperPositionMode', 'auto') saveas (gcf, figtitle, 'fig'); end; print('-dpng',' '-r300',figtitle);

oclear temp variables

clear $h$ jj kk ytemp deltatemp scnsize

spec thold ss

onow read back out.

varnamelist $=$ who:

for $i i=1:$ length (varnamelist)

if strcmp (varnamelist\{ii\},'a') |

strcmp (varnamelist $\{i i\}, ' d ')$ |

strcmp (varnamelist $\{i i\}, '$ in') else

eval (['o.', varnamelist $\{i$ i, ' =' , varnamel

ist $\left\{i\right.$ i,$\left.\left.{ }^{\prime} ; '\right]\right)$; end;

end;

\section{Auto-fit function}

\section{function}

a=auto_fit (x,s,roi, thold, smoothopt, blop ts, man int lim, varargin)

oThis function automically find peaks with an roi for input wavelength $x$ and ospectra s, using threshold thold
oTo indicate peaks manually, input them into varargin by array

$\circ$

○Inputs:

\%: wavelength index

os: spectra/intensity data for $x$

oroi: [x1 $x 2]$ : region of interest in $x$

othold: intensity threshold for peak

finding routine

osmoothopt: 'yx' to smooth data for

peak finding routine--x is number of

opoints to use of smooth; 'n' for no smoothing

oblopts: ==1 for line baseline; ==2 for parabolic

oman int lim: data integration limits-used to compare integrated

ointensities of the data itself with

integrated intensities using the

ofitted Loreztian.--typically [lim1

lim2] nm with lim1=lim2=1 which gives

o+1/-1 nm from peak(if roi allows it--

otherwise goes up to roi limit)

ovarargin: varargin $(1)==[$ peak

wavelengths for peaking] use for manual

oindication of peaks, inistead of using automatic peak finding routine. \%

oOutput:

oa: structure that includes all

variables in function, plus a nice

summary plot

$\circ$

$\%$

oNon-standard Sub_functions required:

(does not require any extra toolboxes):

ocalls peakfinder and lorentz_for_fmin

(included)

ocalls fminsearchbnd

ocalls hline and vline for plots

V. Tang, V1, 12-25/31-09

Ver $=1$; $\frac{\circ}{2}$ version number

plotsyms $=$ '.ox+* $s d v^{\wedge}<>$ ph'; \%setup plot

symbols and colors

plotcolors='bgrcmyk' ;

disp (' ') ;

disp (datestr (now));

disp ('-----------auto_fit------------

$\left.-{ }^{\prime}\right)$

if nargin==7 | isempty (varargin $\{1\}$ ) disp('auto fit: Using automatic

peak finding añd fitting'); else autopeaks=1;

peaks: ')

disp('auto fit: Fitting to inputed

disp (varargin $\{1\})$;

autopeaks=0;

end; 
roiind=find( $x>r o i(1) \quad \& \quad x<\operatorname{roi}(2))$;

sroi=s (roiind);

xroi=x (roiind);

if autopeaks==1

if smoothopt $(1)==$ ' $y$ ' $\%$ smooths out

spectra here for peak founder so we

don't get too many non-peaks--later

fits using these peaks are not smoothed

peaksfoundind=peakfinder (smooth (sroi, st r2num (smoothopt (2))), thold);

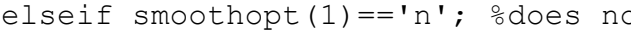
smoothing as requested

peaksfoundind=peakfinder (sroi, thold); end;

elseif autopeaks==0 peaksfoundind=interpl (xroi, $1:$ length (xroi), [varargin $\{1\}]$, 'nearest', 'extrap'); \%find nearest indexes to manual peaks end; peaksfoundx=xroi (peaksfoundind) ; oreturns wavelengths for peaks found using peakfinder in roi region peaksfoundintensity=sroi (peaksfoundind) ; ofound peak intensity for peaks found using peakfiner in roi region

numpeaks=length (peaksfoundx) ; \%number of peaks found

disp (['auto fit: \# of peaks

found/inputēd: ', num2str(numpeaks) ] ) ; disp(['auto fit: Peaks found/inputed in roi: ', num2 str(roi), ', with threshold: ', num2str(thold) ]);

disp ( [peaksfoundx peaksfoundintensity]);

ofitting parameter guess for fit routines

osetup as

$\circ I 1$

$\circ \mathrm{I} 2$

$\div \mathrm{I} 3$

응

\% 02

\%x03, etc.

\%gamma

olast 2 are for baseline if line

olast 3 are for baseline if parabolic

if blopt $s==1$

params guess=[peaksfoundintensity-

mean (sroi); peaksfoundx;

zeros (numpeaks, 1)+.1; $0 ; 0$; ] ; pg $\mathrm{LB}=[$ zeros (numpeaks, 1 );

peaksfoundx-1; zeros (numpeaks, 1); -inf; -inf];

pg $\mathrm{UB}=[$ ones (numpeaks, 1 ) *inf ; peaksfoundx+1; ones (numpeaks, 1) *inf; inf; inf];

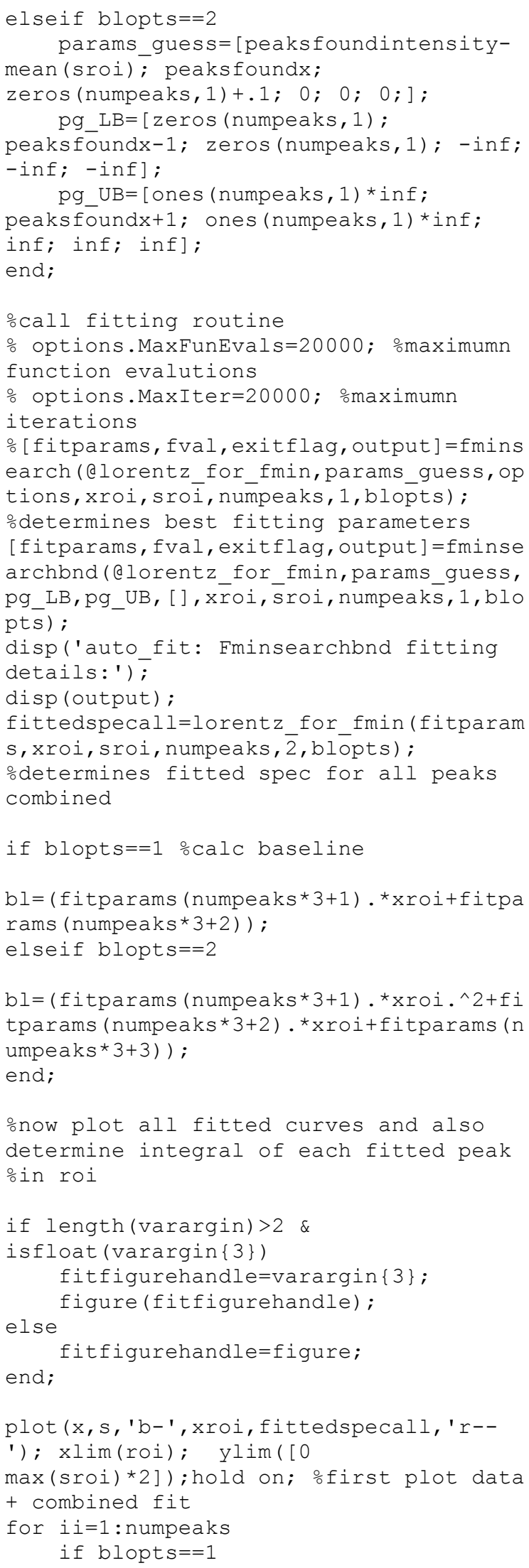




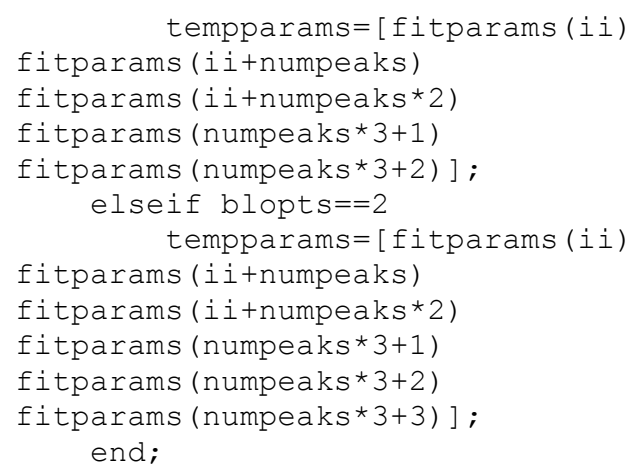

disp(['auto fit: fitted half-width for peak at 
function $y=$ peakfinder (vdata, thold) ofunction $y=$ peakfinder (vdata, thold) oJohn Liptac's peak finding routine \%Version 1.0 Locked 3-16-06

$\div$

Inputs:

ovdata=voltage data with peaks; peaks have to be positive!

othold=threshold votlage

응

ooutput:

oIndex of peaks above threshold

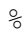

Version 1.0

sat=find (vdata $>=$ thold); $\quad$ subs

above thresh

curve=diff $(\operatorname{sign}(\operatorname{diff}($ vdata $))) ; \quad \frac{\circ}{0}$

look at curvature of peak, $\max =-2$, $\min =2$

lmax=find (curve $==-2)+1 ; \quad \frac{\circ}{0}$

return the subs of local max

imax=intersect (sat, lmax); $\quad$ o take

points with right curvature above

threshold

$\mathrm{y}=\mathrm{imax}$;

function

sse=lorentz for fmin (params, $x$, spectra, $n$

umLor, fitops, blopts)

olet you add up large number of

lorentizians for fitting

$\circ \times 0=10$ cation

$\circ \mathrm{I}=$ height

ogamma=corresponds to width?

oactual output gives errors

응

onumLor=params (1); number of

lorentzians to fit

ostructured like:

I 1

$\div I 2$

$\circ I 3$

$\div \times 01$

$\div \times 02$

\%x03, etc.

olast one is baseline!

for $i i=1$ : numLor end;

○disp (I)

for $i i=1$ :numLor

$$
\text { end; }
$$$$
\text { x0 (ii) =params (ii+numLor); oget x0's }
$$

for $i i=1$ : numLor

gamma (ii)=params $(i i+$ numLor*2) ; oget

gammas's

end;

○disp (gamma)

onow produce lorentizans for input $x$

$\mathrm{y}=0$;

for $i i=1:$ numLor

$\mathrm{y}=\mathrm{I}(\mathrm{i} i) \cdot{ }^{*} \mathrm{gamma}(\mathrm{i} i) \cdot{ }^{\wedge} 2 \cdot /((\mathrm{x}-$

x0 (ii)).^2+gamma (ii) .^2) +y;

end;

onow for baseline

ouses blopts to determine if parabolic baseline is used or not.

if blopts==1 line

blx $1=$ params $($ numLor $* 3+1)$;

blinterp=params (numLor*3+2);

$\mathrm{bl}=\left(\mathrm{bl} \mathrm{xl} \cdot{ }^{*} \mathrm{x}+\mathrm{blinterp}\right)$;

elseif blopts $==2$ oparabolic

bl $1 \times 2=$ params $($ numLor $* 3+1)$;

blx1=params (numLor*3+2);

blinterp=params (numLor*3+3);

$\mathrm{bl}=\left(\mathrm{bl} x 2 \cdot{ }^{\star} \mathrm{x} \cdot{ }^{\wedge} 2+\mathrm{blx} 1 \cdot{ }^{*} \mathrm{x}+\mathrm{b}\right.$ linterp $)$;

end;

$\mathrm{y}=\mathrm{y}+\mathrm{bl}$;

if fitops $==1$

Error Vector=y-spectra; sse=sum (Error_Vector.^2);

odifference in error

elseif fitops==2 oreturn actual vector, with baseline sse $=y$;

elseif fitops $==3$ oreturns intergral,

without baseline sse $=\operatorname{trapz}(\mathrm{x}, \mathrm{y}-\mathrm{bl})$;

end; 
Appendix D. Summer Student Report on Numerical Approach to Hydro Modeling.

\section{Modeling Arc Initiation of Pentaerythritol Tetranitrate: Strategies for Tool Development for High Gradient Hyperbolic Partial Differential Equations}

Han Wang, Vincent Tang, James F. McCarrick, and Christian Grant

Lawrence Livermore National Laboratory

While much research has focused on shock-driven initiation of high explosives (HE) such as pentaerythritol tetranitrate (PETN), few studies have been conducted on prompt thermal initiation from mechanisms such as an electrical arc from electrostatic discharge (ESD). The Weapons Complex Integration (WCI) Arc Initiation Project seeks to understand the reaction mechanisms of arc-initiated HE utilizing computer simulation validated by experimental results. Overall, the simulation model accounts for multispecies convection, interspecies reactions, conduction, radiation cooling, and ohmic heating. MATLAB was chosen as the development tool for its balance of sophistication versus development effort. Optimization was implemented through extensive vectorization and explicit parallelization for multi-core architecture utilizing MATLAB's parallel processing capabilities. Chemical species convections were simulated with a two-dimensional Euler-equation solver based on the MUSCL-Hancock method, and source terms are resolved with the implicit trapezoidal method. This combination yields a second order accurate scheme in space and time. The development phase discovered sensitivity of second order schemes to high gradients, stiff source terms, and grid imprinting. High gradient source terms such as ohmic heating significantly alter the energy landscape in comparison to fluid convection and have to be resolved through a modified use of slope limiters in order to preserve the total variation diminishing (TVD) property of the scheme. In addition, the stiff ordinary differential equations of the chemical reaction rates force the use of multispecies reaction formulas in integral form. Anomalous grid effects, a result of enforcing the Courant-FriedrichsLewy (CFL) condition on Cartesian mesh, were also observed and analyzed in the course of code development. The decomposition of PETN into gas is modeled through a two-step consecutive chemical reaction utilizing Arrhenius reaction rates. Hydrodynamic simulations of the plasma used to drive the chemical reactions match experimental observations within error bars of observed data, demonstrating the utility of the developed hydrodynamic code to explore PETN reactions in the high temperature regime. Future work involving higher order solvers and multipath reaction formulas are required to resolve arc-initiation of PETN at high resolution. 


\section{- I. INTRODUCTION}

Arc initiation of pentaerythritol tetranitrate (PETN) is of paramount importance due to the subjectivity of PETN combustion to spark input energies of ten millijoules [1].

(general background here)

Section II gives the initial conditions and setup used in the simulation. Section III focuses on basic background in the Riemann problem and Euler equation. Section IV details treatment of the inhomogeneous source terms. Section V concentrates on the numerical methods utilized in this program for the homogeneous portion of the partial differential equations solved. Section VI presents numerical results for test cases and real world simulations and section VII entails conclusions.

\section{Simulation Geometry}

In our attempt to study the effects of arc initiation on PETN, we layered PETN film on top of a $\mathrm{CaF}_{2}$ window. The PETN film was surrounded by a layer of isolating Kapton. Parts of the Kapton was layered with $\mathrm{Cu}$ as an electrode.

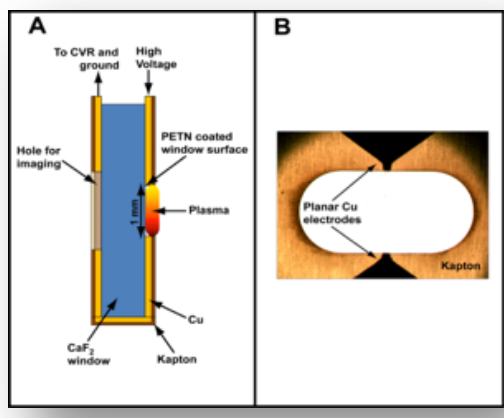

(Figure 1) A. Cutout view of experimental setup, B. View of experimental setup normal to the plane of the electrodes

For the simulation, we modeled the right side of the window in Figure 1A. We ran a two dimensional simulation with the $y$ axis defined as normal to the plane of the window and the $x$ axis defined as coplanar to the window and collinear to the electrodes.

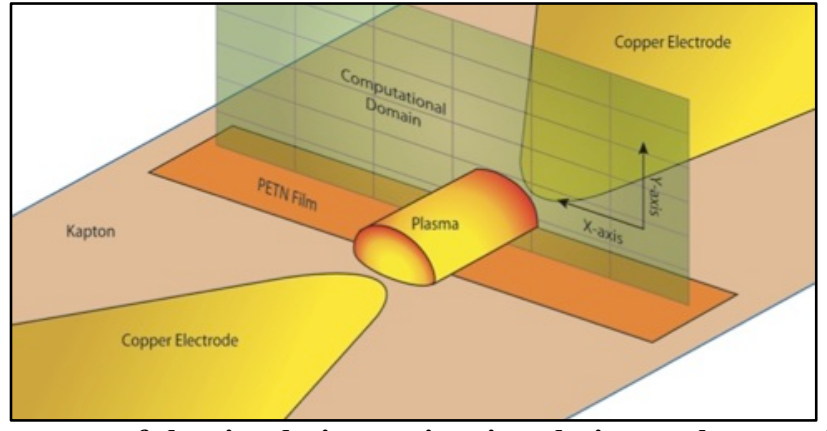

(Figure 2) Placement of the simulation regime in relation to the experimental setup

By our setup, the air and PETN plasma is an expanding half cylinder from the center of the computational domain in $X$ and bottom of the computational domain in $Y$. We discretized the computational domain into $m$ cells in $x$ and $n$ cells in $y$. We utilize a uniform grid and so for a computational domain of $\Delta x_{n e t} \times \Delta y_{n e t}$, each cell is $\frac{\Delta x_{n e t}}{m} \times \frac{\Delta y_{n e t}}{n}$ in size with a net boundary 
length of $2 \frac{\Delta x_{n e t}}{m}+2 \frac{\Delta y_{n e t}}{n}$. For simplicity, we simplify our notation with $\Delta x \equiv \frac{\Delta x_{n e t}}{m}$, and $\Delta y \equiv \frac{\Delta y_{n e t}}{n}$ for the rest of the paper.

In each cell, we track the local density, $\rho$, local momentum in $x, \rho u$, local momentum in $y, \rho v$, and the local energy density, $E$. We define

$$
U \equiv\left[\begin{array}{c}
\rho \\
\rho u \\
\rho v \\
E
\end{array}\right]
$$

with $U_{i, j}$ denoting all the tracked variables in cell $i, j$ st. $1 \leq i \leq m, 1 \leq j \leq n$, and $i, j \in Z$. At the edges of the computational domain, we utilize a layer of ghost cells to simulate reflection and transmission at the boundaries. For a reflective ghost cell, such as those at the bottom of the $y$ axis, we make its orthogonal velocity the negation of its mirror across the computational domain. $U_{R, y}^{\text {ghost }}=\left[\begin{array}{cccc}1 & 0 & 0 & 0 \\ 0 & 1 & 0 & 0 \\ 0 & 0 & -1 & 0 \\ 0 & 0 & 0 & 1\end{array}\right] \times U_{i, j} j \in\{1, n\}$

For a reflecting cell in $x$, we negate the $u$, the $x$ velocity

$U_{R, x}^{\text {ghost }}=\left[\begin{array}{cccc}1 & 0 & 0 & 0 \\ 0 & -1 & 0 & 0 \\ 0 & 0 & 1 & 0 \\ 0 & 0 & 0 & 1\end{array}\right] \times U_{i, j} i \in\{1, m\}$

If we wish to simulate transmission, we make the ghost cell exactly the same as its mirror in the computational domain.

$U_{T, y}^{\text {ghost }}=U_{i, j} j \in\{1, n\}$
$U_{T, x}^{\text {ghost }}=U_{i, j} \quad i \in\{1, m\}$

The depth of the ghost cell layer is decided by the order of the numerical scheme. In our second order scheme, we utilize two layers of ghost cells and for the reflective cells at the bottom of the $y$ axis (Figure 2), they are

$$
\begin{aligned}
& U_{R, y}^{\text {ghost } 1}=\left[\begin{array}{cccc}
1 & 0 & 0 & 0 \\
0 & 1 & 0 & 0 \\
0 & 0 & -1 & 0 \\
0 & 0 & 0 & 1
\end{array}\right] \times U_{i, n} \\
& U_{R, y}^{\text {ghost 2 }}=\left[\begin{array}{cccc}
1 & 0 & 0 & 0 \\
0 & 1 & 0 & 0 \\
0 & 0 & -1 & 0 \\
0 & 0 & 0 & 1
\end{array}\right] \times U_{i, n-1}
\end{aligned}
$$

st. ghost $n$ designates the $n^{\text {th }}$ ghost cell layer. For all ghost cells, transmissive or reflective, their values of $T, p, \rho, E, a$ and $\gamma$ are identical to their mirror counterparts.

\section{Background and Equations}

The time-dependent Euler equations are a system of non-linear hyperbolic conservation laws which govern the behavior of compressible fluids such as gases. In standard literature, the two-dimensional Euler conservation laws are written in vector form as follows

$$
U_{t}+F(U)_{x}+G(U)_{y}=0,(1)
$$




$$
U=\left[\begin{array}{c}
\rho \\
\rho u \\
\rho v \\
E
\end{array}\right], F=\left[\begin{array}{c}
\rho u \\
\rho u^{2}+p \\
\rho u v \\
u(E+p)
\end{array}\right], G=\left[\begin{array}{c}
\rho v \\
\rho u v \\
\rho v^{2}+p \\
v(E+p)
\end{array}\right]
$$

Here, $\rho$ is the mass density, $u$ is the velocity in the $x$ direction, $v$ is the velocity in the $y$ direction, $p$ is the pressure, and $E$ is total energy per unit volume. Also, $U_{t}$ means the partial derivative of $U$ with respect to $t$.

With the addition of $n$ equations, the densities of $n$ chemical species can also be tracked. The modified Euler equations become

$$
\begin{gathered}
U_{t}+F(U)_{x}+G(U)_{y}=0,(3) \\
U=\left[\begin{array}{c}
\rho \\
\rho u \\
\rho v \\
E \\
\rho_{1} \\
\vdots \\
\rho_{n}
\end{array}\right], F=\left[\begin{array}{c}
\rho u \\
\rho u^{2}+p \\
\rho u v \\
u(E+p) \\
\rho_{1} u \\
\vdots \\
\rho_{n} u
\end{array}\right], G=\left[\begin{array}{c}
\rho v v \\
\rho v^{2}+p \\
v(E+p) \\
\rho_{1} v \\
\vdots \\
\rho_{n} v
\end{array}\right] \text { (4) }
\end{gathered}
$$

with $\rho_{n}$ being the density of chemical species $n$. We assume the specie densities to sum to the mass density

$$
\sum_{i} \rho_{i}=\rho .
$$

In the presence of mass, momentum, and energy sinks or sources, the homogenous Euler equations are modified with source terms on the right hand side to model effects other than fluid convection. For arc initiation, we add source terms of conduction, radiation cooling, ohmic heating and chemical reaction.

$$
S(U)=\left[\begin{array}{c}
U_{t}+F(U)_{x}+G(U)_{y}=S(U)(5) \\
0 \\
0 \\
0 \\
\frac{\partial E_{\text {conduction }}}{\partial t}+\frac{\partial E_{\text {radiation }}}{\partial t}+\frac{\partial E_{\text {ohmic }}}{\partial t}+\frac{\partial E_{\text {chem }}}{\partial t} \\
\frac{\partial \rho_{1}}{\partial t} \\
\vdots \\
\frac{\partial \rho_{n}}{\partial t}
\end{array}\right]
$$

Since formulas for each source term may vary depending on the physical systems modeled, they are omitted here. However, formulas used for each source term in the arc simulation are given in section IV, with its numerical treatment in section $\mathrm{V}$. These source term differential equations are solved in conjunction with the convection partial differential equations to model the multiphysics evolution of the system through time.

To close the system of equations used for the simulation, an equation of state is needed. For a good balance between accuracy and computational efficiency, we chose the ideal gas equation of state with weighted $\gamma$ and $R_{\text {specific }}$. Weighing of $\gamma$ and $R_{\text {specific }}$ are done on a particle basis

$$
\gamma=\frac{\sum_{i=1}^{n} \frac{\rho_{i}}{M_{i}} \gamma_{i}}{\sum_{i=1}^{n} \frac{\rho_{i}}{M_{i}}}
$$




$$
R_{\text {specific }}=\frac{\sum_{i=1}^{n} \rho_{i} R_{i}}{\rho},
$$

Where $\gamma_{i}, M_{i}$ and $R_{i}$ are the ratio of specific heats, molar weight, and specific gas constant for specie $i$. More information on our specific EOS can be found in the appendix.

\section{Source Terms}

Homogenous PDDE's describe only the most idealized physical systems and for most applications, source terms are required in the creation of useful models. For arc initiation of PETN, our choice of the guiding equations of conduction, radiation, and ohmic heating are

st. $\mathrm{k}$ is the conduction coefficient,

$$
\frac{\partial E_{\text {conduction }}}{\partial t}=k \cdot \nabla^{2} T \text {, }
$$

$$
\frac{\partial E_{\text {radiation }}}{\partial t}=\frac{8 \pi^{5} k_{b}^{4}}{15 c^{2} h^{3}} k_{g a s} T^{4}
$$

st. $\mathrm{c}$ is the speed of light, $\mathrm{h}$ is Plank's constant, $\mathrm{k}_{\mathrm{b}}$ is the Boltzmann constant, and $\mathrm{k}_{\mathrm{gas}} \mathrm{a}$ normalization factor which accounts for the opacity of the plasma

$$
\frac{\partial E_{o h m i c}}{\partial t}=\frac{J^{2}}{\sigma},
$$

st. $\mathrm{j}$ is the current density and sigma is the plasma conductivity.

Also presented in this section is $\frac{\partial E_{c h e m}}{\partial t}$, the energy change due to chemical reactions, and $\frac{\partial \rho_{i}}{\partial t}$, the change in specie density due to reactions. For brevity, the formulas of $\frac{\partial E_{c h e m}}{\partial t}$ and $\frac{\partial \rho_{i}}{\partial t}$ are omitted until their respective discussions.

These source equations guide the non-convection aspect of our computation domain. To utilize them in our numerical simulations, we need to adapt them to a discrete meshed domain. First, we define our computational domain of $n$ by $m$ cell ( $n$ in the $x$-direction, $m$ in the $y$ direction). $U_{i, j}$ designates the $i^{\text {th }}$ cell in $x$ and the $j^{\text {th }}$ cell in $y$ (Figure 2).

The discretization of the radiation source term is straightforward. We use $k_{g a s}$ as a normalization constraint used to factor in reabsorption of radiation. Reabsorption in the gas depends on the mean free photon path in the plasma which itself depends on the density of the plasma. Here, we chose $k_{g a s}$ is so that $\left(k_{g a s} \rho_{0}\right)^{-1}=1 \mathrm{~cm}$ as in [5]. Essentially, $k_{g a s}$ is chosen so that at normal atmosphere density, $\rho_{0}$, photons will travel a mean free path of $1 \mathrm{~cm}$. Since local cell density will fluctuate, we use the equation

$$
\left(\frac{\partial E_{\text {radiation }}}{\partial t}\right)_{i, j}=\frac{8 \pi^{5} k_{b}^{4}}{15 c^{2} h^{3}} k_{g a s} T_{i, j}^{4} \cdot \rho_{i, j}
$$

to calculate the power loss due to radiation effects in local cell, $U_{i, j}$.

For conduction, we require an estimate of the Laplacian of temperature in every cell. Since we have discrete points of temperature stored at known intervals, we utilize a numerical derivative approximation. We choose a second derivative stencil which is at least second order accurate to maintain the second order spatial accuracy of the entire simulation. A second order centered stencil is

$$
\frac{\partial^{2} T_{i, j}}{\partial x^{2}}=\frac{1}{\Delta x^{2}}\left(T_{i-1, j}-2 T_{i, j}+T_{i+1, j}\right)+O\left(\Delta x^{2}\right)
$$

s.t. $\Delta x$ is the $\mathrm{L} 1$ distance between cell centers in $\mathrm{x}$. In $\mathrm{y}$, the formula becomes

$$
\frac{\partial^{2} T_{i, j}}{\partial y^{2}}=\frac{1}{\Delta y^{2}}\left(T_{i, j-1}-2 T_{i, j}+T_{i, j+1}\right)+O\left(\Delta y^{2}\right)
$$

Substituting both into the Laplacian and plugging back into (7) we obtain 


$$
\left(\frac{\partial E_{\text {conduction }}}{\partial t}\right)_{i, j}=\mathrm{k} \cdot\left(\frac{1}{\Delta x^{2}}\left(T_{i-1, j}-2 T_{i, j}+T_{i+1, j}\right)+\frac{1}{\Delta y^{2}}\left(T_{i, j-1}-2 T_{i, j}+T_{i, j+1}\right)\right)
$$

For cell at the edge of the computational domain, we utilize the fact that the ghost cell's temperature is the same as their neighboring cell to reduce the second derivatives (Section II). For $U_{1,1}$ the stencil becomes

$$
\begin{aligned}
\frac{\partial^{2} T_{i, j}}{\partial x^{2}} & =\frac{1}{\Delta x^{2}}\left(-T_{i, j}+T_{i+1, j}\right)+O\left(\Delta x^{2}\right) \\
\frac{\partial^{2} T_{i, j}}{\partial y^{2}} & =\frac{1}{\Delta y^{2}}\left(-T_{i, j}+T_{i, j+1}\right)+O\left(\Delta y^{2}\right)
\end{aligned}
$$

This result can be easily generalized to other corner cells and boundary cells.

For ohmic heating, we assume the user has data of net current through plasma as a function of time. We note that (9) requires the conductance of the plasma. One method of approximating the conductance of air based on its ionization states is

$$
\begin{gathered}
\sigma(T, \rho)=\frac{4.173 \times 10^{-10}\left(A_{1}+A_{2}\right) T^{-\frac{1}{2}}}{2 \times 10^{-15}\left(1-A_{1}\right)+A_{1} a} \\
A_{1}=\frac{2}{1+\sqrt{2 B_{1}+1}}, \\
A=\frac{2}{1+2 B_{2}+\sqrt{1+6 B_{2}+B_{2}^{2}}}, \\
B_{1}=\rho T^{-1.5} C_{1} \exp \left(\frac{I_{1}}{2 R T}\right), \\
B_{2}=\rho T^{-1.5} \exp ^{\left(\frac{I_{2}}{2 R T}\right)},
\end{gathered}
$$

here, $I_{1}$ and $I_{2}$ are the first and second ionization energies of air. While the approximation of the conductance of air is borrowed from a more sophisticated EOS [5], we have hybridized it with our multispecies ideal gas EOS. We recognize the sub optimality of our hybrid approach but since the ionization EOS requires a root finding procedure in its temperature solution, computational tractability necessitates our hybrid approach. To find an approximation of the scalar field of $\sigma$, we compute $\sigma_{i, j}$ for each cell. We now derive $\frac{\partial E_{o h m i c}}{\partial t}$ as a function of current, I, and $\sigma$. We start with

$$
\frac{\partial E(x, y, t)}{\partial t}=\frac{J^{2}(x, y, t)}{\sigma(x, y, t)}
$$

and utilize the identity $J=\rho \theta$, st. $\theta$ is the electric field strength, for a substitution. We now have

$$
\frac{\partial E(x, y, t)}{\partial t}=\sigma(x, y, t) \theta^{2}(t)
$$

if we assume uniform Electrical field strength in space and by definition, $\theta(t)=\frac{V(t)}{l}=\frac{I(t) R(t)}{l}$ st. $l$ is the distance between the electrodes.

Substituting back into power, we recover

$$
\frac{\partial E(x, y, t)}{\partial t}=\sigma(x, y, t) \frac{I^{2}(t) R^{2}(t)}{l^{2}}(18)
$$

$R(t)$ is the resistance of the entire plasma at time $t$. So to find $R(t)$ we first assume each cell represents a connection from one electrode to the other with conductivity defined by $\sigma_{i, j}$. Each cell now represents a conducting column of plasma connecting the electrodes in parallel. We first find the resistance of each cell,

$$
R_{i, j}=\rho \cdot \frac{l}{A}=\frac{l}{\sigma_{i, j} \Delta x \Delta y}
$$

Since the columns of plasma are essentially resistors in parallel, the net resistance is 


$$
\begin{gathered}
\frac{1}{R_{n e t}}=\sum_{i} \sum_{j} \frac{1}{R_{i, j}}=\sum_{i} \sum_{j} \frac{\sigma_{i, j} \Delta x \Delta y}{l}, \\
R=l\left(\sum_{i} \sum_{j} \frac{1}{\sigma_{i, j} \Delta x \Delta y}\right)(20) .
\end{gathered}
$$

Substitute (20) into (18) and we recover the desired form of the ohmic heating equation [4]

$$
\left(\frac{\partial E_{\text {ohmic }}}{\partial t}\right)_{i, j}^{t}=\frac{I^{2}(t)}{\left(\sum_{i} \Sigma_{j} \sigma\left(T_{i, j}^{t}, \rho_{i, j}^{t}\right) \Delta x \Delta y\right)^{2}} \sigma\left(T_{i, j}^{t}, \rho_{i, j}^{t}\right)
$$

This equation defines ohmic heating of cell $U_{i, j}$.

For the chemistry equations, we started with the differential equations of a consecutive reaction $[A] \rightarrow[B] \rightarrow[C]$. This set of reaction equations is based on the simplified assumption of PETN undergoing complete ionization before recombining to form products.

$$
\begin{gathered}
\frac{d[A]}{d t}=-k_{1}[A]_{0}, \\
\frac{d[B]}{d t}=k_{1}[A]_{0}-k_{2}[B]_{0}, \\
\frac{d[C]}{d t}=k_{2}[C]_{0} .
\end{gathered}
$$

The discussion about the choice for an Arrhenius model of the rate constant, $k$, will be detailed in section IV and will not be repeated here. Now, we propose the solution of the above differential equations in integral form after a time $t$ has elapsed. They are

$$
\begin{gathered}
{[A]=[A]_{0} e^{-k_{1} t},(22)} \\
{[B]=[A]_{0} \frac{k_{1}}{k_{2}-k_{1}}\left(e^{-k_{1} t}-e^{-k_{2} t}\right)+[B]_{0} e^{-k_{2} t},} \\
{[C]=[A]_{0}\left(1+\frac{k_{1} e^{-k_{2} t}-k_{2} e^{-k_{1} t}}{k_{2}-k_{1}}\right)+[B]_{0}\left(1-e^{-k_{2} t}\right)+[C]_{0} .}
\end{gathered}
$$

Inputting the initial chemical concentrations at the start of the source time step, we chose $t=\frac{\Delta t}{2}$ (the choice of $\frac{\Delta t}{2}$ is due to source splitting) and recover a closed form solution of the chemical system at the end of each source step. We define $[A]_{\frac{\Delta t}{2}},[B]_{\frac{\Delta t}{2}}$, and $[C]_{\frac{\Delta t}{2}}$ to designate the chemical concentrations at the end of the source time step. We also define the heat of formation as $Q_{A}, Q_{B}$, and $Q_{C}$ for chemical specie $A, B$, and $C$, respectively. Then the integral average of $\frac{\partial E_{\text {chem }}}{\partial t}$, $\frac{\partial \rho_{1}}{\partial t}, \frac{\partial \rho_{2}}{\partial t}$, and $\frac{\partial \rho_{3}}{\partial t}$ during the source time step becomes

$$
\begin{aligned}
& \left(\frac{\overline{\partial E_{\text {chem }}}}{\partial t}\right)_{i, j}^{0}=\frac{2}{\Delta t}\left[Q_{A}\left([A]_{i, j}^{\frac{\Delta t}{2}}-[A]_{i, j}^{0}\right)-Q_{B}\left([B]_{i, j}^{\frac{\Delta t}{2}}-[B]_{i, j}^{0}\right)-Q_{C}\left([C]_{i, j}^{\frac{\Delta t}{2}}-[C]_{i, j}^{0}\right)\right], \\
& \left(\frac{\overline{\partial \rho_{1}}}{\partial t}\right)_{i, j}^{0}=\frac{2}{\Delta t}\left([A]_{i, j}^{\frac{\Delta t}{2}}-[A]_{i, j}^{0}\right) \text {, } \\
& \left(\frac{\overline{\partial \rho_{2}}}{\partial t}\right)_{i, j}^{0}=\frac{2}{\Delta t}\left([B]_{i, j}^{\frac{\Delta t}{2}}-[B]_{i, j}^{0}\right) \text {, } \\
& \left(\frac{\overline{\partial \rho_{3}}}{\partial t}\right)_{i, j}^{0}=\frac{2}{\Delta t}\left([C]_{i, j}^{\frac{\Delta t}{2}}-[C]_{i, j}^{0}\right) \text {. }
\end{aligned}
$$

Now that we have discrete derivative formulas for each source term, we can combine them into a time stepping scheme that goes from the initial condition to $\frac{\Delta t}{2}$. We reformulate the implicit trapezoidal method with chemical source terms as 


$$
\begin{aligned}
& E_{i, j}^{\frac{\Delta t}{2}}= \\
& E_{i, j}^{0}+\frac{\Delta t}{2}\left(\frac { 1 } { 2 } \left[\left(\frac{\partial E_{\text {ohmic }}}{\partial t}\right)_{i, j}^{0}+\left(\frac{\partial E_{\text {conduction }}}{\partial t}\right)_{i, j}^{0}+\left(\frac{\partial E_{\text {radiation }}}{\partial t}\right)_{i, j}^{0}+\left(\frac{\partial E_{\text {ohmic }}}{\partial t}\right)_{i, j}^{\frac{\Delta t}{2}}+\left(\frac{\partial E_{\text {conduction }}}{\partial t}\right)_{i, j}^{\frac{\Delta t}{2}}+\right.\right. \\
& \left.\left(\frac{\partial E_{\text {radiation }}}{\partial t}\right)_{i, j}^{\frac{\Delta t}{2}}\right]+\left(\frac{\left.\overline{\frac{\partial E_{\text {chem }}}{\partial t}}\right)_{i, j}^{0}}{\Delta,},\right. \\
& \rho_{1, j}^{\frac{\Delta t}{2}}=\rho_{1, j}^{0}+\frac{\Delta t}{2} \frac{\overline{\partial \rho_{1}}}{\partial t}=[A]_{i, j}^{\frac{\Delta t}{2}}, \\
& \rho_{2}{ }_{i, j}^{\frac{\Delta t}{2}}=\rho_{2 i, j}^{0}+\frac{\Delta t}{2} \frac{\overline{\partial \rho_{2}}}{\partial t}=[B]_{i, j}^{\frac{\Delta t}{2}}, \\
& \rho_{3, j}^{\frac{\Delta t}{2}}=\rho_{3 i, j}^{0}+\frac{\Delta t}{2} \frac{\overline{\partial \rho_{3}}}{\partial t}=[C]_{i, j}^{\frac{\Delta t}{2}} \text {. }
\end{aligned}
$$

A flowchart for our algorithm detailing our implementation of the predictor-corrector method with explicit chemical terms is found in the appendix.

\section{Numerical Methods}

In our simulations, we chose numerical schemes based on the finite volume method. Many published literature have shown the superior resolution of finite volume methods for problems involving high gradient shock wave [3]. The finite volume method divides up the computational regime into discrete computational cells. The cell averaged values of the conservative variables are stored in the geometrical center. The inter-cell interactions are then solved through computations of the Riemann problem.

A Riemann problem for the Euler equations is a set of initial conditions which satisfy the follow the requirements

$$
U(x, 0)=\left\{\begin{array}{ll}
U_{L} & x<0 \\
U_{R} & x>0
\end{array}(32)\right.
$$

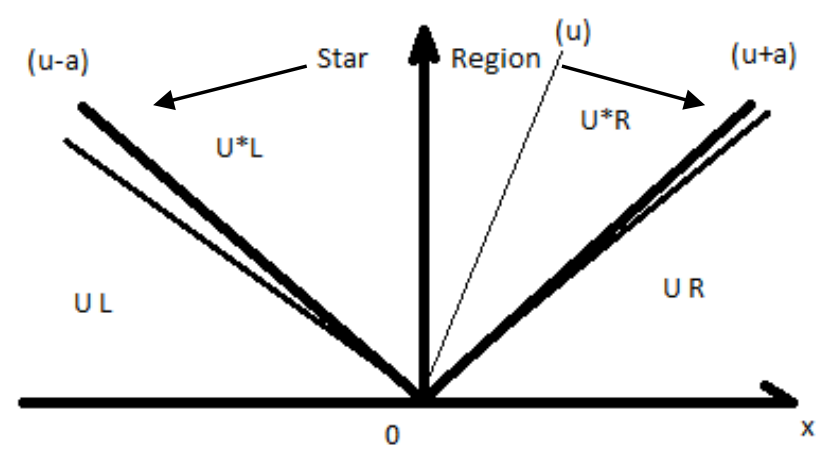

(Figure 3) General solution of the linearized Riemann problem

The Riemann problem yields wave fronts propagating from the inter-cell contact surface. Of the three waves, the outside waves with velocities $(u-a)$, and $(u+a)$ are either rarefaction or shock waves; the center wave is always a contact wave. More information on Riemann wave fronts can be found in [3]. The solution of the Riemann problem also yields the value of the conservative variables in the interwave regions. Since the definition of a cell's conservative variables comes from the integral average through the cell, the wave speeds and interwave values given by the Riemann problem solution allows for an easy and intuitive way to advance the computational domain through time. After the Riemann solution, the wave fronts are advanced $\Delta t$ in time and the cell average recalculated. Courant showed that as long as $\Delta t$ is taken small 
enough that the leading wave fronts of left and right cells do not interfere, each cell's progression in time can be calculated through its boundary interactions with its neighboring cells, this is result is call the Courant-Friedrichs-Lewy condition [3].

$$
\Delta t \leq \frac{\Delta x}{u_{\max }}(33)
$$

Here $u_{\max }$ means the fastest possible wave speed resulting from the solution to the Riemann Problem. Once the solution of the intermediate regions $U_{i+\frac{1}{2}}$ and $U_{i-\frac{1}{2}}$ is known, the cell average can be advanced through time by the Godunov method

$$
U_{i}^{n+1}=U_{i}^{n}+\frac{\Delta t}{\Delta x}\left[F\left(U_{i-\frac{1}{2}}^{n}\right)-F\left(U_{i+\frac{1}{2}}^{n}\right)\right]
$$

The significance of all Riemann solvers is that they incorporate upwind information into the approximation of the inter-cell region $U_{i+\frac{1}{2}}$ which is more accurate than the simple numerical average of

$$
U_{i+\frac{1}{2}}=\frac{1}{2}\left[U_{i}+U_{i+1}\right]
$$

However, an exact solution has to be obtained iteratively making the solution very computationally costly. Thus for a solution to the Riemann problem, we forgo the exact Riemann solver for sake of computational efficiency as is typically done [3] and chose the Harten, Lax, van-Leer, and Contact (HLLC) solver. Out of the main Riemann solvers in literature, the Roe Riemann solver and the HLLC solver are the only ones which resolve the intermediate contact wave between cells. However, the HLLC solver is about $40 \%$ more computational efficient [6] and because we intend to deploy our code on a desktop computer, we chose the HLLC solver for the additional computational efficiency.

The intercell region for the HLLC solver is defined as

$$
\begin{gathered}
U_{i+\frac{1}{2}}=\left\{\begin{array}{lc}
U_{L}, & 0 \leq S_{L} \\
U_{* L}, & S_{L} \leq 0 \leq S_{*} \\
U_{* R}, & S_{*} \leq \leq S_{R} \\
U_{R}, & S_{R} \leq 0
\end{array}\right\} \\
U_{* K}=\left(\frac{S_{K}-u_{k}}{S_{k}-S_{*}}\right)\left[\begin{array}{c}
S_{*} \\
v_{K} \\
\frac{E_{K}}{\rho_{K}}+\left(S_{*}-u_{K}\right)\left[S_{*}+\frac{p_{K}}{\rho_{K}\left(S_{K}-u_{K}\right)}\right] \\
\rho_{1} \\
\vdots \\
\rho_{n}
\end{array}\right]
\end{gathered}
$$

with $S_{L}$ and $S_{R}$ being estimates for the wavespeeds of the rightmost and leftmost waves. Instead of utilizing a simple direct estimate for $S_{L}$ and $S_{R}$ [5], we estimated for the wavespeeds in our program through pressure. Let $K \in\{L, R\}$ and our estimate for $S_{K}$ becomes

Where

$$
S_{K}=u_{K}-a_{K} q_{K},(38)
$$

$$
q_{K}=\left\{\begin{array}{cc}
1 & p_{*} \leq p_{K} \\
{\left[1+\frac{\gamma+1}{2 \gamma}\left(\frac{p_{*}}{p_{k}}-1\right)\right]^{\frac{1}{2}}} & p_{*}>p_{k} .
\end{array}\right.
$$


Notice, we now require an estimate for $p_{*}$ (pressure estimate of the intermediate wave region), and we obtain this estimate through the Adaptive Noniterative Riemann Solver (ANRS). This is a primitive variable Riemann Solver which is even more computationally cheap than the HLLC. For brevity, details of the ANRS can be found in [3].

After obtaining $S_{L}$ and $S_{R}$, we can now compute $S_{*}$ which is used for the piecewise evaluations in (36).

$$
S_{*}=\frac{p_{R}-p_{L}+\rho_{L} u_{L}\left(S_{L}-u_{L}\right)-\rho_{R} u_{R}\left(S_{R}-u_{R}\right)}{\rho_{L}\left(S_{L}-u_{L}\right)-\rho_{R}\left(S_{R}-u_{R}\right)},(40)
$$

Finally, we now evaluate (36) with the calculated $S_{*}$ for $U_{i+\frac{1}{2}}$. After $U_{i+\frac{1}{2}}$ is found, we define the HLLC intercellular flux as,

$$
F_{i+\frac{1}{2}}=F\left(U_{i+\frac{1}{2}}\right)
$$

and return $F_{i+\frac{1}{2}}$ back to the numerical scheme of choice. In two dimensions, the fluxes are solved via dimensional splitting. Two passes of the HLLC solver are made through the computational domain, the first pass solves for $F_{i \pm \frac{1}{2}, j}$ ( $x$ direction), while the second pass solves for $F_{i, j \pm \frac{1}{2}}(y$ direction). When solving for $U_{*}$ in the $y$ direction, the formula changes to

$$
U_{* K}=\left(\frac{S_{K}-v_{k}}{S_{k}-S_{*}}\right)\left[\begin{array}{c}
\rho \\
v_{K} \\
S_{*} \\
\frac{E_{K}}{\rho_{K}}+\left(S_{*}-v_{K}\right)\left[S_{*}+\frac{p_{K}}{\rho_{K}\left(S_{K}-v_{K}\right)}\right] \\
\rho_{1} \\
\vdots \\
\rho_{n}
\end{array}\right] .
$$

Many options exist for a second order total variation diminishing (TVD) scheme [3] and we chose the MUSCL-Hancock method [3]. As a member of the Monotone Upstream-Centered Scheme for Conservation Laws (MUSCL) class methods, MUSCL-Hancock relies on spatial reconstruction of the data inside each computational cell.

MUSCL-Hancock aims to supply more accurate initial conditions to the Riemann Problem. First, linear data reconstruction is done on the interfaces of each cell to find a left and right interface value, namely

$$
\begin{gathered}
U_{i}^{L}=U_{i}^{n}-\frac{1}{2} \Delta i, \quad U_{i}^{R}=U_{i}^{n}+\frac{1}{2} \Delta i, \\
\Delta i=\frac{U_{i+1}^{n}-U_{i-1}^{n}}{2} .(43)
\end{gathered}
$$

Then the interface values are evolved in time for $\frac{1}{2} \Delta t$ according to

$$
\begin{gathered}
\bar{U}_{i}^{L}=U_{i}^{L}+\frac{\Delta t}{2 \Delta x} F_{\text {time }}, \quad \bar{U}_{i}^{R}=U_{i}^{R}+\frac{\Delta t}{2 \Delta x} F_{\text {time }}, \\
F_{\text {time }}=\left[F\left(U_{i}^{L}\right)-F\left(U_{i}^{R}\right)\right] .
\end{gathered}
$$

Finally, the values $\bar{U}_{i}^{L}$ and $\bar{U}_{i}^{R}$ are reconstructed from $U_{i-1}$ and $U_{i}$ are used as initial conditions of a piece-wise constant Riemann Problem on the left

$$
U(x, 0)= \begin{cases}\bar{U}_{i}^{R}, & x<0 \\ \bar{U}_{i+1}^{L}, & x>0\end{cases}
$$

and the values $\bar{U}_{i}^{L}$ and $\bar{U}_{i}^{R}$ reconstructed from $U_{i}$ and $U_{i+1}$ are used as initial conditions of a piece-wise constant Riemann Problem on the right. 


$$
U(x, 0)=\left\{\begin{array}{ll}
\bar{U}_{i}^{L}, & x<0 \\
\bar{U}_{i+1}^{R}, & x>0
\end{array},\right.
$$

The solutions obtained from the Riemann Problem is then used to update the cell average of $U_{i}$ through

$$
U_{i}^{n+1}=U_{i}^{n}+\frac{\Delta t}{\Delta x}\left[F\left(U_{i-\frac{1}{2}}\right)-F\left(U_{i+\frac{1}{2}}\right)\right] .
$$

The MUSCL-Hancock method is analogous to combining two integration rules, the trapezoidal rule in space and the midpoint rule in time. A detailed flowchart of the MUSCL-Hancock method is included in the appendix.

While the MUSCL-Hancock method allows a $2^{\text {nd }}$ order accurate solution in space and time, it yields spurious oscillations in the neighborhood of high gradients. Literature advocates the use of limited slopes, $\bar{\Delta} i$, to smooth out gradient induced oscillations. Limited slopes are defined as

$$
\bar{\Delta} i=\varphi(r) \cdot \Delta i
$$

st. $r \equiv \frac{U_{i+1}^{n}-U_{i}^{n}}{U_{i}^{n}-U_{i-1}^{n}}$, and $\varphi(r)$ is the limiter function.

One popular choice for a limiter function which we found to be robust and accurate is SUPERBEE [3]

$$
\varphi_{\text {super } B}(r)=\left\{\begin{array}{lr}
0, & r \leq 0 \\
2 r, & 0 \leq r \leq \frac{1}{2} \\
1, & \frac{1}{2} \leq r \leq 1 \\
\min \left\{r, \frac{2}{1+r}, 2\right\}, & r \geq 1
\end{array} .\right.
$$

Nevertheless, even with limited slopes, we still encountered computational failures when high gradient source terms are coupled with the advection PDE's. In our system, the ohmic heating term could increase energy density in certain cells by more than $200 \%$ between convection time steps. We experimented with placing the limiter on $\rho, E, p, \rho u$, and $\rho v$ but no matter what variable, conservative or primitive, we chose for the limiter, all simulations would succumb to spurious oscillations.

Acting on the observation that simulation failures for different choices of variables occurred at different times, we reformulated $r$ as $r_{\text {min. }}$

$$
r_{\text {min }}=\min \left\{\begin{array}{c}
r_{\rho} \\
r_{\rho u} \\
r_{E}
\end{array}\right\}
$$

This simple reformation guarantees the data reconstruction only occurs in regions where all conservative variables are smooth. This protects the scheme from succumbing to source terms which make sharp changes to a single conservative variable. Empirically, the use of $r_{\text {min }}$ has led to stability for energy source terms in our simulations.

Treatment of the right hand of (5) is done via source splitting. First, we define $S^{(t)}$ as the solution operator for the right hand side of (5) and $C^{(t)}$ as the solution operator for the left hand side of (5). Then source splitting defines the updated full time step as

$$
U^{n+1}=S^{\left(\frac{\Delta t}{2}\right)}\left[C^{(\Delta t)}\left[S^{\left(\frac{\Delta t}{2}\right)}\left(U^{n}\right)\right]\right] .
$$

Essentially, the solution to the ODE source problem for $1 / 2$ the time step is taken as the initial condition of the convection problem for a full time step. Then the solution of the convection 
problem is taken as the initial condition for the source problem for another $1 / 2$ time step and the resulting solution taken as the solution for the entire scheme. For our purposes, $C^{(\Delta t)}$ is the MUSCL-Hancock scheme for $\Delta t$ and $S^{(\Delta t)}$ is the implicit trapezoidal method for our chosen source terms (6). Due to our numerical treatment of the source terms (see section III), $\Delta t$ is only restricted by the CFL condition.

We chose implicit trapezoidal method for most source term calculations because the implicit nature of the method allowed us to avoid making multiple EOS calls per cell, per time step. This is because all explicit methods rely on closed forms of derivatives with respect to time. However, all of our energy source terms (radiation, ohmic, conduction, and chemical reaction) are temperature dependent, forcing us to utilize the chain rule in conjunction with the EOS to obtain a $2^{\text {nd }}$ order approximation. While normally the use of the chain rule on the ideal gas EOS does not result in huge overheads, it does in the case of noninvertible EOS's where $\frac{\partial T}{\partial E}$ has to be approximated numerically with a stencil. This result in a minimum of three EOS calls per time step versus the implicit trapezoidal method's two. Also, implicit trapezoidal is unconditionally A-stable, a feature which any explicit method lacks.

For the chemistry source term, we did not implement the implicit trapezoidal rule. This is because the Arrhenius reaction rates,

$$
k=A e^{-\frac{E}{R T}},(53)
$$

where $\mathrm{k}$ is reaction constant, $\mathrm{A}$ is collision frequency, $\mathrm{E}$ is activation energy and $\mathrm{R}$ is universal gas constant, are exponential with respect to temperature.

While the implicit trapezoidal method works fine for the chemical reactions in differential form, regions in the simulation with high temperatures predicted reaction rates consuming over $20 \mathrm{x}$ the available PETN in the cell in a single time step. This would force the use of a time step about $\frac{1}{20}$ the size of the required CFL limited time step. This is highly undesirable because it increases computation run time for little to no gain in resolution.

We instead resort to the integral form of the chemical reactions. However, the accuracy of the integral form of chemical reactions depends on the reaction rate supplied as the close formed expression results from the assumption of a constant reaction constant for the time step. In a $\Delta t$ where the temperature can vary wildly, the integral form will not give a good approximation of the solution. Two solutions to alleviate this problem are take a smaller time step, or find a reaction rate which best represents the chemical system for the entire range of temperatures in the $\Delta t$. Since we have already decided that the first solution is computationally infeasible, we utilize solution two. We model the reaction rate, $k$, through the Arrhenius model and note that $k$ is dependent only on temperature. The ideal temperature supplied to the Arrhenius model would be the integral average temperature of the time step,

$$
T_{\text {int }}=\frac{1}{\Delta t} \int_{0}^{\Delta t} T(x) d x .
$$

However this forces us to obtain the temperature at every intermediate point in the interval $[0, \Delta t]$. We instead settle for the $2^{\text {nd }}$ order approximation of $T_{\text {int }}$. First we use the trapezoidal rule on the integral

$$
T_{\text {int }}=\frac{1}{\Delta t}\left((\Delta t-0)\left(\frac{(T(0)+T(\Delta t)]}{2}\right)+O\left(\Delta t^{2}\right)\right)
$$

Then we move the truncation error to the left hand side and simplify. The result,

$$
\bar{T} \equiv \frac{T(0)+T(\Delta t)}{2}=T_{\text {int }}+O\left(\Delta t^{2}\right)
$$


shows that the simple numerical average can be an acceptable approximation for temperature. Because our overall scheme is only $2^{\text {nd }}$ order accurate, a finer approximation would only result in higher computation time and no gain of accuracy. Thus, we take the average temperature of the cell within the source time step, to supply a more accurate temperature for Arrhenius model.

$$
\bar{T}=\frac{T_{0}+T_{\Delta t}}{2}
$$

The choice for $T_{0}$ as the initial condition temperature is obvious but the choice of $T_{\Delta t}$ lies open. Our method is to take the temperature computed by the corrector step from the other source terms. Notice this choice does not account for the energy released and/or absorbed by the chemical reaction in this time step until the next time step. Mathematically, the decoupling of chemical reaction energy until the next time step can be seen in (25). Nevertheless, this problem is much alleviated by the fact that we take two source steps per convection step. A detail treatment of the use of Arrhenius reaction rates is given in Section III, and a flowchart of our algorithmic implementation is given in the appendix.

\section{Numerical Tests and Results}

Our first test is chosen to document the stability of our numerical solver for a purely convection based problem. For the convection benchmark, we took a cylindrical explosion test problem from [3]. In this test, the initial condition is chosen such that a cylinder of high pressure and volume is placed at the center of the computational domain. Mathematically, the setup is

$$
\begin{aligned}
& \rho=\left\{\begin{array}{ll}
.125 & r>.4 \\
1 & r \leq .4
\end{array},\right. \\
& p=\left\{\begin{array}{ll}
1 & r>.4 \\
1 & r \leq .4
\end{array},\right.
\end{aligned}
$$

with $r$ being the radial distance from the center of the computational domain. We evolved the system for .25 time units and the simulation predicted the following behavior

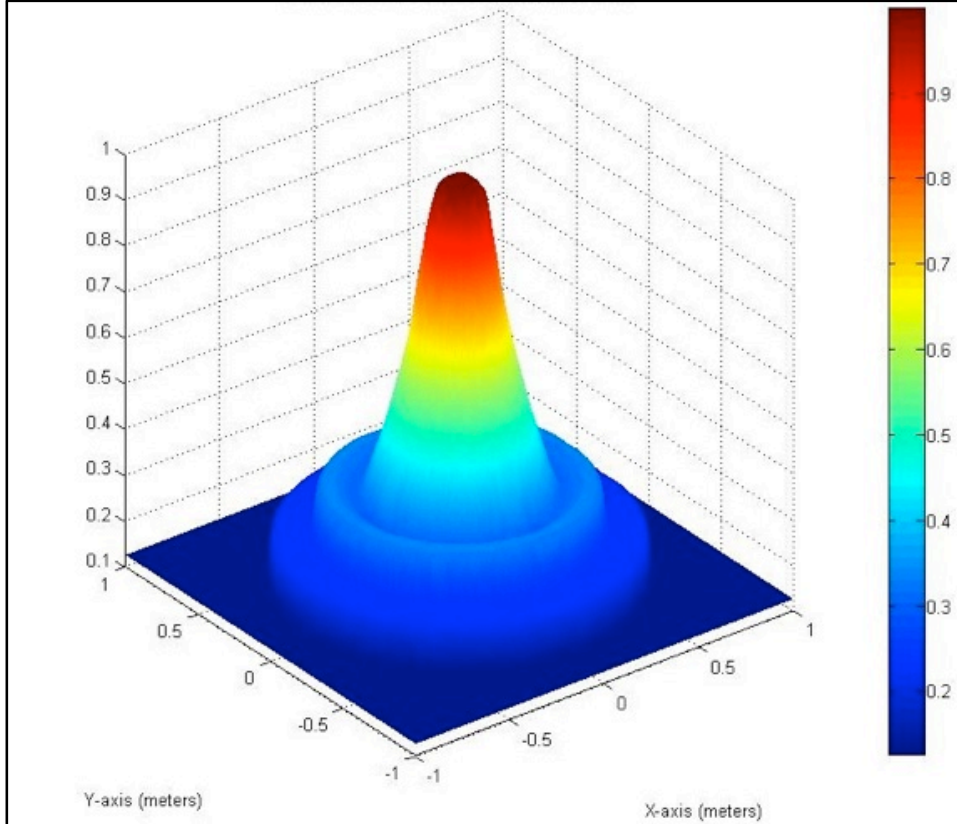

(Figure 4) Convection Test Case after .25 Time Units 
We benchmarked the results with our one dimensional solver which is known stable and obtain the following lineout as a comparison.

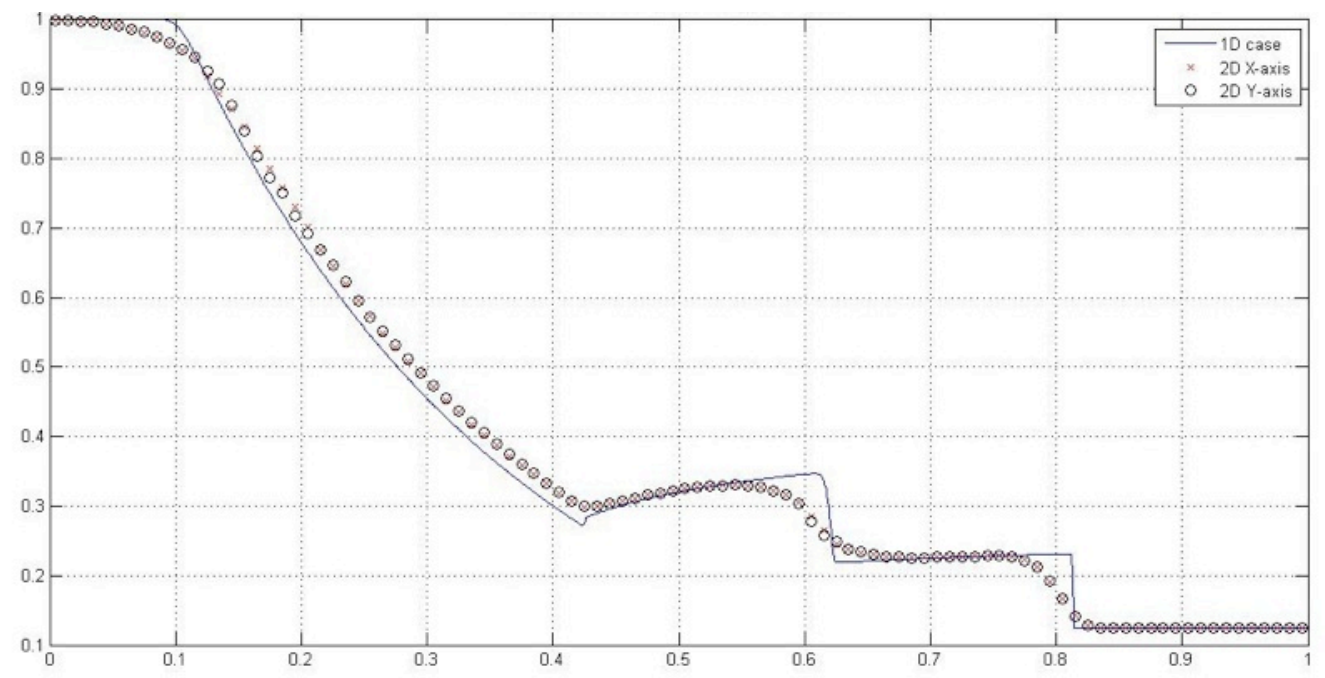

(Figure 5) Comparison of 2D and 1D solver on convection test case

The lineout shows good agreement between the two solvers. In an attempt to obtain an exact answer, the one dimensional case is run with 1500 cells. The two dimensional case is run on a mesh of $200 \times 200$ cells.

We then proceeded to simulate the setup shown in Figure 2. The initial conditions are

$$
\begin{gathered}
\rho_{i, j}= \begin{cases}1.278 & \forall i, \forall j<n \\
53.1 \quad \forall i, \forall j=n\end{cases} \\
u_{i, j}=0 \forall i, j \\
v_{i, j}=0 \forall i, j
\end{gathered}
$$

st. $r_{i, j}$ is the distance from cell $i, j$ to the center of the computational domain. The energy initial condition sets up a Gaussian temperature profile in the center of the computational domain and [5] suggested its use as a way to jump start the ohmic heating source term. Throughout the simulation run, ohmic heating was implemented with the following current profile. 


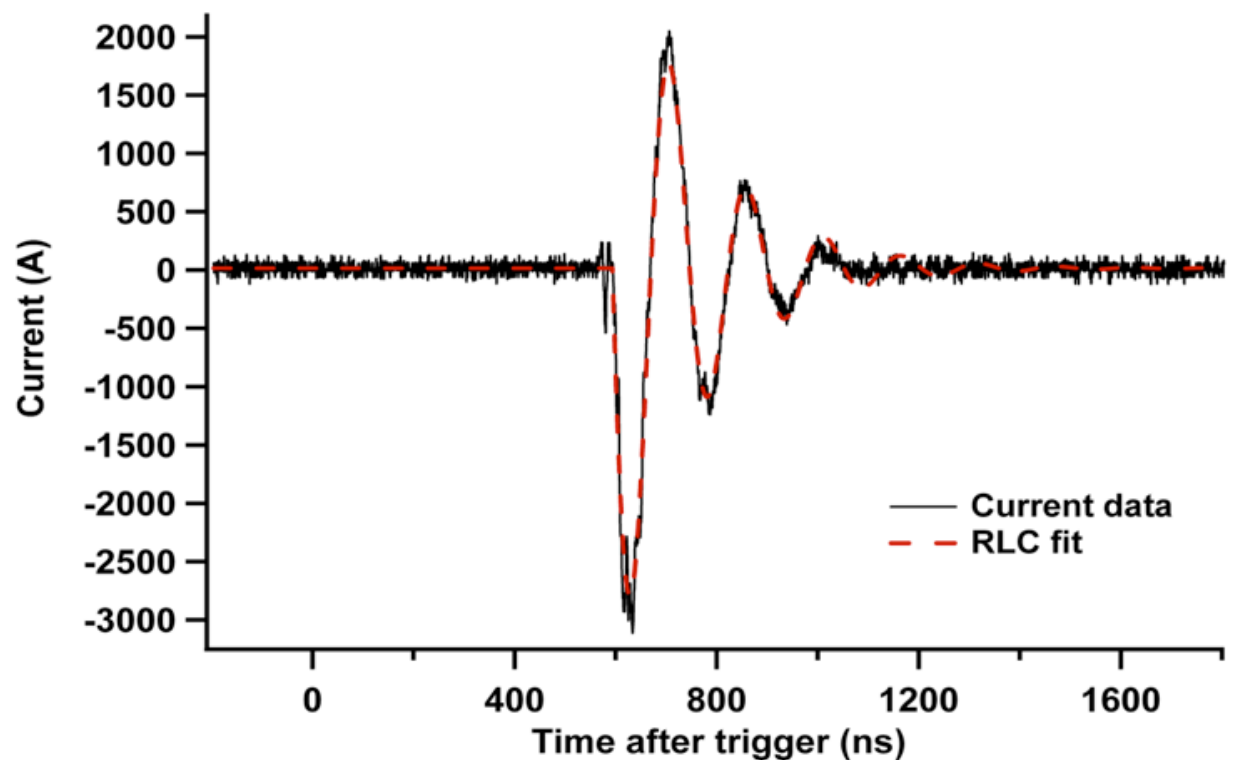

(Figure 6) Current Profile used in ohmic heating source term

The results for temperature after 1.2 microseconds are shown below.

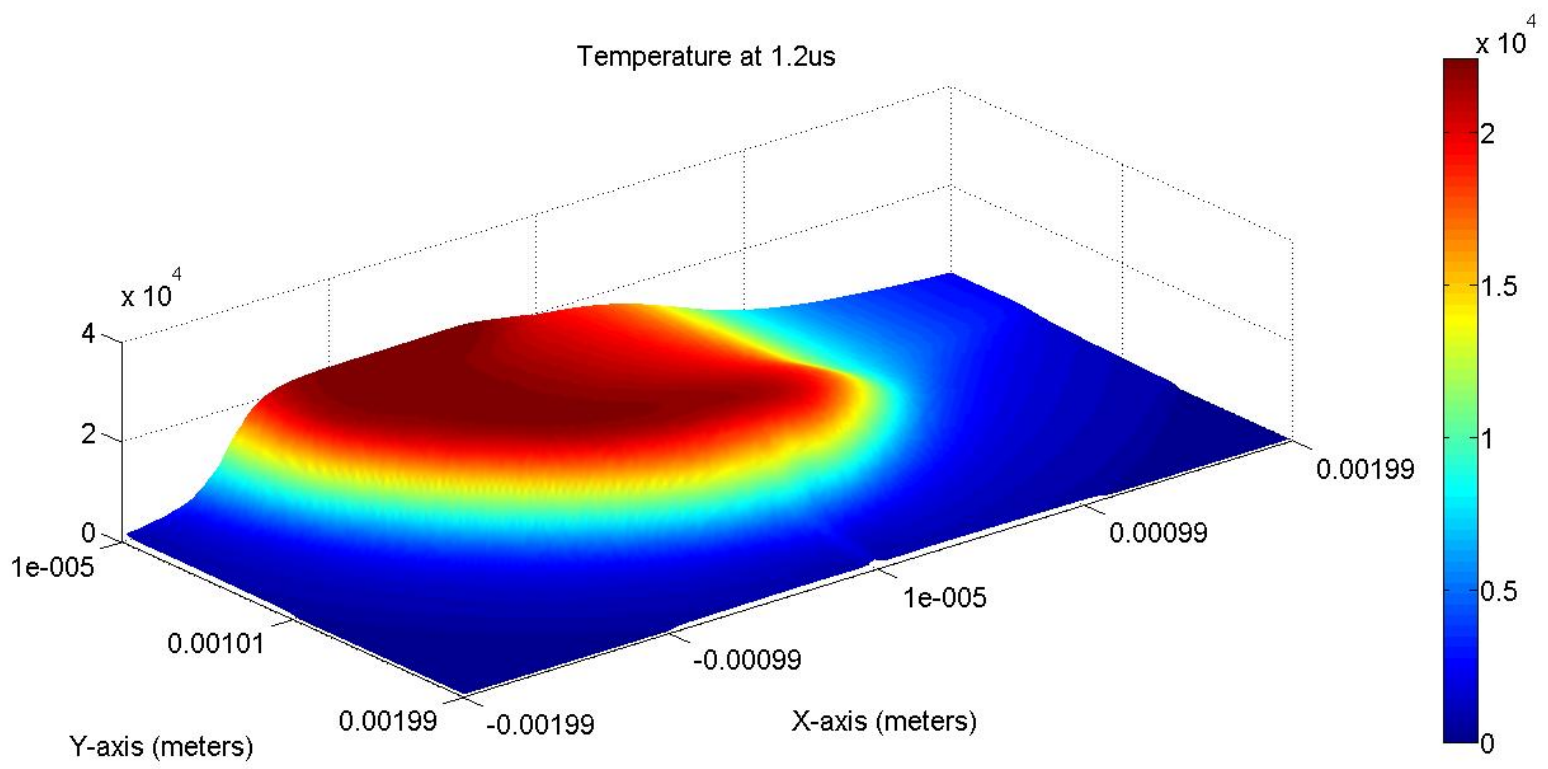

(Figure 5) Temperature Results

The asymmetry of the temperature is caused by the ohmic heating source term, which would allocate more the power to cells that were hotter than their neighbors, resulting in runaway heating of cells which obtained marginally higher temperatures than their neighbors through round off error. However, benchmarking against experimental data shows good agreement even with this numerical artifact. 


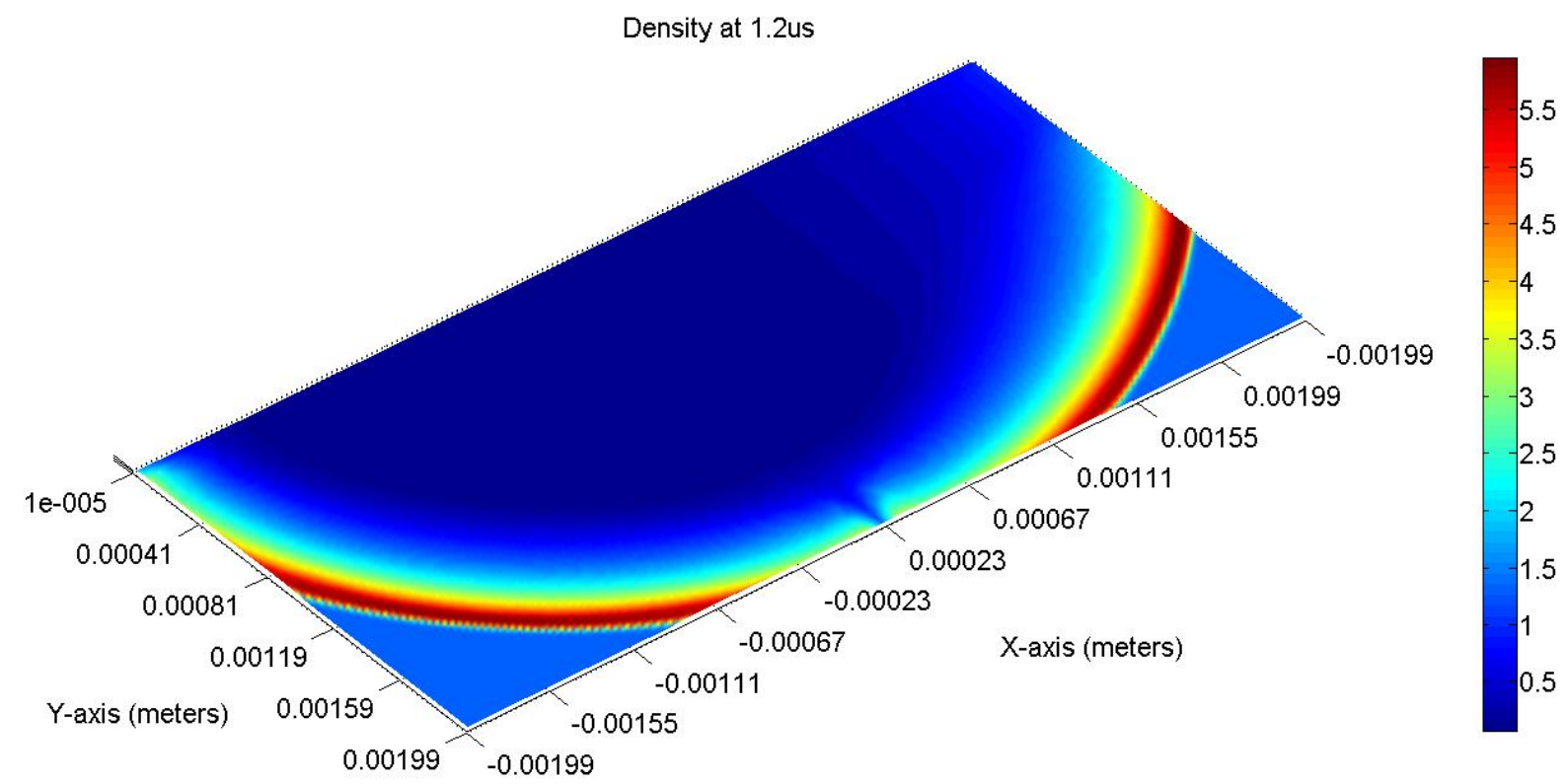

(Figure 6) Density Results

The asymmetry of density is the opposite of temperature because given similar energy densities; lower density will exhibit higher temperatures. Figure 6 also shows that the leading edge of the shock wave is much cooler than the center of the shock region.

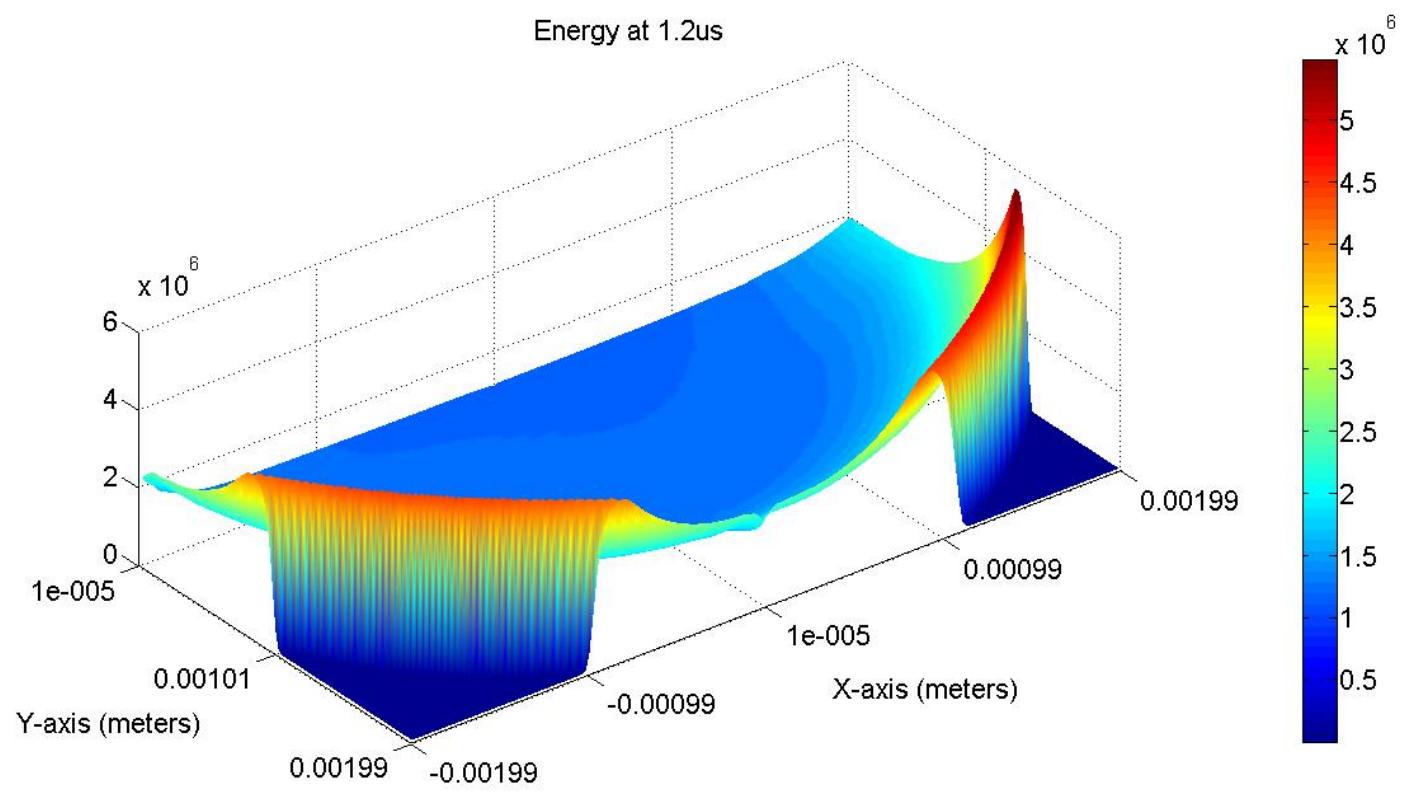

(Figure 7) Energy Results

Figure 7 explains the discrepancy between temperature radii and shock radii. While the center of the shock wave has densities roughly one order of magnitude less than the shock edge (Figure 6), their respective energy density is approximately one third as much. This discrepancy leads to particles in the shock center having three times more energy than their counterparts on the shock edge. As expected, the temperature difference in between shock center and edge is roughly a factor of three (Figure 5). 


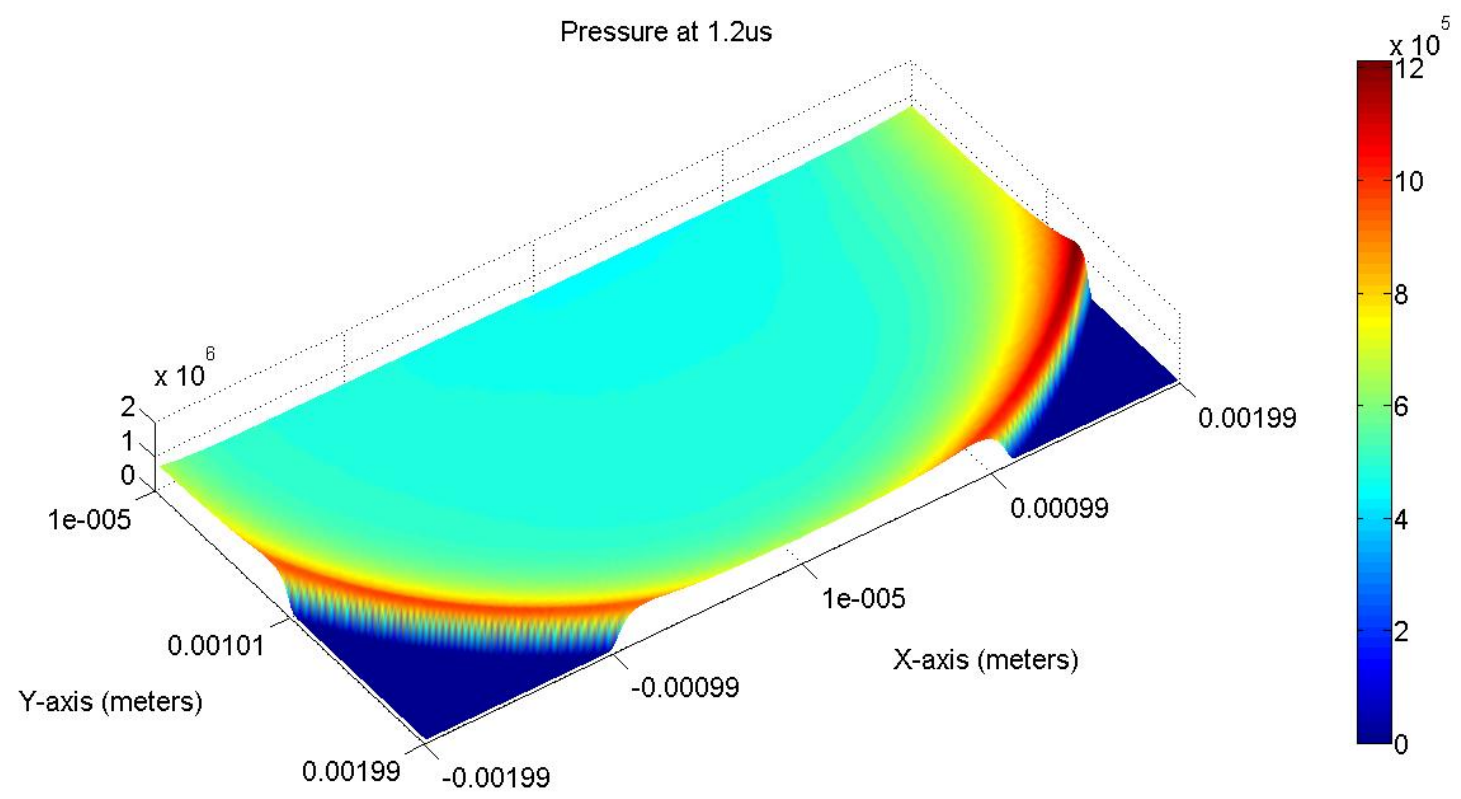

(Figure 8) Pressure Results

Figure 8 explains the asymmetry of the temperature and density terms. Since the Riemann problem is essentially pressure driven, two neighboring cells with similar pressures would experience little intercellular flux. As seen by Figure 8, runaway ohmic heating, which increased temperature and decreased density, had negligible effects on pressure. With neighboring cells in the shock center region at the same pressure, the excess energy of ohmic heating could not be dissipated through convection.

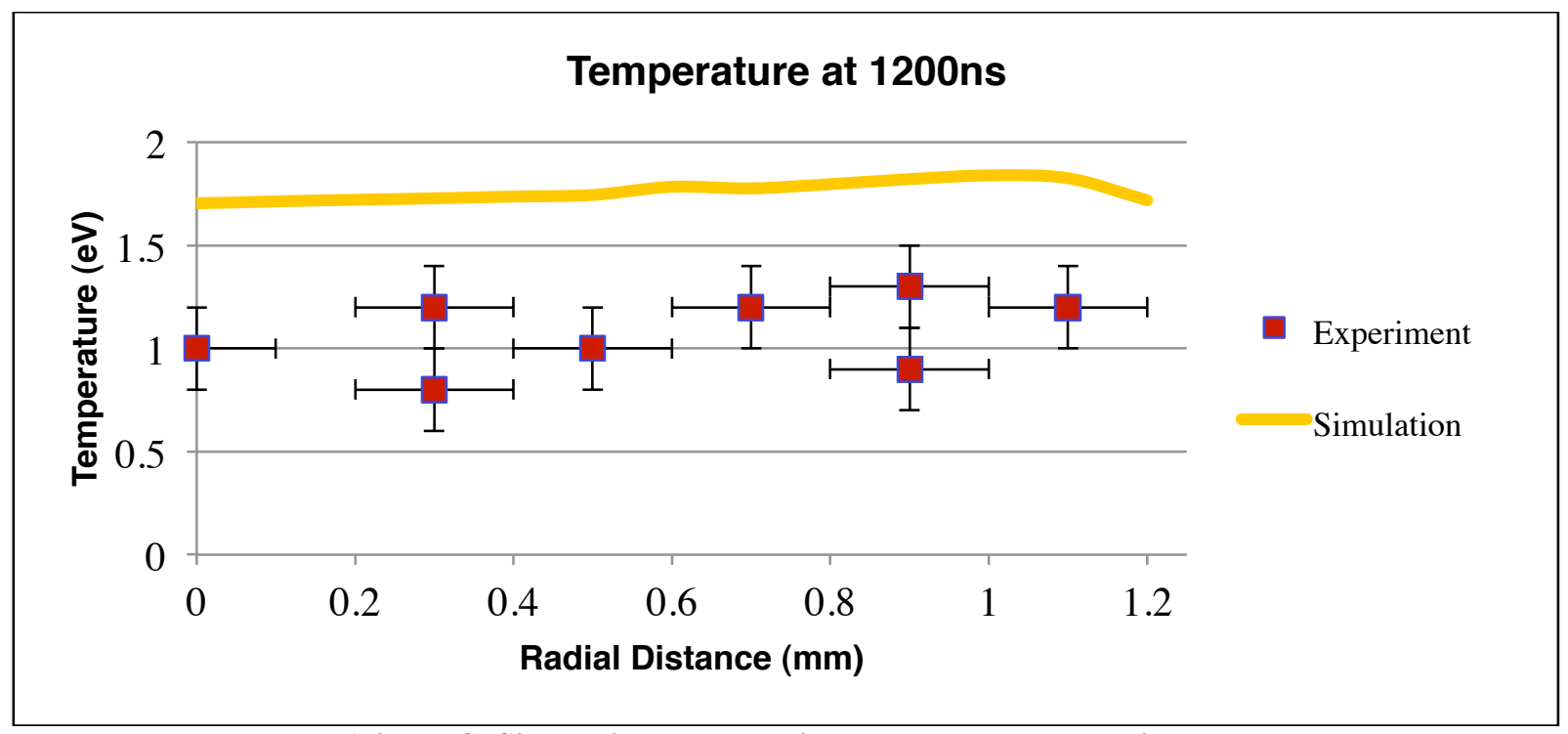

(Figure 9) Simulation vs. Experimental Temperature Lineout

Since there was asymmetry of temperature in the simulation along the $\mathrm{x}$ axis, the lineout for temperature was taken along the y axis. Because the simulation temperature is consistently 0.5 eVs higher than experimental, we believe the excess simulation temperature comes from an EOS issue. We hypothesize that an EOS which factors in the correct degrees of freedom for the fluid particles should reconcile the results. 


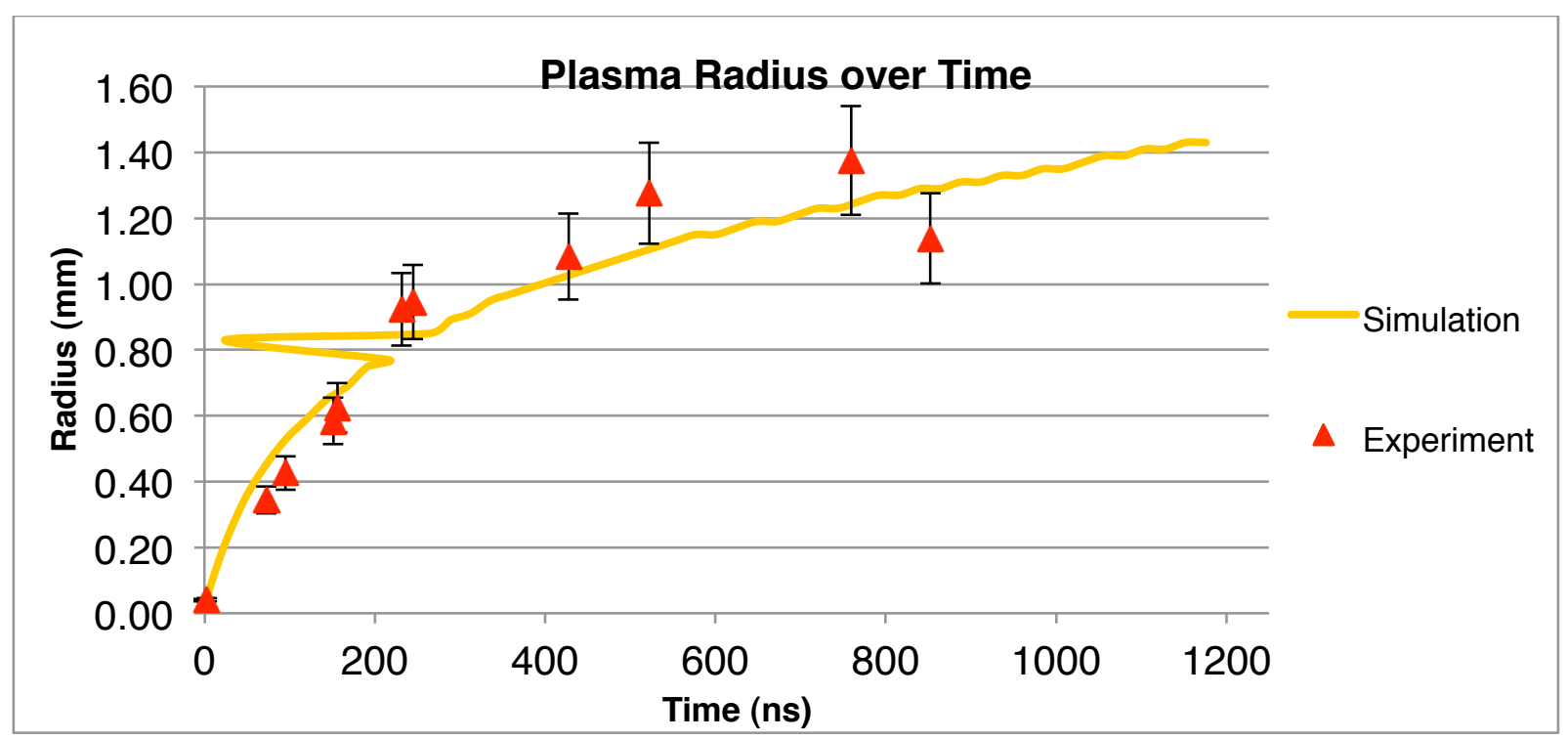

(Figure 10) Simulation vs. Experimental Radii Lineout

Similar to the temperature lineout, the radius is taken along the y axis. The radius was captured in the experiment through a visible range camera, and in the simulation, it is defined as the cell farthest from the computation domain center with $T \geq 5000 \mathrm{~K}$. The value of $5000 \mathrm{~K}$ is chosen as it represents an approximate temperature where light emission decreases significantly and as a mid-point of the steep outer temperature gradient of the simulation (Figure 6).

Initial conditions for the chemistry is $300 \mathrm{~nm}$ of PETN in gas form along the bottom of the computational domain in Y, see Figure 2. The interspecies reactions follow (22-24). After 1.2 $\mu s$, we obtained the resulting specie densities. 

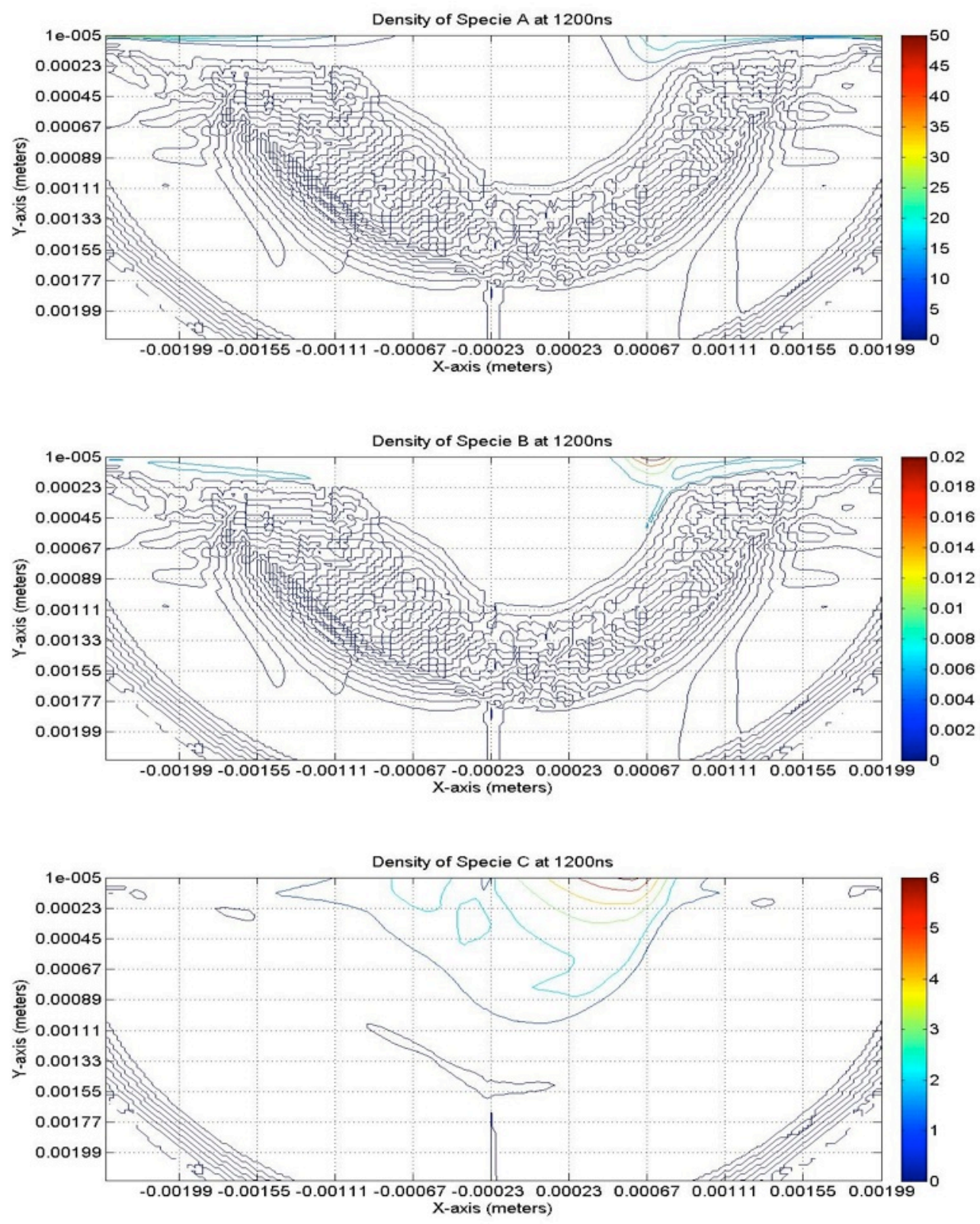

(Figure 8) Contour plots of chemical species

As expected, the individual chemical densities mirror the asymmetry of the net particle density. However, one surprising result of the chemical simulation is the minimal amount of convection of Specie C (PETN products) after their formation. This interesting result will be explored in future studies.

\section{Conclusions}

We have detailed methods for constructing a second order TVD scheme for convection equations with high gradient source terms. During development, we devoted much time to discretizing and stabilizing the first principle physics formula for the source terms. For the reader's convenience, we have a fully second order and stable scheme of the source terms including chemical reacting flows. Future work will encompass higher order solvers and even more complex multi-path reactions to simulate the entire system from first principle. 



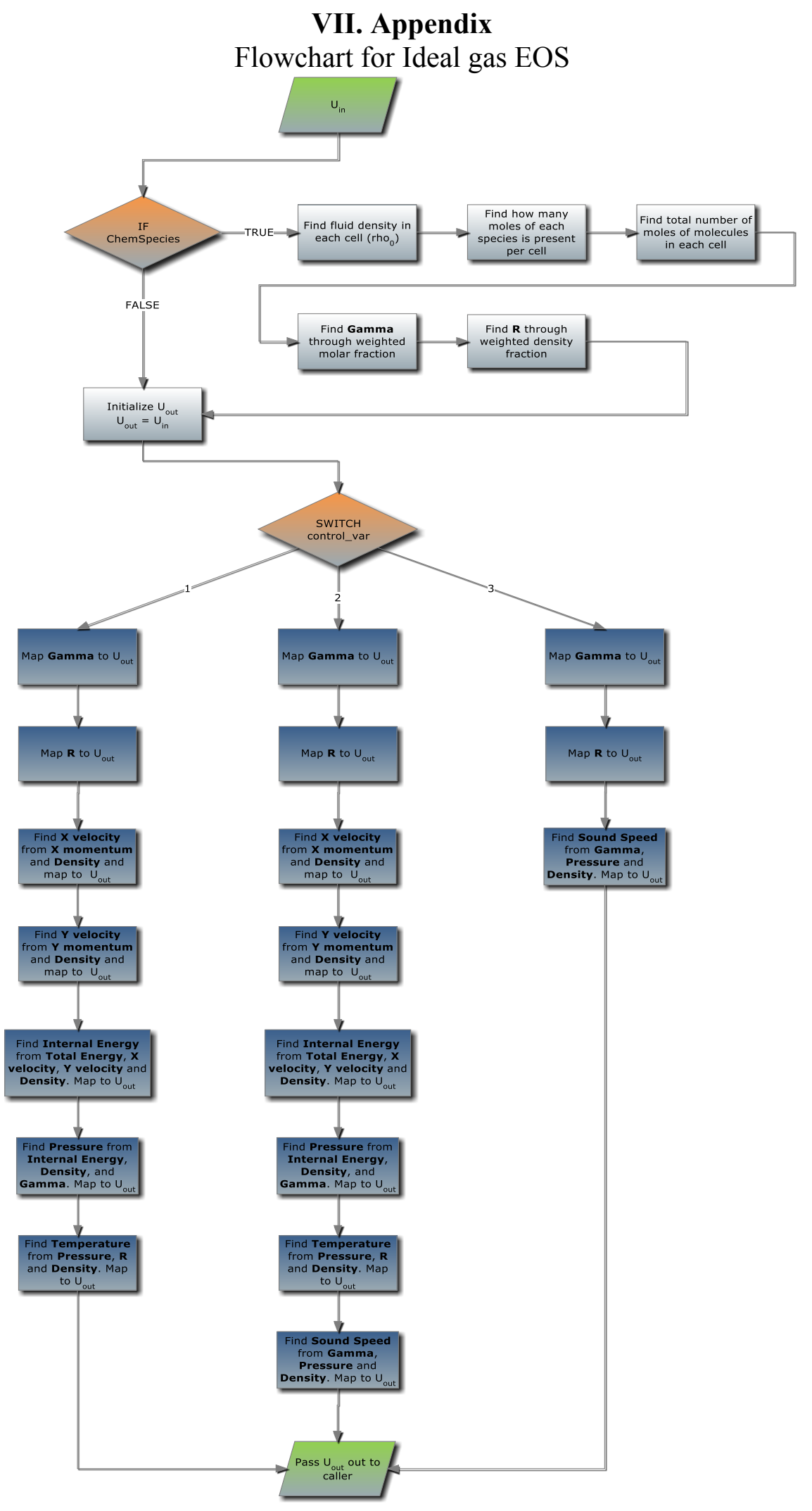

Flowchart for MUSCL-Hancock Scheme 


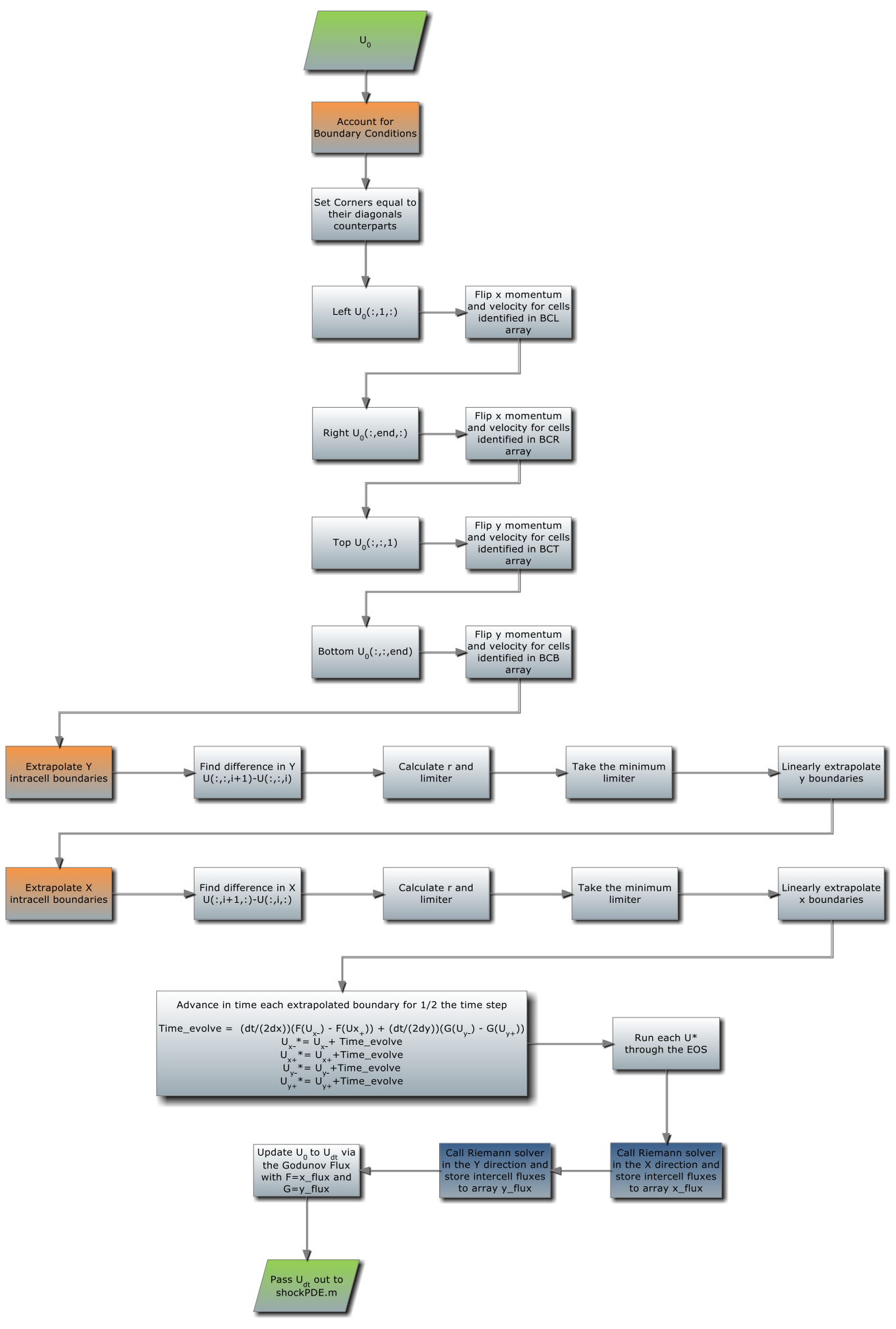

Flow chart for Source term solver 


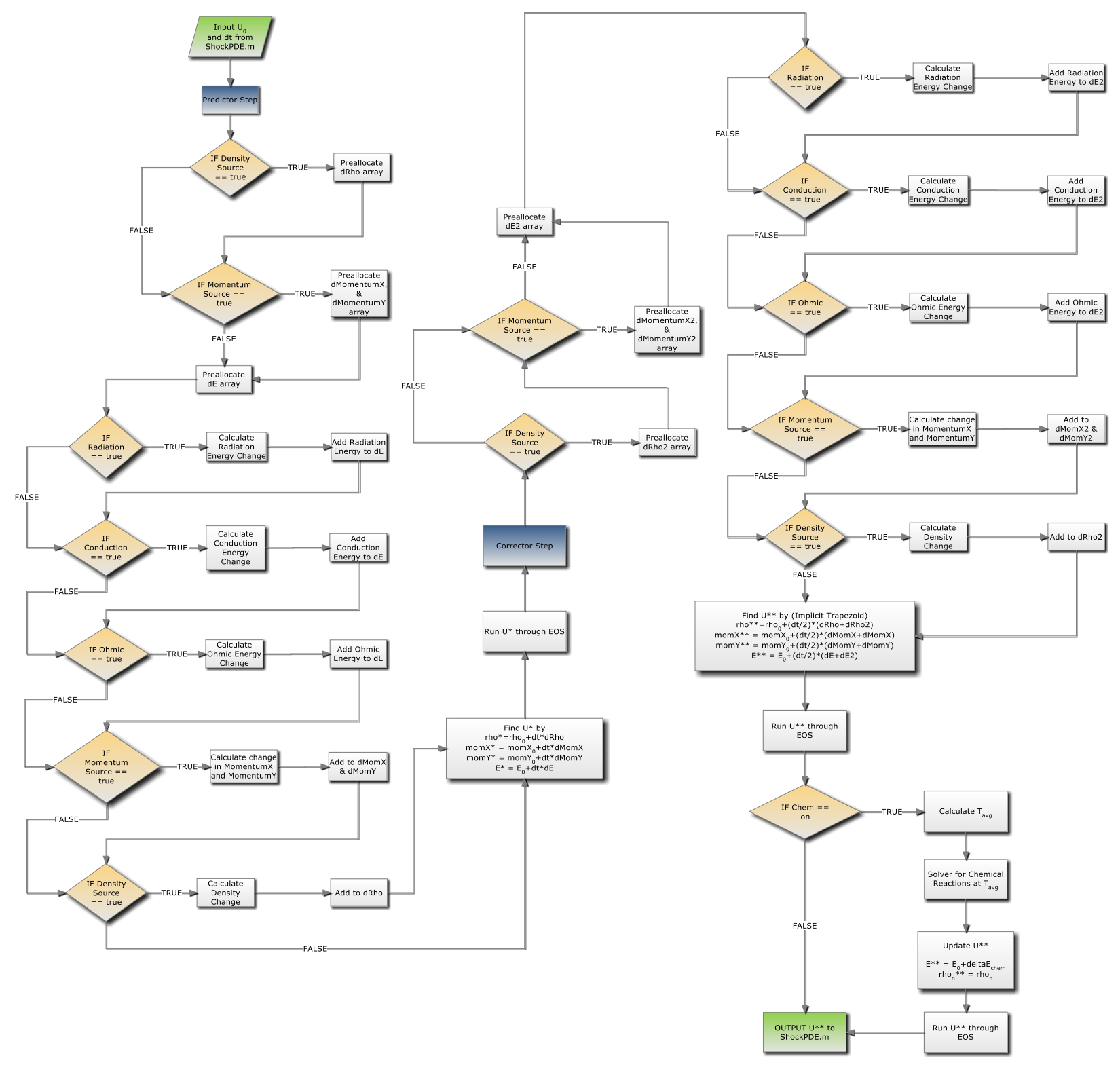




\section{References}

[1] Tucker, T. Kennedy, J., and Allensworth, D. "Secondary Explosive Spark Detonators"; Proceedings of the $7^{\text {th }}$ Symposium on Explosives and Pyrotechnics, Philadelphia, PA, 1971.

[2] Liou, W.; McCarrick, J.F., Hodgin, R., and Phillips, D. "High-Power Electrostatic Discharges in PETN: Threshold and Scaling Experiments”, 2010, Coeur D'Alene, IA.

[3] Toro E. L., Riemann Solvers and Numerical Methods for Fluid Dynamics : A Practical Introduction. 3rd. New York: Springer, 2009. 724. Print.

[4] Akram, M. "Two-Dimensional Model for Spark Discharge Simulation in Air." AMERICAN INST OF AERONAUTICS AND ASTRONAUTICS. 34. (1996): 1835-1842. Print.

[5] Plooster, Myron. "Numerical Simulation of Spark Discharges in Air." Physics of Fluids. 14.10 (1971): 2111-2124. Print.

[6] Shengtai Li, An HLLC Riemann solver for magneto-hydrodynamics, Journal of Computational Physics, v.203 n.1, p.344-357, 10 February 2005 


\section{Appendix E. MATLAB code for processing TRIR data}

function Process_TRIR_v2

$\%$ Process_TRIR_v2

$\%$ by J. M. Zaug, LLNL

\% Code to batch proces TRIR data at LLNL, B235 R1217B 3_19_2011

$\%$

$\%$ This script will take a TRIR reference file (name must be = "ref.txt")

$\%$ (air + substrate - electronic background) and compute the optical

\%absorption density (O.D.) of a time resolved single-shot IR spectrum. The

$\%$ ref.txt file must be in the same directory path as this IR_Process_TRIR_v2

$\%$ function. Each series of single shot data must be stored in folder named

\%"shot\#". Each shot folder must consist of three data files named, "Pre.txt",

\%"Trans.txt", and "Post.txt".

$\%$ The user is asked to input a folder or range of folders to process. For

$\%$ example, enter "0:4" to process folders shot0 to shot 4 .

$\%$

$\%$ Processed results are stored in each selected folder under the name

$\% "$ "folder_array" -the format is [wavenumber; Pre_OD; Trans_OD; Post_OD]

$\%$ The user may upload "complete_array" text files for further analysis.

$\%$ In addition, a running total of previous and current folder(s) processed

\%data is stored in each folder under the name "total_arrary". For example,

$\%$ if you decide to process shot 0 to shot 100 folders, then all of the

\%processed data can be found in the shot 100 folder in "total_array". Thus,

\%all processed results may be uploaded from "total_array" found in the last

$\%$ selected folder where the format is $(\mathrm{N}=$ shot $\#)$

$\%[$ wavenumberN; PreN_OD; TransN_OD; PostN_OD; wavenumberN $+1 ; \ldots$ ]

$\%$

$\%$ This script will also plot the results of each folder where curves are

$\%$ coloured as such, blue $=$ PreShot; red $=$ Transient; green $=$ PostShot. For

$\%$ clarity, each dataset is offset in the plot by an O.D. value of 1.00 .

$\%$

clear; close all

format long; warning off MATLAB:divideByZero

program_name='Process_TRIR_v2';

Dpath = which(eval(['program_name'])); \%Finds the entire path to the current data

ncs $=0 ; \% \#$ of characters to the right of the decimal pt.

\%including the decimal pt. (e.g., '.txt.10', ncs =7)

$\%$ Assumes you're running from a directory containing reference spectra

shifter=1.00; \%Amount to shift spectra on same plot (units = O.D.)

$\%$ cd 'C: $\backslash$ Documents and Settings $\backslash z a u g 1 \backslash$ Application Data $\backslash$ Desktop $\backslash R$ projects $\backslash$ PETNtrir_2';

$\%$ Dpath $=$ 'C: $\backslash$ Documents and Settings $\backslash$ zaug $1 \backslash$ Application Data $\backslash$ Desktop $\backslash I R$ projects $\backslash P E T N$ trir_2';

$\%$ Enter in the pot. range of TDIR folders

tdirs=\{'shot0';'shot1';'shot2';'shot3';'shot4';'shot5';'shot6';'shot7';'shot8';'shot9';'shot10'; 
'shot11';'shot12';'shot13';'shot14';'shot15';'shot16';'shot17';'shot18';'shot19';'shot20'; 'shot21';'shot22';'shot23';'shot24';'shot25';'shot26';'shot27';'shot28';'shot29';'shot30'; 'shot31';'shot32';'shot33';'shot34';'shot35';'shot36';'shot37';'shot38';'shot39';'shot40'; 'shot41';'shot42';'shot43';'shot44';'shot45';'shot46';'shot47';'shot48';'shot49';'shot40'; 'shot51';'shot52';'shot53';'shot54';'shot55';'shot56';'shot57';'shot58';'shot59';'shot60'; 'shot61';'shot62';'shot63';'shot64';'shot65';'shot66';'shot67';'shot68';'shot69';'shot70'; 'shot71';'shot72';'shot73';'shot74';'shot75';'shot76';'shot77';'shot78';'shot79';'shot80'; 'shot81';'shot82';'shot83';'shot84';'shot85';'shot86';'shot87';'shot88';'shot89';'shot90'; 'shot91';'shot92';'shot93';'shot94';'shot95';'shot96';'shot97';'shot98';'shot99';'shot100';

'shot101';'shot102';'shot103';'shot104';'shot105';'shot106';'shot107';'shot108';'shot109';'shot110'; 'shot111';'shot112';'shot113';'shot114';'shot115';'shot116';'shot117';'shot118';'shot119';'shot120'; 'shot121';'shot122';'shot123';'shot124';'shot125';'shot126';'shot127';'shot128';'shot129';'shot130'; 'shot131';'shot132';'shot133';'shot134';'shot135';'shot136';'shot137';'shot138';'shot139';'shot140'; 'shot141';'shot142';'shot143';'shot144';'shot145';'shot146';'shot147';'shot148';'shot149';'shot150'; 'shot151';'shot152';'shot153';'shot154';'shot155';'shot156';'shot157';'shot158';'shot159';'shot160'; 'shot161';'shot162';'shot163';'shot164';'shot165';'shot166';'shot167';'shot168';'shot169';'shot170'; 'shot171';'shot172';'shot173';'shot174';'shot175';'shot176';'shot177';'shot178';'shot179';'shot180'; 'shot181';'shot182';'shot183';'shot184';'shot185';'shot186';'shot187';'shot188';'shot189';'shot190'; 'shot191';'shot192';'shot193';'shot194';'shot195';'shot196';'shot197';'shot198';'shot199';'shot200';\};

$\%$ ltdirs $=$ length(tdirs); $\%$ \# of TRIR files

$\%$ nums $=$ cell(ltdirs, 1$)$

$\%$ for $\mathrm{p}=1: 1$ tdirs nums $\{\mathrm{p}\}=\mathrm{p}$; end $\%$ ndirectories $=[$ nums tdirs]; $\%$ show user all of the entered directories and their assigned \#

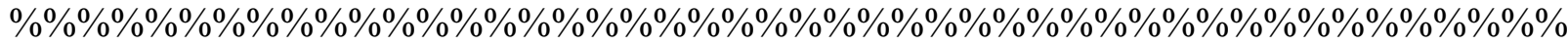
\%\%\%\%\%\%\%\%\%\%\%\%\%\%\%\%\%\%\%\%\%\%\%\%\%\%\%\%\%\%\%\%\%\%\%\%\%

ref = 'ref.txt'; \% Common name for all TRIR reference data; length of file does not matter ref_file $=$ load(ref); reference_data $=$ ref_file;

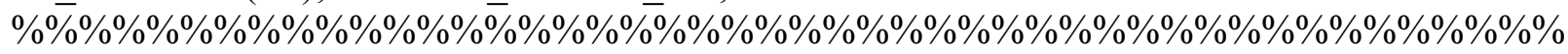
\%\%\%\%\%\%\%\%\%\%\%\%\%\%\%\%\%\%\%\%\%\%\%\%\%\%\%\%\%\%\%\%\%\%\%\%\%

$\%$ Load up n-shots of Ref data and compute the average

$[$ rowr,colr $]=$ size $($ reference_data);

if colr $>=3$ data_avgr=zeros(rowr, 1$)$; \%preload data array for $\mathrm{pdr}=2: 1:$ colr; data_pdr=reference_data(:,pdr); 


$$
\text { end }
$$

data_avgr=[data_avgr + data_pdr $] ; \%$ add up the $n$-shots

REFDATAcol2 = data_avgr / colr; \%average over n-shots

$$
\text { end }
$$

wavenumber=reference_data(:,1);

$\mathrm{REF}=\mathrm{REFDATAcol} 2$;

$\operatorname{disp}('$ ' $)$

number=input('Enter Shot Folder(s) to Process " \# or \#,\# or \#:\# etc. " ');

min_num $=\min ($ number $)+1 ; \%$ add one since MatLab vector lengths start at one, not zero!

max_num $=\max ($ number $)+1$;

\%up to 3 possible color plots should be a sufficient range. color=[ 'b' 'r' 'g'];

$\%$ color $=$ [color color]; color $=[$ color color $]$; color $=[$ color color $]$; color $=[$ color color $]$; color $=[$ color color $]$; color $=[$ color color $]$; color $=[$ color color $]$; color $=[$ color color $]$; color $=[$ color color $]$; color $=[$ color color $]$;

$\%$ color $=[$ color color $]$; color $=[$ color color $]$; color $=[$ color color $]$; color $=[$ color color $]$; color $=[$ color color $]$; color $=[$ color color $]$; color $=[$ color color $]$; color $=[$ color color $]$; color $=[$ color color $]$; color $=[$ color color $]$;

$\%$ Change to Data directory

\%Dpath='C: Documents and Settings $\backslash$ zaug $1 \backslash A$ pplication Data $\backslash$ Desktop $\backslash$ IR projects $\backslash P E T N t r i r ' ;$ scrsz $=\operatorname{get}(0$, 'ScreenSize');

$\%$ (units in pixels) left bottom width height

figure('Position',[1 $\operatorname{scrsz}(4) / 2 \operatorname{scrsz}(3) / 2 \quad \operatorname{scrsz}(4) / 2])$;

$\%$

total_data $=[] ;$ icounter $=0$;

for $\mathrm{j}=$ min_num : $1: \max \_$num; $\%$ Start the process folder data loop icounter $=$ icounter $+1 ; \%$ Loop iteration counter;

folder_data $=[] ; \%$ Reset or clear the data array to be saved

\%load the input files;

program_path $=$ which $($ eval $([$ 'program_name'] $)) ;$ lpn=length $($ program_name $)+2$;

folders_path = program_path(1:length(program_path) - lpn);

$\mathrm{pln}=\mathrm{j}$;

newpath $=$ [folders_path, '।' tdirs $\{p \ln \}]$;

cd(newpath); \%Change the path to the correct shot folder

load Pre.txt-ASCII; \%Ir pre shot data within specified data folder load Trans.txt -ASCII; \%Ir transient shot data within specified data folder

load Post.txt -ASCII; \%Ir post shot data within specified data folder

DATA_PRE=Pre; DATA_TRANS=Trans(:,2); DATA_POST $=$ Post; \% Fix the trans data to just the 2 nd column

$\%$ Load up n-shots of sample data

[row_pre,col_pre]=size(DATA_PRE);[row_trans,col_trans]=size(DATA_TRANS);[row_post,co 1_post] $=$ size(DATA_POST); 


\section{$\%$ The next}

if col_pre $>=3 \%$ Start pre shot data averaging

data_pre_avg=zeros(row_pre,1); \%preload pre data array

for $\mathrm{pd}=2: 1: \mathrm{col} \_$pre;

data_pre_pd=DATA_PRE(:,pd);

data_pre_avg=[data_pre_avg + data_pre_pd]; \%add up the n-shots

end

DATA_PRE = data_pre_avg / col_pre; \%average over n-shots

end $\quad \%$ End pre shot data averaging

if col_post $>=3 \%$ Start post shot data averaging

data_post_avg=zeros(row_post,1); \%preload post data array

for $\mathrm{pd}=2: 1:$ col post;

data_post_pd=DATA_POST(:,pd);

data_post_avg=[data_post_avg + data_post_pd]; \%add up the n-shots end

DATA_POST $=$ data_post_avg / col_post; \%average over n-shots

end $\% \%$ End post shot data averaging

$\%$ Process data

AB_pre $=\log 10$ (REF./DATA_PRE);

AB_trans $=\log 10($ REF./DATA_TRANS);

AB_post $=\log 10($ REF./DATA_POST $)$;

$\%$ Plot the results

$\%$ if icounter $==1$ shifter $=0$; end

plot(wavenumber, $A B \_$pre $+($shifter $+($icounter- 2$))$, 'b'); if icounter $==1$ hold; grid; end

plot(wavenumber, AB_trans $+($ shifter $+($ icounter- 2$))$, 'r');

plot(wavenumber, AB_post $+($ shifter $+($ icounter-2) $)$, ' $\mathrm{g}$ ');

$\% \mathrm{Abs}=\mathrm{AB}$

$\%$

$\%$ Save routine

$\%$ cd(newpath);

folder_data $=$ [folder_data wavenumber AB_pre $\mathrm{AB} \_$trans $\mathrm{AB} \_$post $]$;

total_data $=$ [total_data folder_data];

save ('folder_array', 'folder_data', '-ASCII')

save ('total_array', 'total_data', '-ASCII')

end $\%$ END the process folder data loop

ylabel('ABS O. D.'); xlabel('Wavenumber, $\mathrm{cm}^{\wedge}{ }^{\wedge}{ }^{1}$ ');

hold off

complete_array=[wavenumber total_data];

[cola, rowa $=$ size $($ complete_array);

$\% \% \% O L D$ Code for v1 program...NOT USED/REQUIRED in V2 !!! 
$\% \% \%$--- These next lines assume multiple data has been loaded e.g. pre trans post --- \%\%\%

$\%$ Here we perform rudimentary anlysis and plots; then save all of the results

$\% \quad$ left bottom width height

\%figure('Position',[600 scrsz(4)/2 scrsz(3)/1.84 scrsz(4)/1.1]);

blank_row=zeros(cola, 1$) ; \%$ make a row of zeros to demark ABS data (pre, trans, post) by analysis result rows

if rowa $==4$

$\% \quad$ left bottom width height

figure('Position',[600 scrsz(4)/2 scrsz(3)/1.84 scrsz(4)/1.1]);

dABS_pre $=$ complete_array $(:, 2)$ - complete_array $(:, 3) ; \quad \%$ pre - trans

subplot(2,2,1); plot(wavenumber, dABS_pre); ylabel('pre - trans'); xlabel('Wavenumber, $\mathrm{cm}^{\wedge}$ -

${ }^{\prime} 1$ ); axis tight

PreABS_ratio $=$ complete_array(:,3)./complete_array(:,2); $\%$ trans / pre

subplot $(2,2,2)$; plot(wavenumber, PreABS_ratio);ylabel('trans / pre'); xlabel('Wavenumber, $\left.\mathrm{cm}^{\wedge}-^{\wedge} 1^{\prime}\right)$;axis tight

$\mathrm{dABS}=$ complete_array $(:, 2)$ - complete_array(:,4); $\%$ pre - post

subplot(2,2,3); plot(wavenumber, dABS); ylabel('pre - post'); xlabel('Wavenumber, $\mathrm{cm}^{\wedge}$ $\wedge^{\prime}$ ');axis tight

PostABS_ratio= complete_array(:,4)./complete_array(:,2); \% post / pre

subplot $(2,2,4)$; $\operatorname{plot}($ wavenumber, PostABS_ratio); ylabel('post / pre'); xlabel('Wavenumber, $\left.\mathrm{cm}^{\wedge}-1^{\prime}\right)$; axis tight

data_diffs_ratios $4=$ [blank_row dABS_pre PreABS_ratio dABS PostABS_ratio];

\%wavenumber pre trans post zeros pre-trans trans/pre pre-post post/pre

complete_array $=[$ complete_array data_diffs_ratios 4$]$;

elseif rowa $==3$

figure('Position',[600 scrsz(4)/2 scrsz(3)/1.84 scrsz(4)/1.1]);

dABS_pre $=$ complete_array $(:, 2)$ - complete_array $(:, 3) ; \quad \%$ pre - trans

subplot(2,1,1); plot(wavenumber, dABS_pre); ylabel('pre - trans'); xlabel('Wavenumber, $\mathrm{cm}^{\wedge}$ ${ }^{\wedge} 1$ '); axis tight

PreABS_ratio $=$ complete_array(:,3)./complete_array $(:, 2) ; \%$ trans $/$ pre

subplot $(2,1,2)$; $\operatorname{plot}($ wavenumber, PreABS_ratio);ylabel('trans / pre'); xlabel('Wavenumber, $\mathrm{cm}^{\wedge} \_\wedge 1$ ); ; axis tight

data_diffs_ratios $3=$ [blank_row dABS_pre PreABS_ratio];

\%wavenumber pre trans post zeros pre-trans trans/pre

complete_array=[complete_array data_diffs_ratios3];

end

\%save ('complete_array', 'complete_array', '-ASCII') 


\section{Appendix F. Drawings of Clamp and Holder Hardware}

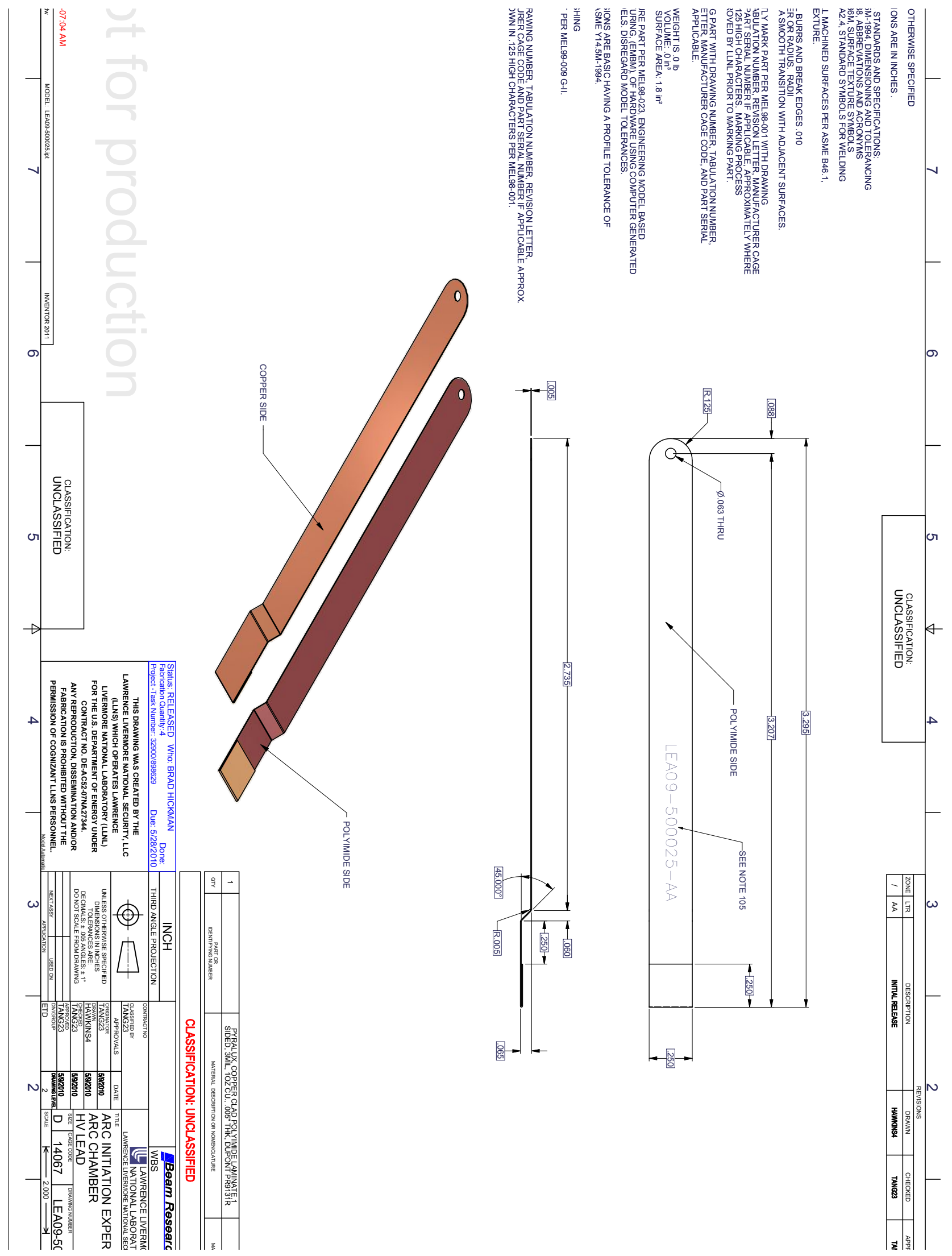



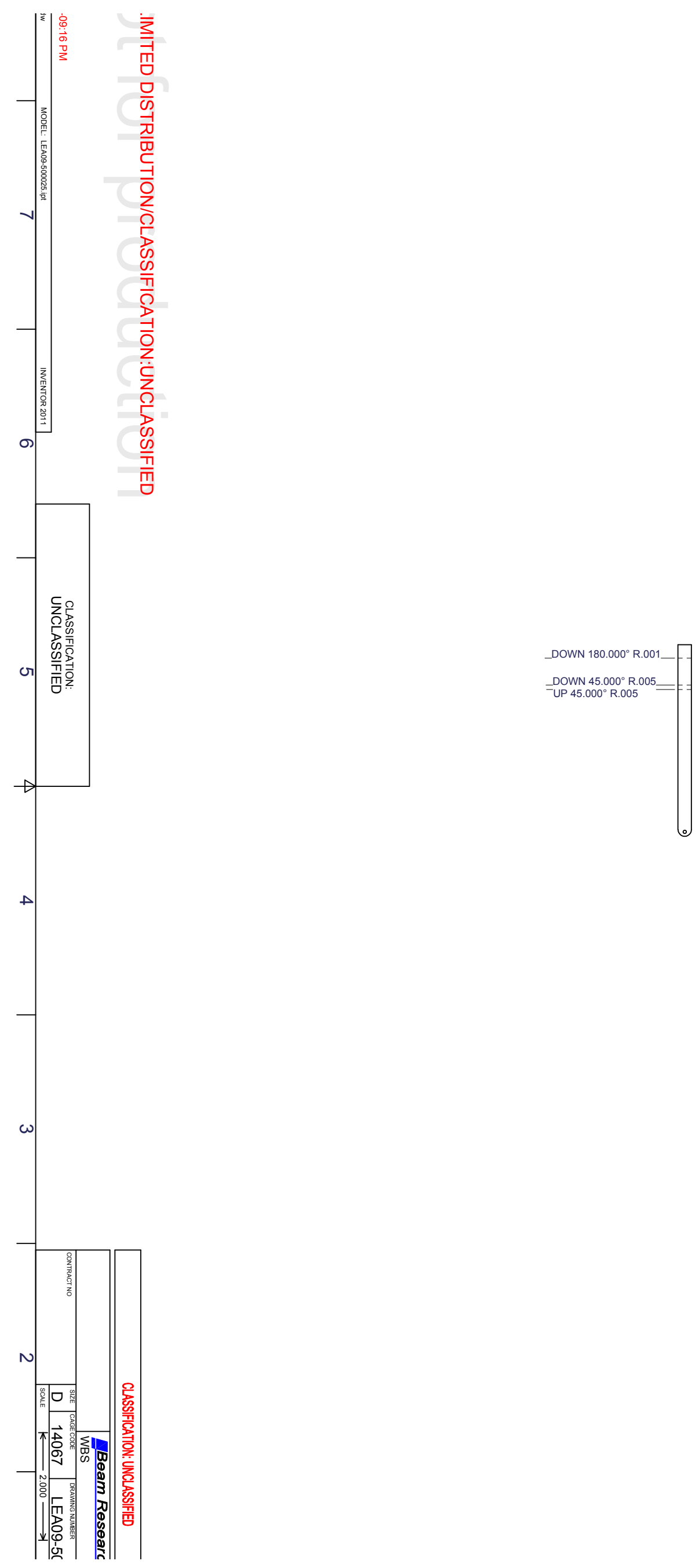

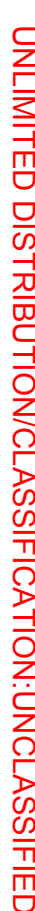




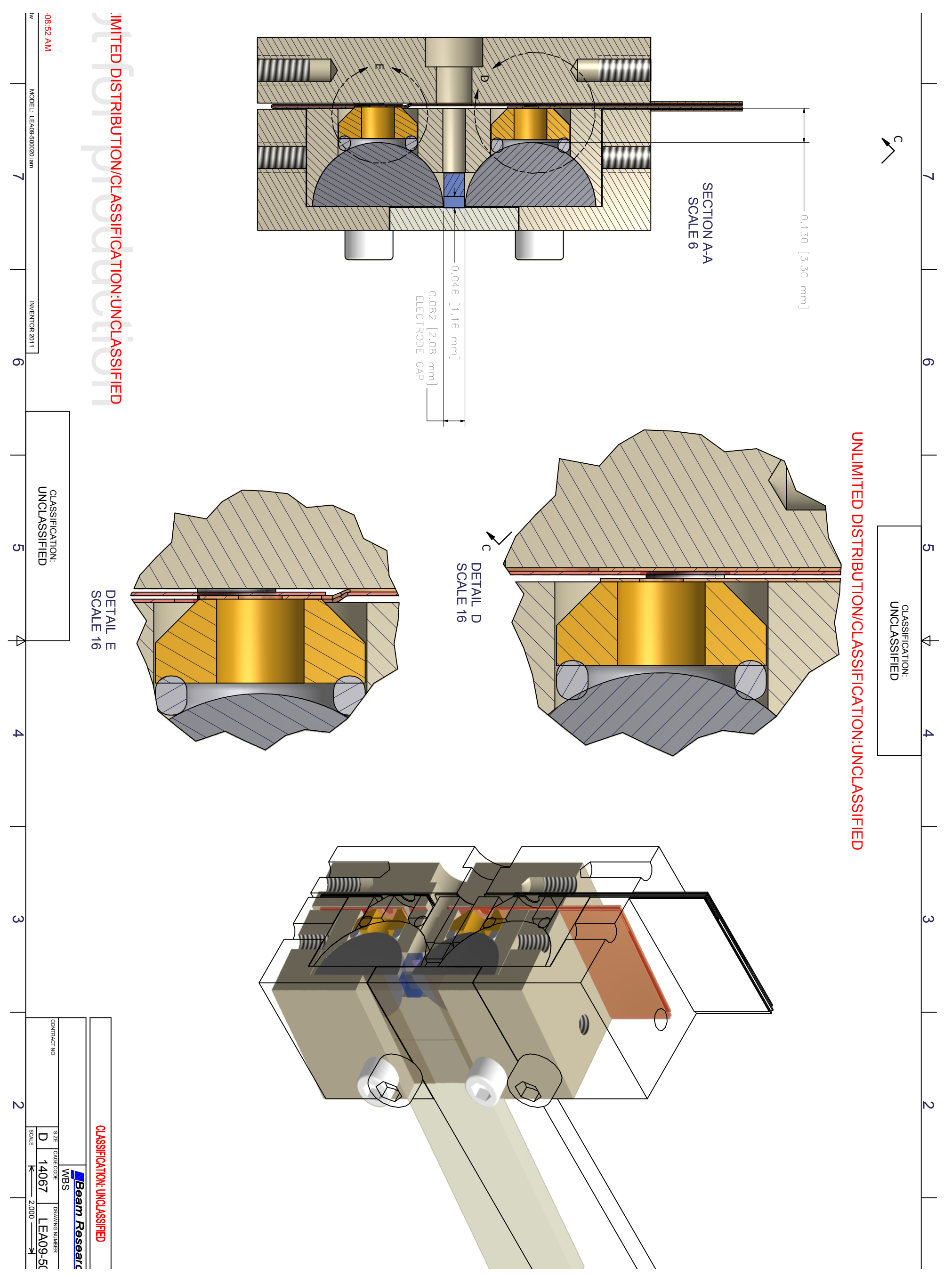




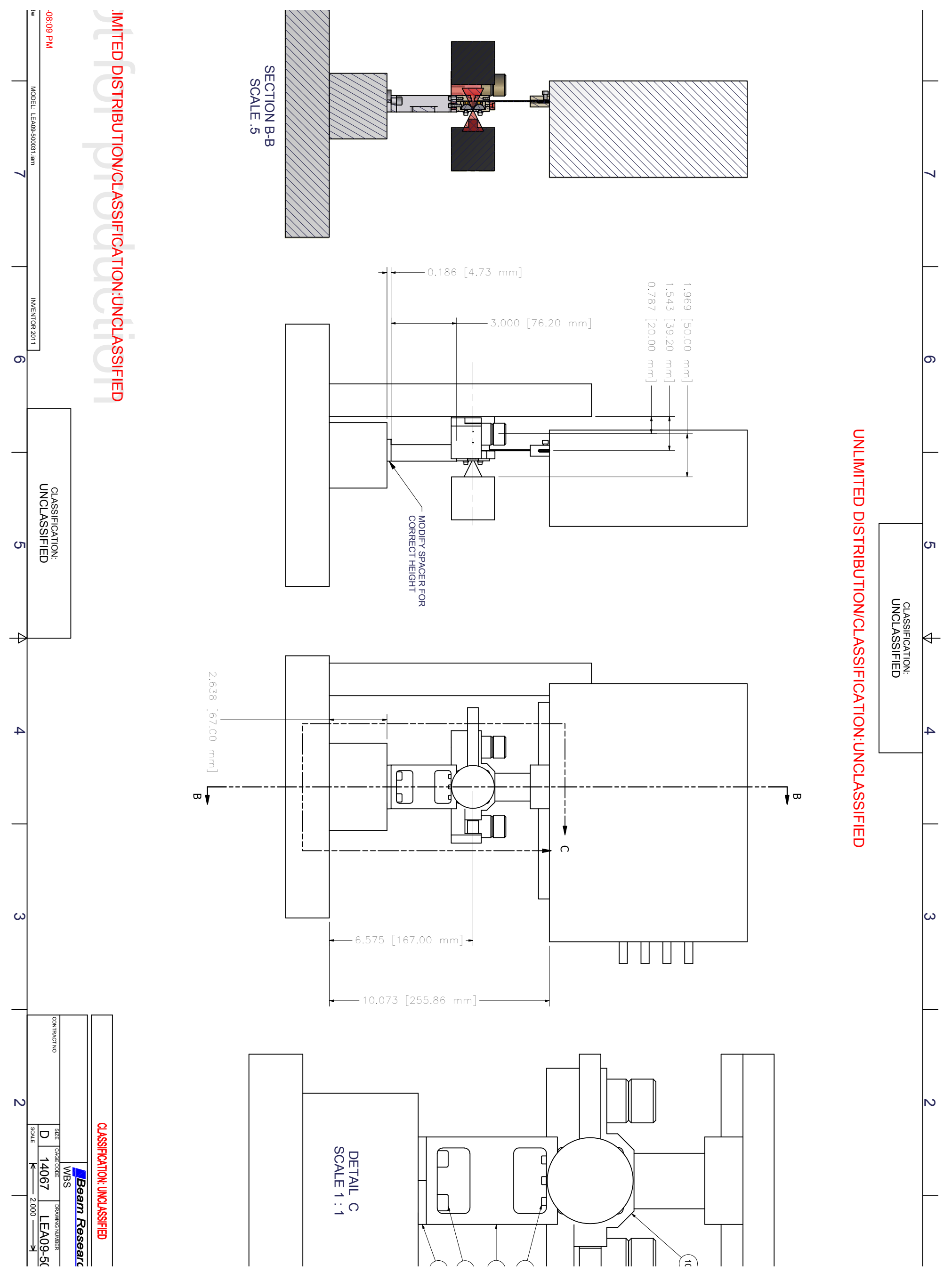




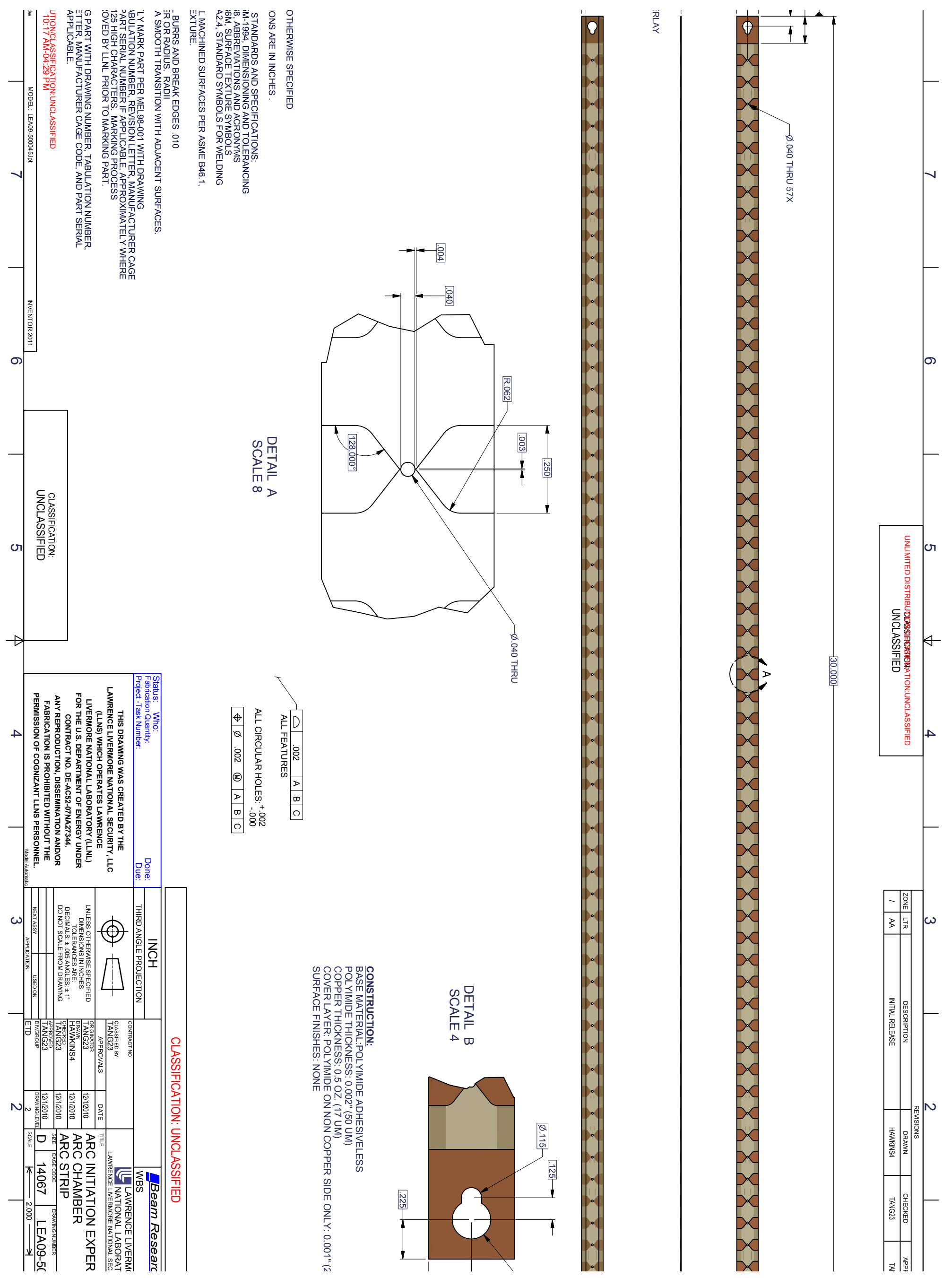



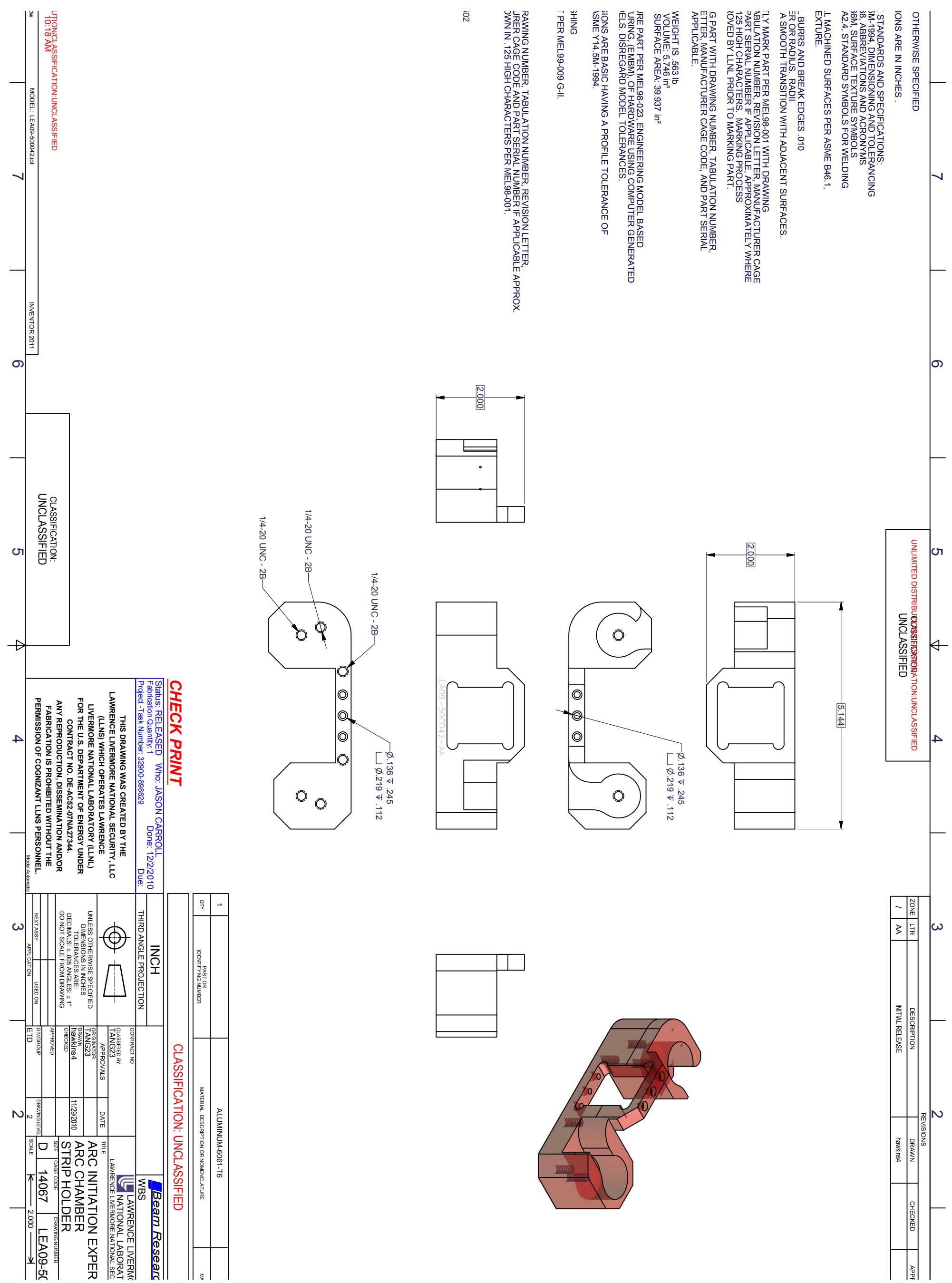


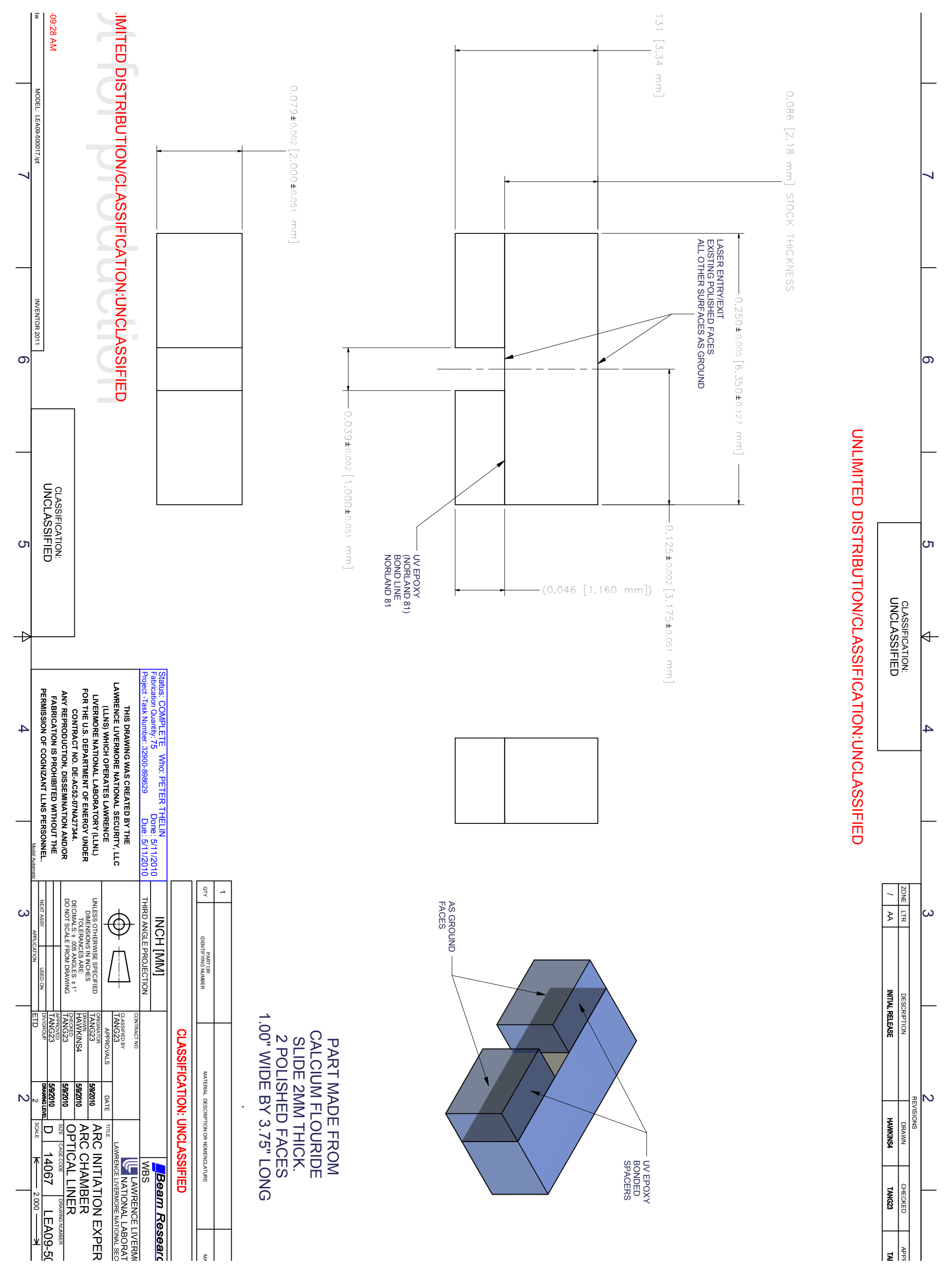




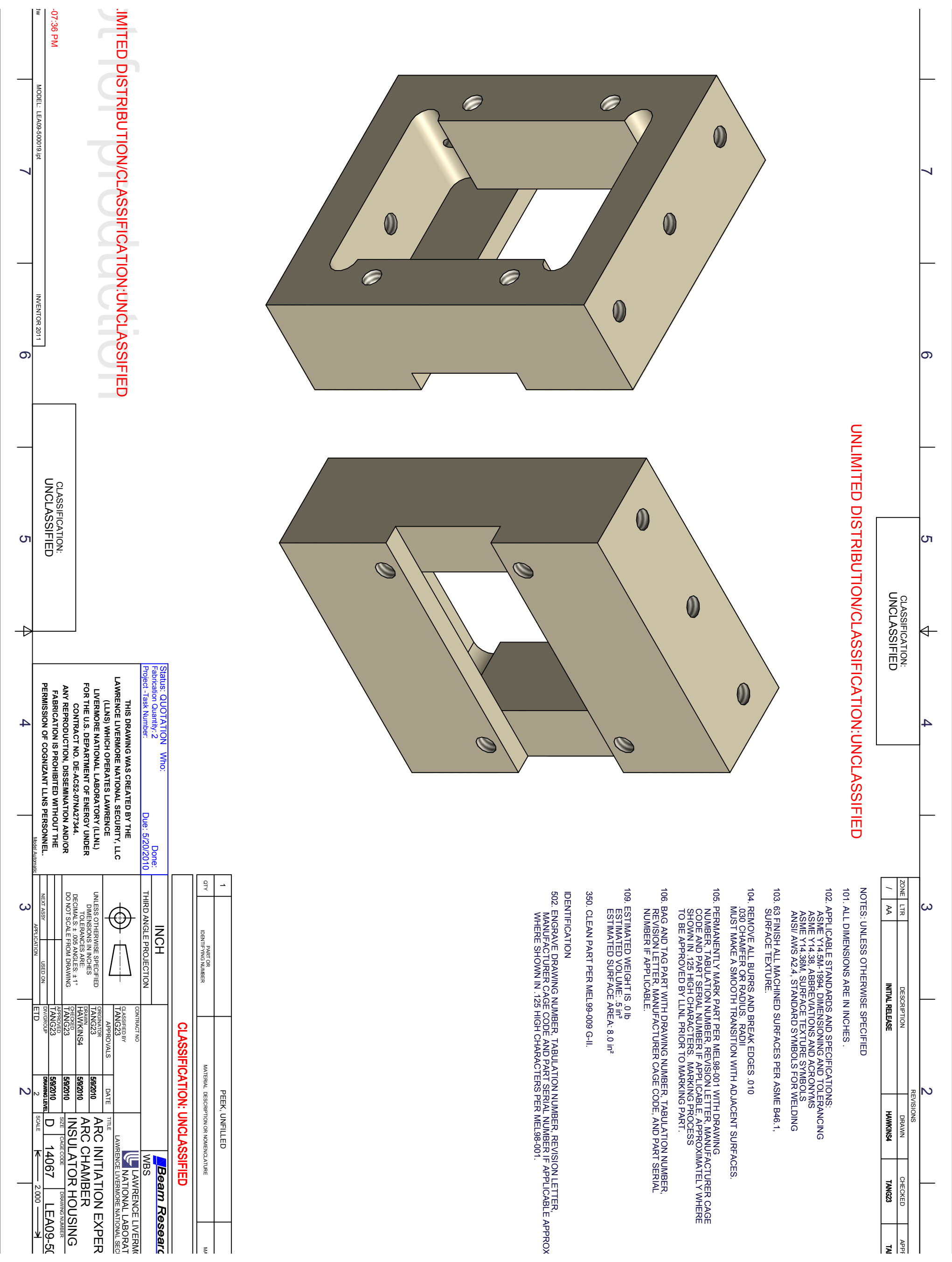




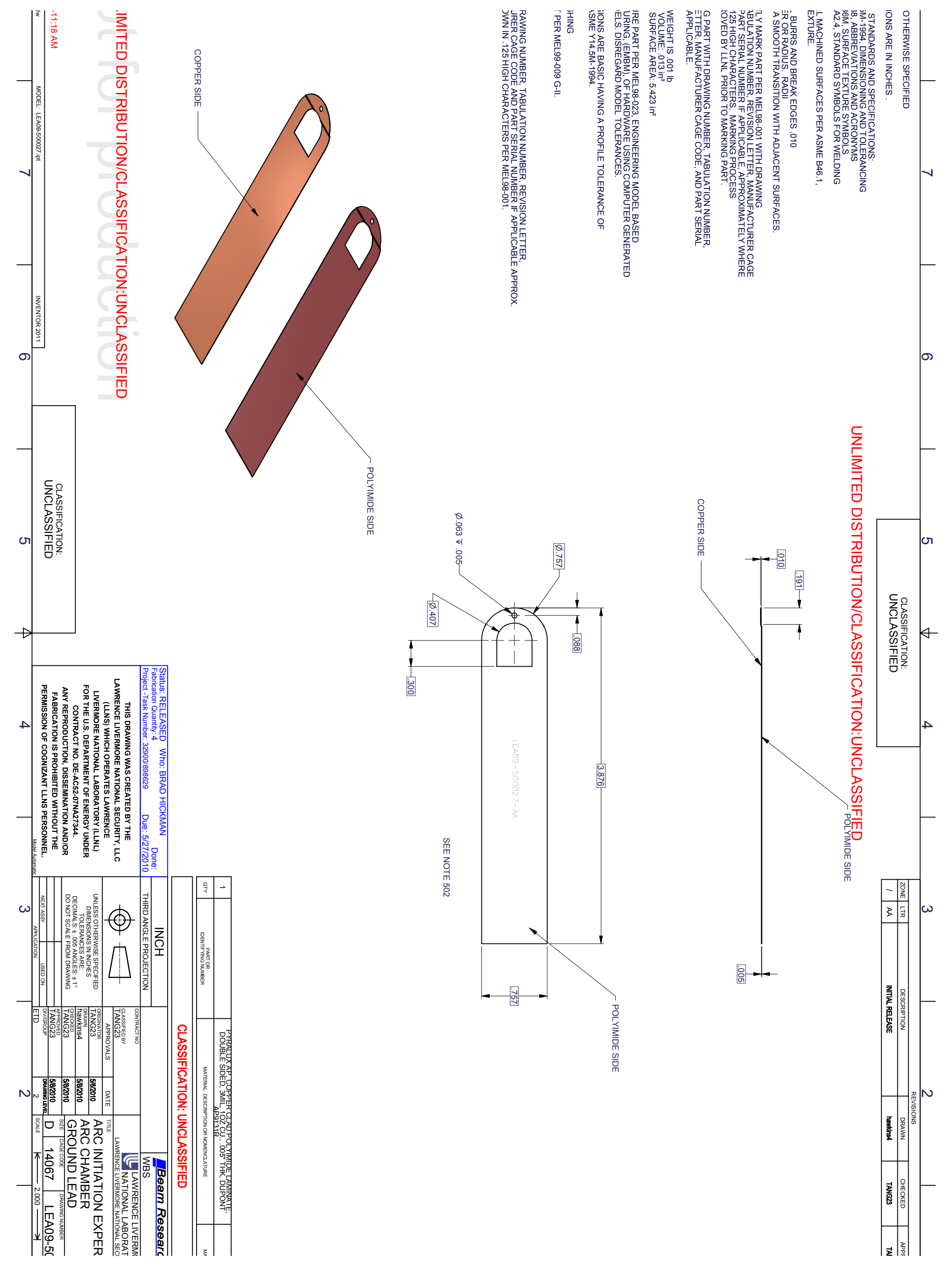




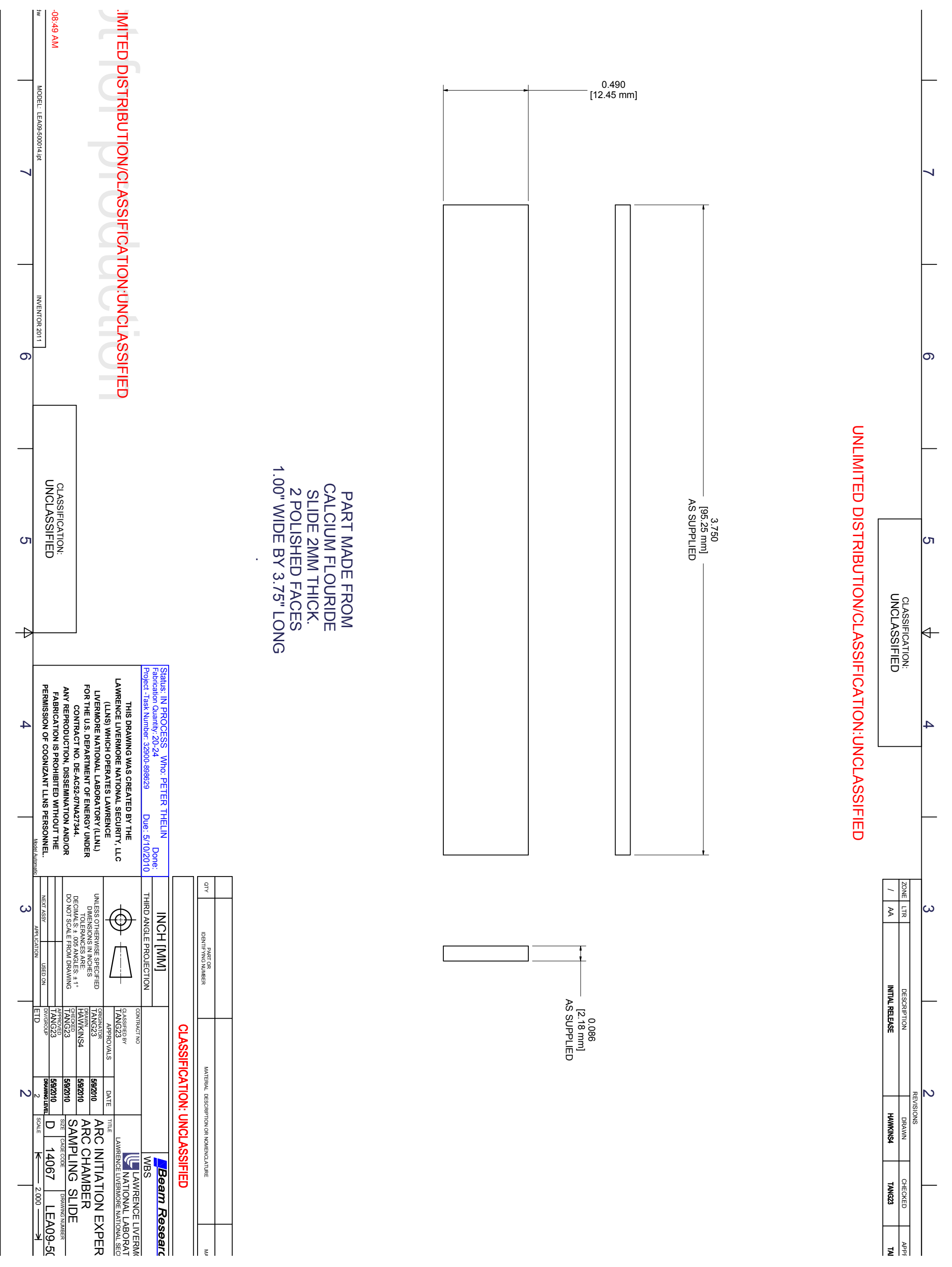



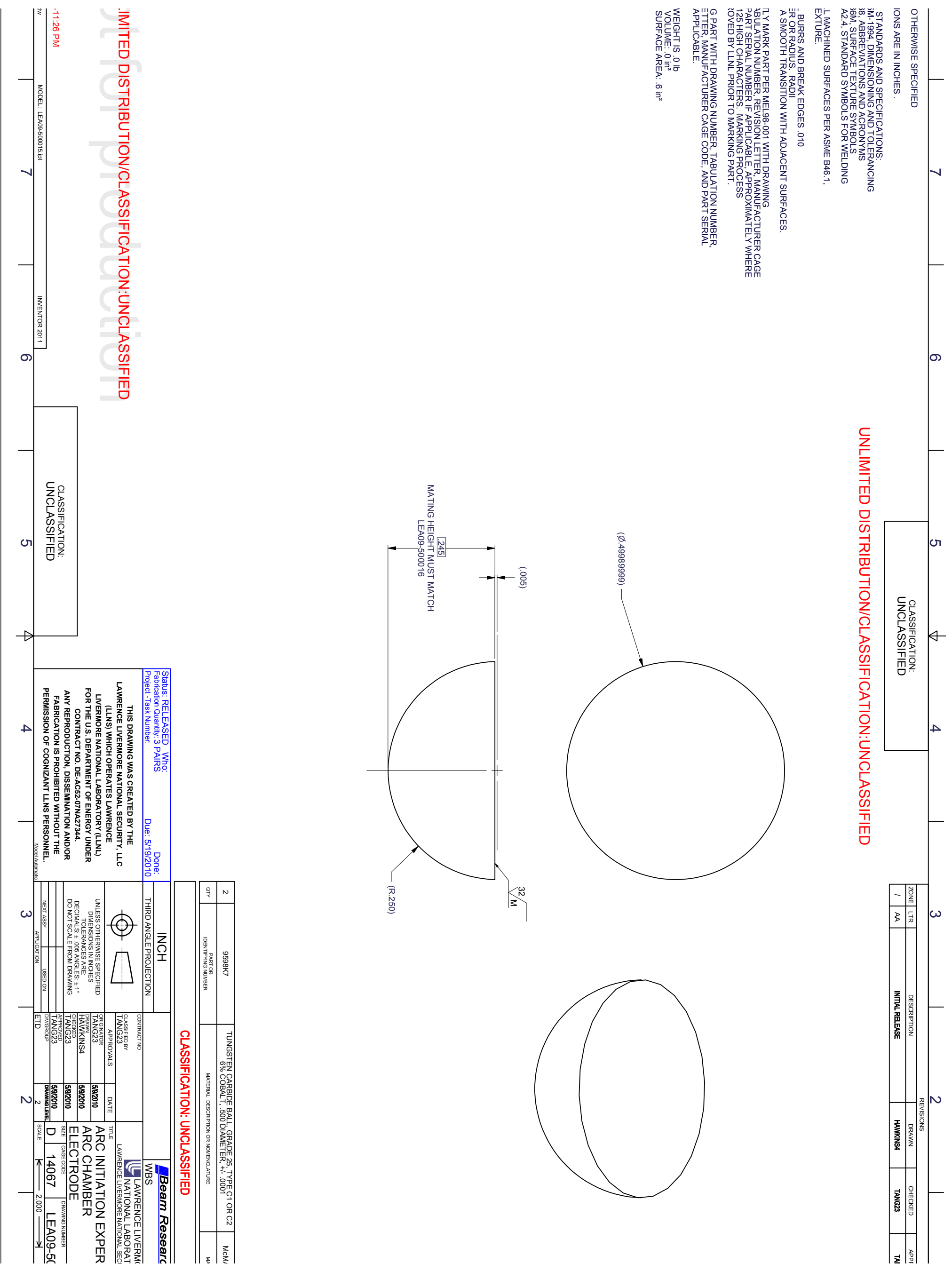


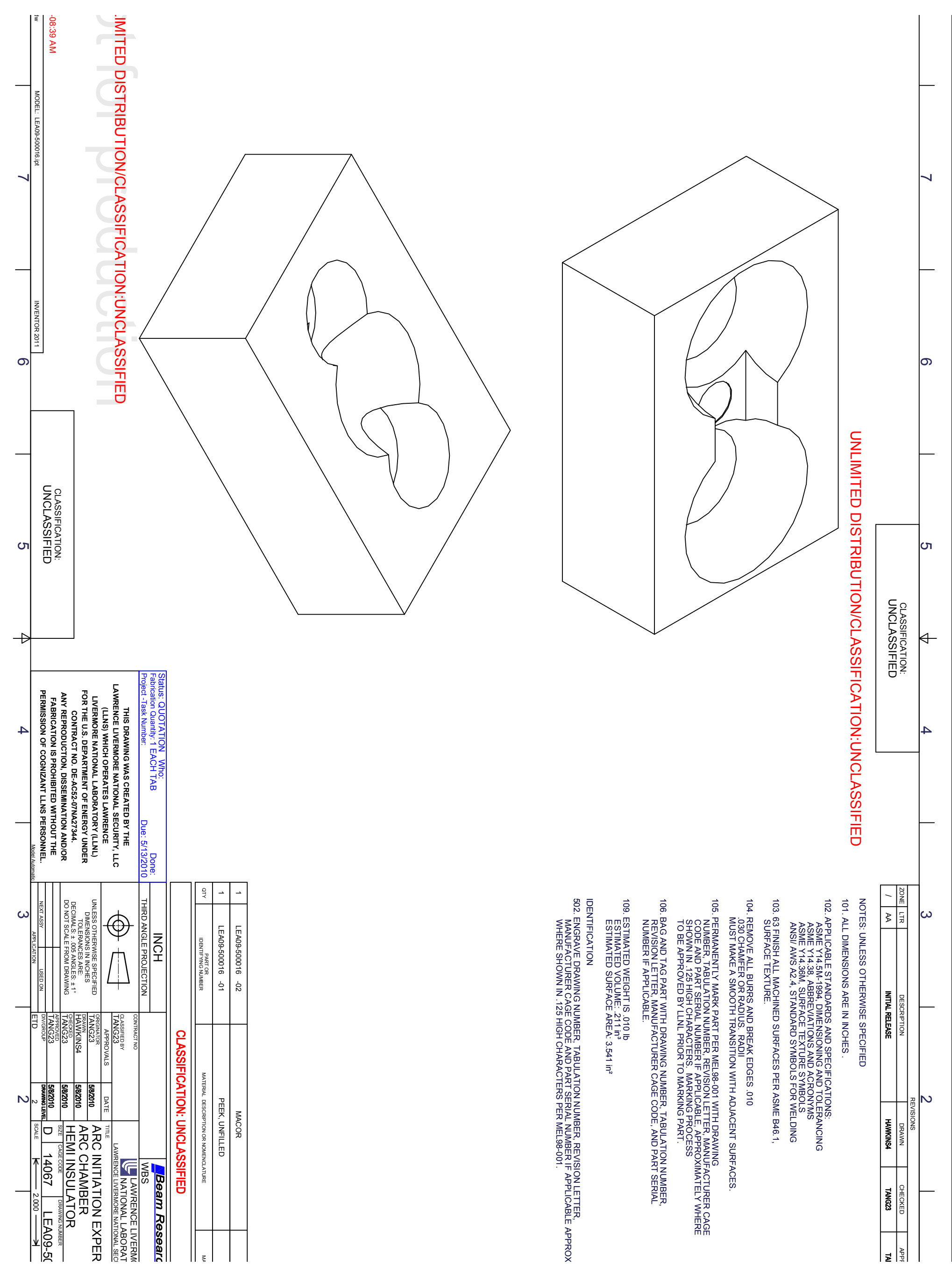




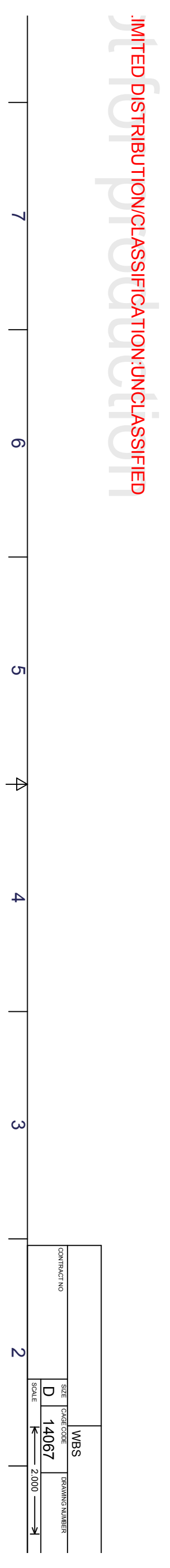

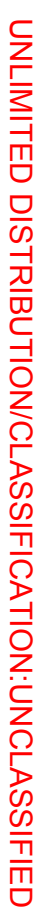




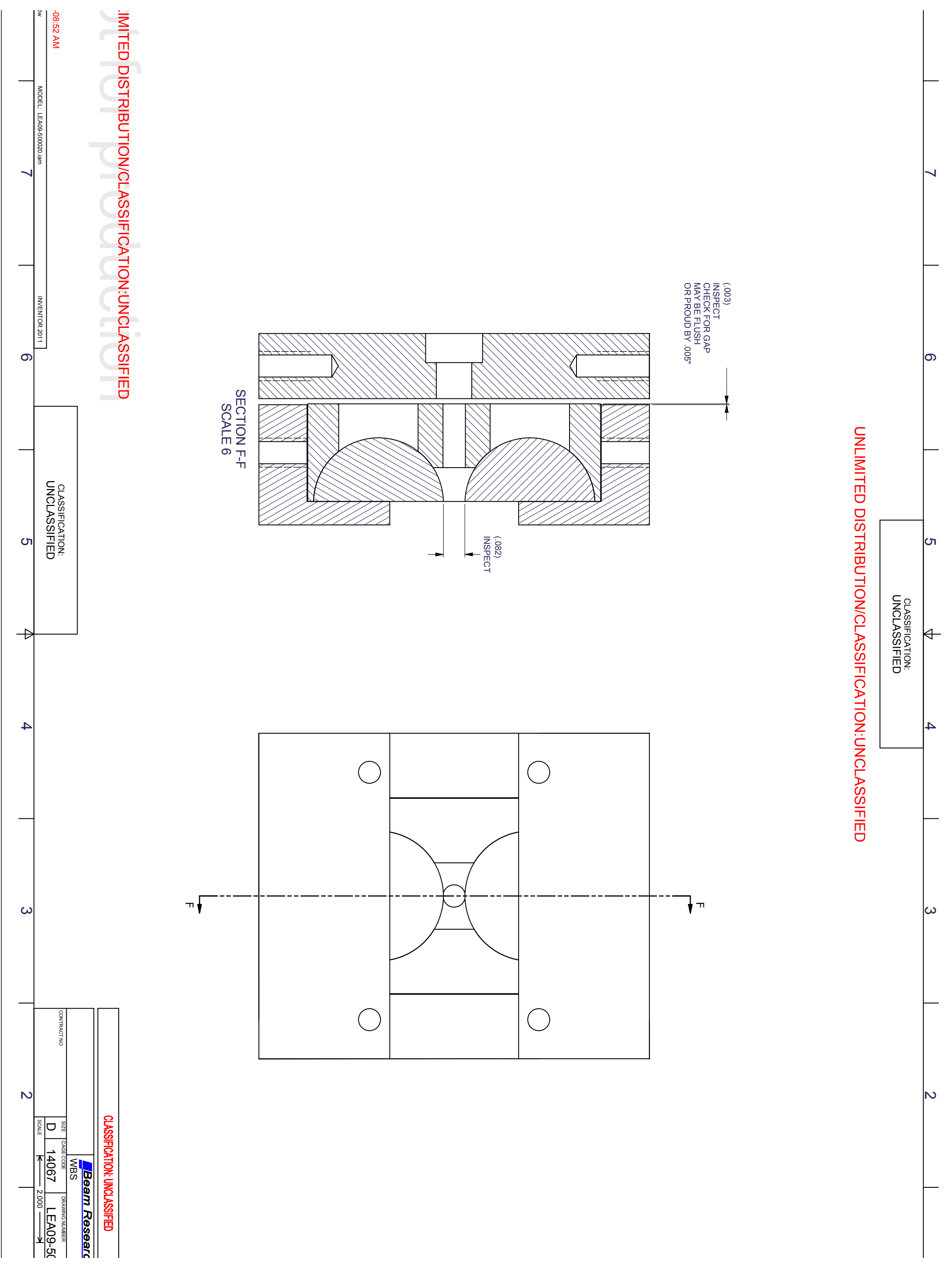




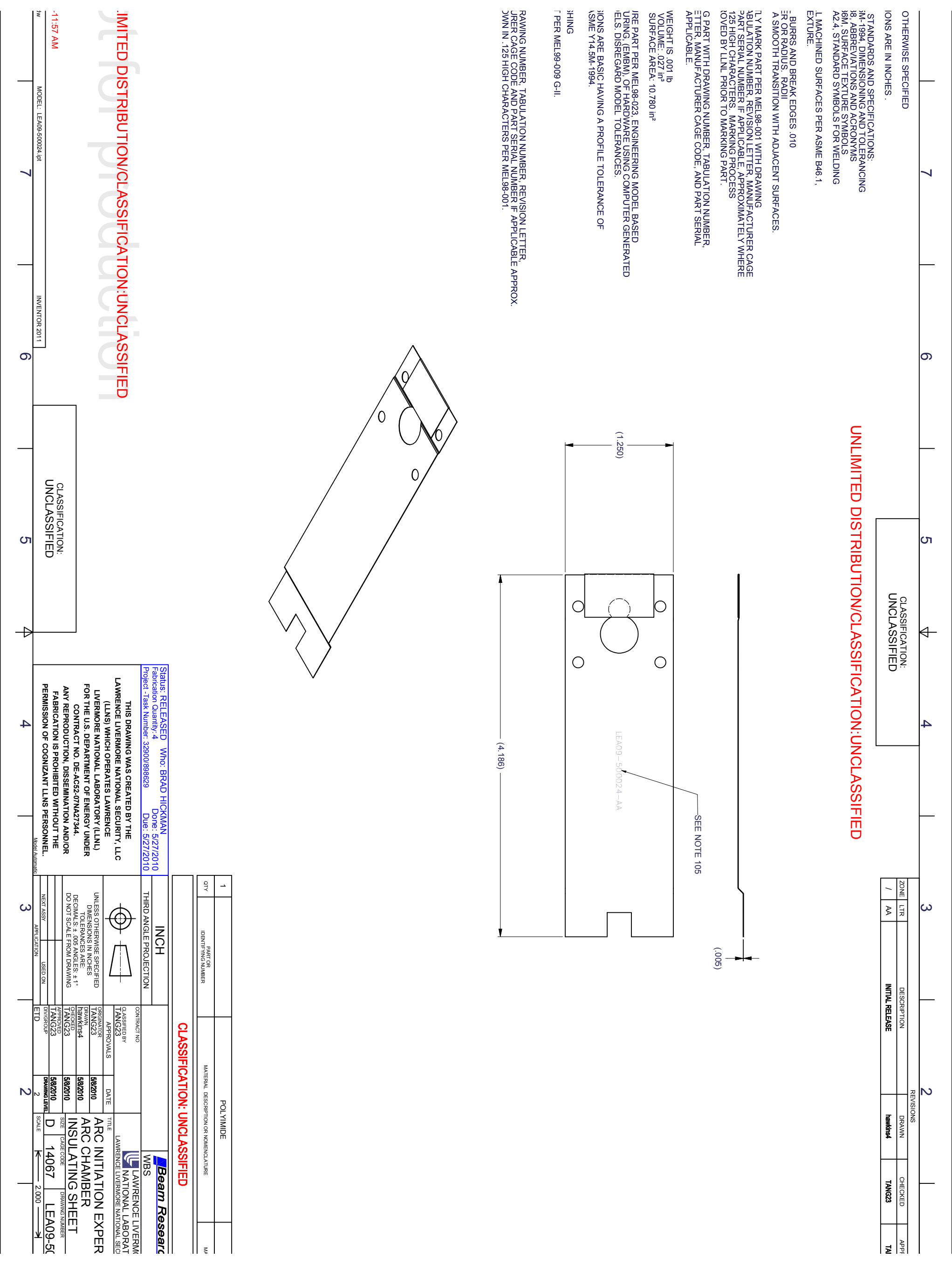



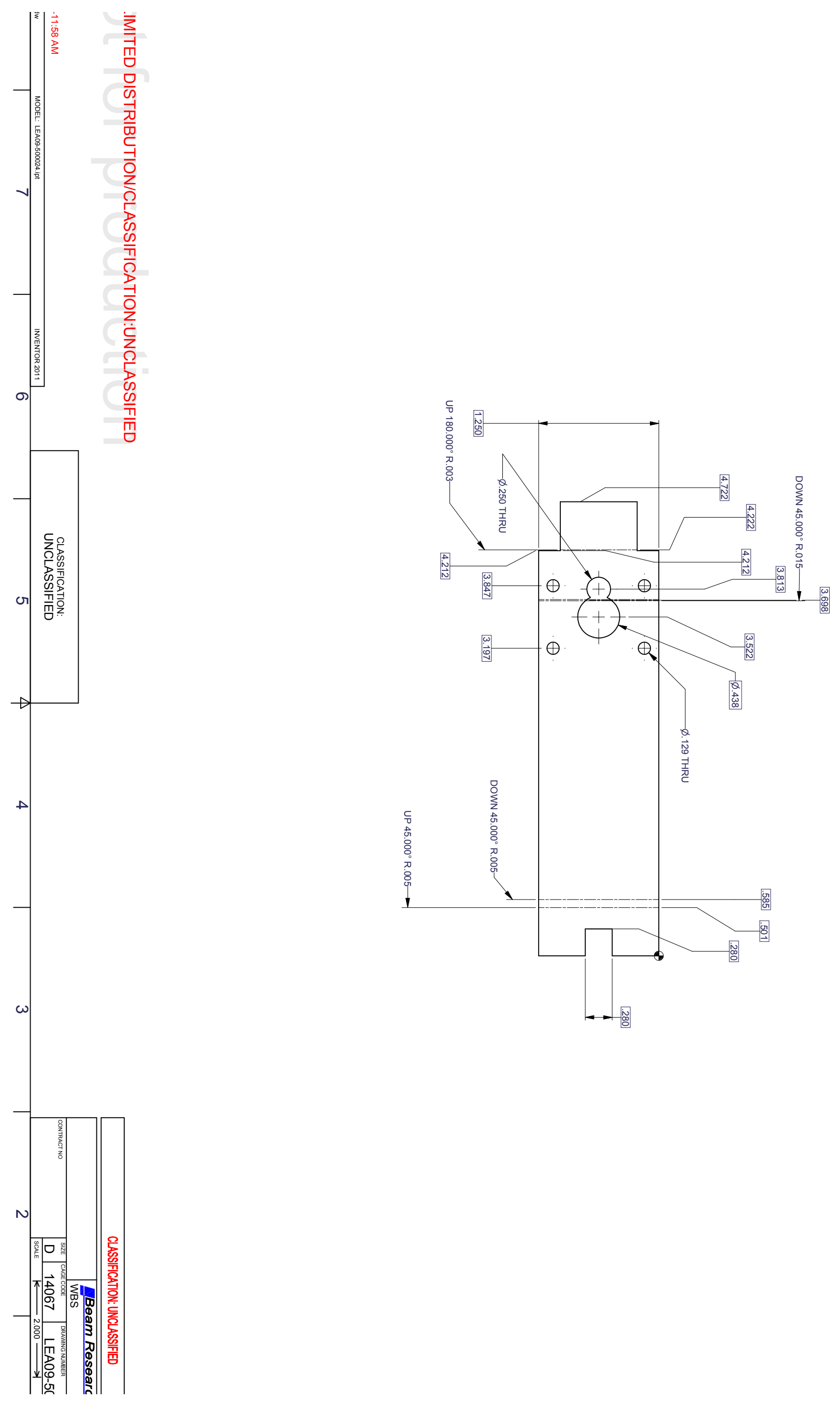

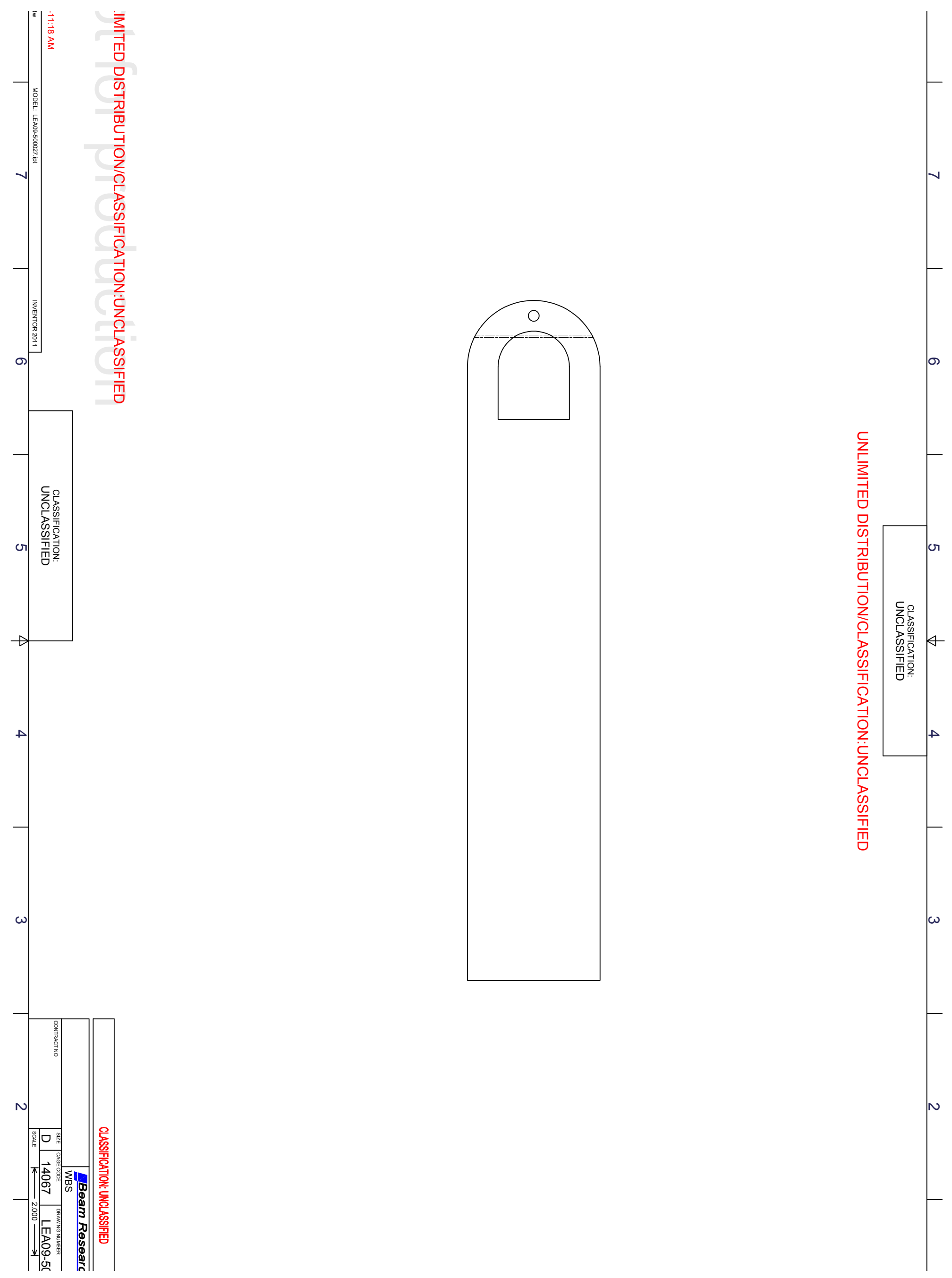


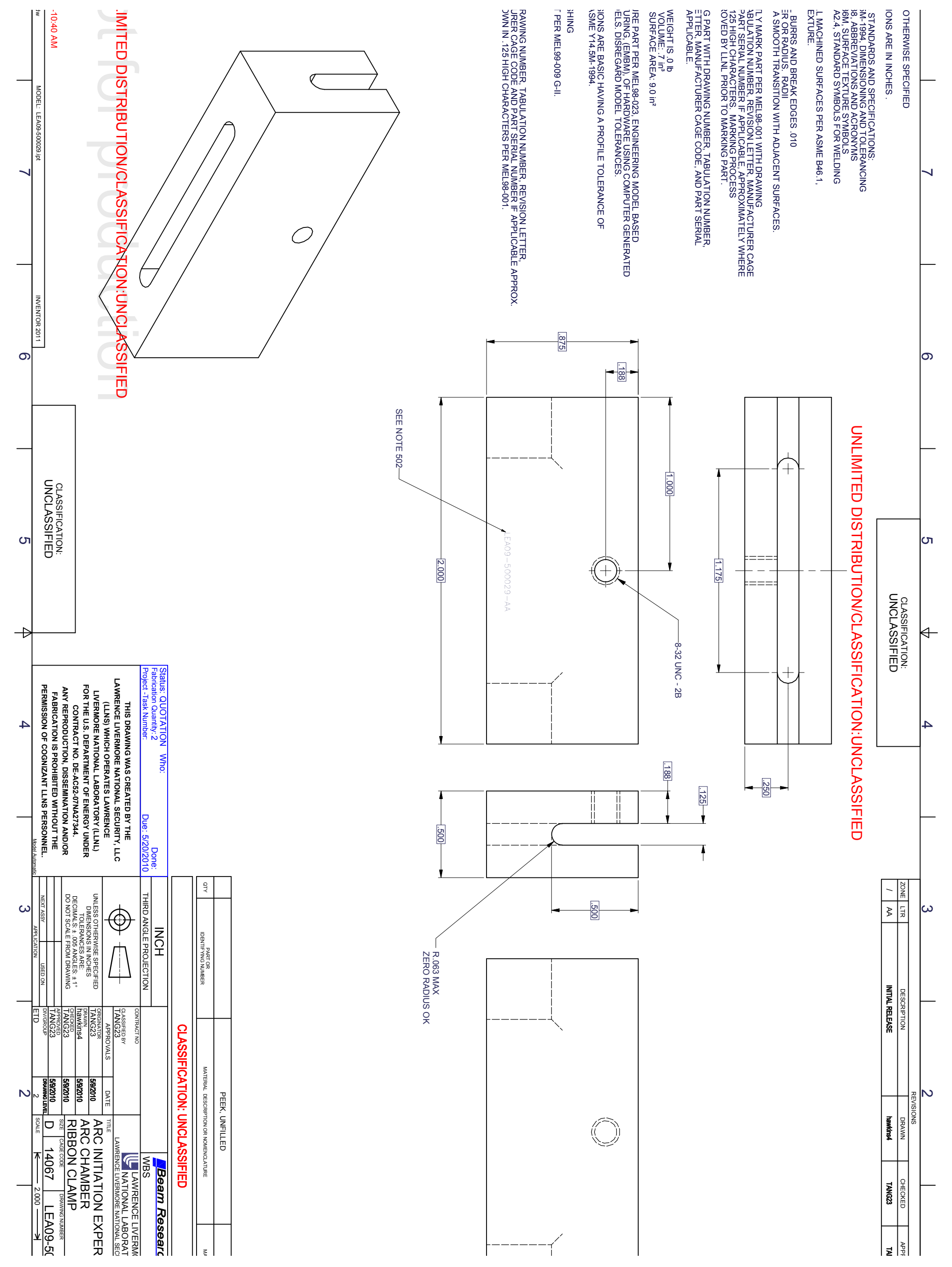




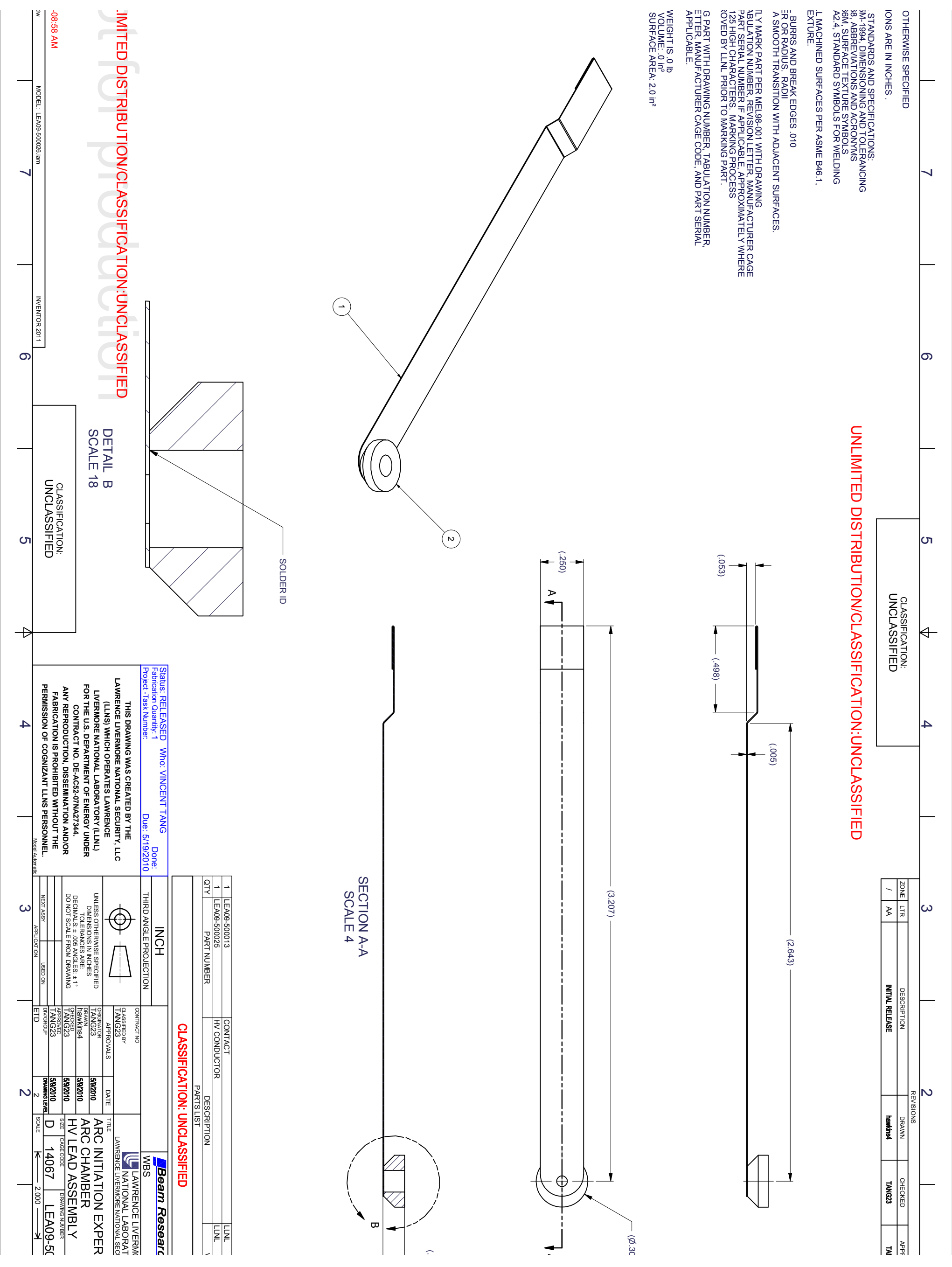




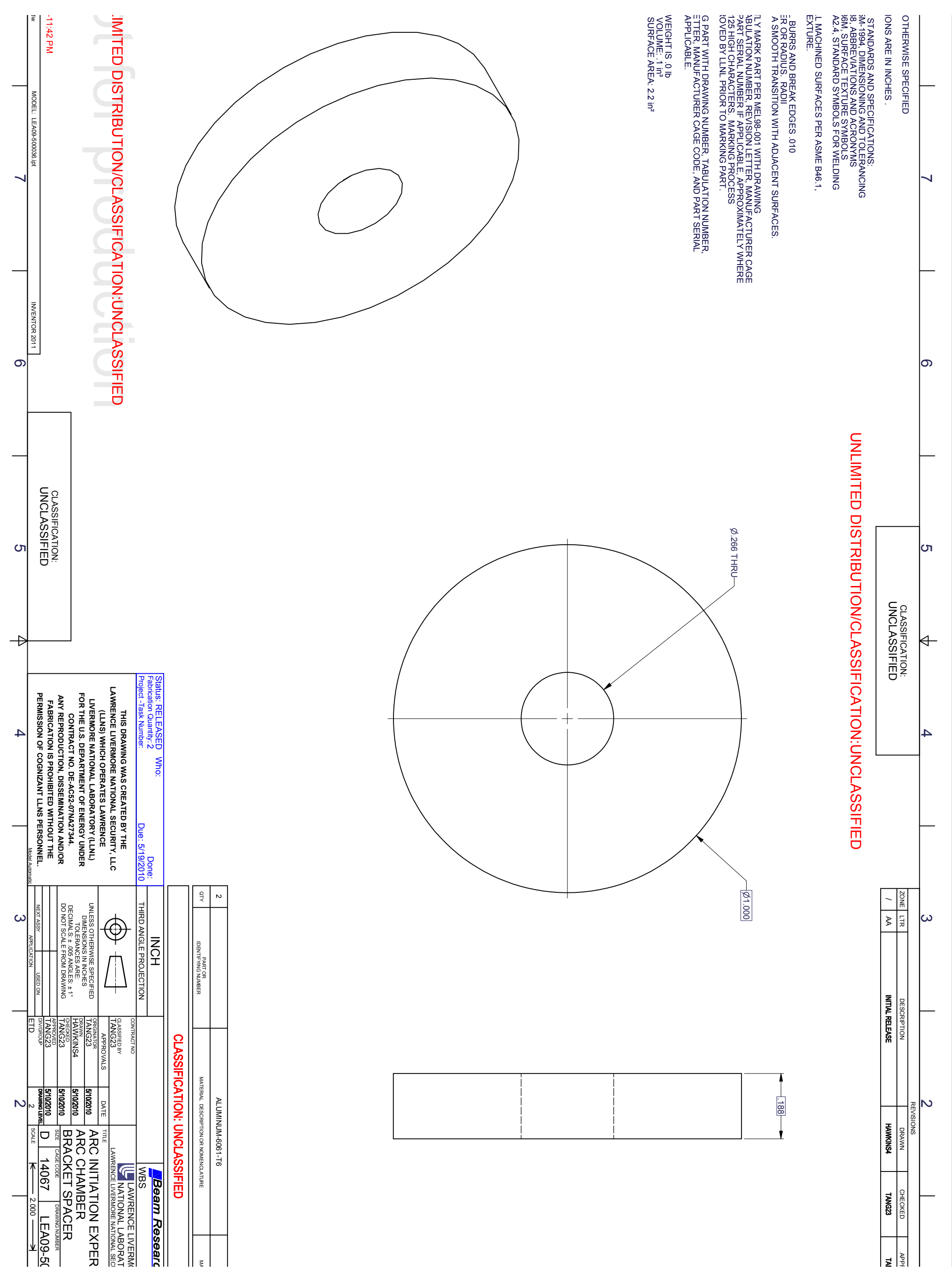



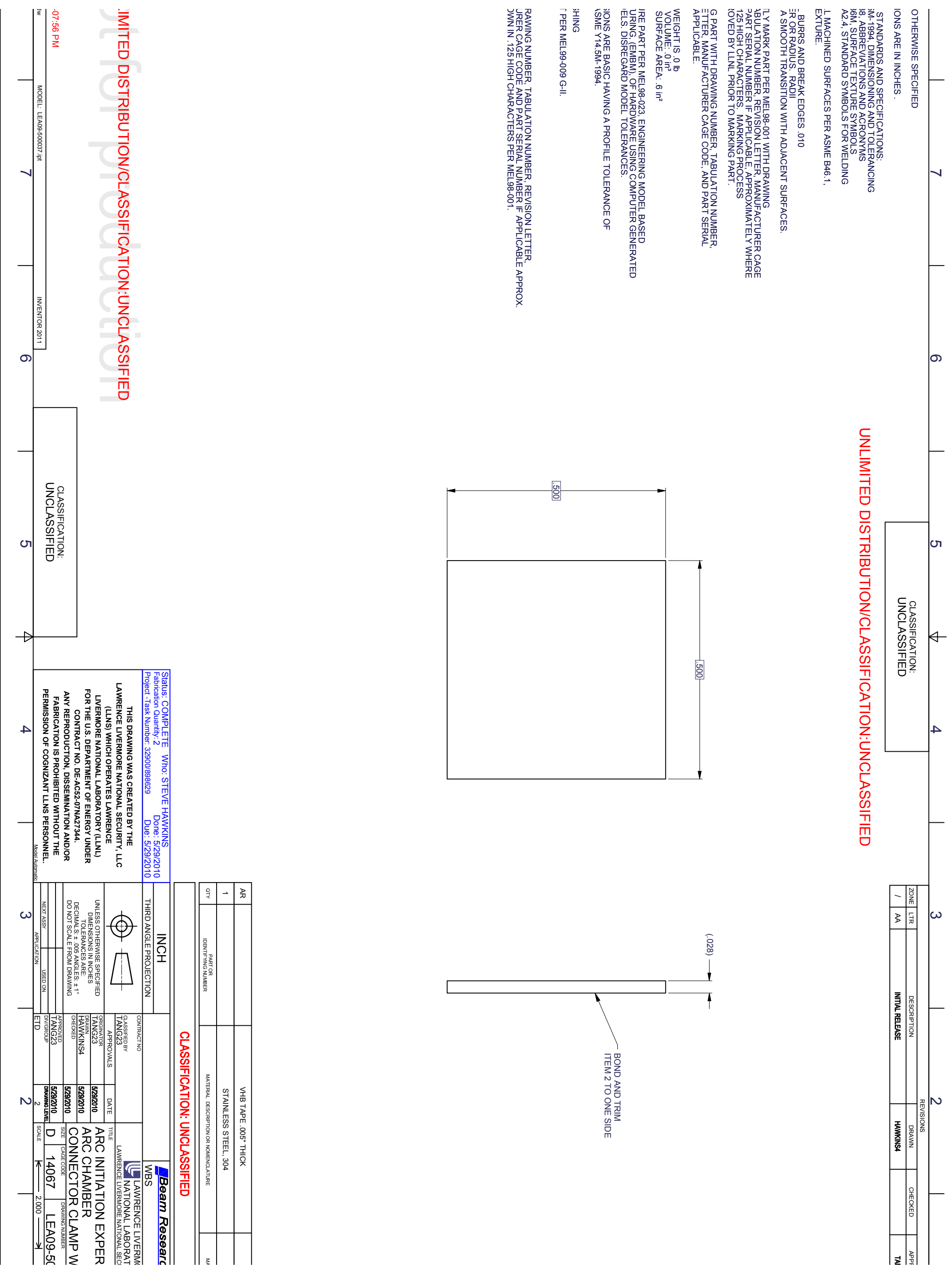


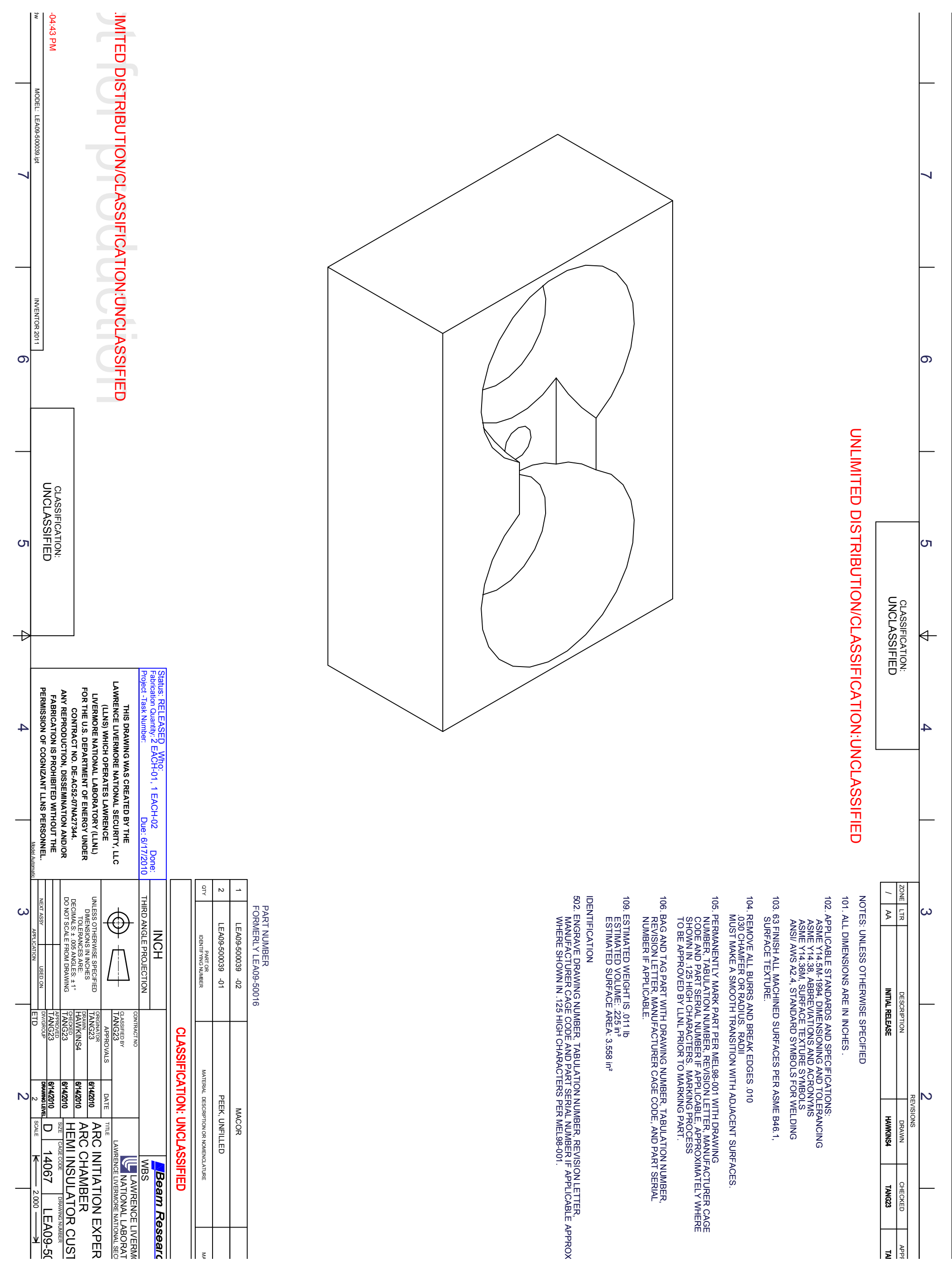




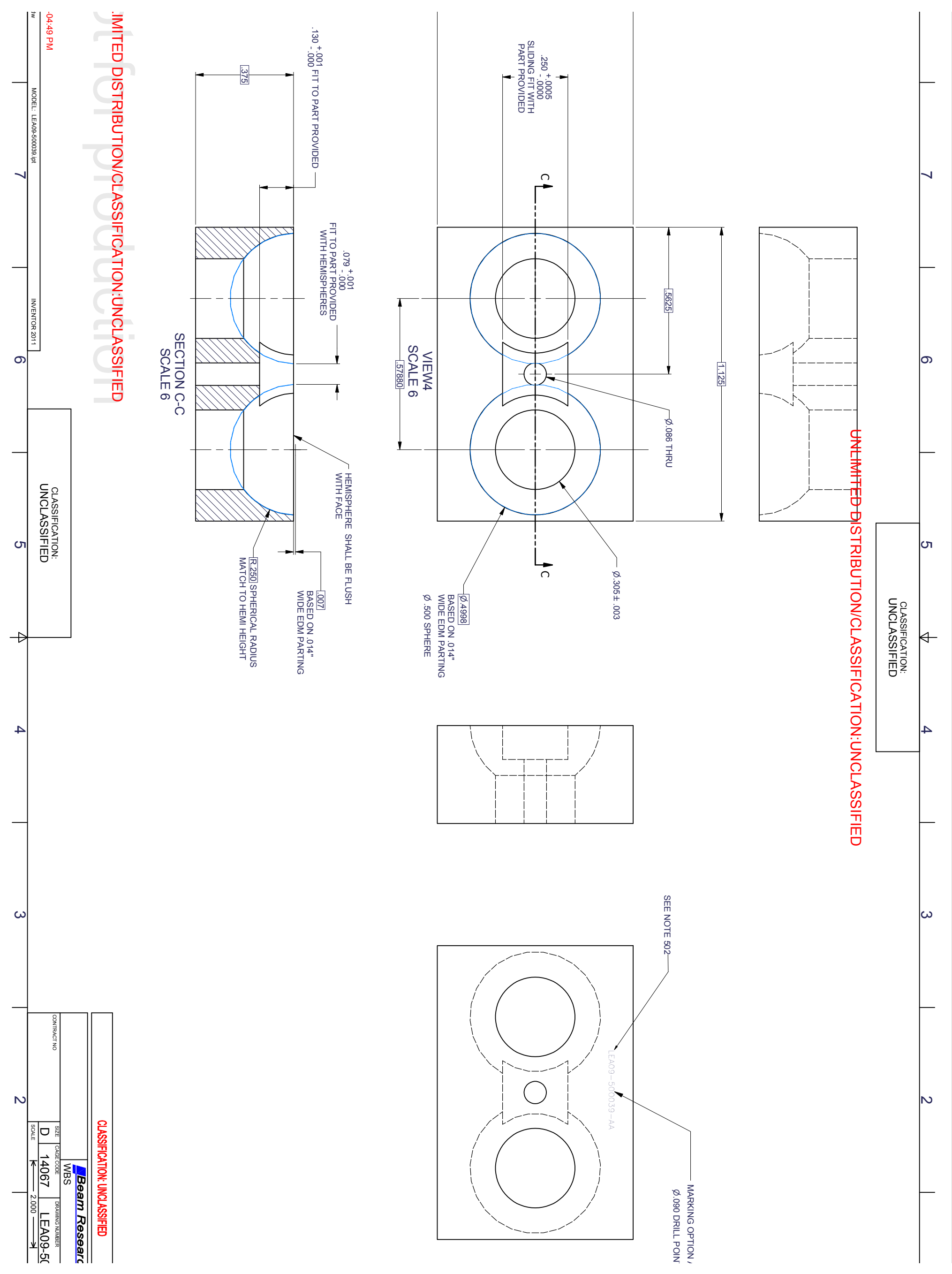




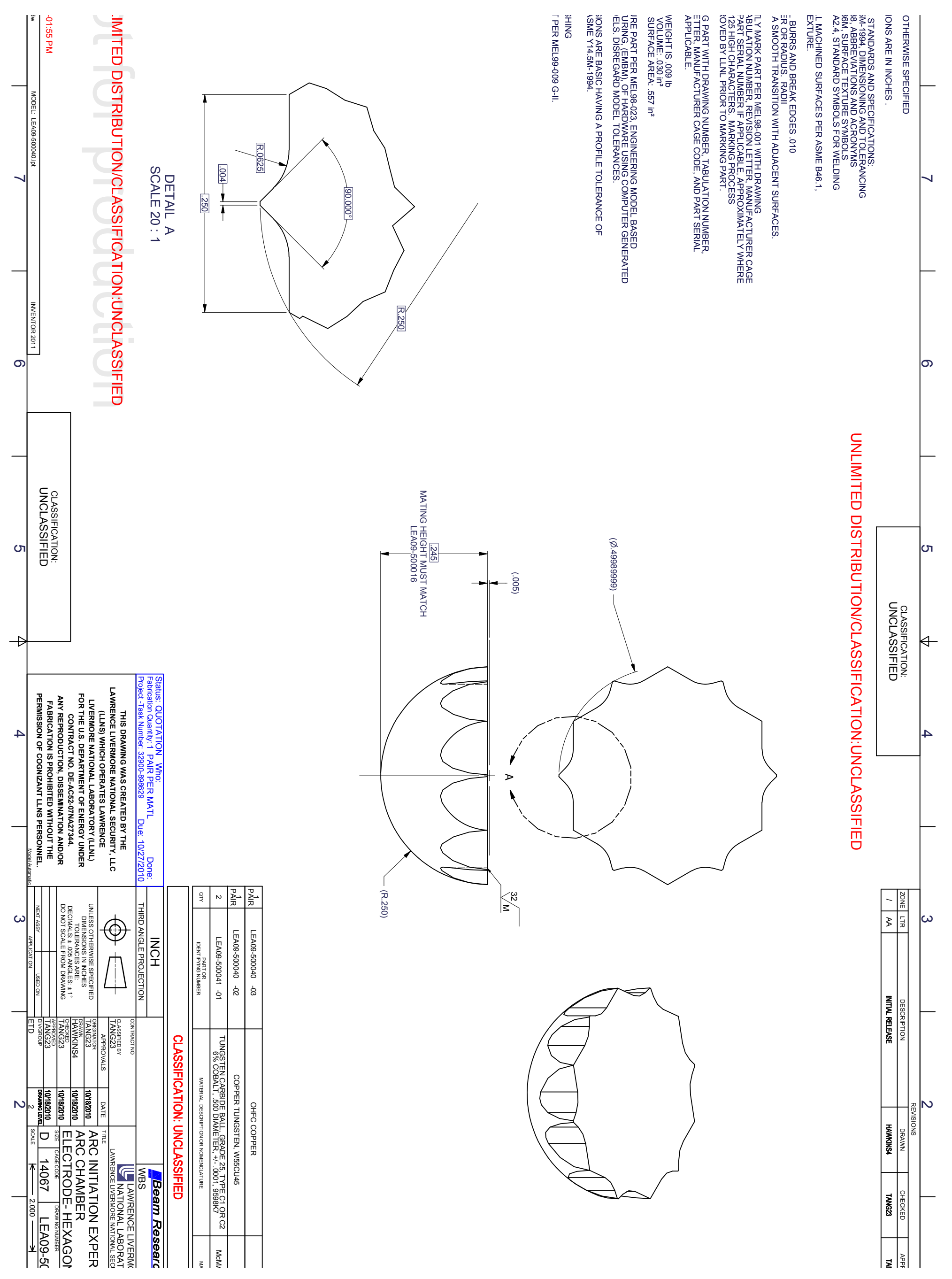




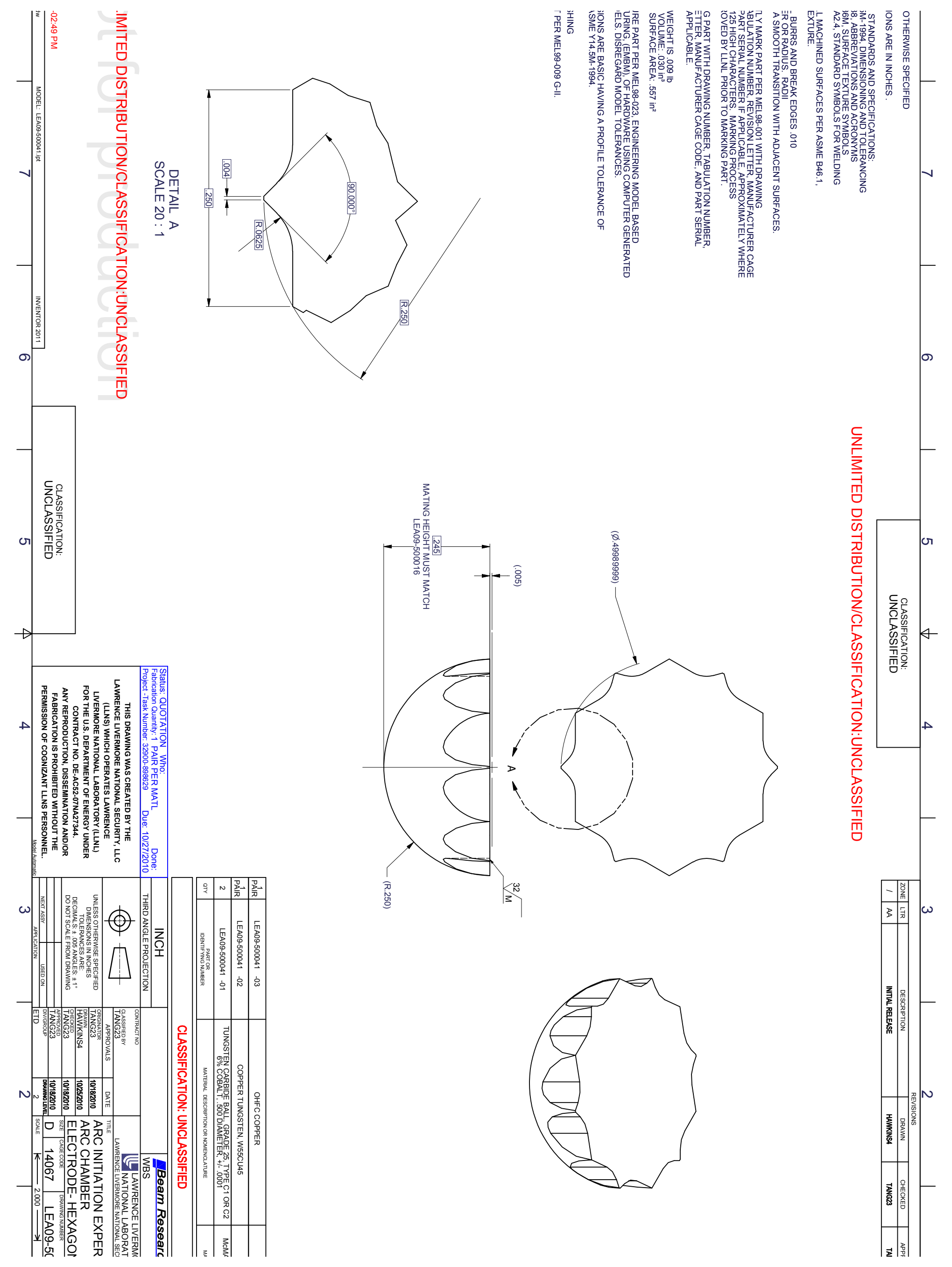


